\title{
Software Controlled Beam Steerable Antenna
}

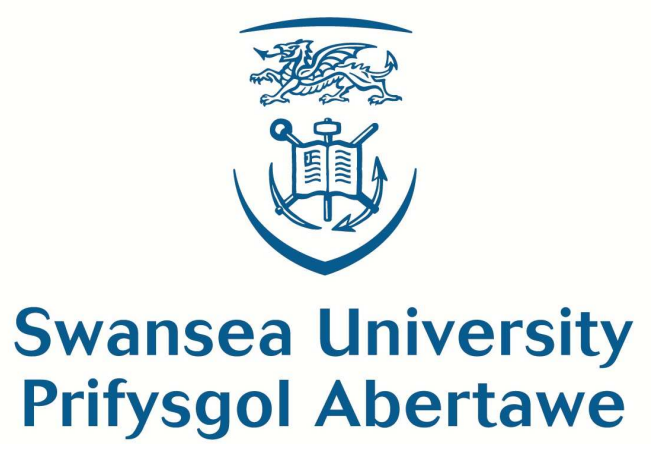

\section{Chandana Hasanga Goonesinghe}

College of Engineering

Swansea University

Submitted to Swansea University in fulfilment of the requirements for the

Degree of

Doctor of Philosophy

January 2018 

Dedicated to Amma, Thaththa, Charya and Heyan... 



\section{DECLARATION}

This work has not previously been accepted in substance for any degree and is not being concurrently submitted in candidature for any degree.

Signed (candidate)

Date

\section{STATEMENT 1}

This thesis is the result of my own investigations, except where otherwise stated. Where correction services have been used, the extent and nature of the correction is clearly marked in a footnote(s).

Other sources are acknowledged by footnotes giving explicit references. A bibliography is appended.

Signed (candidate)

Date

\section{STATEMENT 2}

I hereby give consent for my thesis, if accepted, to be available for photocopying and for inter-library loan, and for the title and summary to be made available to outside organisations.

Signed (candidate)

Date 



\section{Acknowledgements}

I am grateful to my PhD supervisor, Prof. Amit Mehta for all the support and guidance he has given me throughout my $\mathrm{PhD}$. He has continuously funded my $\mathrm{PhD}$ since 2013, ensuring that I was free to concentrate on my research fully, without any financial worries. His immense support, guidance and encouragement were the reasons I managed to complete my research and thesis on time.

I wish to thank my supervisor and friend, Dr. Arpan Pal, who has always been on hand to assist me during my research work in the lab. His support and assistance in completing my publications needs special mention.

My parents have always been pillars of strength in my life. Their words of encouragement went a long way to ensure I remained focused during my studies overseas. My loving wife Charya, has always stood by me through thick and thin. Last year, she gifted me with a beautiful son whose innocent smile reminds me every day how lucky I am.

I wish to thank Mr. Gareth Evans and Mr. David Moody from the College of Engineering for their assistance in fabricating my helmet antenna and providing other technical assistance during my research project. I also wish to thank my friend Dr. Gareth Ayres from LIS, for his assistance in the computer networking aspect of my research.

I am thankful to Prof. Dariush Mirshekar-Syahkal from Essex University and Prof. Hisamatsu Nakano from Hosei University, Japan for their comments in improving my published work.

I also wish to thank Mr. Nathan Clow from DSTL for continuing to fund my research project.

My friend Dr. Laurie Hughes and Mrs. Beth Hughes have kindly supported me on numerous occasions throughout my stay in Swansea over the years. Last, but not least, special mention goes out to Mr. Kapila Perera from London, who has been a father-like figure to me since the day I arrived in UK. 



\begin{abstract}
In this thesis I have presented a software controlled conformal beam steerable antenna for body wearable applications. The antenna is a square loop antenna conformal to a hemispherical shell. It generates 4 identical tilted beam patterns with high Directivity $(9.2$ $\mathrm{dBi}$ ). It has $4 \mathrm{RF}$ ports which are connected to a RF switch which conducts the beam switching based on the electronic voltages it receives from a control circuit. By switching one port at a time, while keeping the remaining 3 ports open, it is able to steer the beam covering the full $360^{\circ}$ azimuth range. An intelligent 'scan, analyse, lock and monitor' algorithm has been used to lock the antenna beam to the direction of the strongest signal. This algorithm runs on a portable hardware platform connected to the RF switch.

The thesis findings cover a study on the conformal nature of this square loop antenna on a variety of doubly curved surfaces. Its limits on bending are reported. It then reports the results of two experimental studies conducted to test the robustness of the beam switching algorithm. The first experiment involves using the antenna as a Base Station antenna which directs its beam towards a roaming mobile user based on the feedback it receives form the user. The second experiment involves using the antenna as a mobile user while the Base Station antenna is fixed. The algorithm switches ON the antenna's 4 ports, one at a time, and then locks it to the port with the highest received signal strength. It was experimentally proven that in a weak EM environment where the received signal strength is close to the noise floor, the antenna running the beam switching algorithm was able to maintain high throughput in a HD video call conferencing application while conventional methods failed.
\end{abstract}





\section{Table of contents}

$\begin{array}{ll}\text { List of figures } & \text { xv }\end{array}$

List of tables $\quad$ xix

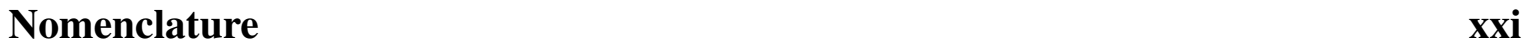

1 Introduction 1

1.1 Statement of topic and focus . . . . . . . . . . . . . . . 1

1.1.1 Vision and motivation for the research . . . . . . . . . . 1

1.1.2 How we can locate it within broader work? . . . . . . . . . 3

1.1.3 Choice of research setting and data collection strategy . . . . . . 4

1.2 Outline of this thesis $\ldots \ldots \ldots \ldots$

2 Background $\quad 11$

2.1 Introduction . . . . . . . . . . . . . . . 11

2.2 Multipath propagation . . . . . . . . . . . . . 11

2.2.1 Problems caused by multipath . . . . . . . . . . . . . 12

2.2.2 Multipath mitigation . . . . . . . . . . . . 15

2.3 Military communication in multipath environments . . . . . . . . . 22

2.3 .1 Jamming Avoidance . . . . . . . . . . . . . . . . . 23

2.3.2 Improving range and battery life . . . . . . . . . . . 23

2.4 Problem statement $\ldots \ldots \ldots \ldots \ldots . \ldots \ldots$

2.5 Proposed solution . . . . . . . . . . . . . . . . . . . 24

3 Literature review $\quad 27$

3.1 Introduction . . . . . . . . . . . . . . . . 27

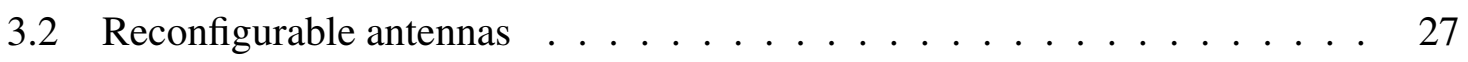

$3.2 .1 \quad$ A historical perspective . . . . . . . . . . . . . 28

3.3 Pattern reconfigurable antennas using Diodes and switches . . . . . . . . 31 
3.4 Pattern reconfigurable antennas using Frequency Selective Surfaces _ . . . 46

3.5 Pattern reconfigurable antennas using High Impedance Surfaces . . . . . . 50

3.6 Pattern reconfigurable antennas using non-planar structures . . . . . . . . . 51

3.7 Pattern reconfigurable square loop antenna . . . . . . . . . . . . . . 53

3.8 Conformal pattern reconfigurable antennas . . . . . . . . . . . . . . . 61

3.9 Plasma reconfigurable antennas . . . . . . . . . . . . . . . . . . . 64

3.10 Conformal helmet antennas . . . . . . . . . . . . . . . . . 66

3.11 Beam switching algorithms . . . . . . . . . . . . . . . . 68

3.12 Conclusion . . . . . . . . . . . . . . 70

4 Conformal $2.4 \mathrm{GHz}$ beam steering antenna for high throughput applications in weak EM environments $\quad 73$

4.1 Introduction . . . . . . . . . . . . . . . . . . . 73

4.2 Background . . . . . . . . . . . . . . . . . 74

4.3 System design . . . . . . . . . . . . . . . . . . . 74

4.3.1 Conformal beam steerable antenna on a hemisphere . . . . . . . . . 74

4.3.2 Integration of conformal antenna with $2.4 \mathrm{GHz}$ WiFi router . . . . 76

4.4 Antenna configuration and results . . . . . . . . . . . 76

4.4.1 Hemispherical square loop antenna (HSLA) . . . . . . . . . . 76

4.4.2 Simulated and experimental results . . . . . . . . . . . . 79

4.5 Parameter study . . . . . . . . . . . . . . . . . . 79

4.5.1 Effects of variation of separation between ground plane and radiating strips on the radiation pattern . . . . . . . . . . . . . 79

4.5.2 Effects of substrate width on radiation pattern . . . . . . . . 86

4.5.3 Effects of substrate bending on radiation pattern . . . . . . . . . 86

4.6 Integration of HSLA with $2.4 \mathrm{GHz}$ WiFi system . . . . . . . . . . . . 88

4.6.1 Experimental setup . . . . . . . . . . . . 88

4.7 Conclusion . . . . . . . . . . . . . . . . . 93

5 HSLA Integrated with Raspberry Pi for a mobile user using high throughput applications $\quad 95$

5.1 Introduction . . . . . . . . . . . . . . . . . . . 95

5.2 Background ........................... 96

5.3 Hemispherical Square Loop Antenna (HSLA) . . . . . . . . . . . . . . 97

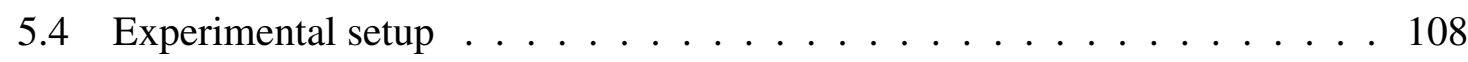

5.5 Throughput analysis . . . . . . . . . . . . . . . . . 108

5.6 Conclusion . . . . . . . . . . . . . . . . . 113 
6 Future Work $\quad 115$

6.1 Introduction . . . . . . . . . . . . . . . . . 115

6.2 Planar SLA for Ku band . . . . . . . . . . . . . . . . 116

6.3 Planar SLA with HHIS for K band . . . . . . . . . . . . . . . . . . 118

6.4 Planar SLA for Ka band . . . . . . . . . . . . . . . . . . . . . 121

6.5 DoA estimation using 4 beam SLA . . . . . . . . . . . . . . . 123

6.6 SSC and SEC schemes using SNR for HSLA . . . . . . . . . . . . 125

6.7 Implementation of an adaptive optimal switching threshold for HSLA . . . 126

6.8 Conclusion . . . . . . . . . . . . . . . 126

7 Conclusion $\quad 129$

7.1 Introduction . . . . . . . . . . . . . . . . . . . . . 129

7.2 Thesis summary . . . . . . . . . . . . . . . . 130

7.3 Contribution to knowledge . . . . . . . . . . . . . . . . . 134

$\begin{array}{ll}\text { References } & 137\end{array}$

$\begin{array}{lll}\text { Appendix A C code use for beam steering algorithm } & 153\end{array}$ 



\section{List of figures}

2.1 Multipath LOS and NLOS signals and the resultant signal at the receiver . . 13

2.2 Inter-Symbol interference caused by signal smearing die to multipath . . . . 14

2.3 Partial Cancellation due to Multipath . . . . . . . . . . . . . . . . . . 15

2.4 Polarization Ellipse obtained by plotting maximum of $\mathbf{E}$ over time . . . . 19

2.5 A 4-element linear antenna array with isotropic radiators . . . . . . . . . 21

3.1 Reconfigurable antenna using a semiconductor illuminated by a light source as presented in Ding et al. (1995) . . . . . . . . . . . . . . . . . 29

3.2 4-segment monopole antenna using optoelectronic switches, as presented in Freeman et al. (1992) . . . . . . . . . . . . . . . . . . . . 29

3.3 Pattern reconfigurable cubic antenna as presented in Sarrazin et al. (2009) . 31

3.4 U-slot reconfigurabe patch antenna by Qin et al. (2012) and simulated 3D Directivity pattern for configuration-I. . . . . . . . . . . . . . 33

3.5 Pattern reconfigurable antenna design and farfield Directivity pattern by Li et al. (2011b). . . . . . . . . . . . . . . . . . . . 35

3.6 Pattern reconfigurable antenna design by Nair and Ammann (2010). . . . . 36

3.7 Reconfigurable UWB notch antenna design by Chen et al. (2012). Top view (Left) and bottom view showing ground plane (Right). . . . . . . . . . . 38

3.8 Top view of 4-feed reconfigurable 4-patch antenna (Left) by Cao et al. (2012) and its feeding network incorporating RF MEMS switches (Right). . . . . .

3.10 Pattern reconfigurable antenna design with alternate reflector and radiator sides by Zhang et al. (2012). . . . . . . . . . . . . . . . . . . . . . . 43

3.11 Conformal pattern reconfigurable spiral antenna by Gong et al. (2013). . . . 43

3.12 The frequency selective reconfigurable antenna structure by Zhang et al. (2013). 45

3.13 Unit cell containing two reflectors with varactor diodes as shown in Zhang et al. (2013). . . . . . . . . . . . . . . . . . . 46

3.14 Top view (Left) and bottom view (Right) of the unit cell design of FSS proposed by Yuan et al. (2013). . . . . . . . . . . . . . . . . . . . . . 47 
3.15 Reconfigurable antenna design using FSS by Edalati and Denidni (2013). Clockwise from top Left, Side view, Top view, 3D view and the dipole at the center. . . . . . . . . . . . . . . .

3.16 Design of pattern reconfigurable planar spiral antenna over a HIS structure by Deo et al. (2009b). . . . . . . . . . . . . . . . . 50 50

3.17 Graphene based reconfigurable HIS block suggested by Huang et al. (2012). 51

3.18 Design of pattern reconfigurable antenna using switched printed elements by Shi et al. (2012) . . . . . . . . . . . . . . . . . . . 52

3.19 Design of heptaband reconfigurable loop antenna by Li et al. (2011a). (Top) the loop antenna dimensions before folding. (Bottom) the folded loop antenna integrated with feedline. . . . . . . . . . . . . . . . 54

3.20 The design of SLA by Mehta and Mirshekar-Syahkal (2007). . . . . . . . . 55

3.21 The SLA with short circuited feed terminations design (Left) and the simulated farfield Gain pattern (Right) by Pal et al. (2008). . . . . . . . . . . . 56

3.22 Feeding mechanisms for the 4 port SLA proposed by Pal et al. (2013a) and Deo et al. (2011a) . . . . . . . . . . . . . . . . . . 57

3.23 The SLA on a hybrid high impedance surface (HHIS) by Deo et al. (2010). 59

3.24 The dual band capacitively coupled SLA design by Pal et al. (2013b). . . . 60

3.25 The frequency response of the return loss coefficient of the DBCCSLA by Pal et al. (2013b). . . . . . . . . . . . . . . . . 6 60

3.26 Body wearable reconfigurable antenna by Javed et al. (2015). . . . . . . . . 62

3.27 Flexible body wearable reconfigurable antenna design (Left) by Ha and Jung (2011) and the prototype (Right) . . . . . . . . . . . . . . 63

3.28 Reconfigurable dipole plasma antenna design (Left) by Melazzi et al. (2017) and the prototype (Right). . . . . . . . . . . . . . . 65

3.29 Reconfigurable monopole plasma antenna array prototype (Left) by Sun et al. (2016) and the simulated $1 \times 8$ array results (Right). . . . . . . . . . . 66

3.30 Inside helmet antenna by Wang (2006). . . . . . . . . . . . . . . . 67

3.31 Helmet patch antenna prototype by Herold et al. (2007). . . . . . . . . . . . 68

4.1 The conformal SLA on spherical surface and its integration with WiFi router system for throughput enhancement. . . . . . . . . . . . . 75

4.2 (a) Top (b) Perspective (c) Bottom and (d) Cross-section (X-Z plane) views of the Hemispherical SLA (HSLA). ( $t=4 \mathrm{~mm}, W_{a}=96 \mathrm{~mm}, W_{g}=162 \mathrm{~mm}$, $h_{1}=8.5 \mathrm{~mm}$ and $\varepsilon_{r 1}=3.4$ (Kevlar), $h_{2}=14 \mathrm{~mm}$ and $\varepsilon_{r 2}=1.04$ (Foam), $c=a=175 \mathrm{~mm}, \mathrm{r}=274.5 \mathrm{~mm}) . \ldots \ldots \ldots 77$

4.3 Fabricated HSLA on a hemispherical shell . . . . . . . . . . . 78 
4.4 Frequency response of reflection coefficient $\left(\left|S_{11}\right|\right)$ of HSLA and its input impedance . . . . . . . . . . . . . . . . . . 80

4.5 Fabricated and simulated models of component for impedance matching, its equivalent circuit and $S_{11}$ of HSLA after impedance matching $\ldots . . . .81$

4.6 Elevation and Azimuth plane polar plots of HSLA . . . . . . . . . . . . . 82

4.7 Demonstration of beam steering using HSLA . . . . . . . . . . . . 83

4.8 The change in radiation pattern and Gain when total profile height was varied 84

4.9 The change in radiation pattern and Gain of HSLA when curvilinear distance was varied . . . . . . . . . . . . . . . . . . . . 85

4.10 The effects of substrate bending on conformal SLA . . . . . . . . . . 87

4.11 The microcontroller circuit for controlling SP4T switch and the hardware setup for experiment. . . . . . . . . . . . . . . . . 89

4.12 The automatic beam switching algorithm flowchart . . . . . . . . . . 90

4.13 Screenshots of video call statistics when automatic beam switching was not used and when automatic beam switching was used. . . . . . . . . . . . 92

5.1 Top view and side view of the planar SLA on a circular substrate. . . . . . . 98

5.2 Top view and side view of SLA on a hemispherical substrate. . . . . . . . . 99

5.3 Effect of substrate bending on the radiation pattern at $2.45 \mathrm{GHz}$. . . . . . . 101

5.4 Reflection coefficient and (Inset) the input impedance of the HSLA. . . . . 102

5.5 The Microstripline component for matching the input impedance. . . . . . 103

5.6 The Polar Plots of the HSLA in the Azimuth plane and the Elevation plane for $2.35 \mathrm{GHz}, 2.45 \mathrm{GHz}$ and $2.6 \mathrm{GHz}$. . . . . . . . . . . . . 105

5.7 Schematic of the beam-steering system. . . . . . . . . . . . . . . 106

5.8 The experimental setup. . . . . . . . . . . . . . . . . . . . . 107

5.9 The enhanced beam switching algorithm. . . . . . . . . . . . . . . . 109

6.1 Design of $4.65 \lambda_{g}$ SLA. . . . . . . . . . . . . . . . . . 116

6.2 Simulated results of the $4.65 \lambda_{g}$ SLA designed for $12 \mathrm{GHz} . \quad \ldots \ldots . . . . \quad 117$

6.3 Design of scaled SLA with HHIS for $25 \mathrm{GHz}$. Perspective view (Top), Top view (Middle) and Side view showing the HHIS (Bottom). . . . . . . . . . 119

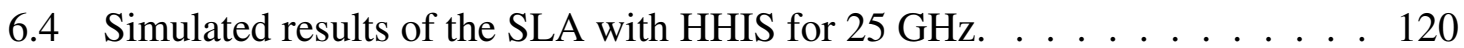

6.5 Perspective view of the designed SLA scaled for $30 \mathrm{GHz}$. . . . . . . . . . 121

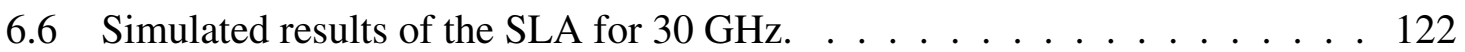

6.7 Illustration of beam switching sequence of HSLA for DoA estimation algorithm. . . . . . . . . . . . . . . . . . . . . 124 



\section{List of tables}

4.1 The variation of vertical axis $\boldsymbol{c}$ and horizontal axis $\boldsymbol{a}$ for the 7 shapes of the conformal SLA studied. . . . . . . . . . . . . . . 88

4.2 Link budget calculation for experimental setup . . . . . . . . . . . 91

4.3 RSSI readings for the four ports at each location during a live HD video call 91

5.1 Summary of throughput analysis for beam switching algorithm operation . 111 



\title{
Nomenclature
}

\author{
Acronyms / Abbreviations
}

AF Array Factor

AWGN Additive White Gaussian Noise

BER Bit Error Rate

BS Base Station

CP Circular Polarization

CPW Co-planar Waveguide

DLL Delay Locked Loop

DoA Direction of Arrival

DSP Digital Signal Processing

EBG Electromagnetic Band Gap

FE BST Ferroelectric Barium Strontium Titanate

FSS Frequency Selective Surface

GNSS Global Navigation Satellite System

GPS Global Positioning System

HD High Definition

HIS High Impedance Surface

HIS Hybrid Impedance Surface 
ISI Inter-Symbol Interference

LHCP Left Hand Circularly Polarized

LOS Line of Sight

MAP Maximum a posteriori

MEMS Microelectromechanical Systems

MLE Maximum Likelihood Estimator

MMSE Minimum Mean-Square Error

MS Mobile Subscriber

NLOS Non-Line of Sight

OFDM Orthogonal Frequency Division Multiple Access

PDF Probability Density Function

POCS Projection Onto Convex Sets

RHCP Right Hand Circularly Polarized

RSS Received Signal Strength

SC Selection Combining

SEC Switch and Examine Combining

SESS Self-encoded Spread Spectrum

SLA Square Loop Antenna

SLG Single Layer Graphene

SNR Signal to Noise Ratio

SP4T Single Pole Four Throw

SSC Switch and Select Combining

TDMA Time Division Multiple Access

UAV Unmanned Aerial Vehicle 
UWB Ultra Wide Band

WBAN Wireless Body Area Networks

WLAN Wireless Local Area Network 



\section{Chapter 1}

\section{Introduction}

\subsection{Statement of topic and focus}

Wireless communication in the modern day is ubiquitous. The modern household today usually contains more than one wireless communication device on average. With the advent of affordable smart devices which communicate wirelessly, increased focus has been placed on creating applications that we use to share information with others as well as other devices. Therefore, our lives have become more dependent on this information that we share with, more than ever before.

Wireless communication technologies have also increasingly permeated into other aspects of our lives where traditionally, limited or no communication was possible. Service personnel such as fire fighting personnel, forestry officials, emergency health services personnel and defense personnel all communicate important mission critical information using wireless devices. RFID tags are now found embedded in the skin or collars of animals roaming in the jungles or swimming in the oceans. Therefore, wireless communication today has taken a far more pervasive role in our lives than was previously thought possible.

Due to this nature of carrying with us wireless communicating devices close to our bodies, developing technologies for body wearable communication devices has become an increasingly sought after topic among the antenna research community in recent times.

\subsubsection{Vision and motivation for the research}

In the last two decades, new body wearable communication devices have been designed and employed for everyday use with some success. Newer body wearable technologies now involve ways of improving the wireless communication aspect of these devices in order to provide cost effective but robust communication platforms even in the harshest of 
environments. In view this, I aim to look at ways of improving the communication methods used by service personnel who engage in mission critical scenarios, who communicate using such body worn antennas.

The modern soldier engaged in a battlefield scenario carries with him means of communication with his command center or his support troops. They provide him with updated threat analysis relayed to him via satellites, drones, spy planes etc and mission critical instructions or information. Efficient, clear and accurate communication in such scenarios can often be life saving for the personnel on ground.

However, there are various problems faced by military personnel on ground when using portable radio communication sets. Multipath propagation can lead to errors in the received signals during signal decoding or it can lead to the occurrence of echo during communication. The use of radio communication is sometimes subjected to jamming signals by enemy forces. Therefore, there is a need a to have an effective technique to avoid undesirable interference or jamming signals in such scenarios.

The weight of the communication equipment, especially the battery, can possibly impede the fast movement of the soldier which can become very critical in a battlefield scenario. Carrying less heavy and bulky equipment increases his mobility and therefore, his chances of survival, in a hostile environment. The limited battery power available to him and the inability to recharge the batteries in his communication equipment while out in the field over prolonged hours, severely restricts the use of his communication equipment.

Sometimes, communicating with your command unit far away requires your antenna to have a longer reach which may not be possible with some antenna types used in the radio equipment. Increasing the transmit power to transmit signals over a wide area is not only harmful to the user but it also severely drains the available battery power very quickly. This will limit the duration of communication he can have with his command center.

In some scenarios, the units on the ground may be moving very quickly (e.g. if travelling in a vehicle) while maintaining contact with other fast mobile units (e.g. drones overhead). This would mean maintaining a direct signal transmission between a mobile user and a mobile base station with random directions of movement. This requires fast signal tracking of a moving transmitter or a receiver.

Modern day battlefield scenarios involve the transmission of high quality images in intelligence gathering and pre-ops planning. These involve maps of enemy locations, thermal imaging maps showing hidden threats, HD video conference calls with those in distant command centres etc. Such communication formats require high throughput data links which are difficult to establish with conventional antennas, in rough terrain. It is preferable to have less signal bit error rates and less repetitive broadcasts due to time constraints in mission 
critical scenarios. Therefore, an important challenge in such scenarios is to provide error free high throughput communication links even in a weak EM environment.

Due to the need to be mobile in a hostile battlefield environment, lightweight communication equipment is preferred. Therefore, unobtrusive and conformal antennas worn on the body can be advantageous over long whip antennas carried on older communication radio sets.

Therefore, from an antenna research perspective there is potential to develop new technologies that involve conformal body wearable antennas which can increase the throughput, make use of battery power efficiently, provide longer communication range, track a fast moving mobile transmitter or receiver using beam steering capability.

\subsubsection{How we can locate it within broader work?}

By looking at the above topic and focus, I identified that the use of high Gain directive antennas can achieve several of the objectives described in the previous section such as: longer signal reach, efficient use of battery power, transmission in one direction only while avoiding jamming and interference in other directions, signal tracking and beam steering capability etc.

The use of directive antennas in signal tracking and beam steering is an established concept, even in the defence sector (e.g antenna arrays, radars). However, these antennas due to their size and complex power and electronic processing requirements, cannot be practically used by single mobile users in the battlefield scenarios. Therefore, there is a need to develop single unit beam steerable antennas which have simple beam steering or beam switching mechanisms and wide beam steering coverage. There are many examples of beam steerable antennas found in literature. However, a larger proportion of them have limited beam steering range. Square loop antennas with beam steering in the near $360^{\circ}$ azimuth range have been proposed earlier and is a well established concept. I aim to explore the possibility of employing such antennas as body wearable conformal antennas in this research work.

The requirement to provide antenna beam steering, user tracking and jamming avoidance can be achieved by various signal processing techniques combined together with electronic phase shifting components or RF switching components. There are many examples of beam steering algorithms or signal processing techniques used for jamming avoidance. Such methods are widely used in practice. However, Incorporating this beam steering and user tracking capability into such body wearable beam steerable antennas is rarely found in literature and is a novel concept. Body worn antenna previously found in literature included mostly non-pattern reconfigurable antennas, barring a few exceptions. 
Another advantage of using a highly directive pattern reconfigurable antenna beam, which can also track a signal of interest, is the efficient use of battery power and high throughput in communication resulting from less signal errors.

\subsubsection{Choice of research setting and data collection strategy}

In order to research, design and build the software controlled beam steerable body wearable antenna, I aimed to use both calculated (simulated) and experimental validation of the prototypes.

The 3D model of the conformal body wearable antenna was designed using the Microwave Design Suite ${ }^{\circledR}$ of the CST (CST, 2013). The designed model was then simulated using FDTD EM simulation by CST. The impedance matching, its return loss coefficient, its current distributions and Farfield patterns were thus viewed using CST and the 3D designs were optimized to redesign this model until the antenna beam patterns generated by EM simulations met the above requirements.

In order to design the conformal prototype of the body wearable antenna, a Kevlar ${ }^{\circledR}$ MK7 large sized helmet was procured. It was then scanned using a 3D scanner. This scan was then simplified to reduce the file size using various 3D image manipulation software such as 3DS Max $^{\circledR}$ (Autodesk, 2013), Geomagic Studio ${ }^{\circledR}$ (Geomagic, 2013) and Solidworks (Solidworks, 2013). Once a light watertight model was obtained, the 3D model was imported to CST MWS and the conformal antenna was designed and simulated once again.

The algorithm for the beam steering was developed using C\# language for a Windows ${ }^{\circledR}$ platform and using $\mathrm{C}$ language for a Linux platform.

The experimental data for the impedance matching was obtained using a Vector Network Analyzer (VNA) and the experimental Farfield Gain patterns were obtained using MVG's Satimo Starlab ${ }^{\circledR}$ Antenna Test Measurement Facility (MVG, 2014).

The experiment for the throughput analysis and beam steering was conducted indoors at the RF Antenna and Smart City Laboratory of the Swansea University where the experimental setup was placed in close proximity to several electronic equipment and furniture to mimic a high EM noise and multipath environment. Where the received signal strengths were too high to test the research hypothesis, I attenuated the transmit and receive signals by adding attenuators until the signal levels were weak enough to conduct the beam steering experiment. 


\subsection{Outline of this thesis}

The remainder of this thesis is organised as follows. The next section of this chapter lists out the peer-reviewed journals and conference publications relevant to the work presented in this thesis.

In Chapter 2, the background to the problem statement is described. The multipath propagation phenomena is defined and described briefly. Next, I look at the problems arising from multipath and the ways to mitigate multipath. Several techniques such as estimation methods, filtering, use of high Gain antennas, signal coding methods are described briefly.

This thesis then looks at what important characteristics of antennas aid in creating high Gain beams that help to avoid multipath. Important antenna measurement parameters such as Gain, Directivity, Array Factor, antenna efficiency are defined. Several advantages of using directive antenna beam patterns such as avoiding interference and jamming, signal range extension are illustrated and described next.

It then discusses the advantage of using directive high Gain antenna beams instead of omni-beam patterns in order to preserve battery power and make efficient use of the power resources in signal transmission. Finally, in Chapter 2 the gap in knowledge is identified and stated. The research problem statement is listed out next.

In Chapter 3, I look at the existing research work done in the area of pattern reconfigurable antennas, conformal body wearable antennas and beam switching algorithms. First, I look at how the pattern reconfigurable antennas have evolved over the past. I then review the current state of the art of the single unit pattern reconfigurable antennas available in literature. I have broadly categorised them into the different switching technologies used in the antennas. Then I look at other ways of designing pattern reconfigurable antennas such as FSS and HIS. I then review other work done in the area of pattern reconfigurable antennas, some of which involve non-planar structures. In this chapter, I discuss the suitability of each of the antenna type discussed in view of adapting them for conformal platforms.

The next section of Chapter 3 discusses pattern reconfigurable antennas designed for body wearable applications. Conformal and flexible body wearable antennas are then described and their advantages and the shortcomings are then identified. The last part of the Chapter 3 discusses different DoA algorithm types and beam switching schemes such as SC, SSC and SEC schemes previously found in literature. Chapter 3 then concludes by giving an overview of the designs and ideas discussed and their usefulness in implementing a solution for the problem statement mentioned in Chapter 2.

In Chapter 4, I present the preliminary research work and findings in designing and building a prototype of a conformal body wearable pattern reconfigurable antenna. I have presented the findings of several analytical studies conducted in the adaptability of the planar 
SLA to a conformal platform. The bending limits of the pattern reconfigurable SLA on doubly curved surfaces are illustrated by way of neat and distorted pattens, after observing the radiation pattern in each case. The extent of the radius extension, curvature extension and substrate thickness extension when the SLA was made conformal to a doubly curved surface is presented and the antenna's adaptability is discussed. A fabricated prototype of the final HSLA thus built is then presented along with its experimental and simulated radiation patterns and impedance response characteristics.

The second part of this chapter presents the preliminary work done in developing a beam steering algorithm. The algorithm code was written in C\# for a Windows ${ }^{\mathrm{TM}}$ based mobile device. Its function was tested using an experimental setup in which the HSLA was connected as the Base Station (BS) antenna while the roaming mobile device is moved around it. A HD video conferencing call application was run with an external server and its video call statistics were obtained. The results of this experiment are summarised in the last part of Chapter 4.

Chapter 5 presents the remaining experimental work done to validate the use of this HSLA in a noisy EM environment having multipath. The algorithm in chapter 4 has been modified and written in $\mathrm{C}$ language to suit a Linux based SBC hardware platform. The function of this algorithm has also been improved to include a novel SEC switching scheme studied in the Literature Review section of this thesis.

The last section of Chapter 5 then describes how this new algorithm running on a fully portable hardware platform that controls the beam steering of the HSLA was then tested as a Mobile Subscriber (MS) with a fixed BS scenario. The algorithm which scans and locks to the port receiving the highest RSSI when the MS is on the move, was put to test in a HD video call conferencing scenario similar to that of Chapter 4. By using the video call statistics and the RSSI obtained at each iteration of the experiment, it was demonstrated that an improvement of up to $7 \mathrm{Mbps}$ was possible when the RSSI levels were close to the noise floor. This chapter then discusses how it was possible to conduct the HD video conferencing call without interruption when the beam switching algorithm was used, compared to the case of the HSLA not running it, where no communication was possible (98\% packet loss scenario).

Chapter 6, discusses the preliminary work done in adapting this beam steerable antenna for higher frequency bands. It first discusses how the planar SLA previously presented in literature was scaled to a new frequency of $12 \mathrm{GHz}$ (Ka band). Its analytical results are then discussed and its shortcomings and areas for future work are identified. This chapter then presents how a SLA with High Impedance Surface (HIS) was then scaled according to a scaling factor using the old reference frequency and new frequency as a guide. The 
antenna presented, is planar SLA with a HIS for $25 \mathrm{GHz}$ ( $\mathrm{K}$ band). The chapter discusses how the preliminary analytical results are encouraging and outlines what improvements need to be done in future for this antenna. It then discusses how a third, $30 \mathrm{GHz}$ (Ku band) SLA was scaled from a previously published $4.7 \mathrm{GHz}$ planar SLA found in literature. Its analytical findings are presented and its shortcomings discussed, while areas for future work are identified once again.

The second part of Chapter 6 then discusses several ways of improving the beam switching algorithm presented in Chapter 5. It presents a simple, yet effective DoA algorithm presented in literature and studies how this can be adapted for the 4-beam SLA to increase the beam scanning coverage of the HSLA. Next, it discusses the usefulness of using SNR as a switching metric instead of RSSI in the algorithm mentioned. Finally, it makes an important suggestion to improve the beam switching algorithm presented in Chapter 5 by using a moving optimal switching threshold instead of the currently fixed switching threshold.

The concluding chapter of this thesis summarises the main ideas and work discussed in each preceding chapter. It then looks at how I was able to achieve the research objectives outlined in the problem statement in Chapter 1. 



\section{Publications relevant to this thesis}

1. H. Goonesinghe, A. Mehta, R. Lewis and N. Clow, "A conformal pattern adaptive Square Loop Antenna for body and vehicle platforms," 2014 IEEE Antennas and Propagation Society International Symposium (APSURSI), Memphis, TN, 2014, pp. 1445-1446. http://ieeexplore.iee.. rg/stamp/stamp.jsp?tp=\&arnumber=6905048\& isnumber $=6904322$

2. A. Mehta, S. Gautam, H. Goonesinghe, A. Pal, R. Lewis and N. Clow, "Planar pattern reconfigurable antenna integrated with a WiFi system for multipath mitigation and sustained high definition video networking in a complex EM environment," 2015 IEEE International Symposium on Antennas and Propagation \& USNC/URSI National Radio Science Meeting, Vancouver, BC, 2015, pp. 2229-2230. http://ieeexplore.ieee.org/ stamp/stamp.jsp?tp=\&arnumber $=7305503 \&$ isnumber $=7304376$

3. A. Pal, A. Mehta, H. Goonesinghe, D. Mirshekar-Syahkal and H. Nakano, "Conformal Beam-steering Antenna Controlled by a Raspberry Pi for Sustained High Throughput Applications," in IEEE Transactions on Antennas and Propagation, vol. PP, no. 99, pp. 1-1. http://ieeexplore.ieee.org/stamp/stamp.jsp?tp=\&arnumber=8126822\&isnumber= 4907023

4. D. Mirshekar-Syahkal, H. Goonesinghe, S. Gautam, A. Mehta, A. Pal and H. Nakano, "Conformal Beam Steering Antenna for Realising Adaptive Sensor Networks for High Throughput Applications in Weak Electromagnetic Environments", 2018 Microwaves, Antennas \& Propagation, IET, [Submitted in Oct 2017. Accepted with major corrections in Jan 2018]. 



\section{Chapter 2}

\section{Background}

\subsection{Introduction}

In this chapter we outline the background related to the problem being solved. The next section defines multipath propagation and its implications for wireless communication. Next, we look at the most common methods used in wireless communications to overcome the multipath problem in a Non Line of Sight (NLOS) wireless communication environment. We then look at how this problem affects the Defence industry with a focus on a mobile user trying to communicate with his ground units via a body worn antenna. We then look at ways on how to improve the communication quality by reducing the Signal-to-Noise Ratio (SNR) and provide an error free communication in a multipath rich EM environment. We then focus on what current technologies are available in order to overcome this. We then propose a novel, single unit, unobtrusive, conformal helmet antenna combined with a novel algorithm which enables it maintain a more robust communication like with the base station.

\subsection{Multipath propagation}

In Hargrave (2001) multipath propagation is defined as "the propagation of a wave from one point to another by more than one course". In RF signal propagation, multipath signals are produced by reflection, scattering and diffraction caused by obstacles in the wave's path when propagating towards the receiver. In addition to the LOS signal travelling direct from the transmitter to the receiver, there may also be unwanted replicas of the signal, which have travelled on longer paths, arriving at the receiver as mentioned in Alexandru and Elena-Simona (2015). 


\section{Background}

The resultant signal at the receiver in a multipath environment can be described below as shown in Fielitz and ter Haseborg (2014):

$$
h(f)=\sum_{i=1}^{N} a_{i}\left(\cos \left(\varphi_{i}\right)+j \sin \left(\varphi_{i}\right)\right)
$$

where $\boldsymbol{h}(\boldsymbol{f})$ is the received signal at frequency $f, N$ the number of different multipath or signal copies received, $a_{i}$ the amplitude of each multipath copy of the signal and $\varphi_{i}$ the phase of each signal copy due to the time delay in travelling via each multipath. $\varphi_{i}$ is given by the following formula, as shown in Fielitz and ter Haseborg (2014):

$$
\varphi_{i}=d_{i} 2 \pi f
$$

where $d_{i}$ is the time taken by the signal to arrive and $f$ the signal frequency.

Due to the time difference between the LOS signal and the NLOS signal copies, these out of phase signal copies can lead to errors at the receiver as shown in Fig. 2.1. Fig. 2.1 (Top) shows a signal waveform arriving at the receiver via the Line of Sight (LOS) path as well as four NLOS paths (NLOS 1 to 4). These signal copies are summed together to obtain the resultant signal at the receiver as shown in Fig. 2.1 (Bottom). The time between the detection of the LOS signal and the detection of the last multipath signal copy is called the wireless channel's delay spread.

The resulting signal's amplitude and phase is distorted due to the addition of the multipath copies.

\subsubsection{Problems caused by multipath}

Multipath in wireless communication can cause the following problems at the receiver:

- Errors in Direction of Arrival (DoA) estimation of received signal

- Inter-Symbol Interference (ISI)

- Partial/Total cancellation

- Reduced effective bandwidth

- Errors in time delay estimate of the Delay Lock Loop (DLL)

In wireless localization applications and satellite navigation systems such as the Global Navigation Satellite System (GNSS) or Global Positioning System (GPS), the Received Signal Strength (RSS) information and Direction of Arrival (DoA) estimates are used to 

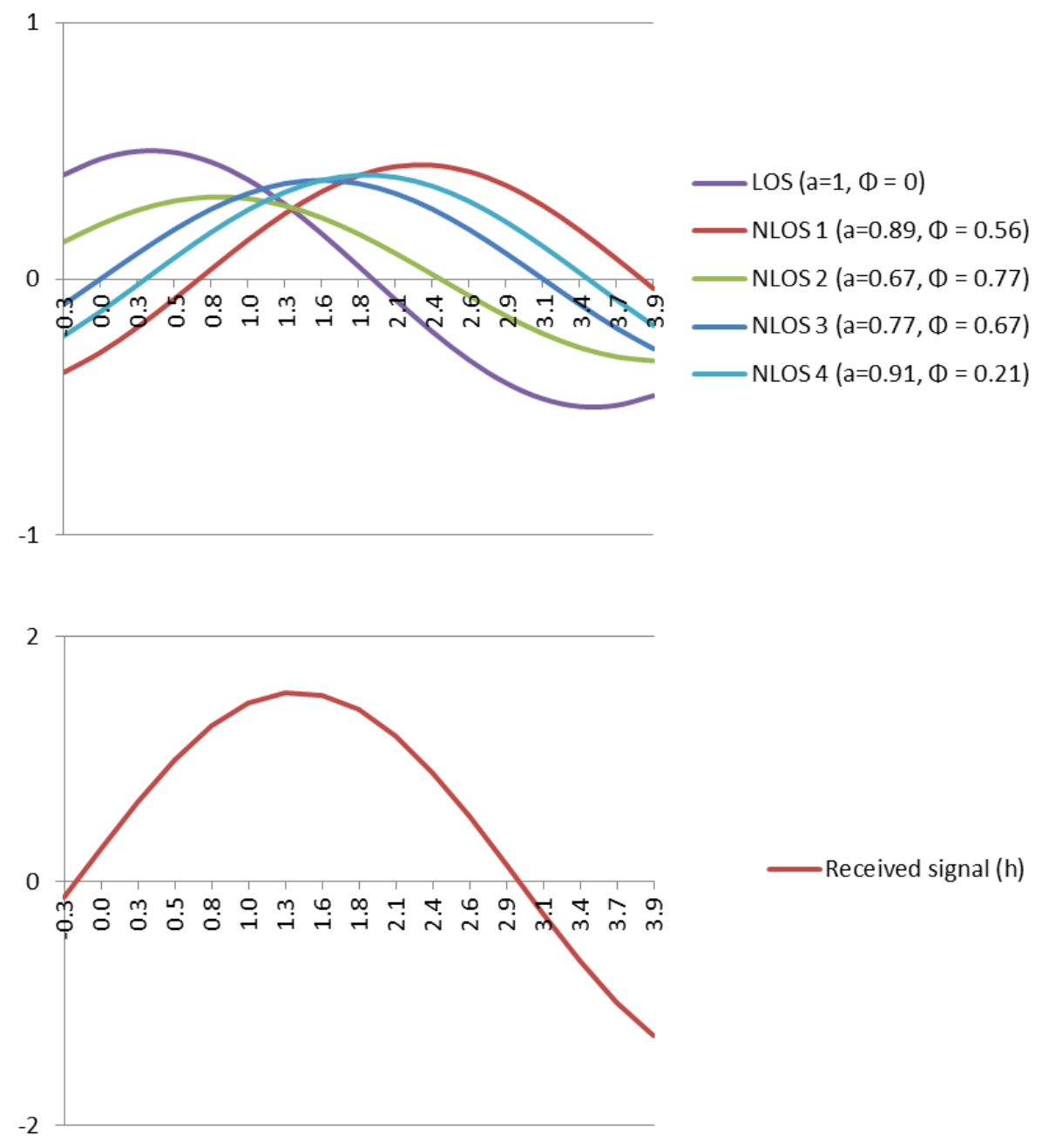

Fig. 2.1 The LOS signal and the NLOS signal copies arriving at the receiver (Top) and the resultant signal when they are combined at the receiver (Bottom) 


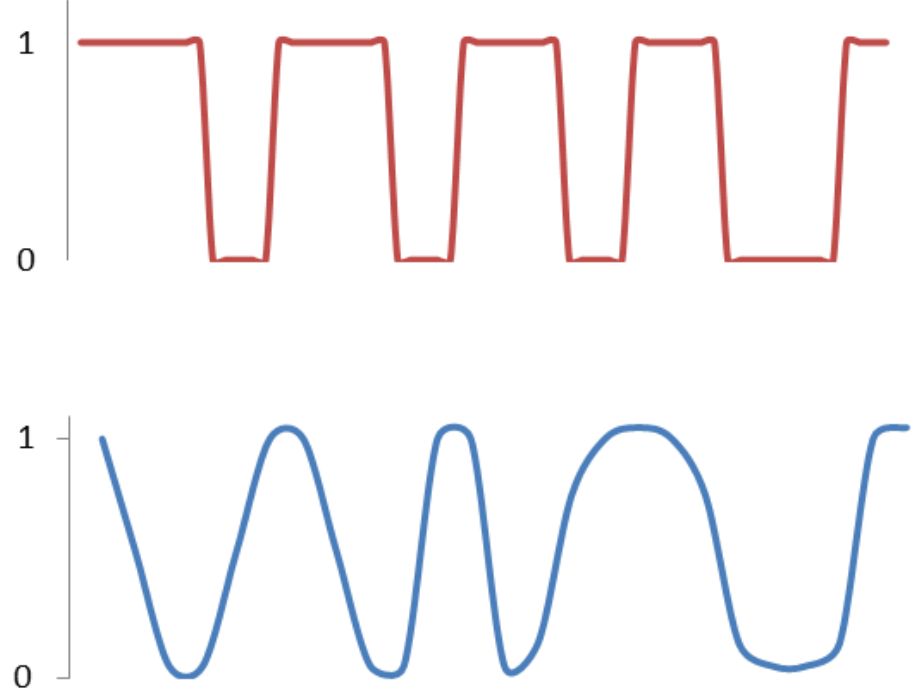

Fig. 2.2 The data transmitted (Top) and the signal received showing ISI due to multipath (Bottom)

determine the user location. Multipath signals arriving at the receiver cause fluctuations in the received signal which introduce errors in the estimation of the DoA. In GNSS, received multipath signals can distort the correlation function shape creating errors in the receiver position estimates.

Furthermore, the multipath copies of the signal can be partially co-related as they arrive from the same source. This makes it difficult to use statistical signal processing to filter out the coherent multipath interference signals and isolate the LOS signal in some applications.

The phase delays caused by signals arriving via multiple paths result in the information symbols carried by the signal smearing or distorting. This is known as ISI and is displayed in Fig. 2.2. ISI occurs when the symbol detection time of the receiver is comparable to the channel's delay spread as mentioned by Nicopolitidis et al. (2003). As the shape of the signal conveys information being transmitted, the occurrence of ISIS will result in errors at the receiver when demodulating the signal to obtain this information.

Multipath signals sometimes are subject to phase shifts and polarization reversals due to scattering. When these copies arrive at the receiver, they may add destructively with the LOS signal. This may result in a received signal that is either fully or partially cancelled due to the out of phase multipath signal copies. This is known as partial/total cancellation and is shown in Fig. 2.3.

When multipath occurs, the receiver circuitry need to employ error-checking to ensure that the data it received is correct. Wireless Local Area Networks (WLANs) employ a 


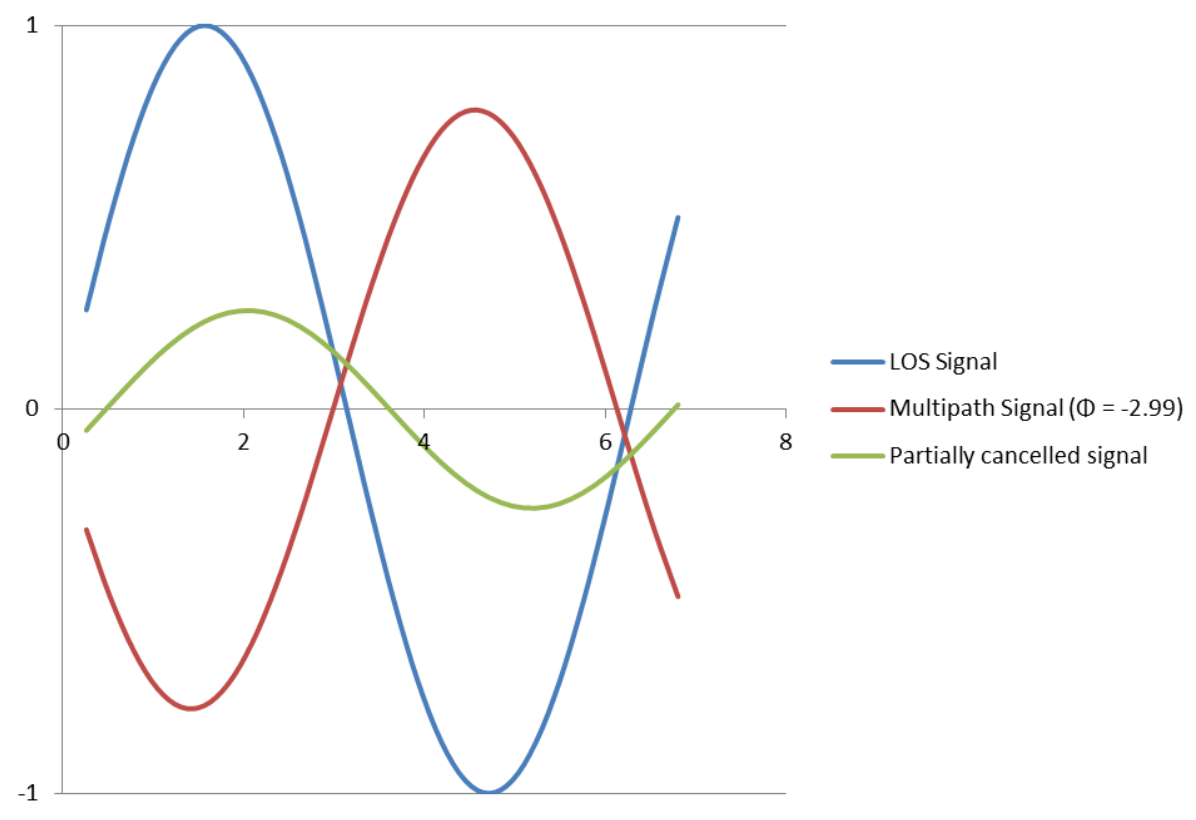

Fig. 2.3 The LOS signal, the out of phase Multipath signal and the partially cancelled received signal

protocol that requires the transmitter to retransmit the data if errors in data are detected at the receiver. These retransmissions reduce the effective bandwidth available to a user as mentioned in Geier (2015).

\subsubsection{Multipath mitigation}

There are several methods that are used to mitigate the issues caused by multipath. Some methods involve detecting the LOS signal and rejecting other multipath copies. These methods use statistical estimation to isolate the LOS signal using a priori information about the transmitted signal. When a priori information regarding the transmitted signal is not available, various filtering methods are employed at the receiver to mitigate the effects of multipath. The receiver circuitry then can use the filtered signal to recover the information that was transmitted. Other techniques involve physically spacing the antenna to spatially isolate the incoming signals from one direction. This method requires the use of directive antennas. Antenna beam-forming using antenna arrays are also used to direct the receiver antenna beam towards the transmitted signal's direction, in order to avoid multipath. Some signal coding methods have also shown to be more robust in their Bit Error Rate (BER) performance in multipath environment. 


\section{Statistical filtering}

When prior information about the propagation environment is available, filtering can be implemented at the receiver to filter out the multipath copies and accept only the LOS signal. Bayesian estimators such as maximum a posteriori (MAP) and minimum mean-square error (MMSE) methods are examples of statistical filters that are used to mitigate multipath.

In a fast changing propagation environment where the receiver and/or the transmitter is mobile, the noise induced by multipath can have an unknown probability density function (PDF). In such a scenario, filtering methods that depend on a priori information will not be effective in reducing multipath errors. Therefore, estimation methods such as the maximum likelihood estimators (MLE) or deconvolution based multipath mitigation techniques are used instead.

MLE estimate the channel parameters that maximize the PDF of the transmitted signal data during signal processing. While MLE approach has the advantage of approaching theoretical limits of performance, they are only useful in propagation channels with Gaussian noise environments. However, in fast changing multipath propagation environments, the PDF of noise is known to display Rayleigh Fading.

More advanced techniques for multipath mitigation have recently been proposed. For example, Marais et al. (2010) discusses the implementation of Jump Markov System (JMS) where the state of the multipath induced error at a GNSS receiver could be known a priori using a Markov model chain. In a closed simulation environment, JMS algorithm was shown to have a lower error rate and an increased accuracy in GNSS localization applications.

\section{Correlation/deconvolution-based multipath mitigation}

Some multipath mitigation techniques use DLLs in the receiver circuitry to estimate the channel parameters. Common techniques that use DLLs are the narrow correlator and the Early-Late Slope method. Use of DLLs in signal processing come at the cost of increased complexity and implementation cost at the receiver-end.

While the narrow correlator was one of the first methods used in signal processing for multipath mitigation, techniques that use adaptive filtering have later been proposed. Examples of these include the adaptive filtering process described by Rlinami et al. (2000) or the SNR-based adaptive estimation method described in Comp and Axelrad (1998).

In indoor wireless communication scenarios, the LOS signal may not be available due to obstacles such as walls. The LOS signal arriving through a concrete wall may be subject to severe fading compared to a NLOS signal. Hence, the strongest signal copy received at the receiver could sometimes be a NLOS signal. Many conventional algorithms that use 
correlator based approaches may fail to mitigate multipath in indoor multipath mitigation due to this reason. In Dragūnas and Borre (2010) two approaches, Projection Onto Convex Sets (POCS) and the deconvolution approach, are discussed as possible solutions for multipath mitigation for indoor GNSS receivers.

\section{Use of signal coding}

Although filtering techniques using signal processing are commonly used to mitigate multipath, some signal coding schemes have also proven to have better BER performance in multipath environments.

For example, Duraisamy et al. (2014) states that self-encoded spread spectrum (SESS), when combined with binary-offset-carrier (BOC) modulation scheme improved the navigational accuracy and reduced the tracking error in a GNSS system. Liu (2016) shows that OFDM-QPSK modulation scheme was shown to have better BER performance than normal QPSK in simulated multipath fading channel environment.

\section{Use of antennas}

In a multipath propagation environment where LOS signal is present, multipath mitigation can be achieved if the LOS signal can be isolated in space using an antenna. Antennas either transmit or receive EM signals. An antenna can be also considered as a filter which passes EM waves with certain frequency, polarization and arriving from a particular direction (Haupt, 2010). Therefore, to capture a signal coming from a particular direction, antennas with high Gain or antenna arrays combined with beamforming techniques can be used.

The Gain of an antenna can be defined as its ability to direct input power into radiation in a particular direction. Gain is measured at the peak radiation intensity and is usually measured in units decibel $(\mathrm{dB})$ or units $\mathrm{dBi}$, if the radiated power is compared with that of an isotropic antenna. Gain can be described as:

$$
\text { Gain }=4 \pi \frac{\text { Power radiated per unit angle }}{\text { Total power accepted from source }}
$$

The Directivity of an antenna is a measure of the concentration of radiation in the direction of the maximum. As mentioned in Milligan (2005), Directivity is related to Gain by the following equation:

$$
\text { Gain }=\frac{\text { maximum radiation intensity }}{\text { average radiation intensity }}=\eta \text { Directivity }
$$

where $\eta$ is the antenna efficiency. 


\section{Background}

While an antenna with high Gain is useful in capturing a signal of interest from a known direction, a LOS signal will not always be available in some wireless communication scenarios. Furthermore, an antenna with a Gain in a particular direction will capture other stray signals coming from that direction as well. Sometimes, the signal arriving at the receiver will also have undergone polarization changes due to reflections from obstacles. In such scenarios, the polarization of the receiver antenna is also of interest when mitigating multipath.

As defined by Milligan (2005), "the polarization of a wave is the direction of the electric field". An EM wave consists of an orthogonal Electric Field $(\mathbf{E})$ and a Magnetic Field $(\mathbf{H})$ and it travels in a direction perpendicular to both the $\mathbf{E}$ and $\mathbf{H}$ fields. As mentioned in Milligan (2005) the E-Field of a wave propagating along $z$-axis can be represented as:

$$
\mathbf{E}=E_{x} \hat{\mathbf{x}}+E_{y} \hat{\mathbf{y}}
$$

This can be rewritten as:

$$
\mathbf{E}=E_{x}\left(\hat{\mathbf{x}}+\hat{\rho_{L}} \hat{\mathbf{y}}\right) \quad \hat{\rho_{L}}=\frac{E_{y}}{E_{x}}
$$

where $\hat{\rho_{L}}$ is a complex constant denoting the linear polarization ratio.

If $\mathbf{E}$ is represented in time and the maximum of $\mathbf{E}$ is plotted over time on the XY plane, it will take the form of an ellipse rotating either clockwise (CW) or counter clockwise (CCW). This plot of the maximum of $\mathbf{E}$ over time is shown in Fig. 2.4. When the linear polarization ratio $\hat{\rho_{L}}=1$ or $0 \mathrm{~dB}$, the plot would take the form of a circle. When the phase difference between $E_{x}$ and $E_{y}$ is zero, the EM wave is said to be linearly polarized.

Circular polarization of an EM wave occurs when the perpendicular $\mathbf{E}$ field components $E_{x}$ and $E_{y}$, are of equal amplitude (i.e $\hat{\rho_{L}}=1$ ) and are $90^{\circ}$ out of phase.

In scenarios where the signal of interest can arrive from an arbitrary direction, the correct physical orientation of a high Gain antenna will be impossible to achieve. In such a scenario, using a circularly polarized antenna can be advantageous over a linearly polarized antenna. Designing a CP antenna over a wide bandwidth can be a challenging task compared to designing linearly polarised antennas. Therefore, in practice, an antenna whose radiated E-field displays $\hat{\rho_{L}}<3 \mathrm{~dB}$, is considered to be circularly polarised.

Use of antenna polarization property to mitigate multipath is quite common in systems such as GNSS. GNSS receivers use RHCP antennas for the LOS signal. They are able to reject multipath signals which, when reflected once, will become Left hand circularly polarized (LHCP). For example, Gafarov et al. (2016) presents a quadrupole GNSS antenna receiver with a spatial cylindrical polarizer for GNSS applications. Khosravi et al. (2015) also 


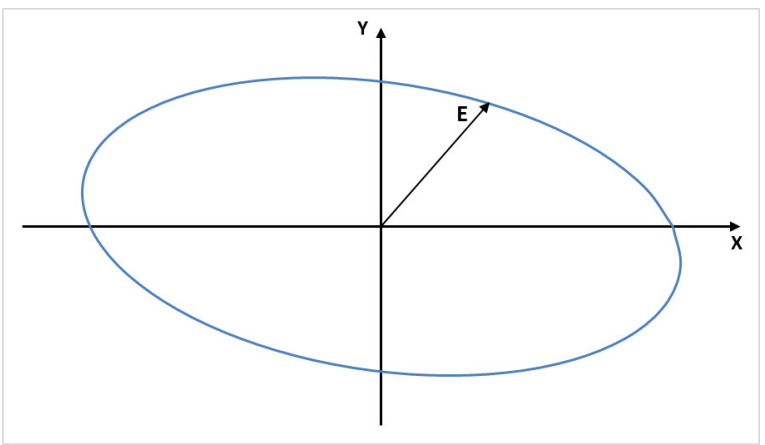

Fig. 2.4 Polarization Ellipse obtained by plotting maximum of $\mathbf{E}$ over time

presents a GNSS antenna which is designed in close proximity to a non-resonant polarisation selective surface, which can be used for multipath mitigation.

Antenna ground planes also play an important role in mitigating multipath reflections from obstacles below the antenna radiation pattern horizon. While the antenna ground plane creates a stabilizing artificial environment on which the antenna rests, it also becomes a part of the antenna structure and effects its radiation pattern. The stabilizing effect that the ground plane has on the antenna will vary with ground plane size. Using a large ground plane (larger than one wavelength in size) is a useful way of mitigating reflections from low angles, especially when the antenna is fixed on elevated ground.

For example, Yang et al. (2016) presents a design for an annular slot loaded ground plane antenna for GNSS receivers which can mitigate multipath. Zhang et al. (2016) presents a dual band GNSS antenna for 1.575 GHz (L1 Band) and 1.268 GHZ (B3 Band) which uses an Electromagnetic Band Gap (EBG) instead of a standard ground plane. It is shown that, the integration of the EBG into this dual band antenna greatly improves the multipath mitigation from reflections below the horizontal plane.

The disadvantage of using antennas with high Gain in one direction is the static nature of such a wireless communication link. A wireless communication link consisting of two high gain antennas transmitting in the direction of their highest Gain, will not compensate for changes in their relative position, for example when the transmitter or receiver is mobile, or the changes to obstacles in their LOS path. A possible solution for this is to use antenna arrays combined with beamforming to steer the beam in the direction of the transmitter.

A larger antenna will be able to receive more EM energy compared to a smaller antenna. Similarly, many individual antennas can be combined to form an antenna array, in order to receive more EM energy in the form of waves. Therefore, antenna arrays are used in situations where faint signals coming from far away sources need to be detected or conversely, when the signal needs to be transmitted over a longer range. 


\section{Background}

In an antenna array consisting of isotropic radiating antennas, the average power density, $S_{i}$ calculated from a distance $R$ from one isotropic radiator can be described as:

$$
S_{i}=\frac{P_{t} G_{t} L}{4 \pi R^{2}}
$$

where $\mathrm{L}$ is the loss factor $(L<1.0)$. Therefore, $S_{i}$ is the total radiated power of the isotropic radiator, divided by the surface area of a sphere. When an antenna receives an EM signal, the current induced by the incident wave can be expressed as a current density function which is distributed over an area called the collecting aperture $\left(A_{c}\right)$. The power then delivered to the terminals of the receiver antenna $P_{r}$, is equal to the incident power density multiplied by the effective aperture $\left(A_{e}\right)$ as mentioned in Haupt (2010). This can be be represented as:

$$
P_{r}=S_{i} A_{e}
$$

The effective aperture $\left(A_{e}\right)$ is also related to Gain $(G)$ by:

$$
G=4 \pi \frac{A_{e}}{\lambda^{2}}
$$

Haupt (2010) states that the effective aperture $\left(A_{e}\right)$ is a term related to receivers, whereas Gain $(G)$ describes both transmitter and receiver antennas. It follows from equations 2.7 and 2.8, that the power received by a receiver antenna $\left(P_{r}\right)$ at a distance $R$ from a transmitter antenna with Gain $\left(G_{t}\right)$ and transmitted power $\left(P_{t}\right)$ can be given as:

$$
P_{r}=\frac{P_{t} G_{t} L A_{e}}{4 \pi R^{2}}
$$

Equation 2.10 is known as the Friis Equation. According to Sevgi (2003) the radiation pattern of an antenna array depends on several factors: the individual elements, their relative location, the spacing among them, their orientation, their individual excitation amplitude and phase. However, the radiation pattern of an antenna array can be divided into two aspects: the individual element pattern and array factor (AF). Sevgi (2003) states that, array factor is the radiation pattern of an array consisting of entirely isotropic radiators. Isotropic radiators are hypothetical, lossless antennas, representing a point source suspended in space, which radiate uniformly in all directions.

Fig. 2.5 shows a linear antenna array consisting of 4 isotropic radiators spaced by a distance $\boldsymbol{d}$ along the $\mathbf{X}$-axis. The isotropic radiators each have identical spherical radiation patterns, which are repesented by the 4 dashed circles in the X-Y plane, numbered 1 to 4 . Four parallel incident waves along the $\mathbf{X}-\mathbf{Y}$ plane, $r_{1}$ to $r_{4}$ are shown arriving at each of the isotropic radiator antennas with an incident angle $\theta$. When $\theta=90^{\circ}$, all 4 incident waves 


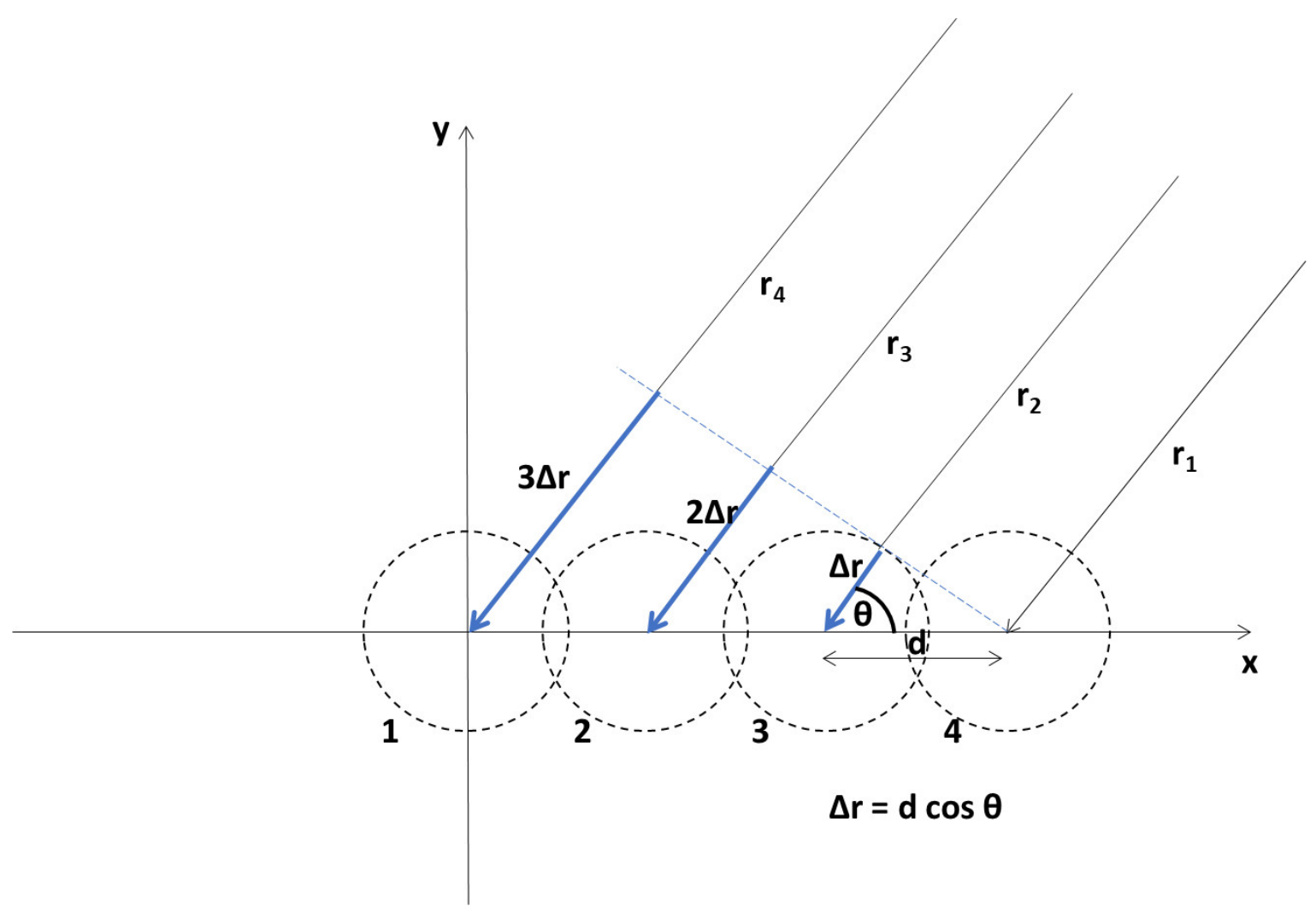

Fig. 2.5 A 4-element linear antenna array with isotropic radiators 


\section{Background}

travel paralle to the $\mathbf{Y}$-axis and hence, will travel equal distances and have the same phase. When $\theta \neq 90^{\circ}$ each incident ray has a path difference of $d \cos (\theta)$ relative to the previous radiator. Therefore incident rays $r_{2}$ through $r_{4}$ have path differences of $d \cos (\theta), 2 d \cos (\theta)$ and $3 d \cos (\theta)$ respectively. A path difference of $d \cos (\theta)$ corresponds to a phase difference of $\left(\beta=k_{o}\right)$ at the given frequency, as mentioned in Sevgi (2003).

$$
\text { Phase difference }=\beta d \cos (\theta)=\frac{2 \pi}{\lambda} d \cos (\theta)
$$

Therefore, if the relative phase differences between each array element can be adjusted, the antenna array's beam can be electronically steered in space.

The signals received by each antenna element in an array must be combined coherently (i.e. having the same phase) in order for their sum to be maximum. Therefore, in an antenna array, a feeding network is used to combined signals, from all antenna elements. Using antenna arrays come at the cost of increased cost, complexity and increased physical space.

Using an antenna array is advantageous over using a single antenna due to a few reasons. Interference rejection or multipath rejection can be achieved by weighting the signals received from each element, before combining them to obtain the received signal. The array's beam can be steered in space to different direction, without having to physically move the antennas. For example, Ong and Tan (2015) has shown experimentally how antenna arrays combined with Forward Backward Spatial Smoothing (FBSS) improves the multipath mitigation and improves the performance of a Direction Finding linear antenna array. Engelbrecht et al. (2016) also shows how the multipath mitigation performance can be improved by using an 8-element antenna array, using a phase-of arrival ( $\mathrm{PoA})$ ranging technique to derive the distances between mobile user and a fixed base station.

\subsection{Military communication in multipath environments}

In modern warfare, defence organizations deploy their units at multiple and diverse ground locations. Often, the operational command centres are located away from the battle field, requiring the ground units to communicate with them wirelessly, in real-time. If the soldiers on ground stay connected with their command center, they can be provided with updated threat analysis, critical instructions, information regarding support troop deployment and medical advice to treat battle wounds to help save the soldiers' lives. Therefore, it is important that the soldiers in the front line are able to establish a direct uninterrupted communication link using the high speed communication equipment worn on their on bodies and on the vehicles. The advancement of military communication equipment in recent times mean that secure communication links can be established via satellites or UAVs. which transmit 
messages back and forth between both parties. The availability of high bandwidth means that live video feeds are increasingly used to update the command centres about the ground situation via UAVs.

Wireless signals transmitted by military vehicles, planes, UAVs and on-body radios in weak battlefield environments such as deserts and mountainous regions, are subject to propagation environment effects such as multipath, diffraction, scattering and fading. The wireless channel in which they communicate in is often considered as time varying because, often, both the transmitter or the receiver can be moving, fast. Due to this reason, multipath mitigation techniques such as MLE discussed before can be ineffective as the transmitter or receiver travels at a high speed, as in the case of UAVs or fighter jets. Antenna arrays can be implemented where platform space is available (e.g on a plane or a military vehicle). However, antenna arrays are difficult to implement on on-body military communication equipment such as helmets or backpack radios used by ground units due to their demands on power and space. Military personnel prefer lighter, less bulky and unobtrusive communication equipment in order to stay highly mobile on the battlefield, while still being able to stay in contact with their operational command centres.

\subsubsection{Jamming Avoidance}

The increased use of military communication radios by military personnel, randomly deployed over unsecure and open hostile environments make them susceptible to interference and jamming by interested parties. Efforts have been made in terms of licensing special frequencies for military communication and the fixed allocation of spectral resources, to minimise such interference. Toole and Fok (2016) mentions that, such efforts will reduce efficiency, flexibility, and scalability. Therefore, it is necessary to utilize effective methods to protect communication links against malicious jamming.

Antennas or antenna arrays with adaptive radiation patterns can be useful in such scenarios to steer the beam away from an interfering source or a jamming signal, if such a source can be identified using signal processing.

\subsubsection{Improving range and battery life}

Due to weight constraints on equipment that can be carried by highly mobile military units, rechargeable batteries are used to power the military communication radios. Such batteries impose limitations on the transmitting distance for the communication radios as the transmitted power is limited due to the types of batteries used. Furthermore, the duration of communication can be affected due to the available battery power, if the battlefield 


\section{Background}

environment does not allow the radio batteries to be charged after a few hours. Therefore, using narrow beamwidth high Gain antennas are preferred over omni-directional antenna to save power and to transmit over long distances or capture faint signals.

\subsection{Problem statement}

In order to improve the efficiency of communication by mobile users using body wearable antennas, such as the helmet antennas worn by military personnel, a multifaceted approach is needed in hardware and software design. Several problems such as multipath mitigation, jamming and interference signal avoidance, signal transmission range extension, efficient use of limited battery power etc. need to be considered when designing such an efficient communication method while also focussing on the space requirement, mobility, battery life and power requirements of the proposed solution.

Due to the nature of body worn antennas, they need to be conformal to the surface of the platform they are implemented on (e.g. uniform fabric or combat helmet). Establishing a robust communication link providing a high bandwidth in a weak EM propagation environment is necessary to provide High Definition (HD) video quality feed for the operational command centres. Therefore a steerable antenna beam of high Gain is required to avoid multipath and interference signals. A software running a signal processing algorithm is needed to analyse the received signal and steer the beam towards the transmitter/receiver when high noise is present. The proposed solution needs to be simple, light and have a simple feeding mechanism and a software backend for processing the received signal.

\subsection{Proposed solution}

Multipath effects can be mitigated by spatially isolating the signal of interest using a smart antenna system. Single element beam steering antennas are gaining popularity for defence implementations. They are compact, cheap and don't need complex feeding mechanisms. A beam steering antenna coupled with an intelligent algorithm and a fast switching network allows the user to isolate a signal of interest and direct the receiver antenna beam towards the target only. This enables the communication system to mitigate multipath effects, achieve high throughputs and evade jamming.

In order for this hardware solution to be worn on body, the antenna needs to be conformal to existing accessories worn by the service personnel. Hence, the conformal and unobtrusive nature of the antenna need to be considered in the solution to be designed as well. 


\section{Chapter 3}

\section{Literature review}

\subsection{Introduction}

In this chapter I take a comprehensive look at the previous work done, in relation to the problem statement highlighted in Chapter 2. I first look at how pattern reconfigurable antennas have evolved over the past. Then I look at the state of the art of the single unit pattern reconfigurable antennas published in recent years. Next, I look at the suitability of some of these single unit pattern reconfigurable antennas as a possible solution to the problem to be solved, as described in Section 2.4. I then look at the work done in the areas body wearable or conformal helmet antennas. Finally, I investigate the different types of the beam switching algorithms and switching combining schemes published, which can be applied as a solution for steering the antenna beam towards a moving transmitter/receiver.

The last section of this chapter reviews the main work discussed on single unit pattern reconfigurable antennas, their suitability for mounting on conformal platforms and the beam switching algorithms that can be applied with them. It concludes by discussing the merits of the chosen solution.

\subsection{Reconfigurable antennas}

A reconfigurable antenna is one that has the capability to modify one or more of its parameters such as frequency, polarisation, beam shape, beam direction or tilt etc., without effecting its other properties. Reconfigurable antennas are difficult to design due to the different antenna parameters being closely linked to each other. Therefore, when one antenna parameter is changed, it often results in effecting the other parameters. 


\subsubsection{A historical perspective}

Most of the early work published on the topic of pattern reconfigurable antennas were mainly for the use of the space industry. Many of these examples included pointing a satellite antenna array's directive beam towards a receiver on earth, or vice versa. A closed loop feedback system was then used for electronically steering the beam using phase shifters. For example, the antenna array presented by Cooper and McComas (1960) consisted of slot radiators and operated in the $K_{a}$ band. Ghose (1964) presents a transmitter $\left(T_{x}\right)$ and receiver $\left(R_{x}\right)$ array system for space applications, where the beam steering is controlled using a PLL. The $T_{x}$ adjusts its beam direction according to a pilot signal transmitted from $R_{x}$. Mikenas et al. (1975) discusses the beam steering capability of a 16-element phased array of antennas operating in the 4.4 - 4.6 GHz range (C-band). In this array, phase shifters are used to steer the beam towards the $T_{x}$. The work published by Fetterolf and Price (1985) also consisted of a multi-beam antenna array designed for C-band. However, the adaptive beamforming network for this system comprised of a microprocessor controlled algorithm with a closed loop feedback system.

Ogawa et al. (1983) presented an adaptive array system which used an open-loop instead of a closed loop system for estimating the weights required for the beamforming. Clarricoats et al. (1988) has presented an offset mesh reflector antenna for spacecraft. This antenna increases the antenna beam's steering coverage using reconfiguration of the reflector surface.

Ding et al. (1995) presented the concept of using a semiconductor surface illuminated by a light source to generate a conducting area in order to create a reconfigurable antenna with it. The illuminated area was then connected to a MW signal source to create a radiating antenna. The reconfigurability of this an antenna was achieved by using a LCD screen or a similar light source which can be programmed to illuminate varying areas on the semiconductor material, thereby generating a reconfigurable patterns. This concept, as presented in Ding et al. (1995) is shown in Fig 3.1.

The reconfigurable antenna discussed in Freeman et al. (1992) is a 4 segment monopole antenna, whose segments are connected by 3 optoelectronic switches. By switching these 3 switches sequentially, each segment of the monopole can be connected to the previous one, thereby extending the length of the antenna and lowering its operating frequency at each step. Therefore, the antenna can operate in 4 possible frequency bands (multiband), each activated by the optoelectronic switches. The frequency reconfigurable antenna, presented by Freeman et al. (1992) is shown in Fig. 3.2. A similar concept was used in the antenna presented in Qian et al. (1999) where MEMS switches were used instead to create a reconfigurable (multiband) antenna. Later work by Linden (2001) has presented a grid patterned programmable antenna consisting of 30 mechanical relay switches and 1 feeding point. 


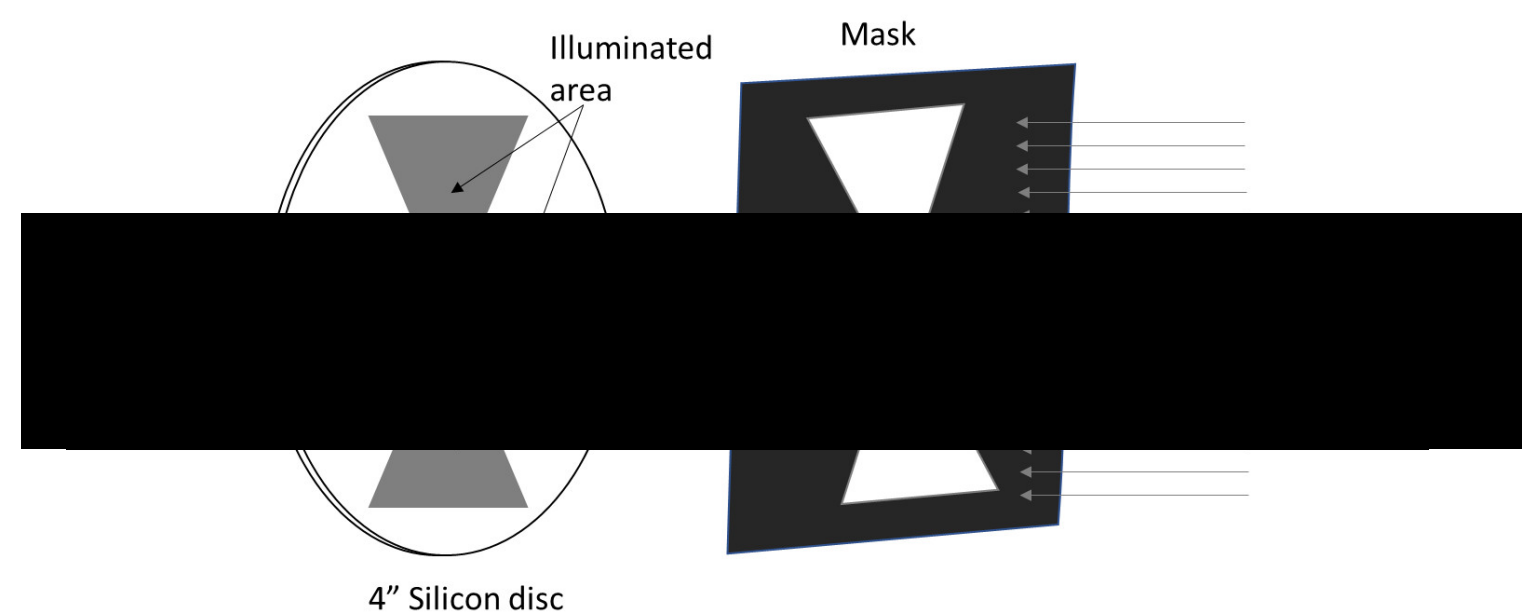

Fig. 3.1 Reconfigurable antenna using a semiconductor illuminated by a light source as presented in Ding et al. (1995)

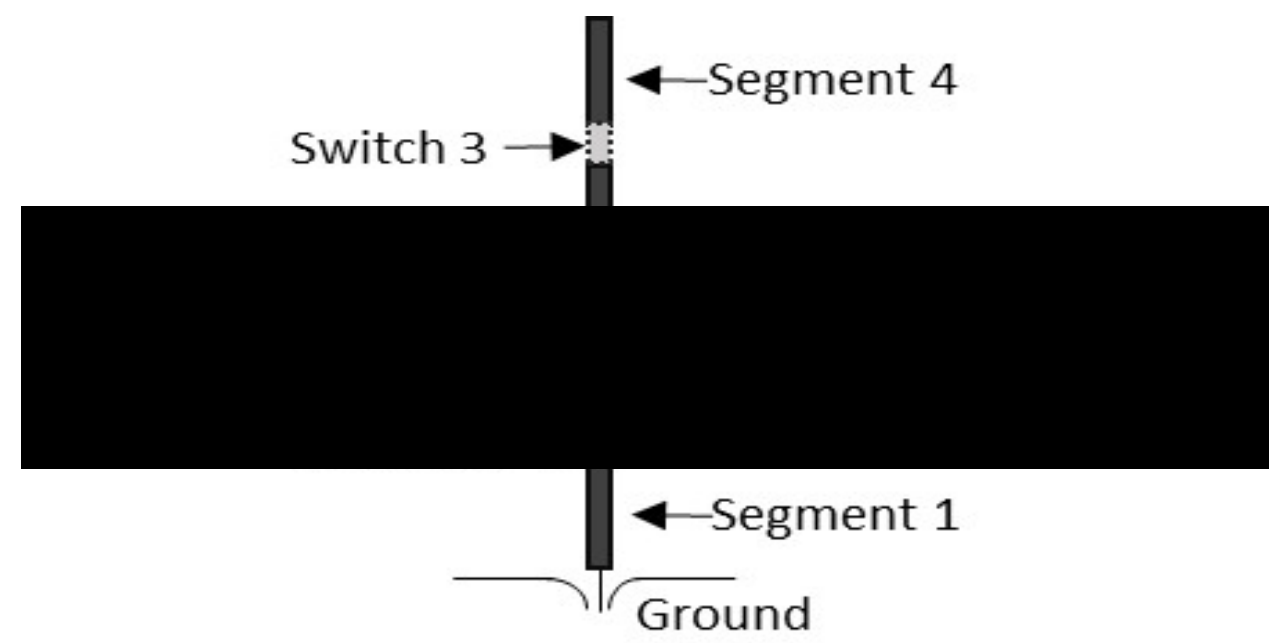

Fig. 3.2 4-segment monopole antenna using optoelectronic switches, as presented in Freeman et al. (1992) 


\section{Literature review}

With the advent of mobile communications, the interest in reconfigurable antennas shifted to mobile base station antenna arrays and reconfigurable single unit antennas for portable devices. Some of the earliest work reported on pattern reconfigurable antennas for mobile base stations was the cylindrical slot antenna array presented in Hirokawa et al. (1992) and the large cylindrical patch array antenna for mobile stations in Alakija and Stapleton (1992). Cupo et al. (1997) has also proposed a 4-element array for the IS-136 PCS base station using TDMA.

The mobile base station antenna proposed by Ebine and Ito (1997) consists of two radiating elements surrounded by vertical side reflectors, which will reduce the sidelobe levels. This dual beam antenna is smaller in size $(0.75 \lambda)$ compared to a $2 \times 2$ array. It therefore minimises the space required on a base station antenna. Angelucci et al. (1994); Armogida and Peixeiro (1997); Chang et al. (1999); Dobias and Gunther (1995); Johannisson (1998) have also proposed various beam-switching techniques in the past, for beam reconfigurable antenna arrays for mobile base stations.

Later work on reconfigurable antennas revolved mostly around reconfigurable antennas developed for mobile device applications at lower frequency bands. Wahid et al. (2003) presented a reconfigurable Yagi antenna constructed using two antennas superimposed on each other and separated by a p-i-n diode switch. They operated in the $2.4 \mathrm{GHz}$ and 5.78 $\mathrm{GHz}$ frequencies. The antennas presented in Boisbouvier et al. (2002); Kang et al. (2010); Lim and Ling (2007); Xiao et al. (2003) also follow a similar concept to the one presented in Wahid et al. (2003), where the pattern reconfigurability of the antennas, are obtained using $\mathrm{p}-\mathrm{i}-\mathrm{n}$ diodes in the antenna structure. The pattern reconfigurable antenna for the L1 frequency band, presented by Lee et al. (2006) involves a square patch antenna surrounded by a square microstrip conducting loop loaded with switches. The antenna's RHCP radiation pattern is reconfigured using the switches on the outer ring.

The cubic-slot antenna presented in Sarrazin et al. (2009) has 6 different radiation pattern combinations given by the diodes across the slots in each face of the cube. This antenna structure is shown in Fig. 3.3.

Other reconfigurable antennas presented in previous work have frequency or polarization diversity. For example, Yang and Rahmat-Samii (2002) has presented a polarization reconfigurable patch antenna which is configured using a diode, in the $4.64 \mathrm{GHz}$ frequency. The

spiral antenna presented by Huff et al. (2003) is both pattern reconfigurable and frequency reconfigurable. It operates in either $3.4 \mathrm{GHz}-4.0 \mathrm{GHz}$ band or the $5.6 \mathrm{GHz}-6.3 \mathrm{GHz}$ bands depending on the configuration of the switches.

The use of metasurfaces in reconfigurable antennas have also become increasingly popular in the last few years. A metasurface includes a 2-dimensional distribution (an array) 


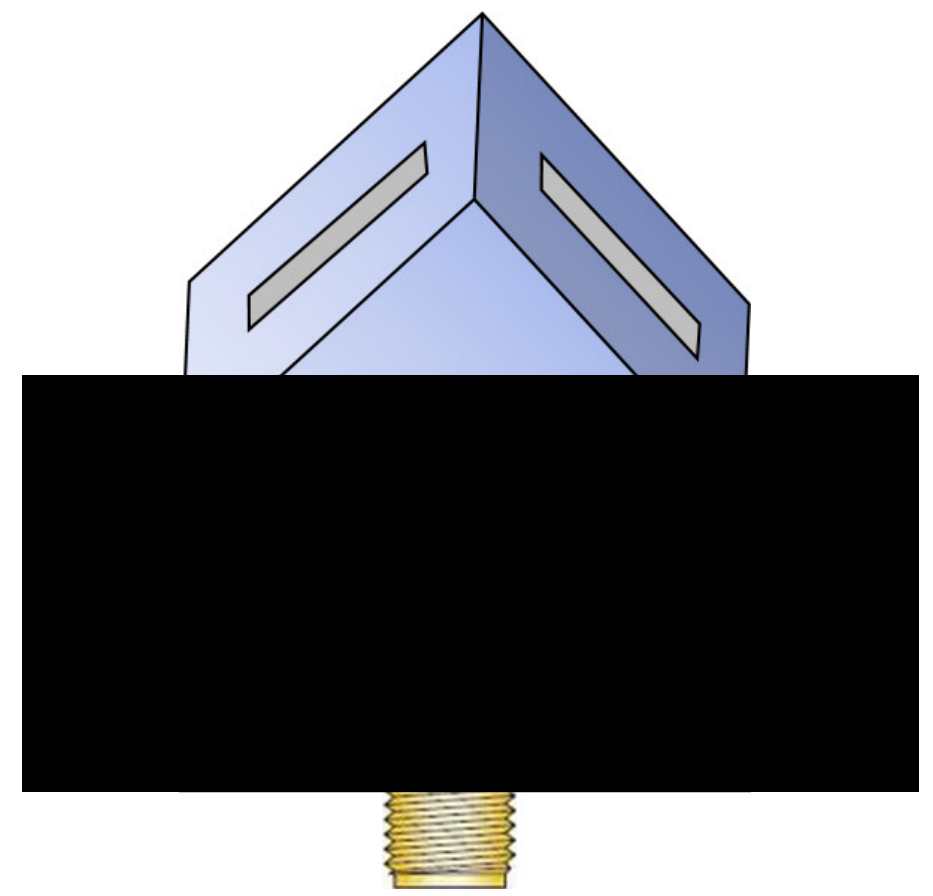

Fig. 3.3 Pattern reconfigurable cubic antenna as presented in Sarrazin et al. (2009)

of electrically small scatterers embedded in the material. Such metasurfaces are used as the substrates for the reconfigurable antennas discussed in Ali and Biradar (2016), Wu et al. (2016), Jnana and Sheeba (2015), Zhu et al. (2014a) and Zhu et al. (2014b).

The latest work on reconfigurable antennas increasingly involved single unit pattern reconfigurable antennas. These will be discussed in detail in the next section.

\subsection{Pattern reconfigurable antennas using Diodes and switches}

According to Bernhard (2007) pattern-reconfigurable antennas are antennas with high directivity, which can switch their antenna beams towards different directions according to system requirements. As discussed in Chapter 2, directional antennas use less energy, have signal sensitivity and have a longer range compared to omnidirectional antennas. A single unit pattern reconfigurable antenna consists of one antenna (single radiating element) instead of an array of antennas. The radiation pattern or the beam direction can be reconfigured in a pattern reconfigurable antenna by altering various properties of the antenna or using different feeding points within the structure. This section reviews several single-unit pattern reconfigurable antennas that were reported in literature in recent years.

Use of a p-i-n diodes for connecting different radiating elements in an antenna and thereby altering the antenna aperture size, is a popular method for creating pattern reconfigurable 
reconfigurable antennas. The forward biasing of a $\mathrm{p}$-i-n diode will result in the creation of a direct electrical path connecting two separate radiating elements, resulting in a reconfigurable radiation pattern in the antenna depending on the ON/OFF state of the p-i-n diode. Several such antennas that operate with $\mathrm{p}-\mathrm{i}-\mathrm{n}$ diodes have been reported in recent times.

The reconfigurable square slot antenna reported by Valizade et al. (2012) uses a p-i-n diode biasing method for obtaining additional resonances to cover the full UWB range from 3.1 GHz to $10.6 \mathrm{GHz}$. When the p-i-n diode is OFF, it creates a band-rejection in the frequency band from $5.03 \mathrm{GHz}$ to $5.94 \mathrm{GHz}$. This is useful in limiting its interference with other wireless communication devices operating at $5.2 \mathrm{GHz}$. Although, this antenna produces reconfigurable radiation patterns at higher frequencies as well as when $\mathrm{p}-\mathrm{i}-\mathrm{n}$ diode is in $\mathrm{ON}$ or OFF states, its directivity deteriorates at higher frequencies.

Qin et al. (2012) has proposed a U-slot antenna with 3 different radiation pattern configurations driven by $8 \mathrm{p}$-i-n diodes. This antenna has $8 \mathrm{p}$-i-n diodes and 8 shorting vias connected to ground, around the square patch antenna. The $\mathrm{p}-\mathrm{i}-\mathrm{n}$ diodes connect the shorting vias to the edge of the square patch. This antenna design is shown in Fig. 3.4 (Top). This antenna has 3 configurations. When the $4 \mathrm{p}$-i-n diodes on the two sides parallel to the Y-axis are forward biased, while the remaining 4 are reverse biased, this configuration will result in a conical shaped pattern, tilted along the Z-Y plane. This pattern is shown in Fig. 3.4 (Bottom). When the $\mathrm{p}-\mathrm{i}-\mathrm{n}$ diodes on the remaining two sides are forward biased while keeping the rest reverse biased, this will result in a radiation pattern with a slight null along Z-axis. When all 8 p-i-n diodes are zero biased, this will also result in radiation pattern similar to configuration-II, but in the boresight direction. Hence, this antenna will have 3 different beam patterns. However, only one pattern (configuration-I) has a directive beam. In addition, it will use 8 p-i-n diodes which will need to be driven by control voltages for the 3 configurations described.

Abutarboush et al. (2012) has also presented a wideband reconfigurable slot antenna which uses two radiating patches. The $50 \Omega$ microstrip line used for feeding the antenna is connected to two microstrip lines orthogonal to the feeding line. They are then connected to the two radiating patches via $2 \mathrm{p}-\mathrm{i}$-n diodes. This combination of two diodes results in 3 possible antenna configurations: OFF-ON, ON-OFF and ON-ON. The resulting radiation patterns for each of these 3 states, have good directive beam patterns and their impedance bandwidth is from $5 \mathrm{GHz}$ to $7 \mathrm{GHz}$. The pattern reconfigurable antenna for $2.4 \mathrm{GHz}$ as reported in Lai et al. (2009) also uses 2 p-i-n diodes to reconfigure its beam pattern. It is printed on a 2-layered PCB board and its two diodes are biased as either ON-OFF and OFF-ON. The resulting radiation patterns are similar for the two states but are orthogonal in the azimuth direction. 


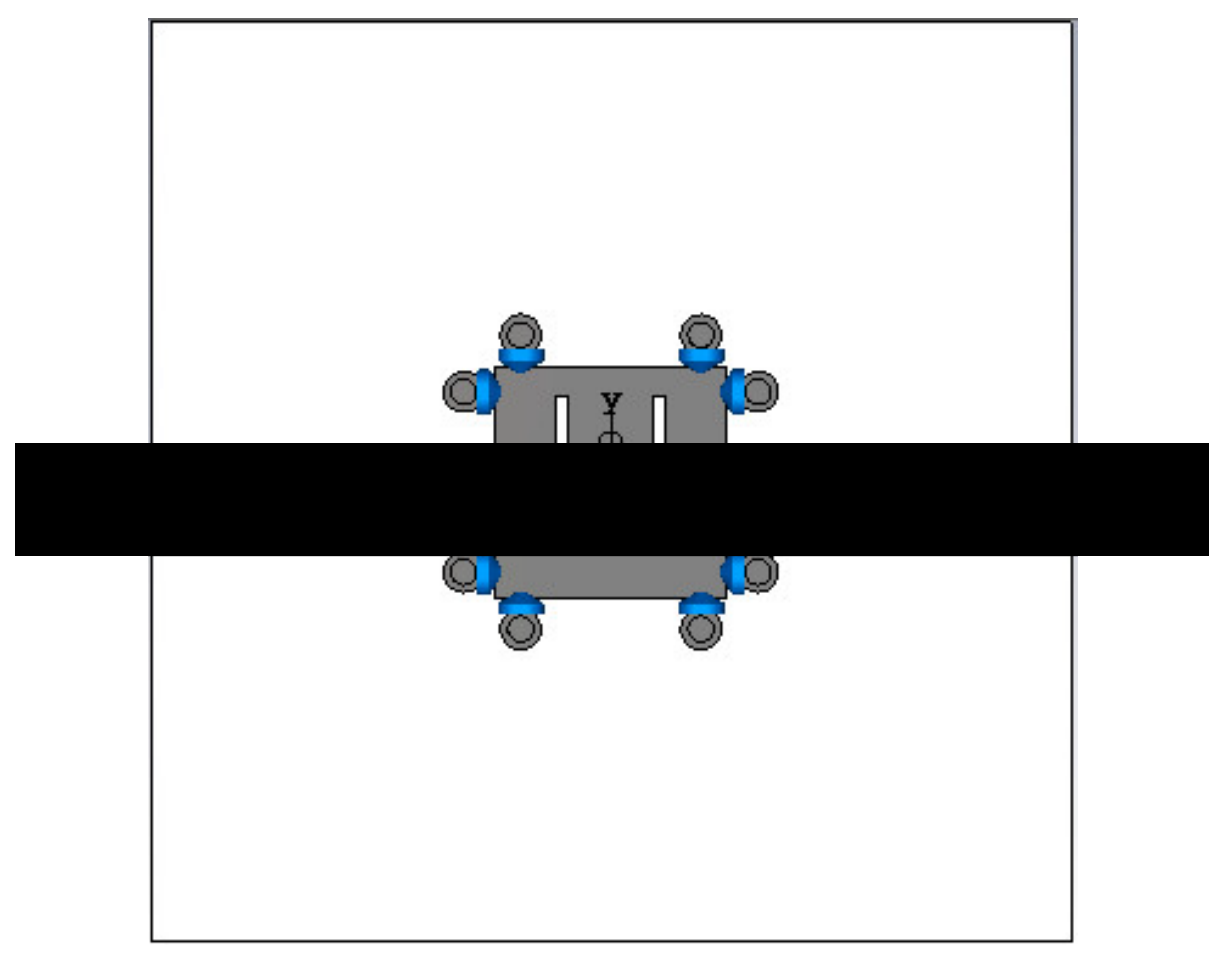

(a) Antenna design (Top view)

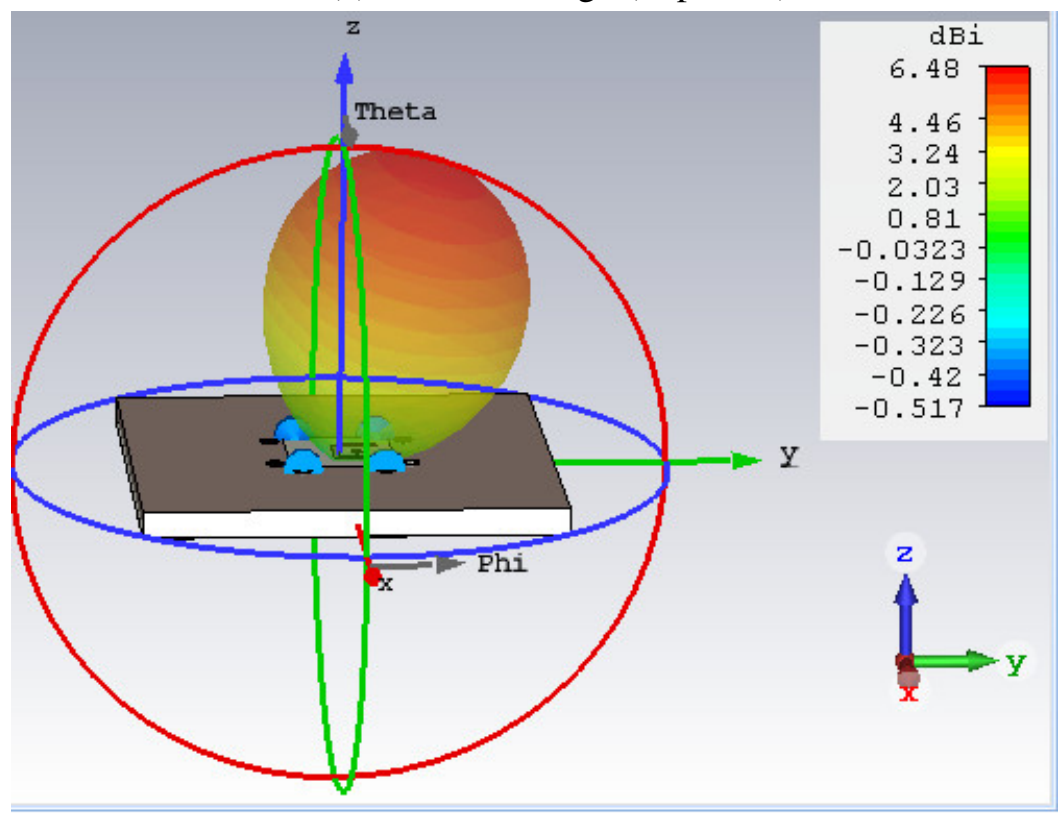

(b) Simulated farfield Directivity pattern

Fig. 3.4 U-slot reconfigurabe patch antenna by Qin et al. (2012) and simulated 3D Directivity pattern for configuration-I. 


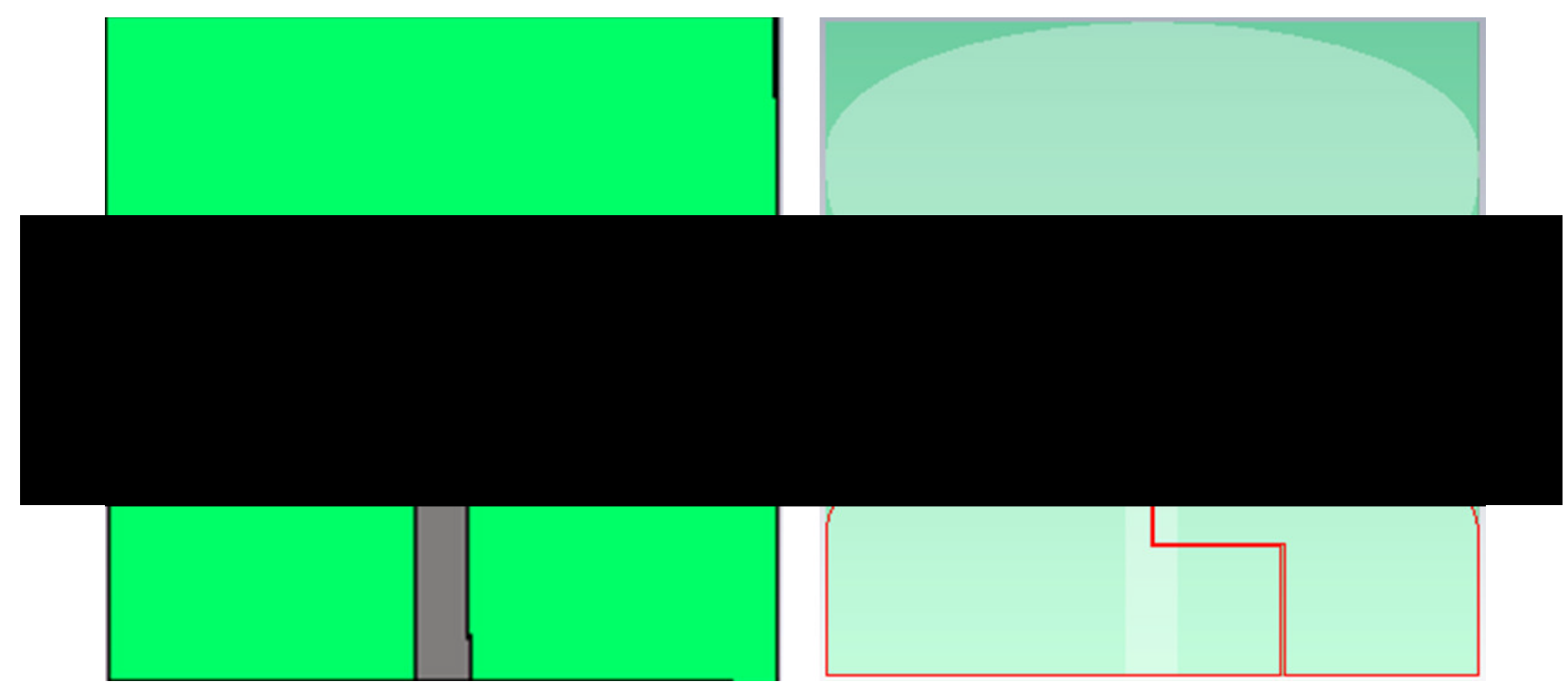

(a) Top view (Left) and Bottom view (Right).

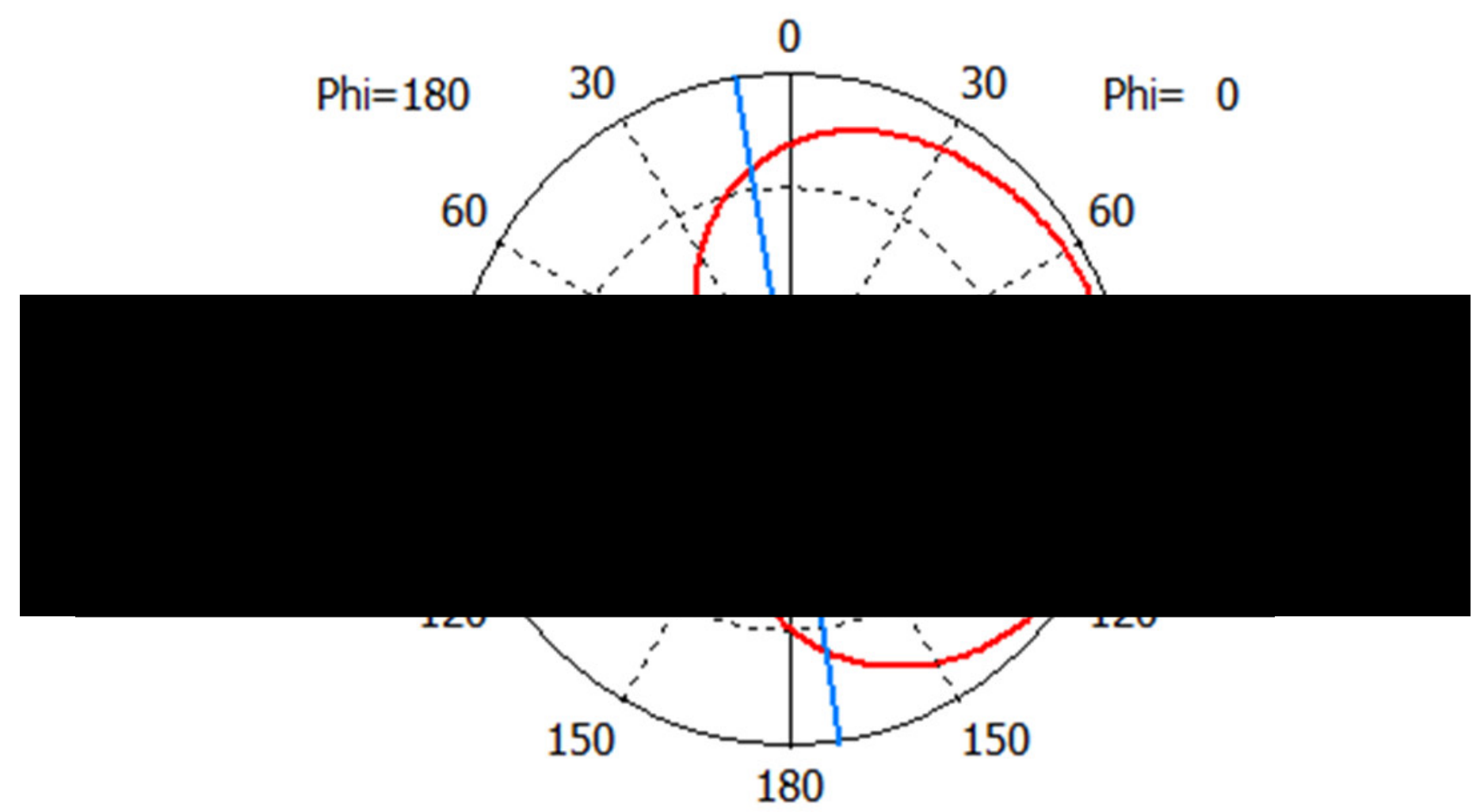

(b) Simulated polar plot of Directivity pattern at $\phi=-180^{\circ}$ for $5 \mathrm{GHz}$, for the ON-OFF state

Fig. 3.5 Pattern reconfigurable antenna design and farfield Directivity pattern by Li et al. (2011b). 
The planar elliptical topped monopolar antenna driven by a co-planar waveguide (CPW) reported in Li et al. (2011b) also uses 2 diodes and 3 configurations (OFF-OFF, OFF-ON and ON-OFF). It has a wide impedance bandwidth from $4 \mathrm{GHz}-6.5 \mathrm{GHz}$. This antenna design, is shown in Fig. 3.5. It is fed via a CPW on the top surface which is connected using 5 vias to a slotline feedline which in turn feed the elliptical monopolar antenna at the bottom surface of the substrate. Fig. 3.5 shows the farfield Directivity pattern at $5 \mathrm{GHz}$, when the Left diode is forward biased (ON) and the Right diode is reverse biased (OFF). During this ON-OFF configuration (i.e Right Slot feeding), the antenna generates a directive pattern pointed along the $+X$ axis. Similarly, it will generate a directive pattern pointed along the $-X$ axis when the two diodes are in OFF-ON (i.e Left Slot feeding) state. When in OFF-OFF state, the antenna will generate a nearly omni-directinal pattern as mentioned in Li et al. (2011b). Hence, this antenna has 3 reconfigurable beam patterns resulting in spatial diversity along the $+\mathrm{X}$ axis, -X axis and omni-direction.

The $2.3 \mathrm{GHz}$ circular patch pattern reconfigurable antenna reported in Lim and Lim (2013) also uses two p-i-n diodes to create two pattern combinations depending on the state of the diodes (ON-ON and OFF-OFF). It creates a monopole-like pattern in the OFF-OFF state and a directive pattern in the boresight direction (Z-axis). This method is similar to the planar circular, pattern reconfigurable antenna reported by Aboufoul et al. (2013). It uses a similar antenna design which is fed using a CPW. However, this antenna uses 4 p-i-n diodes instead of 2. It has an UWB frequency range from $2 \mathrm{GHz}$ to $10 \mathrm{GHz}$. It also has 3 operating modes with respect to the biasing of the 4 p-i-n diodes: OFF-OFF-OFF-OFF, OFF-OFF-ON-ON and ON-ON-OFF-OFF. The 3 modes generate a near omnidirectional pattern, a directional pattern along the $-\mathrm{X}$ axis and a directional pattern along the $+\mathrm{X}$ axis.

Nair and Ammann (2010) has reported a reconfigurable planar square patch antenna with resonant frequency at $2.1 \mathrm{GHz}$. It has an inner square patch $(8 \mathrm{~mm} \times 8 \mathrm{~mm})$ surrounded by a $5 \mathrm{~mm}$ thick square shaped ring with a $2 \mathrm{~mm}$ gap between the two. Two p-i-n diodes connect the ring to the square patch across this slot. This ring is then surrounded by a much wider outer square ring. Once again, the inner ring is connected to the outer ring via another two p-i-n diodes. This antenna design is shown in Fig. 3.6. When the inner diode along the -Y axis (D1) is forward biased, it creates an electrical path between the square patch and the inner ring. This results in a highly directive $(4.58 \mathrm{~dB})$ tilted beam $\left(\theta=25^{\circ}\right)$ pattern at $\phi=-90^{\circ}$. Additionally, when the outer diode along the $-\mathrm{Y}$ axis is also forward biased, it creates an electrical path between the outer ring, inner ring and the square patch. It results in a directive tiled beam at $\phi=+90^{\circ}$. Similarly when the other two diodes along the $-\mathrm{X}$ axis are forward biased in turn, while keeping the two diodes along the $-\mathrm{Y}$ axis in OFF state (i.e reverse biased), it generates two tilted beams in the direction of $\phi=180^{\circ}$ and 


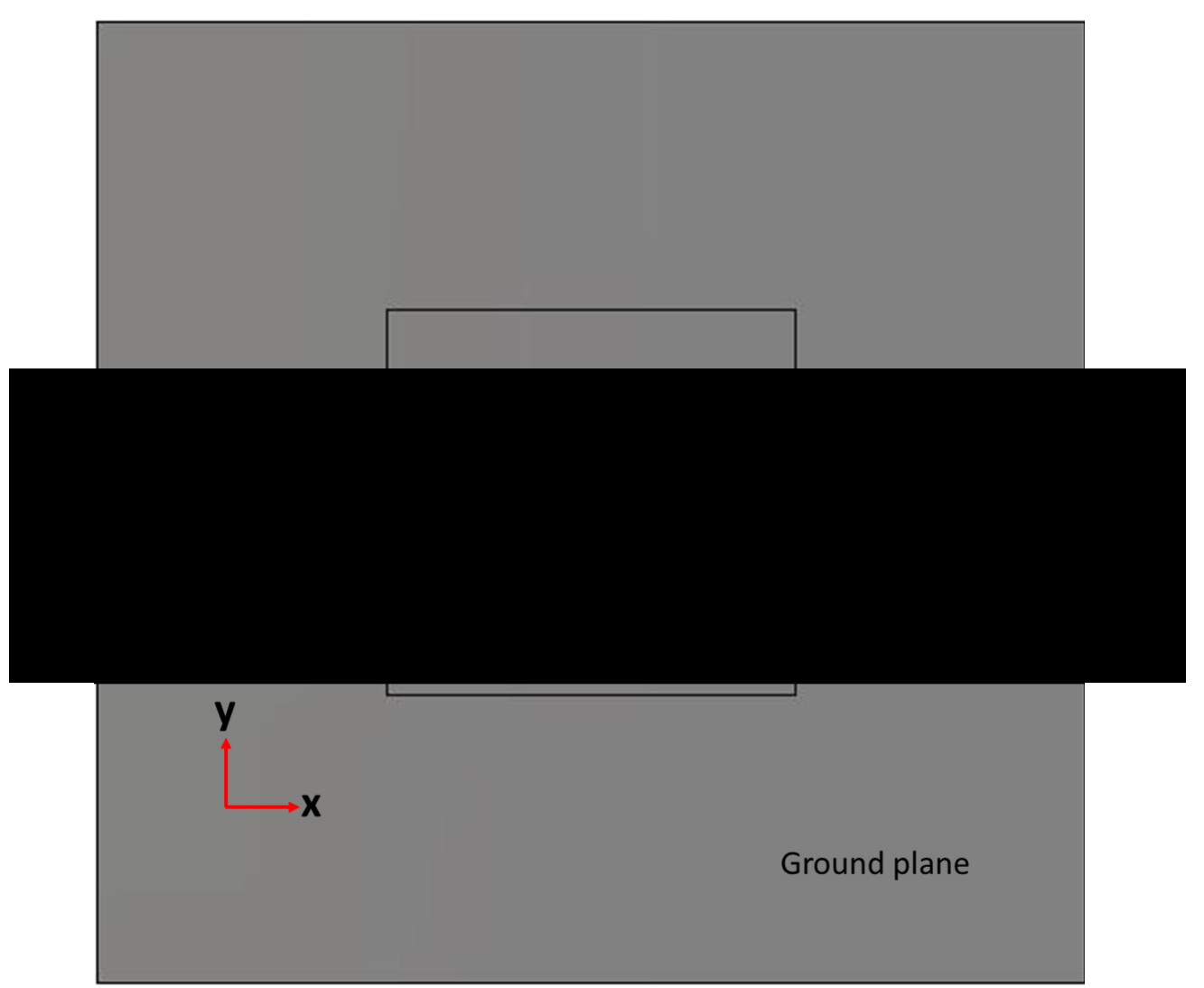

(a) Top view.

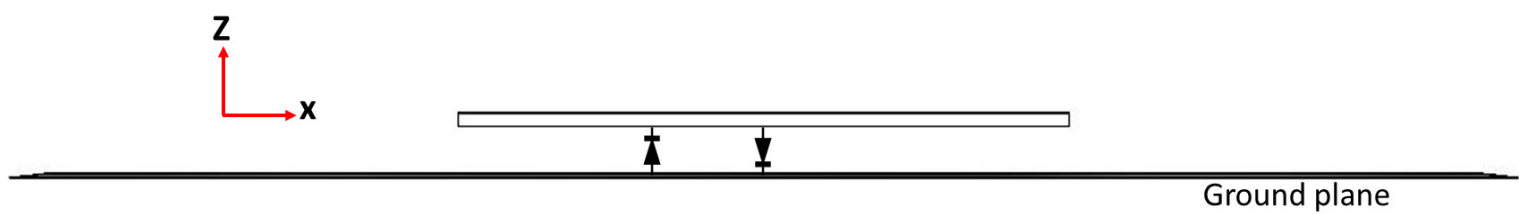

(b) Side view.

Fig. 3.6 Pattern reconfigurable antenna design by Nair and Ammann (2010). 
$\phi=0^{\circ}$ respectively. This antenna demonstrate beam switching in the 4 directions. However, the antenna beam patterns Directivity for the four cases is not uniform as it drops from a maximum of $4.58 \mathrm{~dB}\left(\phi=-90^{\circ}\right)$ to a minimum of $1.11 \mathrm{~dB}\left(\phi=180^{\circ}\right)$.

The pattern reconfigurable planar patch antenna reported by Han and Huang (2010) also uses $4 \mathrm{p}$-i-n diodes for beam reconfiguration. The 4 diodes surrounding a central rectangular patch $(18 \mathrm{~mm} \times 14.5 \mathrm{~mm})$ connect each side of the square patch to 4 smaller rectangular patches of varying sizes, around it. By forward biasing different combinations of the $4 \mathrm{p}$-i-n diodes, it electrically connects the central patch to different combinations of the outer patches. Therefore, by varying the area of the radiating surface of the antenna, it generates different beam patterns from $1820 \mathrm{MHz}$ to $2480 \mathrm{MHz}$. The Gain of the beam patterns reported, increases from $2 \mathrm{dBi}$ at $1820 \mathrm{MHz}$ to around $3 \mathrm{dBi}$ at $2480 \mathrm{MHz}$. Similar to the antenna reported by Nair and Ammann (2010), this antenna also fails to retain a consistently high directive pattern for all the switching states.

Patron et al. (2013) also presents a pattern reconfigurable antenna resonating at $3.8 \mathrm{GHz}$ which uses 4 p-i-n diodes for 5 pattern configurations. When all 4 diodes are forward biased, this antenna behaves like an Alford loop antenna and it produces a near-omnidirectional pattern which is horizontally polarized. When each of the four arms of the antenna is connected to the central feed by biasing the respective diode, the other 3 arms of the antenna act as reflectors when the other $3 \mathrm{p}$-i-n diodes are reverse biased. Therefore it produces a directional beam pointing in the direction of the excited arm. Hence, this antenna also produces 4 directional beams in the $-\mathrm{X},+\mathrm{X},-\mathrm{Y},+\mathrm{Y}$ axial directions a well as an omnidirectional pattern.

Chen et al. (2012) presents a dual band notched slot antenna shown in Fig. 3.7 (Left). It uses 2 p-i-n diode switches which have 4 states (OFF-OFF, OFF-ON, ON-OFF, ON-ON). By controlling the switching of the p-i-n diodes $S_{1}$ and $S_{2}$, the total length of the inverted $\mathrm{C}$-shape notches can be adjusted in order for their lengths to be roughly $\lambda / 2$ at a particular frequency. This relationship between the length of the notch, $L_{s l o t}$, and the frequency $f_{\text {notch }}$, as mentioned by Chen et al. (2012) is shown below.

$$
f_{\text {notch }}=\frac{c}{2 L_{\text {slot }} \sqrt{\varepsilon_{\text {eff }}}}
$$

where $\varepsilon_{\text {eff }}$ is given by

$$
\varepsilon_{\text {eff }}=\frac{1+\varepsilon_{r}}{2}
$$

When each switch is activated (i.e switched $\mathrm{ON}$ ), the notches will create an impedance mismatches at the respective frequency band $(3.5 \mathrm{GHz}$ or $5.5 \mathrm{GHz})$, thereby resulting in a high VSWR not suitable for efficient transmission. This antenna has a high bandwidth of 3.1 


\section{Literature review}

$\mathrm{GHz}$ to $12.5 \mathrm{GHz}$. However, its reported $E_{\theta}$ and $E_{\phi}$ Gain patterns are nearly monopole-like or omni-directional respectively with very low Gain.

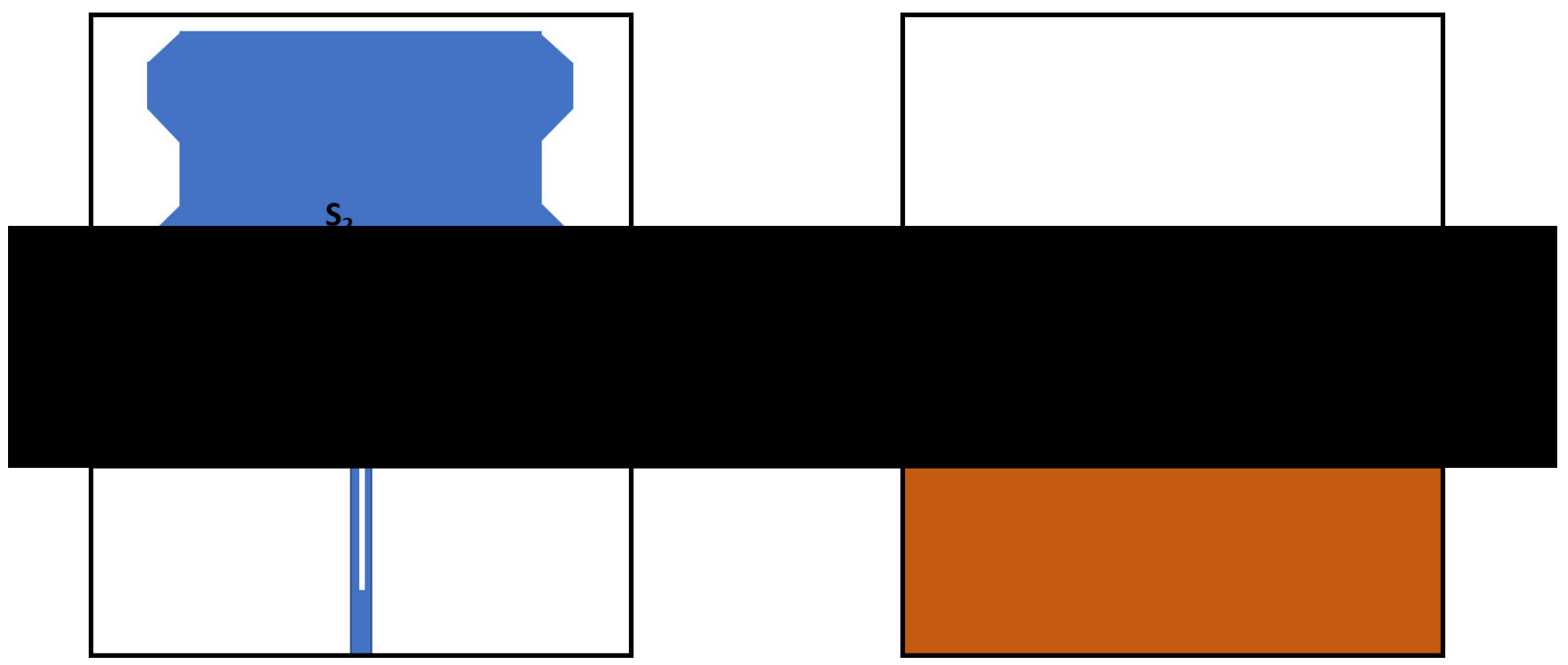

Fig. 3.7 Reconfigurable UWB notch antenna design by Chen et al. (2012). Top view (Left) and bottom view showing ground plane (Right).

Radio frequency microelectromechanical system (RF MEMS) switches are also another popular way of switching between antenna radiating elements to reconfigure antenna radiation patterns. By using RF MEMS switches, the different radiating elements of the antenna can be fed separately. RF MEMS switches can be monolithically integrated as a single integrated chip, which is an advantage over using separate $\mathrm{p}-\mathrm{i}$-n diodes.

The antenna presented by Cao et al. (2012) has an embedded planar feeding network at the bottom layer of the substrate, which uses a Wilkinson power divider. The RF input feed of the antenna is divided into 4 branches which in turn, feeds the 4 rectangular patches on the top as shown in Fig. 3.8. Using one group of RF MEMS switches (i.e either Switch group A or Switch group B) $R F_{I N}$ can be switched to the 4 ports (Port 1 to 4 ) using the power divider network as shown in Fig. 3.8 (Right). For example, when Switch group B is ON, The 4 ports will have phase shifts of $\phi=0^{\circ}, \phi=90^{\circ}, \phi=180^{\circ}$ and $\phi=270^{\circ}$ respectively. This results in a broadside directive pattern in the direction of $\phi=0^{\circ}$. Switching ON Switch group A, while keeping Switch group B will result in all ports being in-phase and hence, the antenna will generate a pattern similar to that of a short monopole antenna. This antenna resonates at the frequency of $2.1 \mathrm{GHz}$. It uses $8 \mathrm{RF}$ MEMS switches to generate 2 reconfigurable pattern combinations and has a complex feeding structure. Zohur et al. (2013) also reports a pattern reconfigurable antenna which has two switchable patterns activated using a single RF MEMS switch. It has an inverted T-shaped radiating element and a meander line shaped radiating 


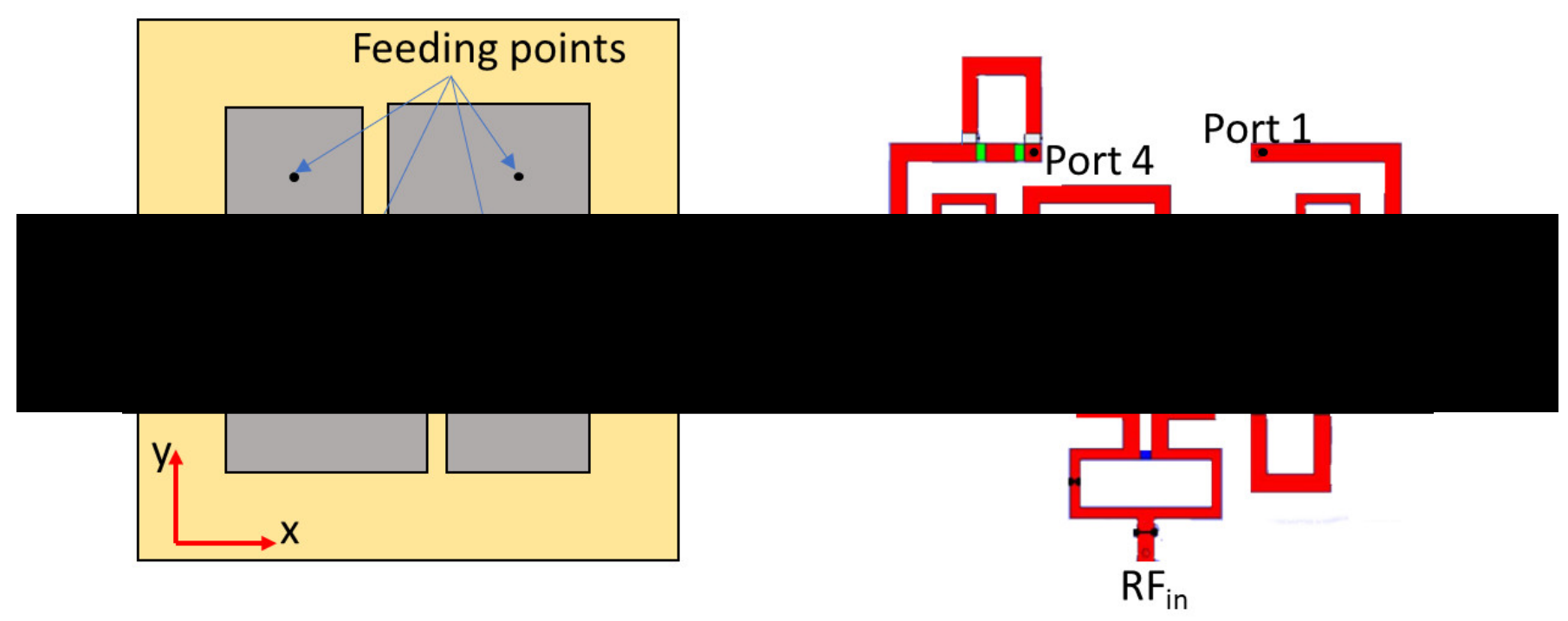

Switch A

Switch B

Fig. 3.8 Top view of 4-feed reconfigurable 4-patch antenna (Left) by Cao et al. (2012) and its feeding network incorporating RF MEMS switches (Right).

element separated by the RF MEMS switch. The ON/OFF state of the switch which allows the current to travel along the T-shaped radiating element or the meander line section, will alter the electrical length the current travels. Therefore, this antenna generates two beam patterns, one at $710 \mathrm{MHz}$ (for T-mode) and the other at $4.96 \mathrm{GHz}$ (for meander line mode). The two patterns thus generated are not in the same frequency band.

Kim and Rahmat-Samii (2011) reports a slot loaded patch antenna fed with a complex bias network. The patch antenna is of dimension $17.7 \mathrm{~mm} \times 20.5 \mathrm{~mm}$ and its slot is 19.6 $\mathrm{mm} \times 2.2 \mathrm{~mm}$. The bias network consists of a $90 \mathrm{~V} \mathrm{DC}$ bias line with a spiral inductor filter on either side followed by an interdigital gap capacitor filer. This capacitor is based on the principles mentioned by Alley (1970). It is connected to a RF MEMS switch whose ON/OFF state will connect/disconnect it to the slot loaded patch antenna, thereby giving rise to two reconfigurable radiation patterns at $4.88 \mathrm{GHz}$ and $4.57 \mathrm{GHz}$ respectively. The two radiation patterns have high directivity of $7.1 \mathrm{~dB}$ and $7.6 \mathrm{~dB}$ at the broadside $\left(\phi=90^{\circ}\right)$. Therefore, while this antenna has a high directive pattern, they are in the same broadside direction and hence, give only polarization diversity and frequency diversity and it does not give spatial diversity. It also uses a complex bias network which is difficult to implement.

The pixeled reconfigurable PCB patch antenna by Besoli and Flaviis (2011) also uses RF MEMS to connect adjacent patches to create a reconfigurable beam pattern. This antenna has an array of square patches, each of which can be connected to others around it by switching 
ON an RF MEMS switch. By switching ON or OFF the RF MEMS switches, it is possible to create a reconfigurable aperture size for the radiating antenna. Therefore, it achieves pattern as well as frequency diversity based on the states of the multiple RF MEMS switches. This type of antenna requires a large number of RF switches and a complex switching circuitry. Rodrigo and Jofre (2012) has also reported using a similar technique in its multi-sized pixel antenna which uses p-i-n diodes instead of RF switches to short the patches together. However, this antenna uses different sized pixels to create different pattern combinations such as planar monopole, printed dipole, printed patch etc.

The double square loop antenna design reported by Kim et al. (2011a,b) consists of two concentric microstrip square loop connected by two switches along the $-Y$ and $+Y$ axis. The inner square loop has side of lengths of $\lambda$, while the outer square loop has sides of $2 \lambda$ at 12.8 GHz. The inner square loop is fed at the center of one of its microstrip arms. The two switches will have 3 configurations: OFF-ON, ON-OFF, ON-ON. The first two configuration modes will generate a directive tilted $\left(\theta=40^{\circ}\right)$ beams at $\phi=230^{\circ}$ and $\phi=130^{\circ}$ respectively while the third mode $(\mathrm{ON}-\mathrm{ON})$ will generate an axial beam along the $\mathrm{Z}$-axis. The three beam patterns have directivity of $5.83 \mathrm{~dB}, 5.78 \mathrm{~dB}$ and $5.32 \mathrm{~dB}$ respectively. The resonant frequencies for the 3 modes are in the same frequency band $(14.5 \mathrm{GHz})$ as well. While it is possible to connect the inner square loop to the outer loop via two further RF switches along the $-\mathrm{X}$ and $+\mathrm{X}$ axis, this aspect has not been explored nor reported.

Bahng et al. (2009) has reported a simple folded dipole beam reconfigurable antenna for $2.45 \mathrm{GHz}$. It consists of two rectangular loops or folded dipoles joint together by a common side. The antenna is fed at the center of this common side. Two pairs of switches at the end of the common side, connect each loop (folded dipole) to this common side with the central feeding. When the pair of switches on one side is ON and the other pair is OFF, it completes the rectangular loop for one side while leaving the loop on the other side open. The open loop will act as a reflector to the loop that is closed, through which the current flows. The reflected power from the reflector will create a slightly directive $(2.29 \mathrm{~dB})$ doughnut shaped pattern, with the maximum Gain in the boresight direction, away from the reflector. This antenna has symmetry and hence, will create two similar patterns in the boresight direction $(+\mathrm{Y}$ and $-\mathrm{Y}$ axis). However, its reported simulated radiation patterns reported have low directivity.

A 6-beam pattern reconfigurable antenna has been reported by Cai et al. (2012) shown in Fig. 3.9a (Top) uses a single feed. Its ground plane consists of a circular patch and 3 rectangular patches attached to its perimeter, spaced equally at $120^{\circ}$ intervals. On the top side, there are 6 radiating elements (patches) which are connected to six switches. When each switch is activated while keeping the other 5 switches OFF, the generated beam pattern has around $5 \mathrm{dBi}$ Gain in the broadside direction as shown in Fig. 3.9b (Bottom). Due to the 
symmetry of this antenna, each of the Gain patterns generated by switching the six switches are similar. They are in the $\phi=30^{\circ}, \phi=90^{\circ}, \phi=150^{\circ}, \phi=210^{\circ}, \phi=270^{\circ}$ and $\phi=330^{\circ}$ directions. This antenna therefore can steer the beam in the azimuth direction with near $360^{\circ}$ coverage. It's generated patterns also stay consistently similar during beam reconfiguration.

Zhang et al. (2012) also has presented a wide-band pattern reconfigurable antenna with two sides that act alternatively as a reflector or a radiator. Its design is shown in Fig. 3.10. The antenna comprises of a CPW feeding line, a pair of step-shape patches printed side by side and two switches $\left(S_{1}\right.$ and $\left.S_{2}\right)$ that connect the CPW feedline to either side. By switching one switch ON and keeping the other OFF, the feedline can be connected to the patch on one side, making it act as the radiator. The reverse patch on the other side, which is identical to the radiator will act as a reflector, due to the switch connecting it to the CPW feedline being in OFF state. This type of simple configuration results in producing a switchable directive two-beam pattern. It is designed for $3.3 \mathrm{GHz}-6 \mathrm{GHz}$ frequency range. The beam steerability of this antenna is limited to switching its main beam direction from $\phi=0^{\circ}$ to $\phi=180^{\circ}$.

Deo et al. (2009a) has presented an 8 beam reconfigurable antenna design using an 8 feed, planar circular ring type antenna with a substrate backed by a ground plane. The circular ring antenna has 8 feed points, spaced equally along the ring by $45^{0}$ angle separations. The reported simulated work produces a sharp conical pattern $\left(E_{\theta}=12 \mathrm{dBi}\right.$ Gain $)$ tilted beam $\left(\theta_{\max }=45^{\circ}\right)$ covering a $45^{\circ}$ azimuth angle. By switching between the 8 feeding ports sequentially, the beam can be steered in the azimuth direction to cover the entire $0<\phi<360^{\circ}$ range. The reported simulated work shows a resonance at $4.35 \mathrm{GHz}$ with a $500 \mathrm{MHz}$ impedance bandwidth. This type of antenna, by virtue of having a sharp tilted conical pattern with $0<\phi<360^{\circ}$ beam steerability, is ideal for multipath mitigation, user tracking and beam steering applications. However, the reported work by Deo et al. (2009a) contains only simulated results based on an infinite ground plane and a substrate. It lacks validation by way of a prototype built. In addition, the beam switching mechanism is not reported in the published work.

RF MEMS switches are linear devices. They consume almost zero power, have high isolation and have very low insertion loss compared to $\mathrm{p}$-i-n diodes. They however, require a voltage up-conversion if used in a portable antenna transmitter system. They have lower reliability and need extra lumped components to implement, as mentioned by Aboufoul et al. (2012). On the other hand, GaAs switches will also have low insertion loss and have good isolation while consuming low power, similar to RF MEMS switches. But they have the added advantages of being smaller in size, having very good switching speed performance and having simplicity in design. 


\section{Literature review}

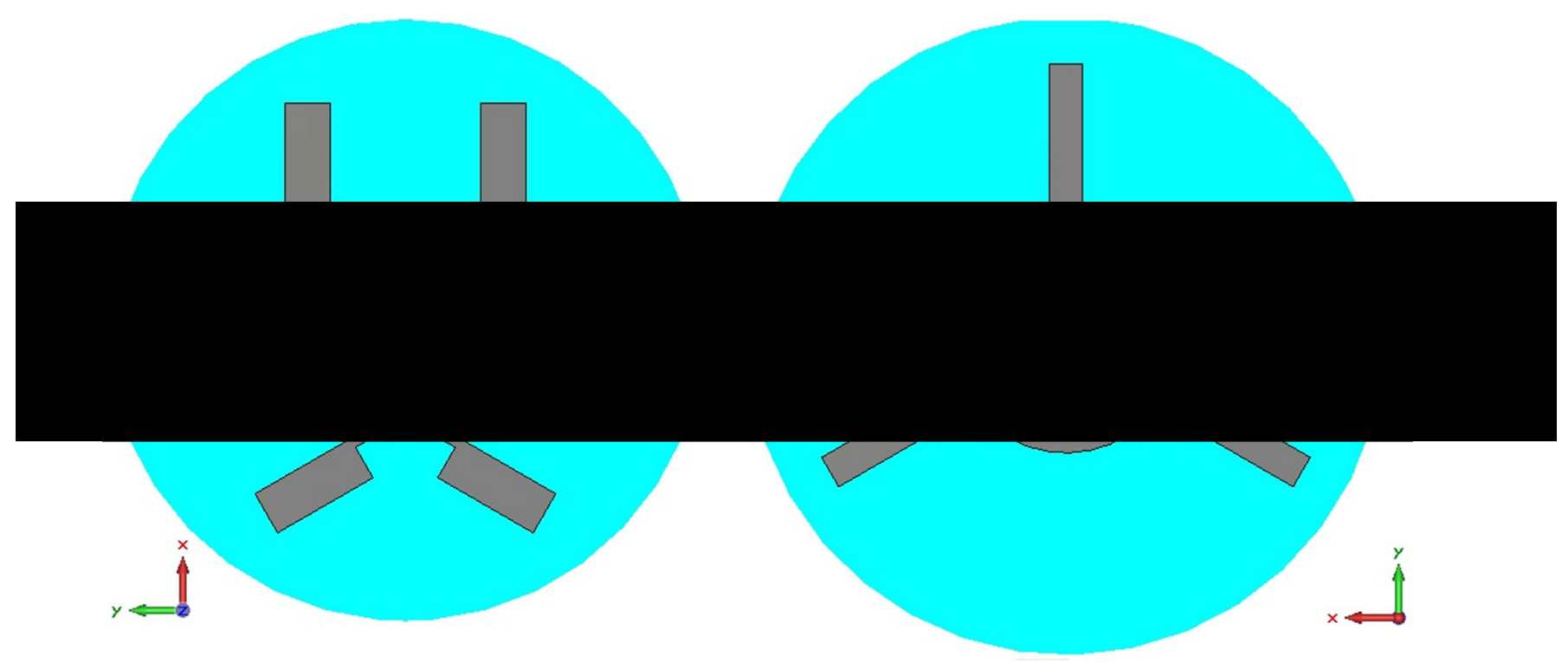

(a) Pattern reconfigurable antenna design by Cai et al. (2012). Top view (Left) and Bottom view with feeding port (Right).
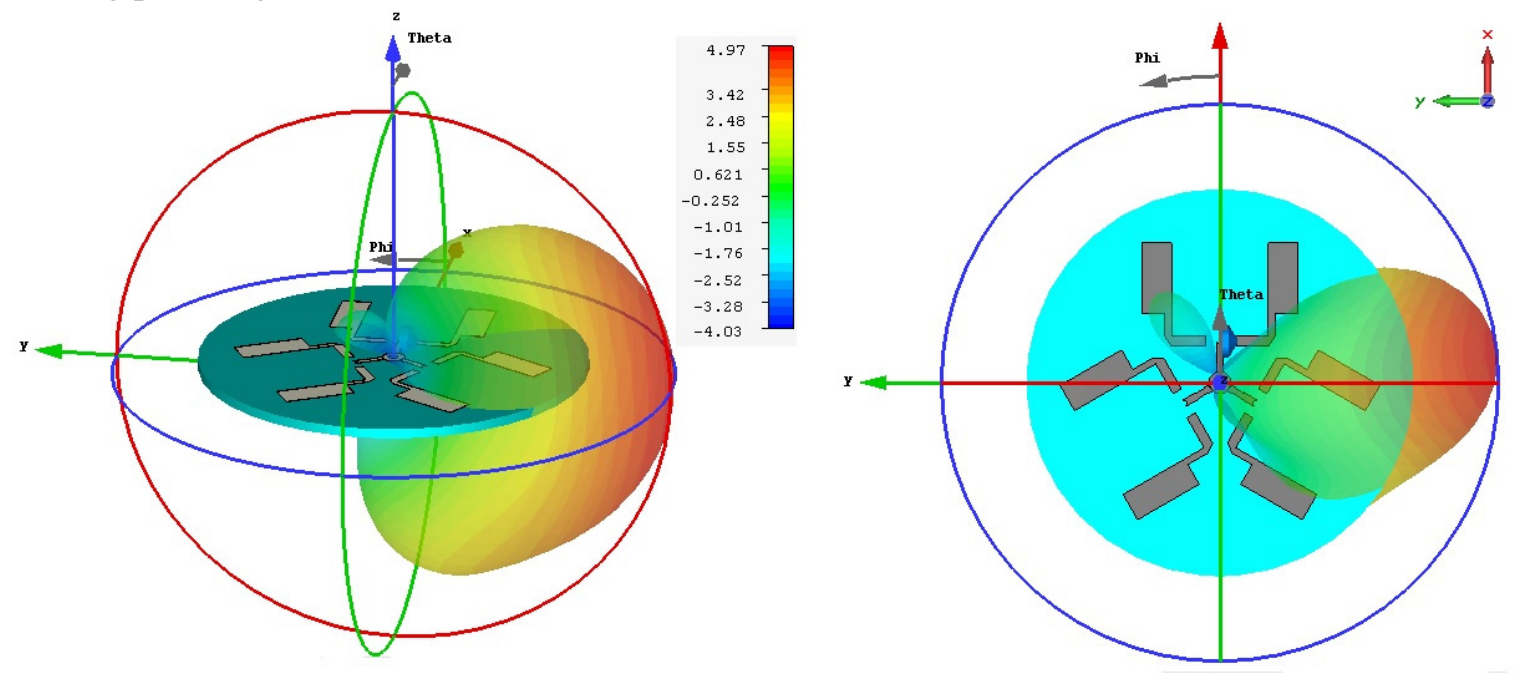

(b) The antenna Gain pattern showing from the perspective angle (Left) and Top view (Right). 


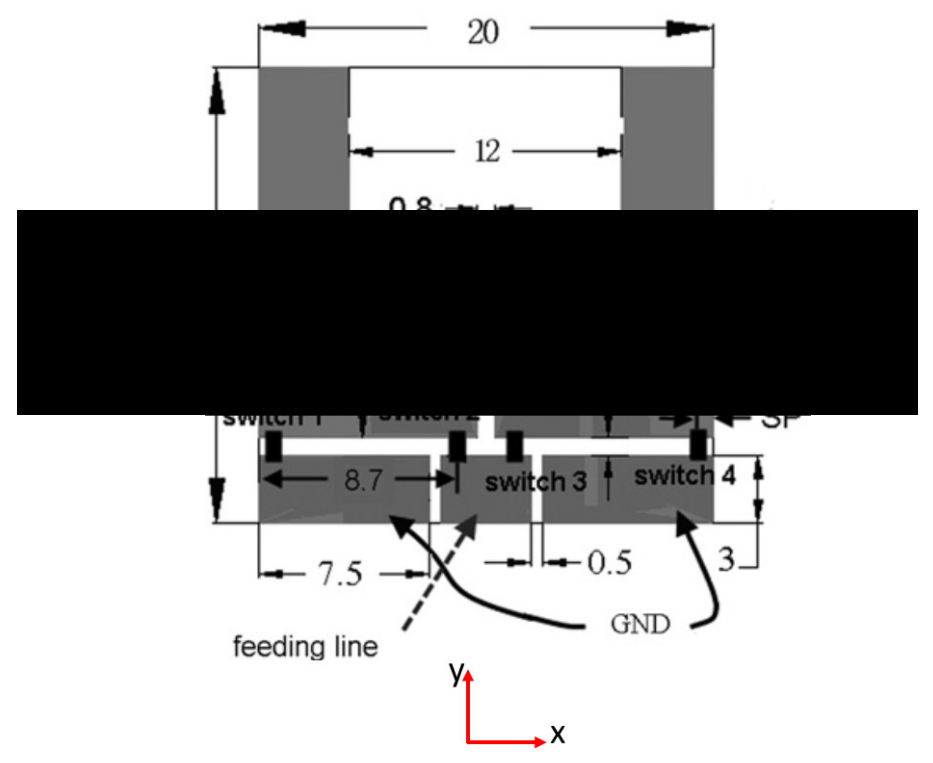

Fig. 3.10 Pattern reconfigurable antenna design with alternate reflector and radiator sides by Zhang et al. (2012).

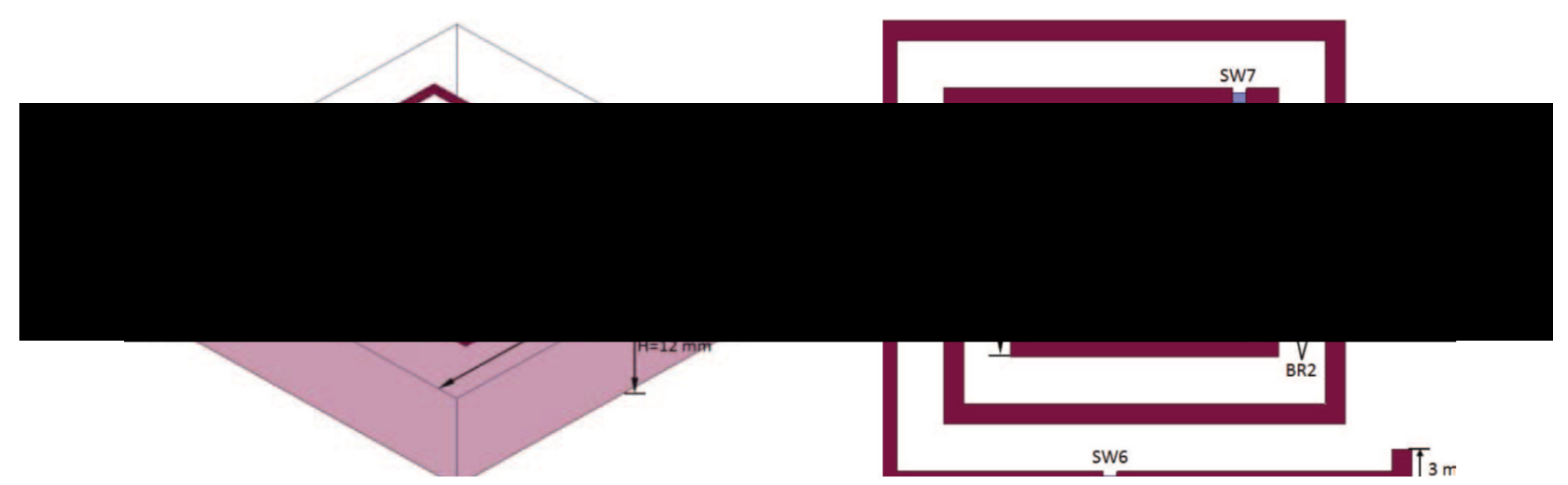

Fig. 3.11 Conformal pattern reconfigurable spiral antenna by Gong et al. (2013). 
The pattern reconfigurable antenna discussed in Gong et al. (2013) uses a single-poledouble-throw (SP2T) GaAs switch to control the beam switching of a UWB spiral patch antenna. This design which is shown in Fig. 3.11, is similar to the pattern reconfigurable spiral antenna presented by Jung et al. (2006) which uses RF MEMS switches or the reconfigurable spiral antenna presented by Mehta et al. (2006) which proposes to use either RF MEMS switches or $\mathrm{p}-\mathrm{i}-\mathrm{n}$ diodes (method not specified).

The simulated results presented by Gong et al. (2013) show a good impedance bandwidth from $2 \mathrm{GHz}$ - $10 \mathrm{GHz}$. By switching between stubs, on either side of the microstrip feed line, it reconfigures the tilted radiation pattern at different frequencies. Its reported Gain patterns in the azimuth plane $\left(E_{\phi}\right)$ retain a constant shape while the elevation plane patterns $\left(E_{\theta}\right)$ change resulting in a decrease in its Directivity during the beam switching operation.

A p-i-n diode or an RF switch can be used to switch ON or OFF radiating elements in an antenna in order to reconfigure its beam pattern. Once the ON/OFF state is configured, the radiation pattern of the antenna stays fixed until the switch status is changed again. In the single unit pattern reconfigurable antennas discussed up to now, the radiation patterns have a fixed number of pattern combinations due to the diodes or the switches used. The use of varactor diodes on the other hand, provide the ability to continuously tune the antenna's radiation pattern. Hence, antennas using varactor diodes have a larger range of pattern reconfigurability.

Zhang et al. (2013) presents an electronically steerable pattern reconfigurable antenna on a frequency selective surface (FSS) which uses varactor diodes for tuning. This antenna design is shown in Fig. 3.12. It consists of a monopole feed antenna at the center of the system shown in the figure. It provides an omni-directional pattern. An active frequency selective surface (AFSS) is made up using 10 columns of unit cells arranged in a cylindrical shell, as shown in Fig. 3.13. Each column has 17 unit cells. By tuning the reverse biased varactor diodes, the path of the EM wave emitted from the inner antenna can be altered, thereby making the antenna beam steerable. The beam steering can be achieved by tuning whole columns in the AFSS into either reflective mode, transparent mode or partial transparent mode. EM energy is blocked from the unit cell columns in reflective mode and they pass through the transparent unit cell columns. By varying the ratio of reflective mode columns to partial reflective columns to the transparent columns, the beam's directivity and direction can be continuously altered. This type of design, although achieving $360^{\circ}$ beam steering capability, is not very practical or portable due to its bigger size. It also requires a complex wiring control circuit to control the beam steering.

The antenna design presented in Jiang et al. (2012) also makes use of tunable varactor diodes for pattern reconfigurability. It has a planar square slot antenna on a sapphire substrate 


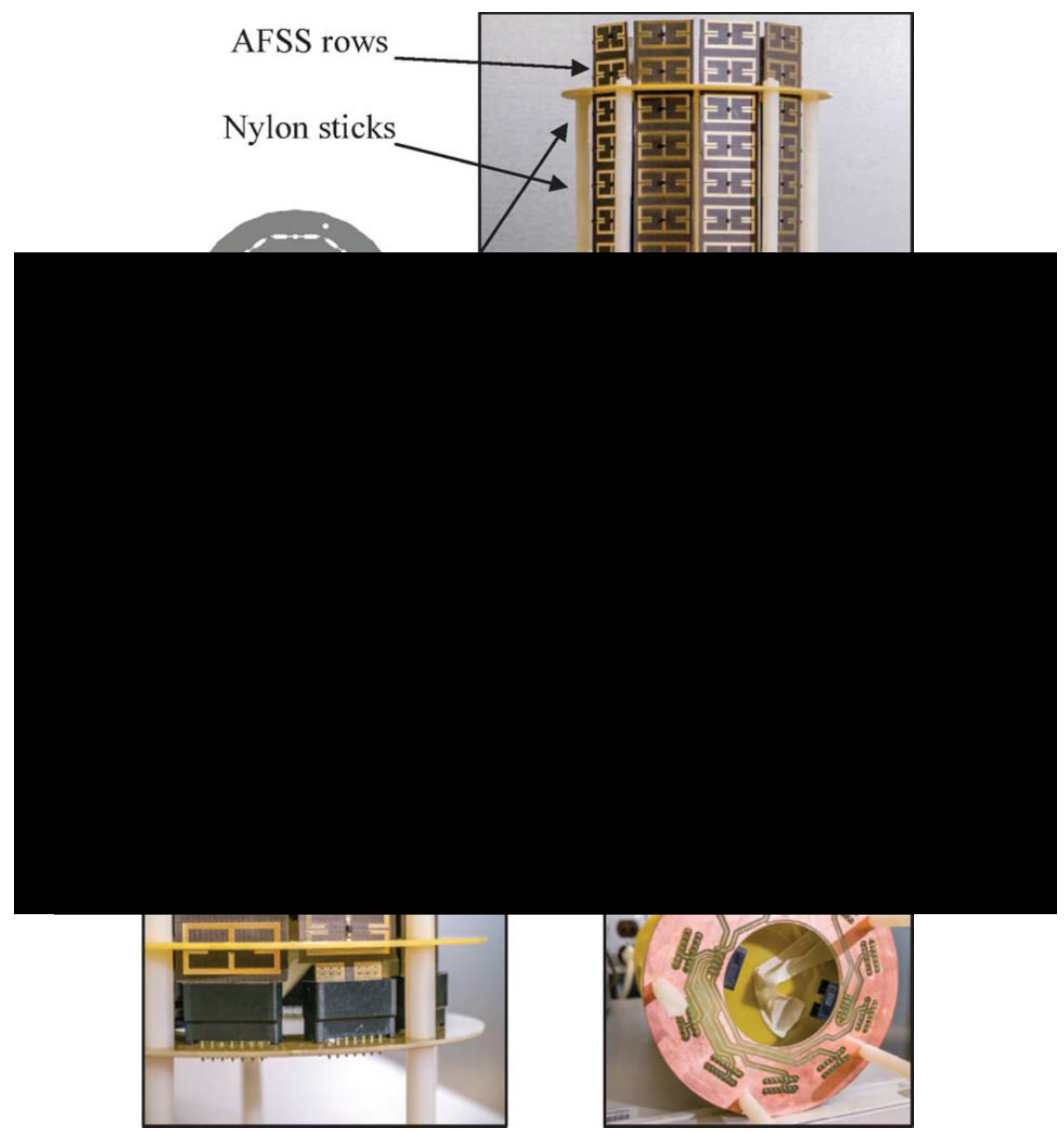

Fig. 3.12 The frequency selective reconfigurable antenna structure by Zhang et al. (2013). 


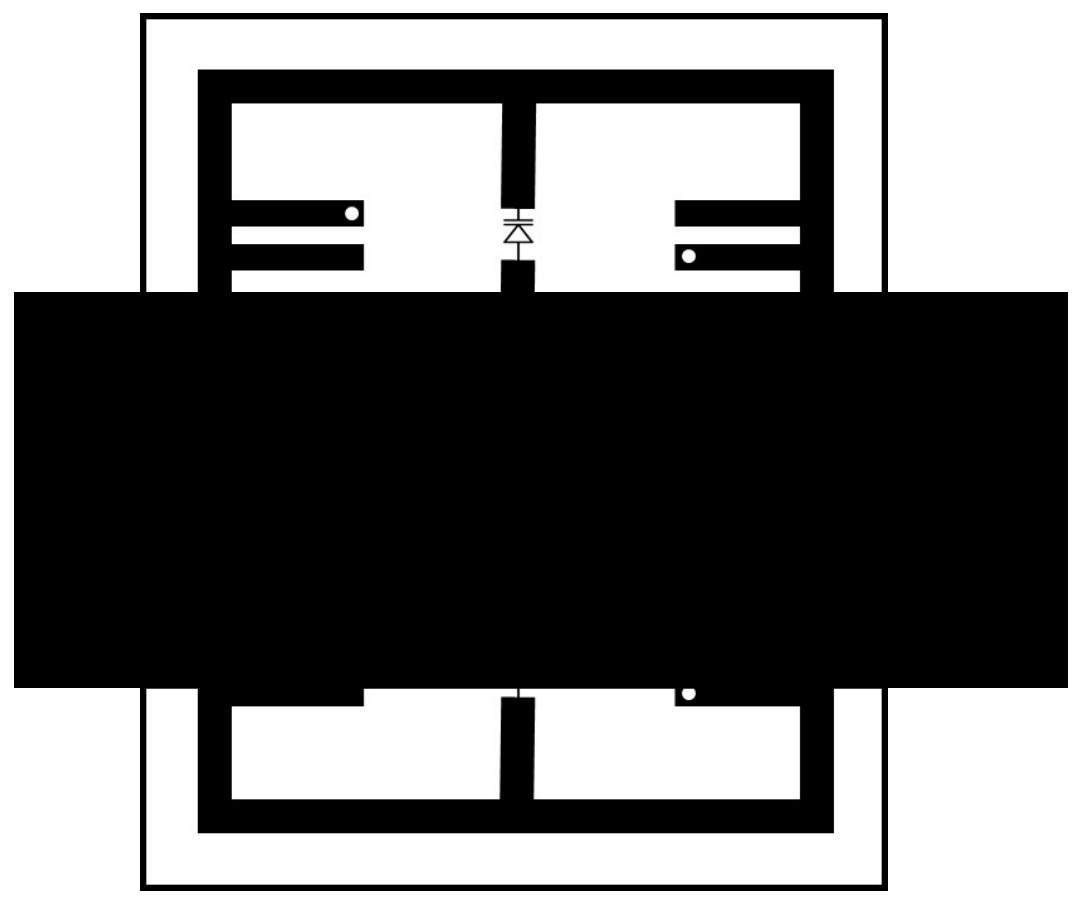

Fig. 3.13 Unit cell containing two reflectors with varactor diodes as shown in Zhang et al. (2013).

$\left(\varepsilon_{r}=9.7\right)$ which is fed by a CPW feedline. It uses Ferroelectric (FE) Barium Strontium Titanate (BST) thin film varactors to load the CPW to the square slot antenna. By varying the tunable capacitance of the FEBST varactors, the antenna pattern is reconfigured for different frequencies. Jiang et al. (2012) reports that when the FE BST varactors were used for frequency tuning, the antenna's maximum normalised measured gain dropped by around $3 \mathrm{dBi}$. Therefore, this type of method is not suitable for antennas with low gain patterns due to the effect that FE BST has on the antenna's gain.

\subsection{Pattern reconfigurable antennas using Frequency Se- lective Surfaces}

Similar to the antenna presented in Zhang et al. (2013) which uses an AFSS, several other pattern reconfigurable antennas using FSS have also been presented in literature. FSS are surfaces with periodic elements which act as spatial or frequency filters. When these frequency filters are tunable, the surface become a FSS. When an antenna is mounted on a FSS or is surrounded by it, as in the case with the antenna presented in Zhang et al. (2013), the FSS will allow EM energy of certain frequencies to pass through and block EM energy at certain frequencies. A FSS will act to block EM waves, in the same way that continuous 
strips create a stop-band for the incident EM waves polarized along the strips (Jazi and Denidni, 2010a). The unit cells in the FSS will act as either a reflector when active or an absorber when deactivated. When some unit cells in the FSS act as a reflector while the rest are deactivated, it will alter the antenna's beam direction and pattern. Thus, an antenna that uses a tunable FSS will have a reconfigurable Gain pattern which is also frequency dependent.

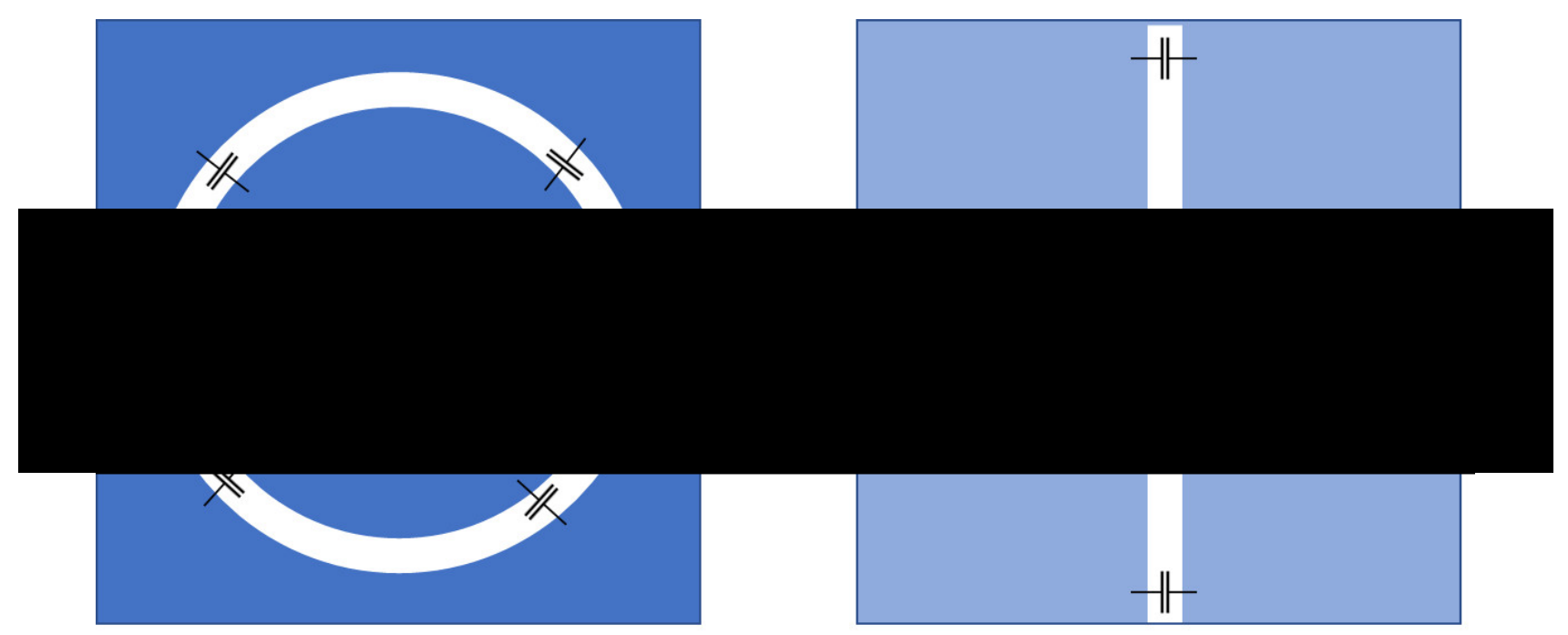

Fig. 3.14 Top view (Left) and bottom view (Right) of the unit cell design of FSS proposed by Yuan et al. (2013).

The FSS proposed by Yuan et al. (2013) comprise of a surface with unit cells whose design is shown in Fig. 3.14. Two groups of 4 Capacitors each, $C_{1}$ and $C_{2}$ are connected to the top surface (Fig. 3.14 (Left)) and the bottom surface (Fig. 3.14 (Right)) respectively, as shown. By tuning the capacitance of $C_{1}$ while keeping the group $C_{2}$ constant and by tuning the capacitance of $C_{2}$ while keeping capacitance of $C_{1}$ constant, the band gap frequencies of the FSS can be altered.

Jazi and Denidni (2010a) also proposes a reconfigurable antenna using an active cylindrical frequency selective surface (ACFSS). This antenna's FSS structure design is similar to Zhang et al. (2013) where the ACFSS is arranged in the form of a cylinder. Its ACFSS contains unit elements made up of simple strip dipoles which are connected using p-i-n diodes in a mesh like structure. It has 10 columns of unit cells surrounding a dipole antenna along its cylindrical axis. At the resonant frequency of the strip dipoles which make up each unit element, the ACFSS acts as a EM opaque barrier. By switching the p-i-n diodes ON/OFF the active area of the FSS can be shifted and hence the frequency stop band can also be shifted. When half the columns of unit cells are active, the Gain pattern created by the dipole antenna in the center becomes directive because one side of the cylinder blocks the 
EM waves and the opposite side becomes transparent to EM wave propagation. This creates a directive pattern for the antenna located at the center. Therefore, this antenna is a pattern reconfigurable and frequency reconfigurable antenna structure, whose beam can be steered in a near $360^{\circ}$ azimuth direction by use of the p-i-n diode controlled AFSS.

The antenna presented in Niroo-Jazi and Denidni (2013) also uses a similar cylindrical shaped FSS as in Jazi and Denidni (2010a) and Yuan et al. (2013). However, it uses 25\% less active unit cells and aperture size. The FSS is made up of one semi-cylinder (one half side of a cylinder) which is partially-reflective due to its design and the other half of the cylinder transparent to EM waves at the particular frequency. The half of the cylindrical FSS which is transparent to EM waves, consist of 5 columns of unit cells which can be controlled using p-i-n diodes. By controlling the biasing of these 5 columns in a sequential manner, the directive beam can be steered in the azimuth plane in a range of roughly $0<\phi<180^{\circ}$. Similar cylindrical shaped FSS have also been proposed by Edalati and Denidni (2011); Jazi and Denidni (2010b); Wang et al. (2012).

The reconfigurable antenna design, employing a FSS proposed by Edalati and Denidni (2013) has a cylindrical FSS surrounding an antenna aligned along its center line. It has vertical metallic plates dividing the cylinder into 6 equal sectors. Two metal conical shapes have been added to the top and bottom of the cylinder as shown in Fig. 3.15 in order to make the beam more directive. By switching OFF the diodes in the unit cells in one sector at a time, while keeping the rest of the 5 sectors in ON state, it is able to steer the beam in $360^{\circ}$ azimuth range, covering one $60^{\circ}$ sector at a time.

Ko et al. (2013) has proposed a cubic structure for the dual band pattern reconfigurable antenna using FSS. It has a dual band feeding antenna placed at the center, surrounded by 4 walls with dual band frequency selective reflectors. Each frequency selective reflector has two groups of rectangular loops which are controlled by 4 switches. The dual band feeding antenna resonates at $2.55 \mathrm{GHz}$ and $5.25 \mathrm{GHz}$ respectively. Its beam pattern is directive (3.22 $\mathrm{dB}$ at $2.45 \mathrm{GHz}$ and $7.53 \mathrm{~dB}$ at $5.25 \mathrm{GHz}$ ). However, it also has a strong side lobe in the reverse direction ( $\phi=-180^{\circ}$ from main beam direction) as well.

All the antennas that use FSS that were discussed in this section have cylindrical shapes and they have directive beam patterns. However, they have complex electronic control networks for the unit cells in the FSS, are bulky and hence, are not portable structures. 


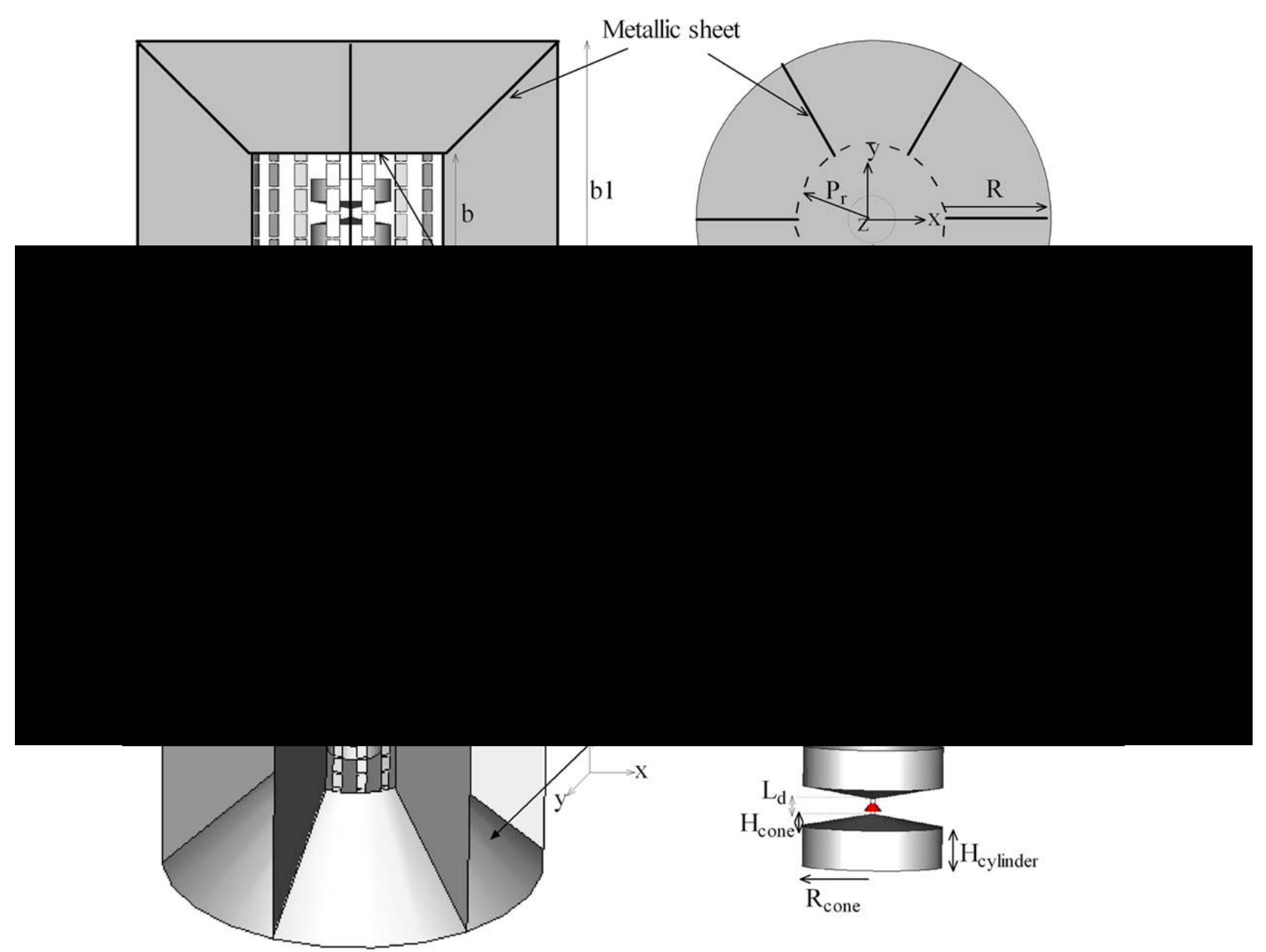

Fig. 3.15 Reconfigurable antenna design using FSS by Edalati and Denidni (2013). Clockwise from top Left, Side view, Top view, 3D view and the dipole at the center. 


\subsection{Pattern reconfigurable antennas using High Impedance Surfaces}

High impedance surfaces (HIS) are planar arrays of periodic metallic unit cells. The metal cells in the HIS can reflect EM waves without inflicting $180^{\circ}$ phase shifts at certain frequencies, as mentioned by Sievenpiper et al. (1999).

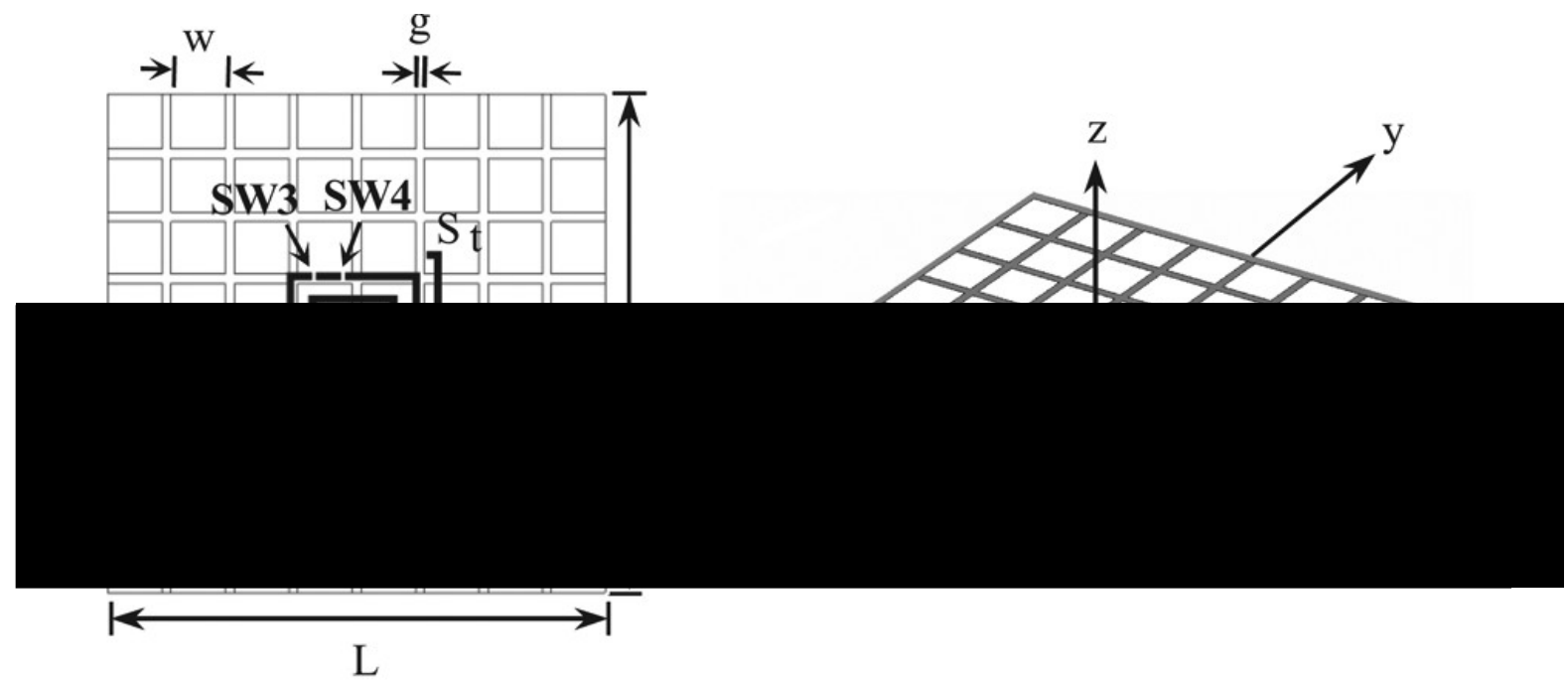

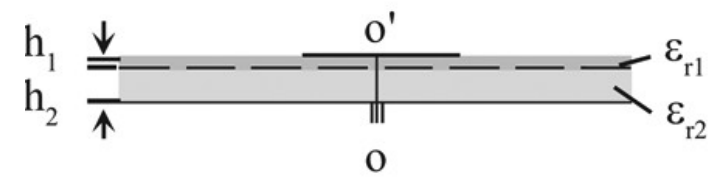

(a)

(b)

Fig. 3.16 Design of pattern reconfigurable planar spiral antenna over a HIS structure by Deo et al. (2009b).

HIS have been incorporated into the antenna substrates of some reconfigurable antennas reported such as the one reported in Deo et al. (2009b) and Huang et al. (2012). The pattern reconfigurable spiral antenna by Deo et al. (2009b) uses a HIS as an antenna ground plane to reduce the profile height of the antenna. This planar spiral antenna is similar to the spiral antenna presented in Jung et al. (2006) and Gong et al. (2013). It uses 4 switches $\left(S_{1}\right.$ to $\left.S_{4}\right)$ along the spiral arms of the antenna, which rests above a HIS structure embedded into its substrate, as shown in Fig. 3.16. The status of the 4 switches can be altered to create 16 pattern combinations with high Directivity above $7 \mathrm{~dB}$ in the frequency range between 3.3 $\mathrm{GHz}$ to $4.2 \mathrm{GHz}$.

Huang et al. (2012) proposes a reconfigurable HIS structure using graphene based blocks, for its dual loop reconfigurable antenna. Each unit cell in the HIS consists of a single layer 


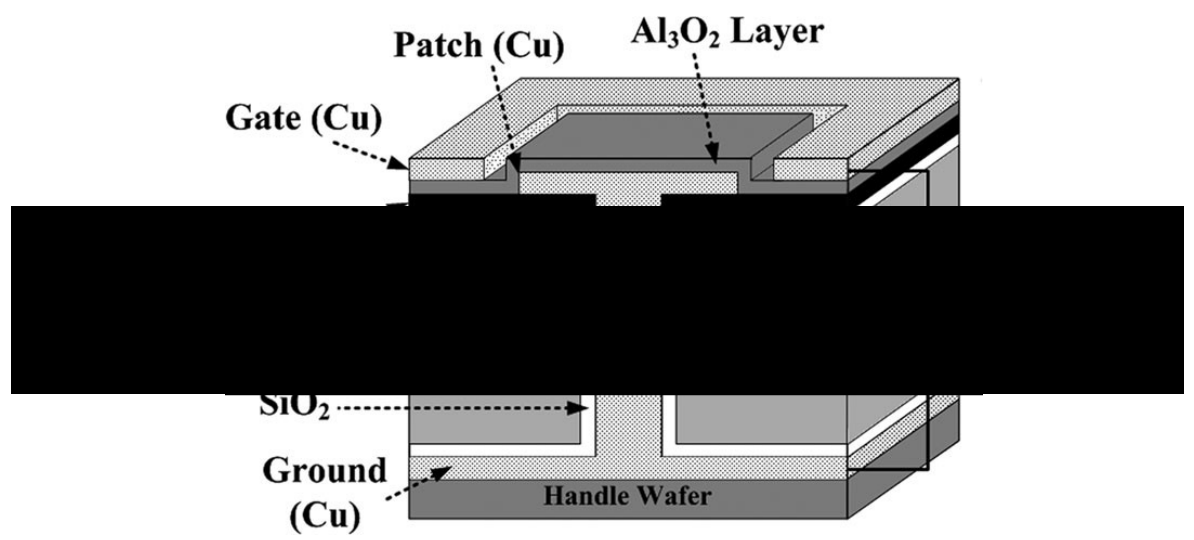

Fig. 3.17 Graphene based reconfigurable HIS block suggested by Huang et al. (2012).

graphene (SLG) square, as shown in Fig. 3.17. By doping and charging the gate voltage of the SLG unit, its surface conductivity can be made to be electrically tunable. For example, when the gate voltage $V_{g}$ is 0 , the graphene acts similar to a fully conductive material, effectively blocking the EM waves incident on it. Huang et al. (2012) proposes that each individual unit cell in this HIS can be made reconfigurable. Hence, the dual loop antenna above the HIS becomes pattern reconfigurable antenna. This antenna was designed for THz frequency range. It has approximately $34 \mathrm{dBi}$ Gain patterns for different cases of the switchable HIS states. It is not reported whether this method can be applied

\subsection{Pattern reconfigurable antennas using non-planar struc- tures}

In the work discussed before, the majority of the pattern reconfigurable antennas presented in literature were planar structures, except those discussed in Section 3.4, which used mostly cylindrical shaped FFS making the antenna bulky and impractical to be used in conformal or body-wearable applications. In this section, I study several pattern reconfigurable antennas that have non-planar structures, in order to study their usefulness in a body-wearable or a conformal application.

The antenna presented by Shi et al. (2012), uses an antenna structure that uses parasitic elements surrounding a monopole. This antenna structure is shown in Fig. 3.18a (Top). The electrical length of the monopole can be extended using a switch near the top half, as shown in Fig. 3.18b (Bottom Left). It is placed vertically at the center of a an octagonal wall surrounding it. Eight vertical parasitic elements are printed on these FR4 substrates, on each side of the surrounding wall as shown in Fig. 3.18b (Bottom Right). Eight RF switches 


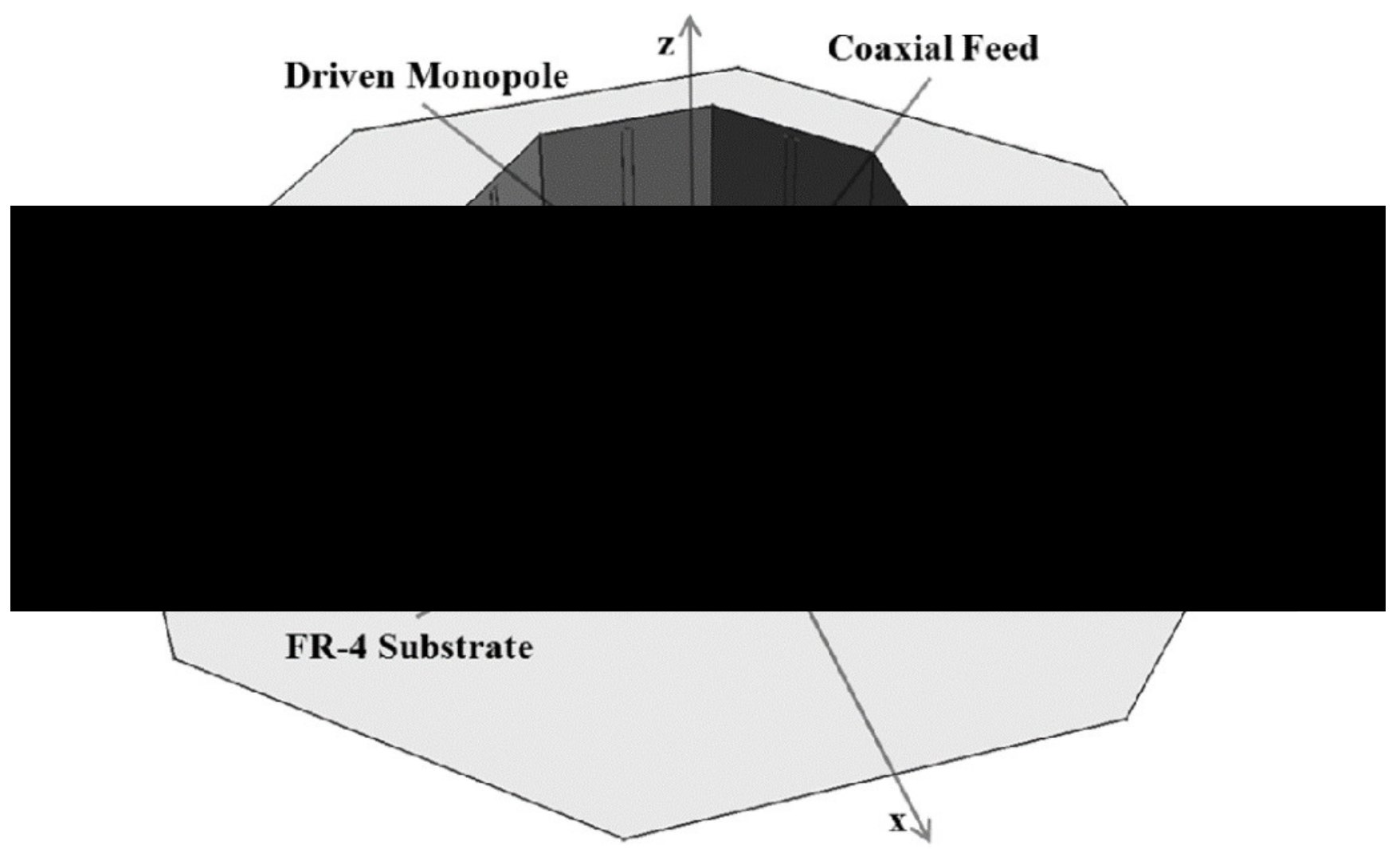

(a) Perspective design of pattern reconfigurable antenna structure.

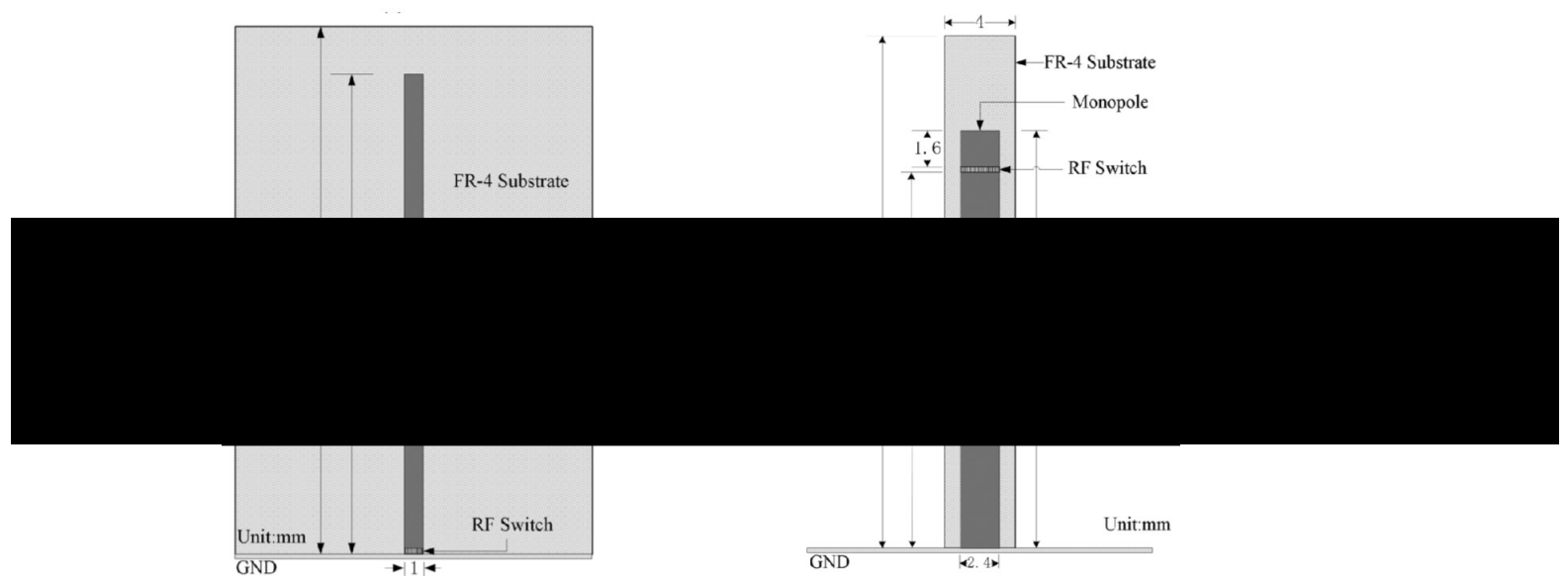

(b) Structure of the driven monopole at center (Left) and surround parasitic element (Right).

Fig. 3.18 Design of pattern reconfigurable antenna using switched printed elements by Shi et al. (2012). 
drive these parasitic elements. This wall has $40 \mathrm{~mm}$ radius and is $30 \mathrm{~mm}$ high. The parasitic elements can be switched ON or OFF, to create a reconfigurable pattern for the monopole antenna.

The antenna is operated in 8 modes corresponding to the ON/OFF status of each parasitic element. When one parasitic element on the wall is open-ended (i.e RF switch is OFF), the radiation beam of the monopole at the center can be directed towards this open ended parasitic element. Therefore, by switching OFF one parasitic element on the wall at a time, while keeping the rest OFF, the beam can be steered in $360^{\circ}$ azimuth direction one $45^{\circ}$ sector at a time. This antenna was designed for $2.4-2.8 \mathrm{GHz}$ frequency range. It has 9 switches including the switch at the monopole and creates 8 reconfigurable patterns. The main disadvantage of this antenna is its height of $30 \mathrm{~mm}$, which is in addition to the ground plane the structure sits on. Therefore it has around $0.25 \lambda$ profile height and also requires 9 switching control voltages.

Lee et al. (2013) presents a 3D reconfigurable antenna for $2.4-2.5 \mathrm{GHz}$. It has two planar printed wings in the $\mathrm{X}-\mathrm{Z}$ plane and the $\mathrm{Y}-\mathrm{Z}$ plane. These two wings have two folded dipoles printed on them. The first wing also has a triangular loop in addition to the printed dipole. It can steer the beam in the $\mathrm{X}-\mathrm{Y}$ and $\mathrm{X}-\mathrm{Z}$ planes using $4 \mathrm{RF}$ switch configurations. The measured Gain patterns of the antenna has a Gain between $1.5 \mathrm{dBi}$ to $2.06 \mathrm{dBi}$ and can steer the beam in the azimuth direction in the range of $0^{\circ}<\phi<90^{\circ}$. Due to its orthogonal nature of its two wings, it is not suitable for a conformal application. Li et al. (2011a) presents a folded loop antenna designed for mobile handsets. This antenna consists of a loop (Fig. 3.19 (Top)), which is then folded as shown in Fig. 3.19 (Bottom). By using two p-i-n diode switches $S_{1}$ and $S_{2}$, the loop can be shorted to ground at two points, thereby altering the length of the loop. This changes the resonating frequency for the folded antenna. It has 2 configurations for the two switches: ON-OFF and OFF-ON. In the ON-OFF state, it resonates in the GSM850, GPS and WiFi frequencies, while in the OFF-ON state, it resonates in the GSM900, DCS, PCS, and UMTS freuency bands. The reported radiation patterns of the anntenna by Li et al. (2011a) show near omni-directional patterns with low directivity. The antenna proposed by $\mathrm{Li}$ et al. (2009) also has a similar structure and works on the same principles.

\subsection{Pattern reconfigurable square loop antenna}

Majority of the previous work studied in this chapter make use of p-i-n diodes, RF switches. FET switches or varactors in order to achieve pattern reconfigurability. However, when a RF switch or a p-i-n diode is excited, it results in a change in the current distribution pattern 


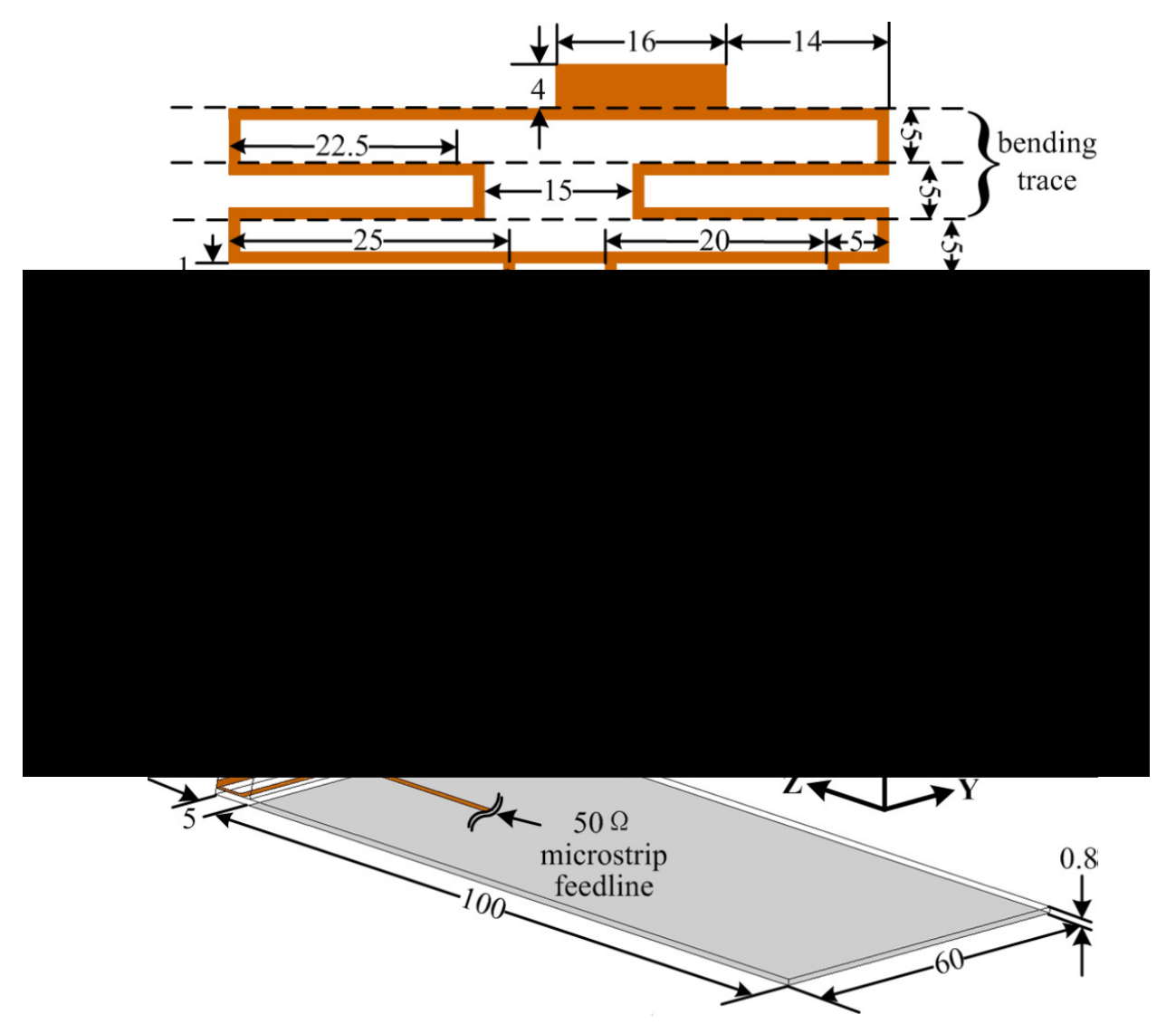

Fig. 3.19 Design of heptaband reconfigurable loop antenna by Li et al. (2011a). (Top) the loop antenna dimensions before folding. (Bottom) the folded loop antenna integrated with feedline. 
along the radiating element. Quite often, this causes a change in the polarisation of the antenna. This effect is called polarisation randomness (Mehta and Mirshekar-Syahkal, 2007). Therefore, most of the reconfigurable antennas studied previously which used switching elements such as p-i-n diodes, RF switches, FET switches or varactors, have a polarisation dominance in one direction (i.e $E_{\theta}$ or $E_{\phi}$ ).

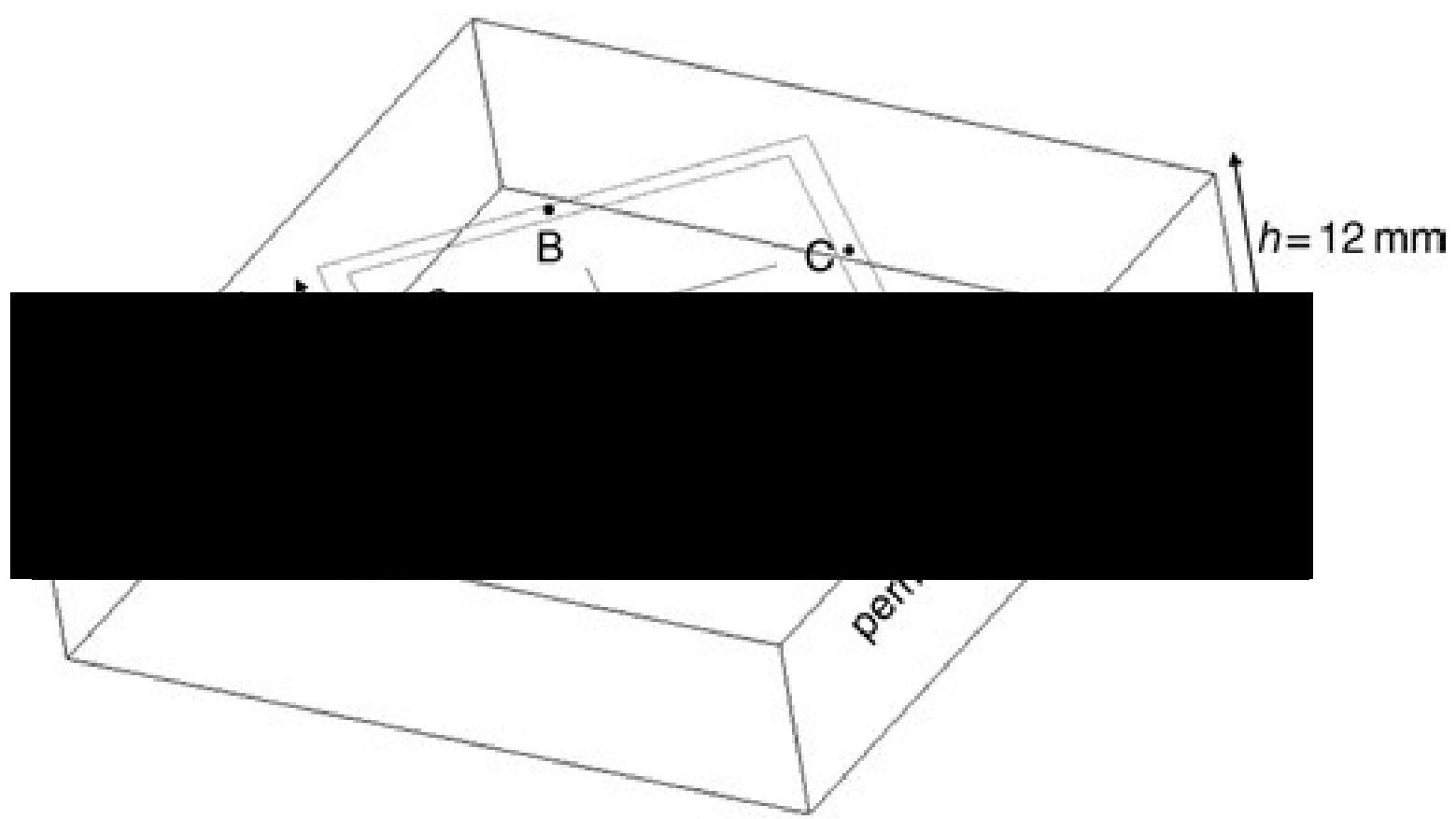

Fig. 3.20 The design of SLA by Mehta and Mirshekar-Syahkal (2007).

The square loop antenna (SLA) with 4 feed termination proposed by Mehta and MirshekarSyahkal (2007) creates a reconfigurable beam pattern which can be steered in the four quadrants of space in the azimuth direction. It overcomes the problem of polarization randomness by having an electronically controlled single pole four throw (SP4T) RF switch which directs the RF input $\left(R F_{\text {in }}\right)$ to the relevant feed of the SLA. Since the SP4T switch is used before the feed terminations, the RF feed to the antenna does not undergo polarisation randomness while travelling along the antenna's radiating arms, as in the case of p-i-n diodes and switches placed along the antenna's radiating elements in previous work. The design of the SLA is shown in Fig. 3.20. It has a $12 \mathrm{~mm}$ thick substrate on which a $1.5 \mathrm{~mm}$ thick and $30 \mathrm{~mm}$ wide square loop has been etched. The SLA is fed at 4 points at the center of each arm of the square loop. This design is optimized for $5.2 \mathrm{GHz}$ operation. When feeding was done at one of the 4 points, it produces a narrow beam with high Gain $(9 \mathrm{dBi})$ which has a tilt angle of $\theta=48^{\circ}$. Due to the symmetry of this antenna's design, each port is identical 


\section{Literature review}

to the other three ports. Therefore, when feeding is done at each port separately, it would produce identical beams in the $\phi=45^{\circ}, \phi=135^{\circ}, \phi=225^{\circ}$ and $\phi=315^{\circ}$.

However, the simulated work by Mehta and Mirshekar-Syahkal (2007) involves using an infinite ground plane and an infinite substrate, which is not the case in the original design. Furthermore, the 4 feed points are fed from the top of the SLA arms which cannot be implemented in practice.

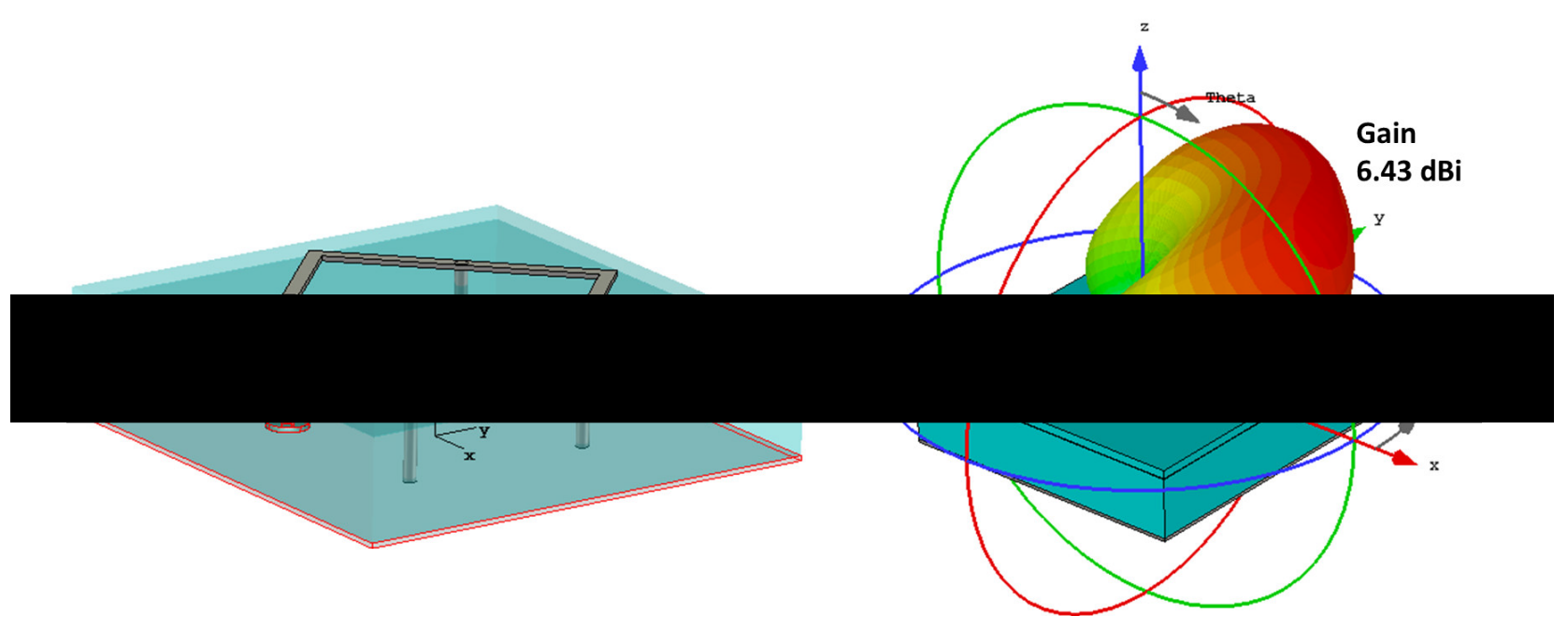

Fig. 3.21 The SLA with short circuited feed terminations design (Left) and the simulated farfield Gain pattern (Right) by Pal et al. (2008).

The antenna reported by Pal et al. (2008) introduces one SMA feed and 3 short circuited feeds (vias connected to ground), for the 4 port SLA mentioned in Mehta and MirshekarSyahkal (2007). The antenna design and its simulated farfield pattern are shown in Fig. 3.21. This antenna produces a $6.41 \mathrm{dBi}$ Gain pattern (Fig. 3.21 (Right)) at $4.5 \mathrm{GHz}$. Its maximum Gain pattern direction has a tilt angle of $\theta=45^{\circ}$. It produces a directive pattern across and away from the fired port shown in Fig. 3.21 (Left). The beamwidth of the antenna's gain pattern is wide enough to cover one quadrant in space $\left(0^{\circ}<\phi<90^{\circ}\right)$ at the tilted angle of $\theta=45^{\circ}$. This antenna is a good candidate for multipath mitigation applications as it covers a $90^{\circ}$ area in the azimuth plane and has less than $-9 \mathrm{dBi}$ gain in any other quadrant. Therefore, it will be able to reject multipath or jamming signals coming from other directions. However, the design proposed by Pal et al. (2008) has only 1 feed which is connected to $R F_{i n}$. Hence, this structure does not have beam reconfigurability due to the other 3 feeds being short circuited.

Later reported work on this pattern reconfigurable SLA has involved the 4 feed RF feeding mechanism (Deo et al., 2011a; Pal et al., 2013a) as shown in Fig 3.22a (Top). Pal et al. (2013a) has reported a feeding network that uses a 4-way Wilkinson power divider/combiner 


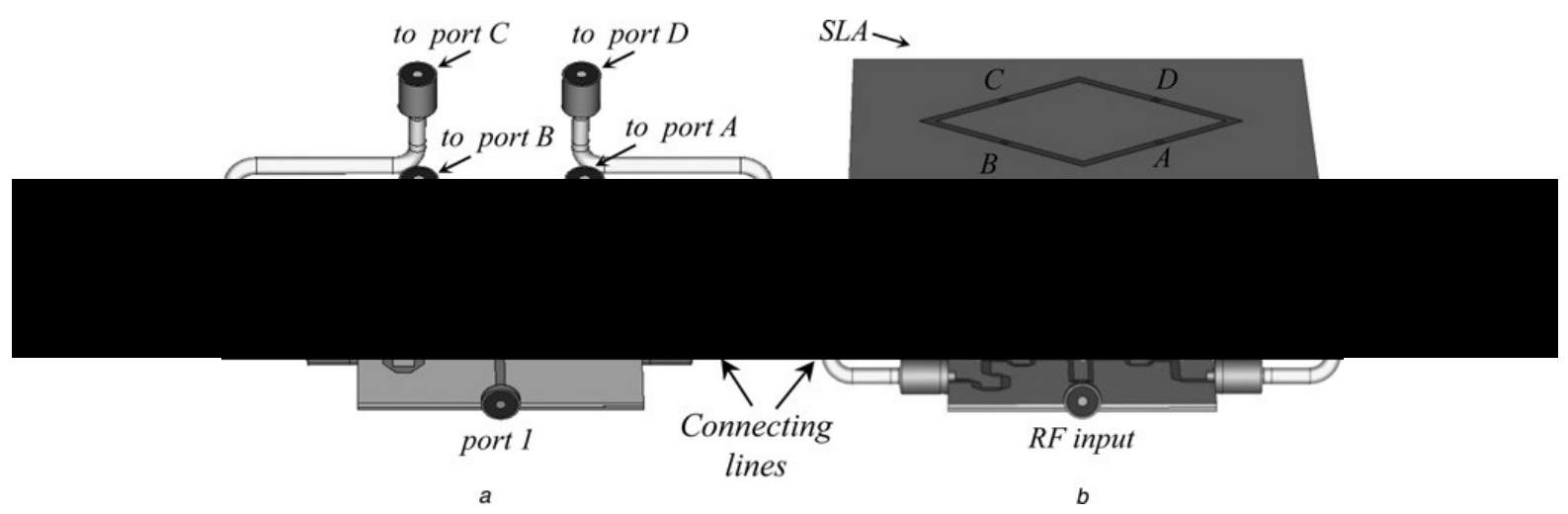

(a) The feeding mechanism for the 4 port SLA by Pal et al. (2013a).
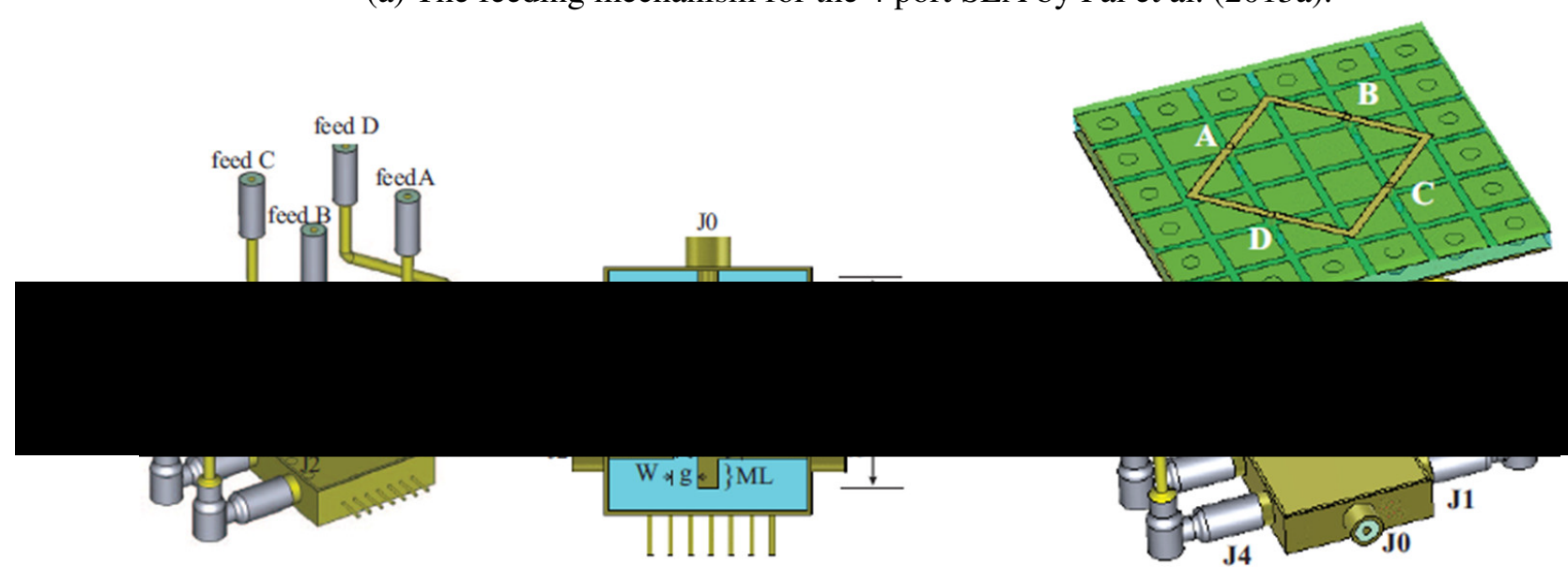

(b) The feeding mechanism using RF switch for the 4 port SLA by Deo et al. (2011a).

Fig. 3.22 Feeding mechanisms for the 4 port SLA proposed by Pal et al. (2013a) and Deo et al. (2011a) 
and delay lines (Fig. 3.22a (Top)). The $R F_{\text {in }}$ of the port 1 in this feeding network is divided into 4 equal parts and routed to ports 2 through 5 , which in turn feed the SLA's 4 ports: A, B, C and D. Pal et al. (2013a) states that by designing this power divider with delay lines of different lengths, sequential $90^{\circ}$ phase shifts can be introduced to the 4 ports (i.e $\phi=0^{\circ}, \phi=90^{\circ}, \phi=180^{\circ}$ and $\phi=270^{\circ}$ ). The phase shift introduced by a transmission delay line of length $l$ can be expressed as:

$$
\theta=\frac{l}{\lambda_{g}} \times 360^{\circ}
$$

Therefore, by using this feeding mechanism, since the Wilkinson power divider divides the power equally and feeds the 4 ports (A,B,C and D) of the SLA simultaneously with sequential $90^{\circ}$ phase shifts introduced by the delay lines, a circularly polarised beam pattern can be generated. The generated beam pattern is axial (i.e along the Z-axis. $\theta=0^{\circ}$ ).

The feeding mechanism proposed by Deo et al. (2011a) consists of an electronically controlled SP4T RF switch (Fig. 3.22b). For the purpose of simulation, the SP4T RF switch is considered as a p-i-n diode controlled switch which completes the path from $R F_{\text {in }}$ to the respective port, when the diodes are switched ON. Unlike the feeding mechanism in Pal et al. (2013a), this switch cannot generate a CP axial beam. It however, generates an identical beam pattern for each port, when fired. It also has the ability to generate a doughnut shaped (monopole like pattern with only the top half), when all 4 ports are simultaneously fired with $0^{\circ}$ phase shifts.

Deo et al. (2010) has presented a pattern reconfigurable 4-port SLA on a hybrid high impedance surface (HHIS). This design is shown in Fig. 3.23. This HIS design differs from the previous reported work on reconfigurable antennas using HIS in section 3.5 (Deo et al., 2009b; Huang et al., 2012) as it only uses vias to short the outer ring of the square metal patches to the ground plane. The rest of the square metal patches inside the structure are not shorted. There are four conducting (pins) feeds feeding the four arms of the SLA and they are positioned through the gaps between the square metal patches in the HHIS. By using this HHIS, the antenna's profile height has been reduced from $12 \mathrm{~mm}\left(\lambda_{0} / 3.21\right)$ previously to $4.69 \mathrm{~mm}\left(\lambda_{0} / 13.61\right)$. The SLA also has perimeter length of $2.67 \lambda_{g}$ at $4.7 \mathrm{GHz}$. However, this thickness reduction had been achieved at the expense of a more complex design due to the numerous vias which make the antenna more heavy and difficult to manufacture. Therefore, other methods of profile height reduction of the SLA has been explored in previous work (Pal et al., 2012a).

Sievenpiper et al. (1999) states that if an antenna was placed too close to the ground plane, the antenna's radiated fields will be cancelled out by the reverse image currents in the 


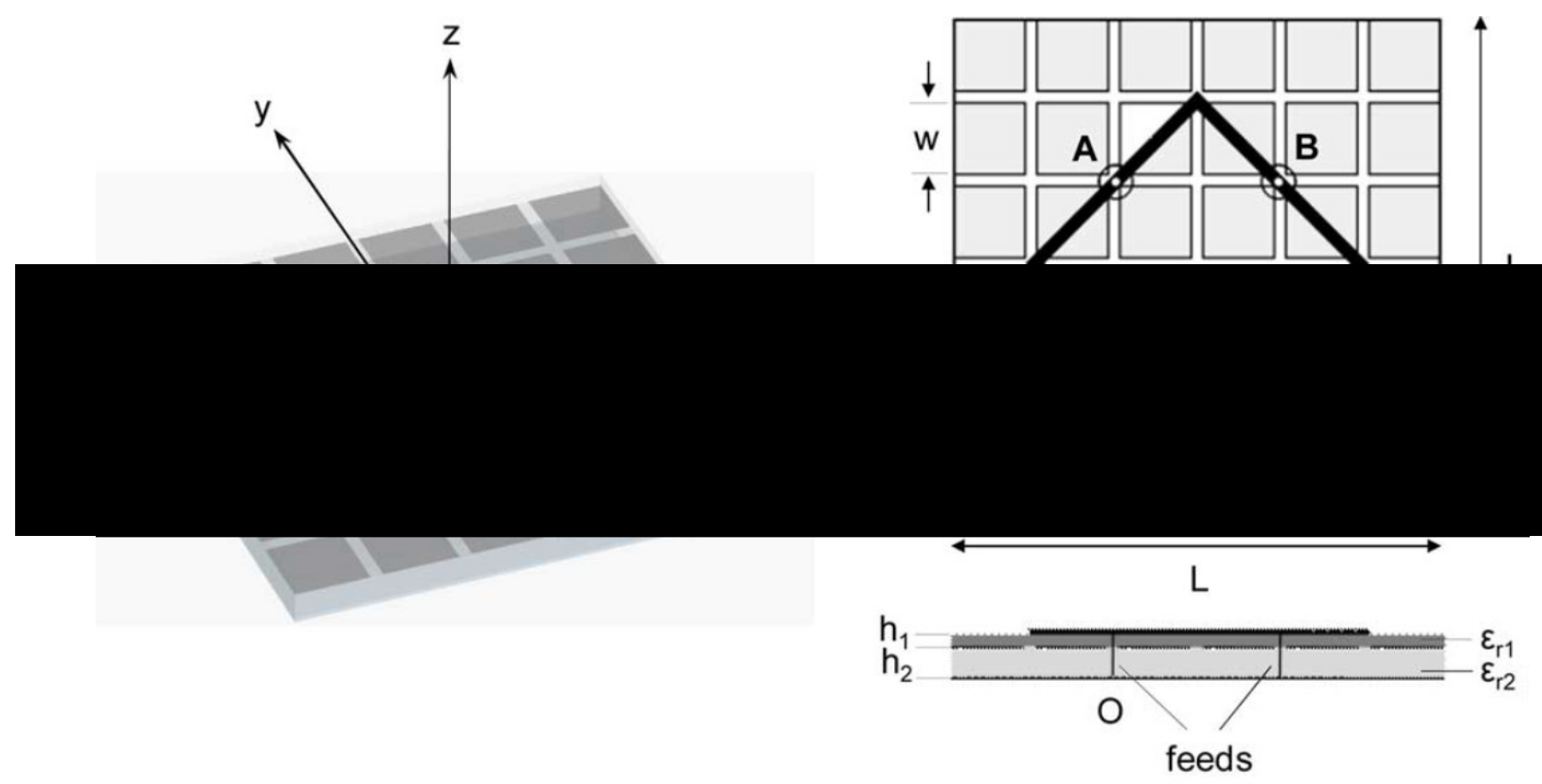

Fig. 3.23 The SLA on a hybrid high impedance surface (HHIS) by Deo et al. (2010).

conducting ground plane. This would result in poor antenna efficiency. (Pal et al., 2012a) has managed to overcome this problem by reducing the antenna's substrate thickness and feed the SLA's radiating arms using capacitively coupled feeding. The 4 feeding pins are connected to rectangular patches in close proximity to the center of each of the SLA's arms. These capacitively coupled patches will introduce a capacitance to the antenna's input impedance to cancel out the inductance created by the feeding pins and the close proximity of the ground plane. This antenna has $3.02 \mathrm{~mm}\left(\lambda_{0} / 19\right)$ profile height at $5.2 \mathrm{GHz}$. It also has a radiation pattern with high directivity $(9.1 \mathrm{~dB})$. However, when capacitively coupled feeding was introduced, the impedance bandwidth has been limited to $350 \mathrm{MHz}$.

Later work on the pattern reconfigurable SLA has involved the design of a dual band capacitively coupled SLA (DBCCSLA) by Pal et al. (2014a,b, 2013b). This design, which is shown in Fig. 3.24 introduces a second co-centered square loop which will radiate at a lower frequency than the square loop inside it. This creates a dual frequency band for the 4-port SLA which is fed via capacitively coupled rectangular patches positioned in between each arm of the two loops. The frequency response of the return loss coefficient $\left(S_{11}\right)$ of DBCCSLA is shown in Fig. 3.25. It has two resonances at $4.1 \mathrm{GHz}$ and $5.2 \mathrm{GHz}$ corresponding to the resonant from the outer loop and the inner loop. It has two sharp directive beams of $9.2 \mathrm{dBi}$ Gain. However its impedance bandwidth at the two frequency bands are $130 \mathrm{MHz}$ and $160 \mathrm{MHz}$ respectively. This is a result of the capacitively coupled feeding explained earlier. 


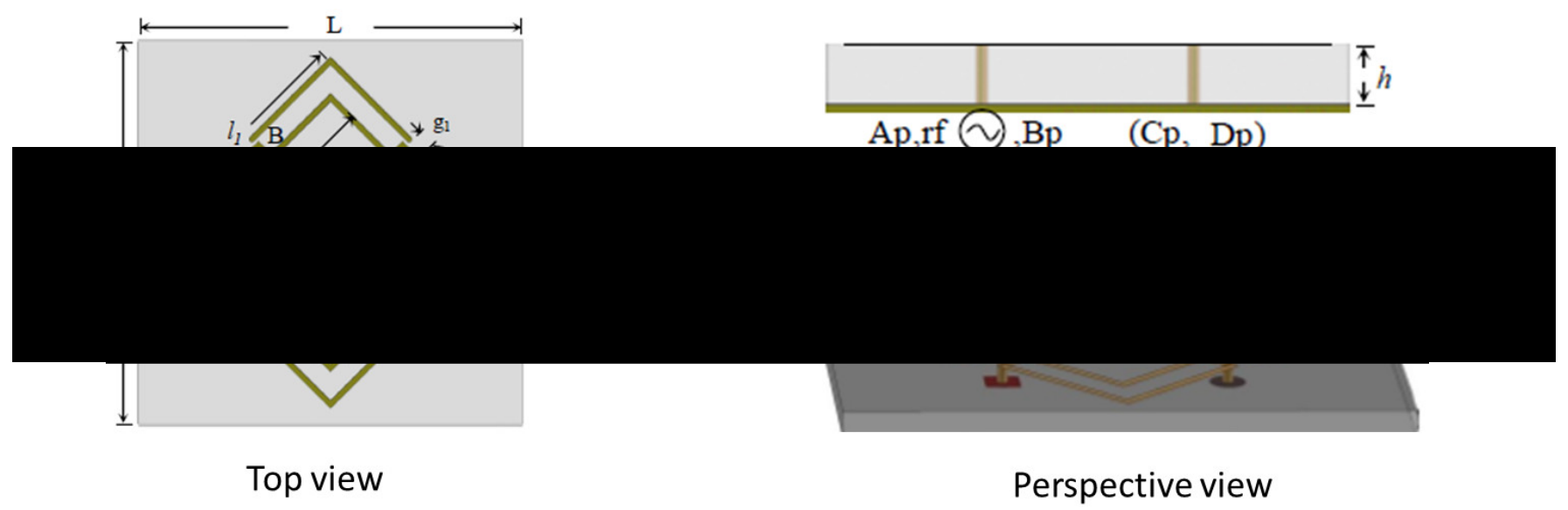

Fig. 3.24 The dual band capacitively coupled SLA design by Pal et al. (2013b).

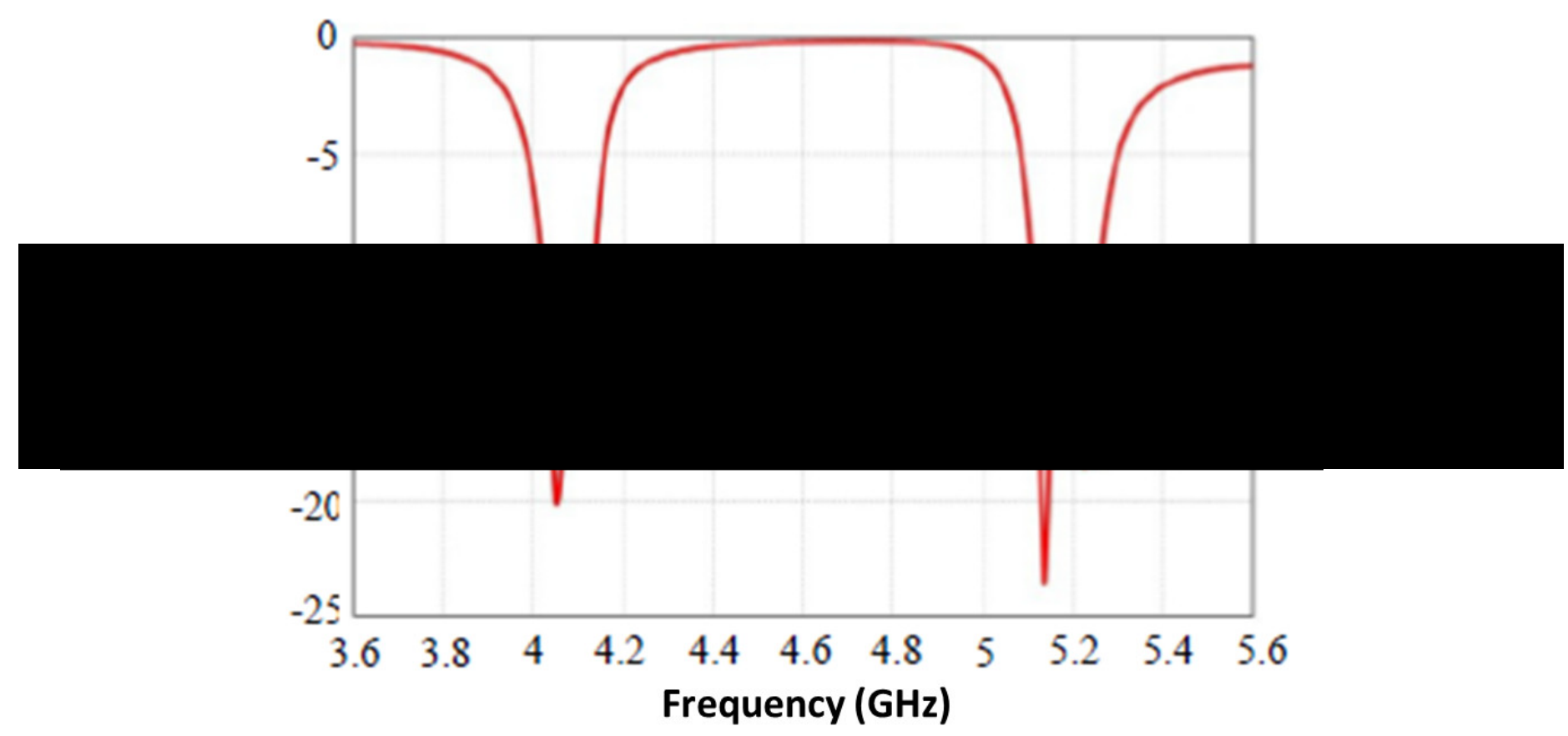

Fig. 3.25 The frequency response of the return loss coefficient of the DBCCSLA by Pal et al. (2013b). 
The low impedance bandwidth problem in the DBCCSLA has been overcome in later reported work by Pal et al. (2014b) and Pal et al. (2014a). This has been achieved by creating a gap at the center of each radiating arm of the SLA, which is fed via capacitively coupled feeding. The air gap created in the centre of the SLA's arm gives rise to an additional 'loaded resonance' along with an 'unloaded resonance' whose frequencies are close to each other (Pal et al., 2014a). This creates an overlapping frequency band at the resonances that helps to create a wider impedance bandwidth. Remarkably, these two loaded modes introduced by the air gap in the middle have similar radiation patterns as well. Therefore, the radiation pattern throughout the two frequency bands maintains a similar shape. The antenna presented in Pal et al. (2014b) has two resonant frequency bands at 3.8 GHz and 4.7 GHz. The DBCCSLA profile height is $3.04 \mathrm{~mm}$ and at the lower frequency band of $3.8 \mathrm{GHz}$, this corresponds to a height of $\left(\lambda_{0} / 26\right)$. The antenna's radiation patterns have $9.3 \mathrm{dBi}$ and $9.2 \mathrm{dBi}$ Gains respectively for the two resonant frequency bands.

\subsection{Conformal pattern reconfigurable antennas}

Pattern reconfigurable antennas have several applications including multipath mitigation, direction of arrival estimation, beam scanning, jamming avoidance etc. However, due to the variety of applications and scenarios that these antenna can be implemented in, recent focus has shifted to creating conformal pattern reconfigurable antennas for on-body or vehicular applications. According to the IEEE definitions on antennas, a conformal antenna is: "an antenna that conforms to a surface the shape of which is determined by considerations other than electromagnetic: for example, aerodynamic or hydrodynamic" (IEEE, 2014).

In the previous section several techniques used for creating pattern reconfigurable antennas were discussed. Among the reviewed work, use of p-i-n diodes, RF switches and varactors and FSS were prominent. Antennas that use $\mathrm{p}-\mathrm{i}-\mathrm{n}$ diodes, RF switches or varactors on the surface of the antenna's radiating elements are planar in nature. It is difficult to make such antennas conformal due to the rigid physical nature of these elements and the necessity to have wires in close proximity to drive them.

Jais et al. (2013) has presented a pattern reconfigurable textile antenna for $2.45 \mathrm{GHz}$ wireless body area networks (WBAN). This antenna uses a central circular patch which is fed using a SMA's coaxial probe at the center. It has 4 circular patches around it at $\phi=0^{\circ}$, $\phi=90^{\circ}, \phi=180^{\circ}$ and $\phi=270^{\circ}$. The central patch is connected each of the four patches using a planar conducting arm. There are 4 RF switches along each of the 4 arms to switch each outer circular patch ON/OFF to create 4 reconfigurable patterns which can be steered to each of the 4 quadrants. Its reported radiation patterns contain $6.8 \mathrm{~dB}$ directivity. This 


\section{Literature review}

antenna is thin (1.2 mm height) and has a surface area of $88 \mathrm{~mm}^{2}$. However, It has 8 wires running across its surfaces to control the $4 \mathrm{RF}$ switches.

Javed et al. (2015) has presented a reconfigurable body wearable bluetooth and WiMAX antenna which uses commonly found jeans fabric material as its substrate. This antenna uses $\mathrm{p}$-i-n diode switching to connect the two rectangular patches to the central circular patch, which is fed using a planar feed. This is shown in Fig. 3.26. It has a circular patch connected to two rectangular patches. The antenna operates in two modes: when one $\mathrm{p}-\mathrm{i}-\mathrm{n}$ diode is $\mathrm{ON}$, the other is in OFF state and vice versa. This creates a two beam reconfigurable pattern operated by two p-i-n diodes. This antenna has a directive pattern but has limited beam steerability.

The antenna presented by (Kang and Jung, 2015) also uses fabric material $\left(\varepsilon_{r}=1.35\right.$, $\delta=0.02$ and thickness $1.5 \mathrm{~mm}$ ) for its reconfigurable antenna with a WiFi module. It uses $\mathrm{p}$-i-n diodes for pattern reconfigurability for the proposed U-shaped slot antenna with proximity feeding. It has a ground plane backing on the reverse side of the fabric where the antenna is positioned. Due to the ground plane it has low specific absorption rate (0.68 - 0.98 $\mathrm{W} / \mathrm{kg}$ ) and has a good directinve beam pattern. This antenna too uses $\mathrm{p}-\mathrm{i}-\mathrm{n}$ diodes on the surface of the radiating elements. Later reported work by Ha and Jung (2011) presents the reconfigurable 3D patterns of this antenna in 3 modes: axial pattern (along $+Z$-axis, in the direction of $\phi=0^{\circ}$ and in $\phi=180^{\circ}$ direction). The 3 simulated patterns have directivity between $6.15 \mathrm{~dB}$ to $5.65 \mathrm{~dB}$ at $6 \mathrm{GHz}$.

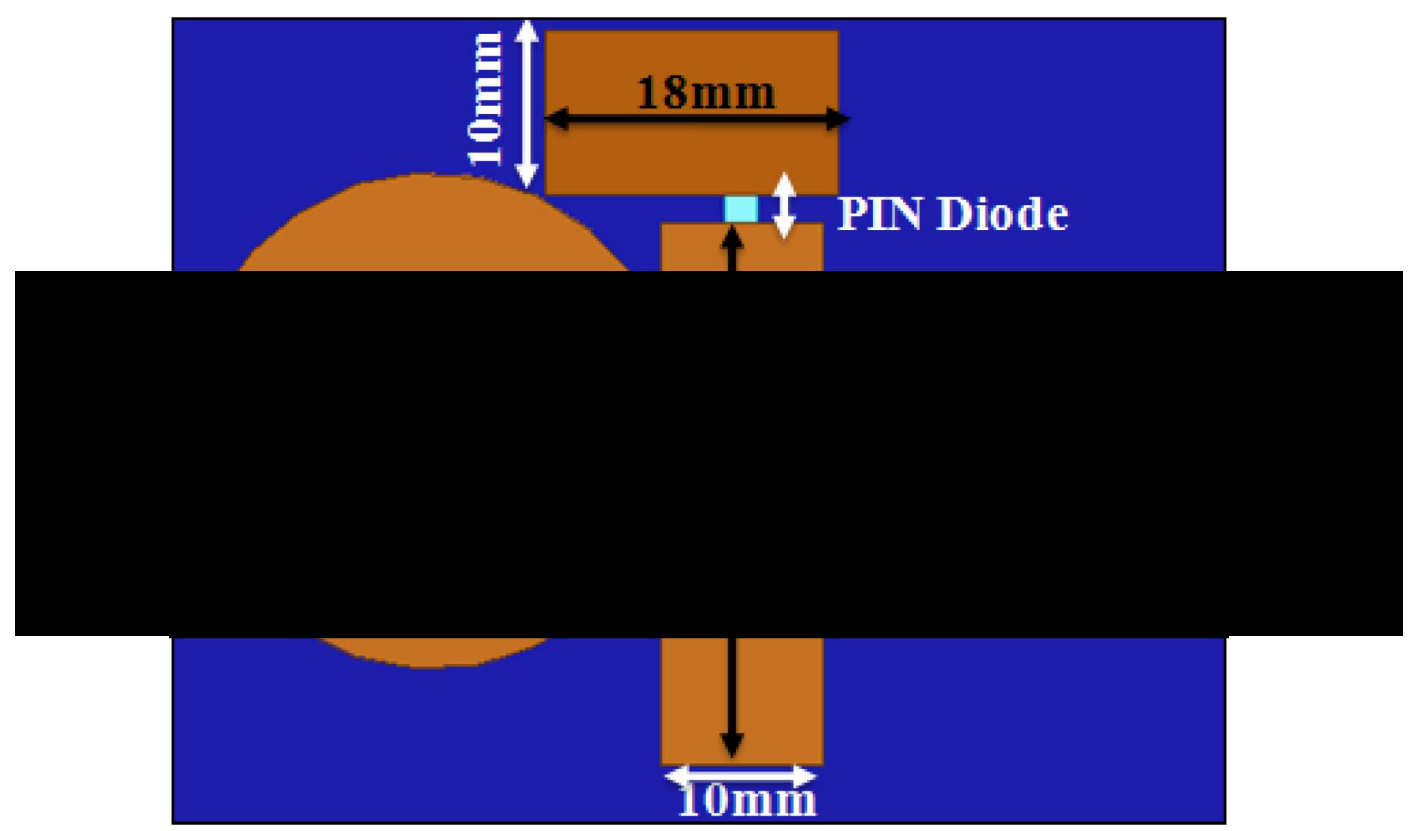

Fig. 3.26 Body wearable reconfigurable antenna by Javed et al. (2015). 


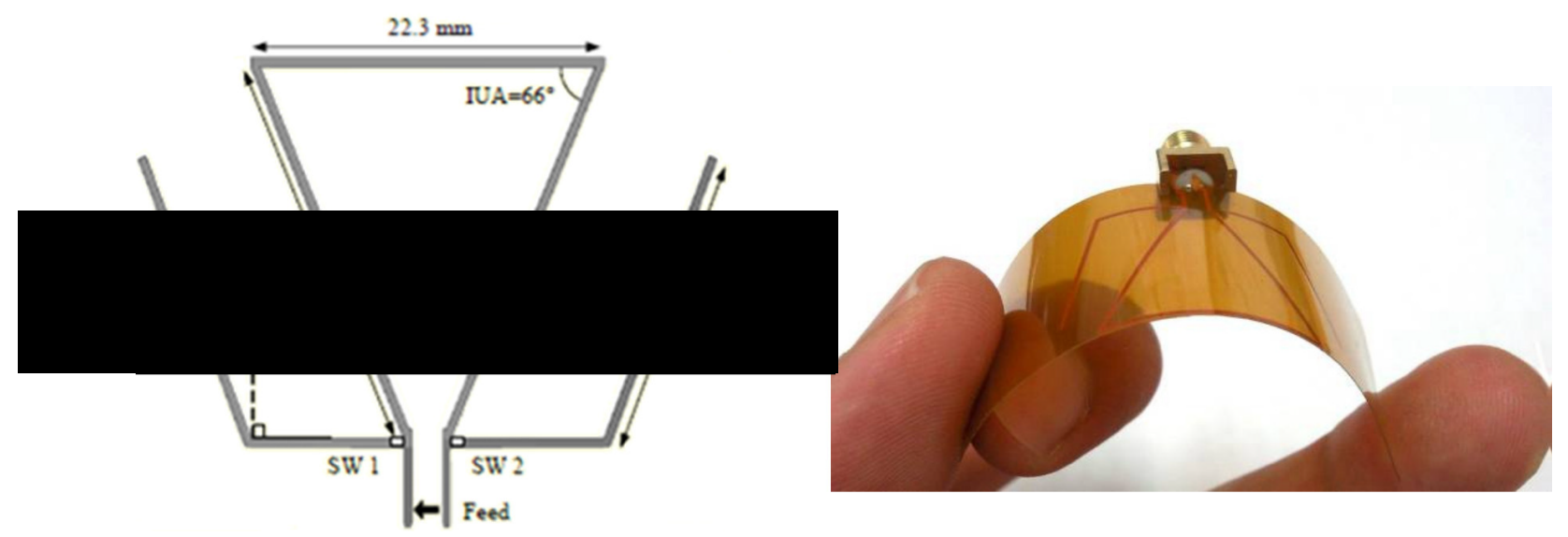

Fig. 3.27 Flexible body wearable reconfigurable antenna design (Left) by Ha and Jung (2011) and the prototype (Right).

A flexible conformal reconfigurable antenna for body worn applications was proposed by Ha and Jung (2011). This antenna consists of an inverted trangular loop with two wings on either side similar to a folded dipole structure, as shown in Fig. 3.27 (Left). The designed antenna has two switches connecting the triangular loop to either wing of the folded dipole. This design is similar to the antenna by Lee et al. (2013) discussed earlier except for the second folded dipole which is orthogonal to the triangular loop and the first folded dipole plane, which is not present in this antenna. This antenna is designed for $2.5 \mathrm{GHz}$ and has a operating bandwidth of $40 \mathrm{MHz}-60 \mathrm{MHz}$. When both the switches are in OFF-OFF configuration, it creates a dipole-like Gain pattern with a maximum directivity of $2.48 \mathrm{~dB}$. In the other two configurations (OFF-ON and ON-OFF), the antenna produces a $2.11 \mathrm{~dB}$ and $1.96 \mathrm{~dB}$ directive patterns with their maximum Gain in the $\phi=130^{\circ}$ and $\phi=230^{\circ}$ directions, respectively.

Ha and Jung (2011) has also studied the effects on this antenna's radiation pattern when the substrate undergoes bending in one plane (i.e singular curved surface). Increase in bending around the Y-axis results in a change in the maximum Gain and its direction of the radiation pattern. For 2 levels of increasing bending studied, the direction of maximum Gain changes from $\phi=-50^{0}$ (planar) to $\phi=-18^{0}$ and $\phi=-28^{0}$ (maximum bend). Its directivity changes from $0.8 \mathrm{~dB}$ to $1.6 \mathrm{~dB}$ during this operation. The prototype presented in this work however, does not contain the implementation of the two switches that are needed for pattern reconfigurability. It is not reported how the antenna would perform with the increases in bending when the switches are implemented on the flexible PCB material which is used as the substrate in this work. 
A flexible conformal square loop antenna with a single feed is presented by Damis et al. (2016). This antenna uses two smaller circular patches along the side of the square loop where the antenna is fed at. There are two further circular patches on the opposite arm of the square loop. The reported work has very high maximum Gain of $12 \mathrm{~dB}$ in the $\phi=310^{\circ}$ azimuth direction and an unspecified beam tilt angle $\left(0^{0}<\theta<90^{\circ}\right)$. It has a very good input impedance matching at $2.45 \mathrm{GHz}$. Although this antenna has one single pattern and is not reconfigurable, the reported results are very encouraging for conformal applications. Further study is needed whether the type of 4-feed SLA reported in Deo et al. (2011b) can be implemented in this conformal manner to achieve such high Gain.

The use of HIS is not a technique that provides pattern reconfigurability to an antenna. Instead, it is used mainly for antenna profile height reduction, which could be an advantage in conformal applications. However, due to their complex structure and the difficulty in manufacturing substrates embedded with HIS with vias, little or no attempt had been made to design conformal antennas using HIS.

Palikaras et al. (2011) and Feresidis et al. (2011) have reported conformal antennas using HIS structures. These base station antenna are conformal to cylindrical surfaces. They do not use vias to connect the metal patches to the ground plane. Li et al. (2013) has presented a pattern reconfigurable antenna for body wearable applications. This antenna uses a HIS structure. In the simulated model reported in this work, the pattern reconfigurable antenna rests above the HIS plane on a layer of air. It uses p-i-n diodes for pattern reconfigurability. Although the simulated results validate this antenna's usability in a body wearable application, the reported prototype is a rigid planar structure. Hence, it is not known whether this antenna can be made conformal since it includes a HIS structure.

The reconfigurable antennas using FSS previously discussed, all have 3D structures. Therefore they are not useful in the implementation of conformal antennas.

\subsection{Plasma reconfigurable antennas}

All the antennas previously discussed in this chapter contained fixed metal radiating elements which produce a reconfigurable beam due to switching between its elements. One of the latest developments in the field of reconfigurable antennas is the design of plasma antennas. Compared to conventional antennas which have fixed metal radiating elements, plasma antennas contain gaseous elements that exploit ionized gas to transmit and receive EM waves.

As mentioned by Melazzi et al. (2017), in a plasma antenna, the plasma discharge parameters such as its density can be tuned. Since the plasma antenna's properties can be changed dynamically, this provides a promising alternative to conventional antennas where 
reconfigurability is required. Dagang et al. (2016a) state that another advantage of using plasma antennas is that the column of plasma can be switched ON and OFF rapidly in microseconds. As mentioned by Alexeff et al. (2006), the ionized gasses used in plasma antennas provide several other advantageous such as camouflage from waves, coupling reduction, interference reduction, noise reduction and radar cross section reduction.
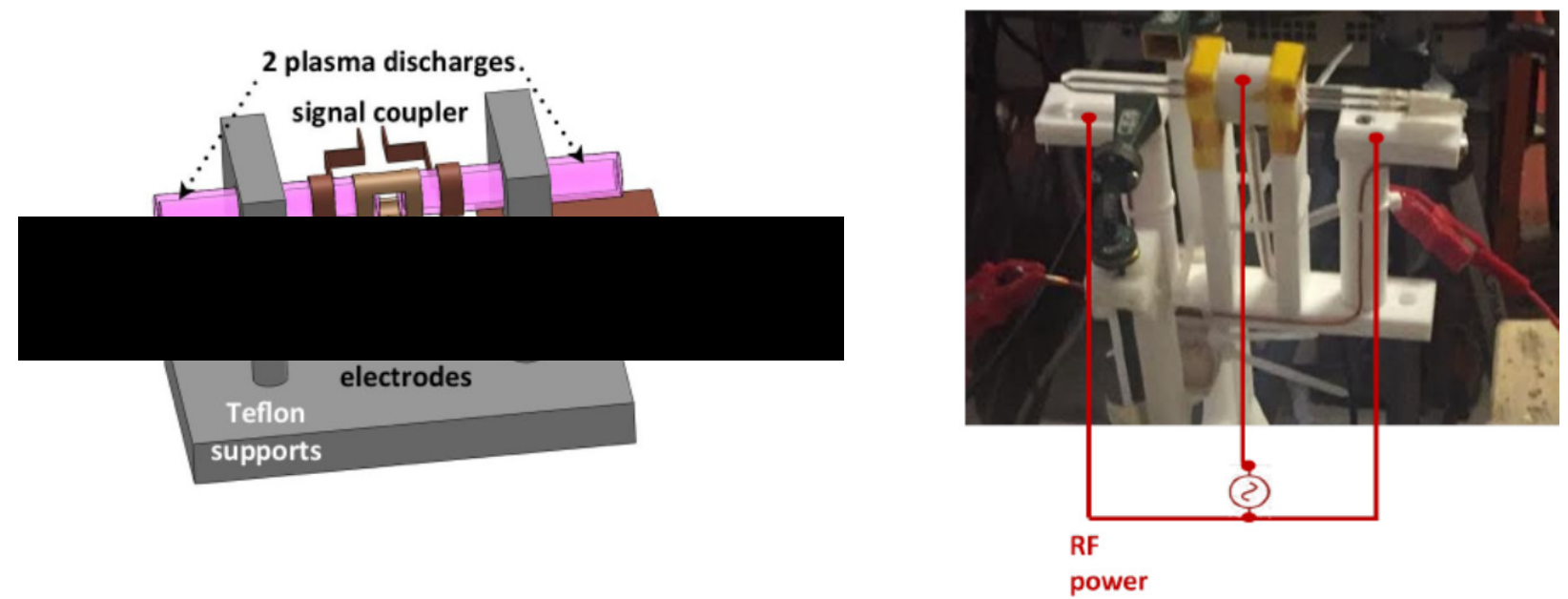

Fig. 3.28 Reconfigurable dipole plasma antenna design (Left) by Melazzi et al. (2017) and the prototype (Right).

Due to the rigid structure of the plasma tube most published work on reconfigurable plasma antennas involved the use of a monopole-like plasma tubes (Barro et al., 2017; Dagang et al., 2016b; Halili et al., 2013; Jaafar et al., 2017; Zali et al., 2013). However, the plasma dipole antenna presented in Melazzi et al. (2017) contains a horizontal plasma tube which is excited by a RF signal coupler in the middle as shown in Fig. 3.28. The plasma is discharged using a two high voltage electrodes, at the two ends of the plasma tube containing Argon gas. In the experimental work reported by Melazzi et al. (2017) it was stated that the plasma density is non-uniform in the actual gas discharge and hence, influenced the antenna's radiating performance.

Ghaderi et al. (2017) has presented a folded dipole antenna. In this antenna, the folded tube containing the plasma is excited by a metallic coupler at the center using a $75 \Omega$ transmission line. This antenna operates at a center frequency of $270 \mathrm{MHz}$. Mansutti et al. (2016) has presented a hybrid Metal and Plasma Yagi-Uda antenna which contains two radiating metal elements and two radiating plasma tubes which provide reconfigurability to the antenna. Compared with the previously mentioned monopole and dipole plasma antennas which were designed for MHz frequencies, this Yagi-Uda antenna operates at $1.5 \mathrm{GHz}$ and displays very close performance to classical metal Yagi-Uda antennas. 


\section{Literature review}

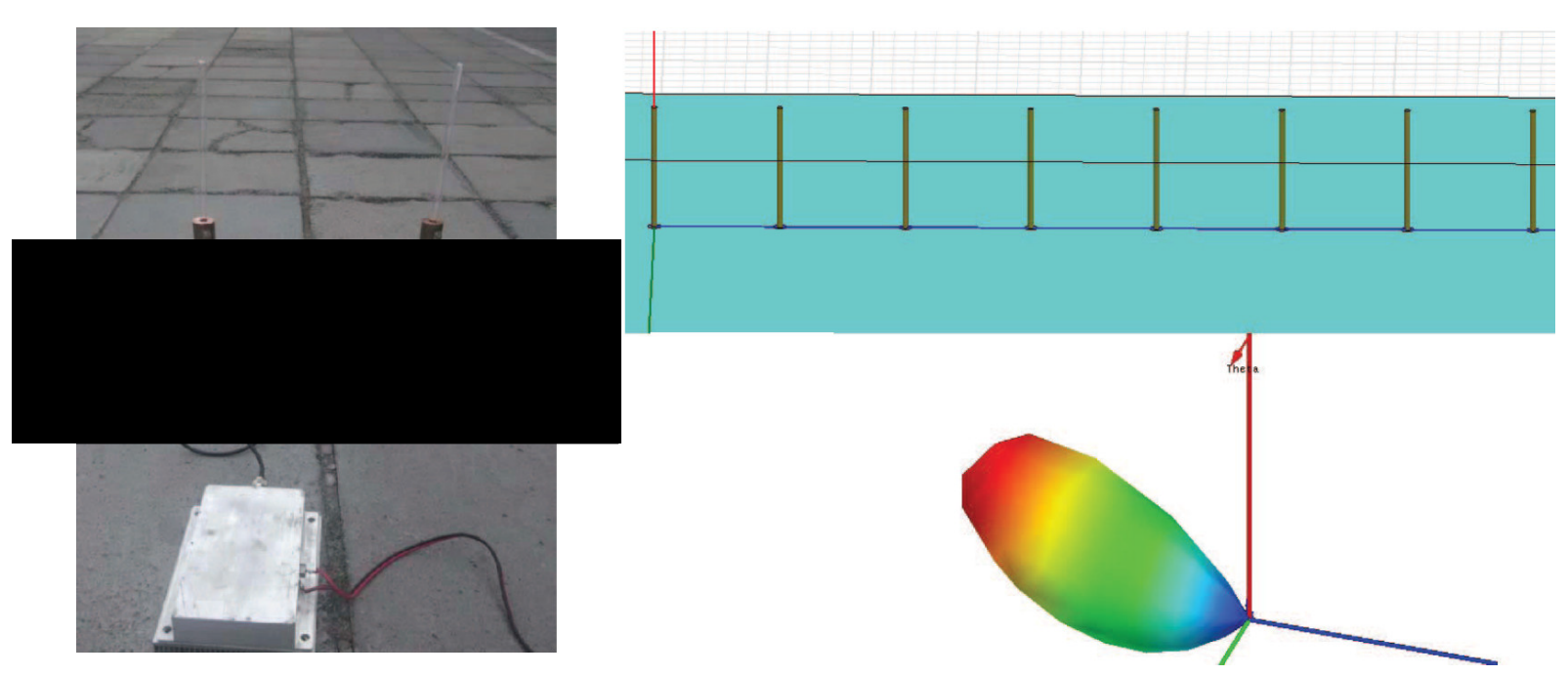

Fig. 3.29 Reconfigurable monopole plasma antenna array prototype (Left) by Sun et al. (2016) and the simulated $1 \times 8$ array results (Right).

Sun et al. (2016) has reported experimental results on a monopole antenna array made from plasma tubes. The array consisting of plasma tubes with different gaseous discharge lengths combine to form an array as shown in Fig. 3.29 (Left). This $1 \times 8$ plasma antenna array results are shown in Fig. 3.29 (Right) provides a directive reconfigurable beam pattern.

Although the work presented on plasma reconfigurable antennas thus far are promising, the reconfigurability of plasma antennas are mostly limited to changing the discharge gas column length and in varying the plasma gas density inside the tube. They are also rigid and bulky structures and are not unobtrusive. Therefore, in their present state, they are not suitable for body worn and beam steerable antenna applications and more work is needed in adapting them for such applications.

\subsection{Conformal helmet antennas}

In section 2.4 the specific requirements for this research work were specified. Among them was the requirement to build a conformal body wearable pattern reconfigurable antenna for military applications. Hence, I now look at the previous published work on helmet antennas and the work done in making conformal helmet mounted antennas.

A helmet mounted GPS antenna was presented by Fortinberry et al. (2005). This antenna has a ground plane at the bottom surface of the substrate as well as on the 4 sides of the square substrate. It has a rectangular patch antenna $(4.15 \mathrm{~cm} \times 5.45 \mathrm{~cm})$ resonating at 1.228 $\mathrm{GHz}$ and $1.575 \mathrm{GHz}$. The simulated 3D model that is presented simply places the rectangular patch antenna with the substrate, mounted on the helmet's top surface. This antenna is not 


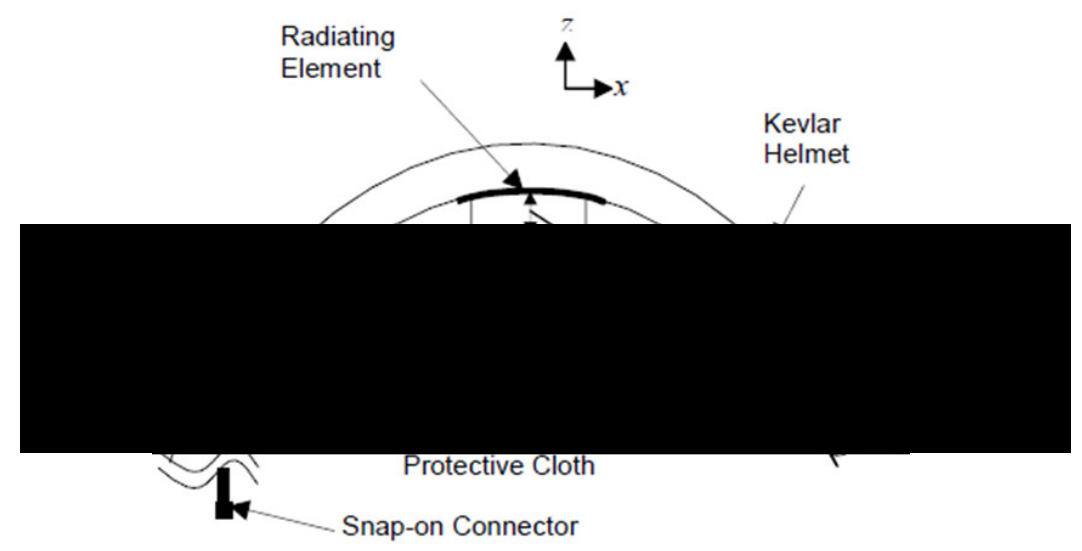

Fig. 3.30 Inside helmet antenna by Wang (2006).

conformal and its profile including the substrate protrudes above the conformal surface of the helmet and hence, the antenna is obtrusive.

Wang (2006) has presented a broadband helmet antenna mounted inside the helmet's shell. This design is shown in Fig. 3.30. It consists of the spiral mode microstrip (SMM) antenna by Wang and Tripp (1991) which is fixed conformal to the inside top surface of the Kevlar combat helmet. It has a conducting shield cloth under the antenna and its feeding network, to protect the user's head from EM radiation. It has a broadband frequency range from $1.35 \mathrm{GHz}-2.7 \mathrm{GHz}$. The reported simulated results show that it has a monopole-like pattern with a null at the zenith (Z-axis). This antenna is a near omni-directional antenna but does not have beam steerable capability for multipath mitigation or jamming avoidance etc. Wang and Triplett (2007) has also presented a much wider bandwidth (600 MHz - 4 $\mathrm{GHz}$ ). Similar to the antenna reported by Wang (2006), this antenna is connected to the inner top surface of the helmet. It consists of a conformal spiral antenna inspired by Wang and Tillery (2000) and a shielding cloth as mentioned before. At lower frequencies (300 MHz to $1.45 \mathrm{GHz}$ ) it has a near omni-directional pattern. However at the higher end of the frequency bandwidth $(>3 \mathrm{GHz})$, the beam pattern shape tends to deteriorate.

Park et al. (2007) has presented a half wavelength circular loop antenna mounted on a helmet visor. This antenna is optimized for $449 \mathrm{MHz}$ and is conformal to the helmet's visor. In order to fit in the limited space (width) of the visor, the empty space inside the half wavelength loop has been utilized to create a meander line inside it. It has an omni-directional pattern in the Z-Y plane. However, this antenna does neither have a directive pattern nor it is pattern reconfigurable.

The helmet antenna presented by Lebaric and Tan (2000b) is an ultra-wideband vertically polarized antenna consisting of two equal sized conducting strips on the camouflage cover of the combat helmet. They are connected by a thin copper strip. The top strip of the antenna is 
fed using a coaxial feed while the lower strip acts as the ground. The conducting cloth which is the substrate is made using thin polyester cloth interwoven with nickel or copper fibres, which act as the conducting strips. This antenna is conformal and can be easily integrated into the helmet using the camouflage cloth. This antenna is designed for a bandwidth from $500 \mathrm{MHz}$ to $2 \mathrm{GHz}$ (except at $900 \mathrm{MHz}$ where there is high input impedance). It has near omni-directional patterns at the higher end of this frequency range. It is lightweight and inobtrusive. Lebaric and Tan (2000a) also has reported a similar conformal helmet antenna to the one reported in Lebaric and Tan (2000b).

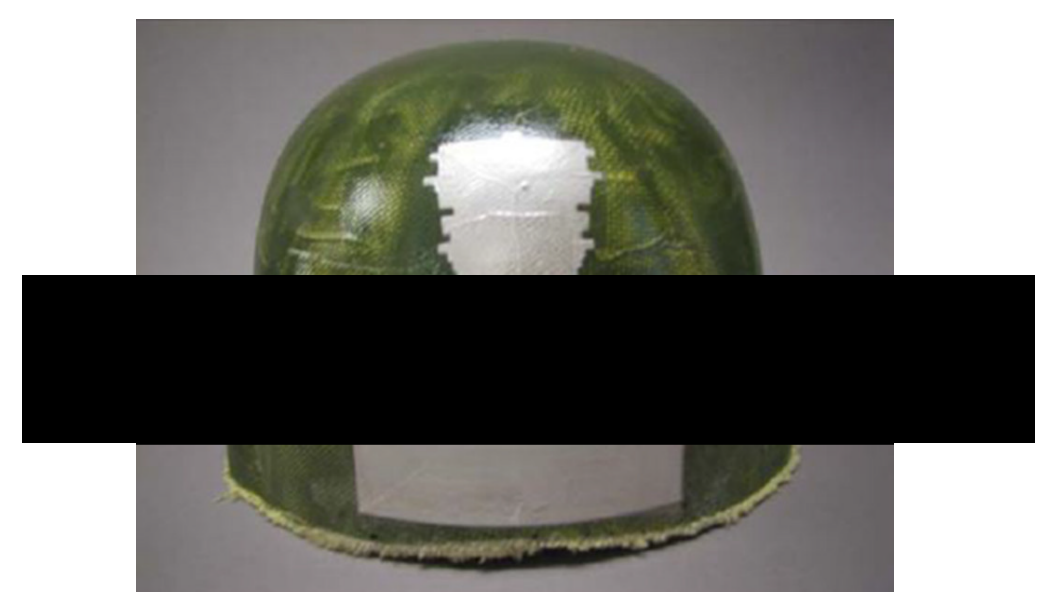

Fig. 3.31 Helmet patch antenna prototype by Herold et al. (2007).

Fig. 3.31 shows the helmet antenna by Herold et al. (2007). It consists of a conformal variable step width patch on the helmet's rear surface. It is fed with a conformal feedline over a ground patch. It is designed for $750 \mathrm{MHz}-2.7 \mathrm{GHz}$. However, no radiation pattern information is provided in the work.

\subsection{Beam switching algorithms}

In the directive pattern reconfigurable antenna discussed in the previous sections, the implementation of a beam switching/steering algorithm is necessary to point the antenna's beam to the direction of the LOS signal or the strongest signal path. These algorithms play a critical role in the mitigation of multipath, jamming avoidance or user tracking applications discussed in Section 2.4. Several signal processing and beam switching algorithms have been discussed in literature in this area. Combining such a beam switching algorithm with a directive pattern reconfigurable antenna system provides a robust communication system that can solve the issues in the application discussed in Section 2.4. 


\subsection{Beam switching algorithms}

In the past, antenna arrays have mainly been used for signal scanning applications using beam steering algorithms. DoA estimation algorithms utilising DSP techniques were the preferred method for such antenna arrays. However, there are very few reported work that utilises single unit pattern reconfigurable antennas for implementing beam switching/scanning algorithms.

In this section, I look at several reported work that discussed beam switching/scanning algorithms that can be applied for single unit pattern reconfigurable antennas (not antenna arrays), keeping in mind the limited spatial beam switching capabilities of single unit pattern reconfigurable antennas previously discussed.

Sanchis et al. (2002) proposes an algorithm based on power measurements of pilot tones similar to the mono-pulse technique used for DoA estimation in radar systems. In the proposed method, a mobile subscriber sends short duration tone to the mobile base station (BS). The BS measures the instantaneous signal power using 3 beam positions. The measured power, $P_{i-1}, P_{i}$ and $\left.P_{i+1}\right)$ corresponds to the previous, current and adjacent position's of the moving mobile subscriber. It then computes the the tracking estimator, $\mathrm{d}$ as follows:

$$
d=\frac{P_{i+1}-P_{i-1}}{P_{i}}
$$

If the estimation $d$ is near zero, then the mobile subscriber is moving within the current position. If $\mathrm{d}>0$, then the user is moving to the adjacent position while $\mathrm{d}<0$ means the user is moving back to its previous position. This type of algorithm requires 3 beam positions and constant/periodic scanning to track the movement of the mobile subscriber. The short duration tones sent prior to data transmission also adds a small overhead cost.

A selection combining (SC) scheme is a multi receiver (or multi beam) system where it chooses to switch to the antenna (or beam) with the largest signal amplitude among its multiple receivers (or beams), always switching to the stronger branch (Zhao et al., 2015). According to Beaulieu (2008), a two antenna receiver SC scheme forms the random process defined as:

$$
Z(t)=R_{1}(t)-R_{2}(t)
$$

where $\mathrm{Z}(\mathrm{t})$ is the random variable which is used as the switching metric and $R_{1}(t)$ and $R_{2}(t)$ are the instantaneous signal amplitudes of receiver 1 and receiver 2 respectively. Zero crossing of $Z(t)$ from negative to positive means that the scheme switched from $R_{2}(t)$ to $R_{1}(t)$ and zero crossing of $Z(t)$ from positive to negative means it switched from $R_{1}(t)$ to $R_{2}(t)$. 
A switch-and-stay-combining (SSC) scheme algorithm monitors a switching criteria to decide on whether to switch to a different antenna/beam direction. Once switched, the beam stays in that direction. A switch-and-examine-combining (SEC) scheme will continue to monitor the switching criteria in the new position. When the beam switching criteria is met again, it switches to the next direction (Moradi et al., 2012). A common method used in SSC and SEC switching criteria schemes is to monitor the signal-to-noise-ratio (SNR) and switch to another beam direction when the SNR drops below a pre-agreed threshold.

Chau and Chen (2010) proposes a SSC scheme which uses the fading factor $\alpha$ of the channel and the matched-filter output of the receiver $\boldsymbol{r}$ as metrics as the switching criteria instead of the traditional SNR based SSC scheme. This work also provides a closed form derivation for the globally optimal switch threshold.

The rate of switching between two antenna beams based on the chosen SSC scheme is an important factor. Jataprolu et al. (2011) says that switching to the other beam is followed by an idle time intervals. This is similar to an internal outage at the receiver. Therefore, beam switching will become an issue for signal reception if the frequency of occurrence of beam switching increases. Furthermore, efficient channel estimation requires that the antenna beam is fixed for a certain period. Therefore, beam switching before the estimation is complete will also create errors in channel estimation. (Jataprolu et al., 2011) discusses in detail the optimal switching threshold that will lead to the optimal bit error rate (BER) performance of SSC scheme, when the switching rate is constrained. However, negative effects of non-optimal beam switching frequency such as channel estimation errors does not apply for the problem statement discussed in Section 2.4.

The switching threshold value also plays an important role in the BER performance of a SEC scheme. When the threshold value is set higher it increases the processing load as the antenna system will then be prone to constant switching when the SNR value drops below this threshold. If it is set too low, then optimal switching may not occur, resulting in BER deterioration. Therefore, the threshold level selection for SEC is a trade-off between BER performance and receiver complexity as mentioned by Moradi et al. (2012).

\subsection{Conclusion}

In this chapter I first defined reconfigurable antennas and then looked at how reconfigurable antennas have evolved over the past. Due to the nature of the problem statement discussed in Section 2.4, I selected mainly single unit pattern reconfigurable antennas for detailed study. In the next section, I looked at various single unit pattern reconfigurable antennas and the techniques used to switch patterns in them. 
During the review of published work, it was observed that the majority of the pattern reconfigurable antennas used p-i-n diodes, RF switches, FET switches and varactors as the preferred method for switching the surface current path to different radiating elements/parts of the antenna. Most planar pattern reconfigurable antennas studied carried more than one radiating element, separated by these switches. The status of the switches (ON/OFF) determined the current path along the antenna and hence, gave rise to different radiation patterns for different switching configurations.

FSS were used to create directive antenna beams and steer them in the azimuth direction, to cover a wider beam scan range. By switching ON/OFF certain columns of cells of the FSS, they can be made to appear transparent or opaque to EM waves incident on them. This results in a steerable directive antenna. The disadvantage of the FSS is their 3-dimensional nature and the large bulky size.

Pattern reconfigurable antennas using HIS structures were next discussed in Section 3.5. HIS using structures with vias have a complex rigid design. Therefore, they are not ideal for conformal applications. However, a few examples where conformal antennas arrays were made using conformal HIS structures were discussed. These designs did not have vias to connect the patches to the ground. Antennas using HIS have low profile height which is an advantage in the design of unobtrusive conformal antennas for helmets due to their low visible signature.

The next section studied pattern reconfigurable antennas with non-planar (3D designs). Some of the work studied included folded dipoles in a 3D structure. Due to their abstract shape, they are not ideal for the conformal or body wearable applications.

I then looked at the planar SLA designs. These antennas have a 4-feed system to generate 4 identical beams. The beams generated when each port is switched ON, is a tilted directive pattern pointing to one quadrant in space at a time. Two feeding networks to conduct the beam switching were discussed. This antenna has the ability to generate a CP axial beam as well as a semi-doughnut type pattern when all 4 ports are fed simultaneously with the appropriate phase differences. By switching between the 4 ports, the beam direction can be changed, thereby covering $360^{\circ}$ Azimuth range. Later reported work have proposed the use of dual frequency bands using dual square loops. Capacitive feeding had been introduced for the SLA as well as the use of HIS to reduce the antenna profile height. Due to its 4 identical beams and the ability to produce a near-omni like pattern along with the ability to steer the beam in the azimuth direction, this antenna is a good candidate as a possible solution for the problem statement discussed in Section 2.4.

I next studied the reported work in making reconfigurable antennas conformal. Some early work involved placing components such as p-i-n diodes and RF switches on the conformal 
surface of the antennas, which is not ideal. Later work involved the development of antennas using textile fabric as a substrate. Most of the reported work involved single unit antennas with little or no pattern diversity, except the antenna presented by Jais et al. (2013) which has 4 directive beam patterns in the 4 quadrants in space. The beam switching mechanism was implemented using p-i-n diodes. For conformal applications on a more rigid substrate, the applications of diodes as switching elements and control wires running across srufaces need to be reconsidered. Damis et al. (2016) also has proposed a flexible square loop antenna which has the potential to generate a reconfigurable pattern but more work is needed in studying the effects of feeding at different points along the loop.

I have then discussed some of the latest developments in the field of reconfigurable antennas using plasma antennas. They use an ionized plasma contained in a rigid tube which is excited. Several properties such as the gaseous discharge length and the plasma density can be varied in reconfiguring the antenna radiation beam pattern.

The penultimate section reviewed some of the work done in designing helmet antenna prototypes. It was noted that nearly all the work presented thus far carried non-pattern reconfigurable antennas conformal to the helmet surface. However, a gap in knowledge was identified where a pattern reconfigurable conformal antenna can be designed using some of the designs discussed above.

Finally, I reviewed the three major combining schemes: SC, SSC and SEC. It was noted that the selection of an optimal switching frequency and a optimal switching threshold for the switching metric plays a crucial role in the BER performance of each scheme. The exact algorithm and the beam switching scheme to be implemented in this research work will largely depend on the available hardware and processing power and the nature of the test environment. 


\section{Chapter 4}

\section{Conformal 2.4 GHz beam steering antenna for high throughput applications in weak EM environments}

\subsection{Introduction}

In this chapter, I present a beam adaptive communication system for sensor networks with increased throughput. In this system, the antenna is a conformal four port square loop antenna. It is printed on a hemispherical substrate and has a perimeter of length $5.6 \lambda_{g}$. The antenna can generate four titled beams of gain $8.94 \mathrm{dBi}$ in four space quadrants, depending upon which of the four feeds (ports) is excited. To direct power to a selected port, the system employs an SP4T RF switch operating under a microcontroller. With the aid of a Bluetooth link the controller is linked with a browsing roaming device (a laptop) running an autonomous algorithm in $\mathrm{C \#}$ language.

Under an algorithm the router scans fast using the switch and locks to one of the four beams with the highest RSSI (Receive Signal Strength Indicator). This adaptive process is initiated every time the signal drops by $3 \mathrm{~dB}$ or below a threshold. For a fixed spectrum in a weak electromagnetic environment the adaptive beam steering antenna enables a 70-fold throughput jump (7.5 Mbps jump from 0.1 Mbps) in the system compared to when the beam switching algorithm is not active. For the remainder of this chapter, a weak electromagnetic environment is defined as an electromagnetic environment where the received signal strength is close to the noise floor in that environment. 


\section{Conformal 2.4 GHz beam steering antenna for high throughput applications in weak EM environments}

\subsection{Background}

The application of randomly distributed sensors over a large area is of interest to researchers and organizations involved in monitoring natural phenomena (Chen et al., 2013), large scale safety systems (Giri and Wall, 2008) and military (Song et al., 2009). By loading them with a weight, spherical shells in particular can be balanced in a specific direction and for this reason they are a good option for accommodating sensors and the associated transceiver. Each sphere is desirable to be equipped with a simple antenna capable of adapting its beam to maximize the signal to noise at the monitoring location. Ideally the antenna is conformal and printable on the surface of the sphere.

The single loop antenna (SLA) with ground plane is little disturbed by capacitive effects of the environment and hence is a good candidate for sensor networks. It is widely used in various communication systems. In planar form, it is simple to design, easy to tune, low cost to manufacture, and can offer switched beam steering capability (Deo et al., 2010; Mehta and Mirshekar-Syahkal, 2007; Pal et al., 2014c, 2008, 2012b). The loop antenna has emerged in various shapes including circular, square and triangular.

Furthermore, the SLA with ground has been reported to be appropriate for multi-beam using multi-feed technique, in which case it can generate a beam with a direction controlled by a switch. For example, Deo et al. (2011b) presents a four-feed 4.5 GHz SLA integrated with a Single-Pole-4-Throw (SP4T) for directing power to one of the four ports of the antenna, generates a tilted beam switchable to each of the 4 quadrants of the space.

Also, for the same antenna with all its four ports excited, the possibility of the generation of a semi-doughnut and axial pattern for vehicular and wireless applications has been studied in Pal et al. (2013a, 2011). In Pal et al. (2017) it is also demonstrated that the array of square loop antennas can significantly improve the scanning range compared to conventional patch antenna array.

\subsection{System design}

\subsubsection{Conformal beam steerable antenna on a hemisphere}

In this chapter a switched beam conformal SLA with ground plane on a hemispherical surface, as shown in Fig. 4.1, is proposed and studied. The conformal beam steering antenna developed in this work has long perimeter, $\mathrm{C}=384 \mathrm{~mm}=3.1 \lambda_{0}$ at $2.45 \mathrm{GHz}$. It is printed on a hemispherical shell substrate of outer radius $187 \mathrm{~mm}$ and it produces a tilted beam of directivity $8.94 \mathrm{dBi}$. 


\section{Windows 8}

laptop with

built-in WiFi

antenna

WiFi
connection

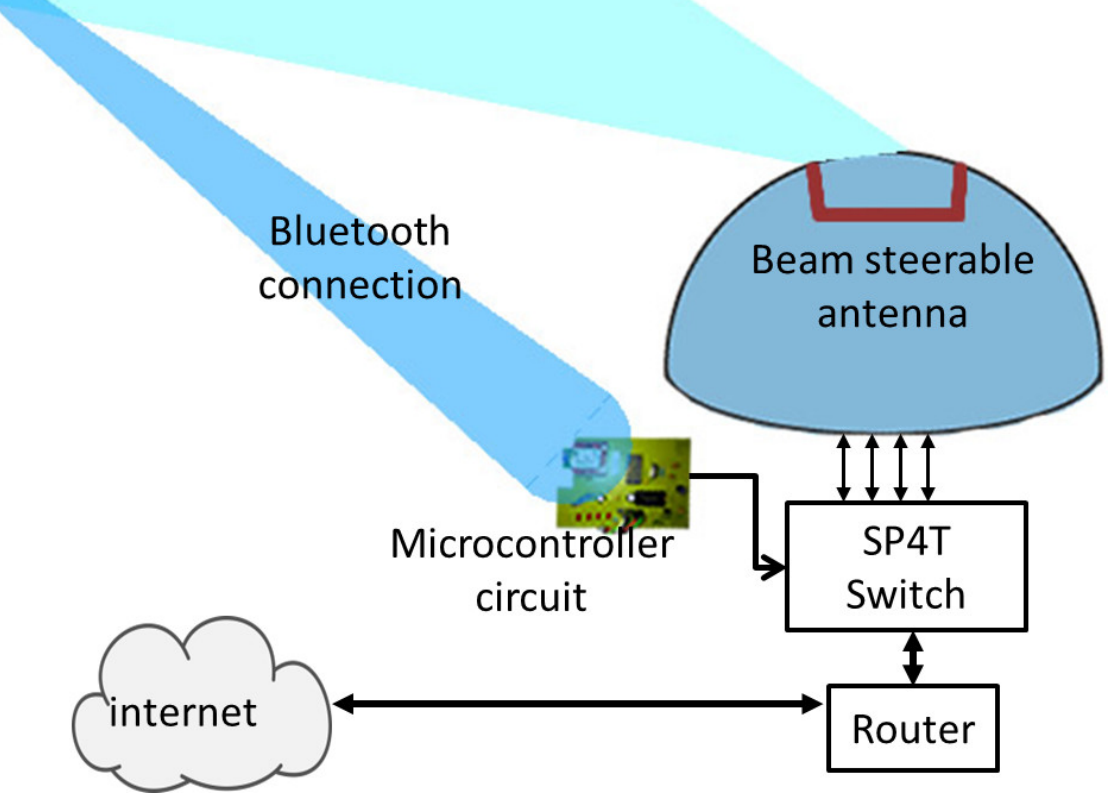

Fig. 4.1 The conformal SLA on spherical surface and its integration with WiFi router system for throughput enhancement. 
A demonstration for this beam switching in the 4 quadrants in space using an automatically controlled SP4T PIN diode switch and a control circuit is shown in this chapter. The simulated and experimental radiation patterns and return losses of the antenna under changing of various parameters (substrate thickness, width, bending etc.) are also reported. All of the previous work published in this area (Deo et al., 2010; Mehta and Mirshekar-Syahkal, 2007; Pal et al., 2014c, 2008, 2012b) were on a planner structure with much smaller perimeter lengths.

\subsubsection{Integration of conformal antenna with $2.4 \mathrm{GHz}$ WiFi router}

To demonstrate the effect of the beam switching on the throughput of a communication system the antenna is integrated with a $2.4 \mathrm{GHz}$ WiFi router, shown in Fig. 4.1. The results of this investigation is important as in a practical network with randomly distributed sensors, the locations of the receivers (or the relaying nodes) and the sensors may change with time and hence the beam from the sensors will need realignment to maintain high throughput communications.

As explained further in this chapter, throughput enhancement of the system is achieved using the combination of two beam switching techniques, switch-and-stay and switch-andexamine schemes, as mentioned in Sanford (1978).

\subsection{Antenna configuration and results}

\subsubsection{Hemispherical square loop antenna (HSLA)}

Fig. 4.2 shows the top, perspective, bottom and cross-sectional views of the SLA with ground on a hemispherical shell for $2.4-2.5 \mathrm{GHz}$ band. In this chapter, this antenna configuration is referred to as 'HSLA'. Its shell is made from Kevlar, has an outer radius, $c=a=175 \mathrm{~mm}$ and has a thickness $h_{1}=8.5 \mathrm{~mm}$. It has a relative permittivity, $\varepsilon_{r 1}=3.4$.

The conformal square loop consists of 4 copper strips of width $\mathrm{t}=4 \mathrm{~mm}$ and length, $W_{a}=96 \mathrm{~mm}$. They are sited on the outer top surface of the hemisphere with the center of the loop on the Z-axis. A Rohacell ${ }^{\circledR} 51$ foam spacer $\left(\varepsilon_{r 2}=1.04\right)$ of thickness, $h_{2}=14 \mathrm{~mm}$ is fixed to the bottom of the Kevlar layer for the placement of the antenna ground. This therefore makes the total antenna profile height to $22.5 \mathrm{~mm}\left(0.18 \lambda_{0}\right)$. The ground plane is conformed and fixed to the inner surface of the foam spacer as shown in Fig. 4.2(b). The conformal length of each edge of the ground plane $W_{g}=162 \mathrm{~mm}$.

The 4 radiating arms of the square loop are fed at their centers using conducting pins of $1.3 \mathrm{~mm}$ diameter which are connected to 4 SMA connectors (ports) A, B, C and D. Similar to 


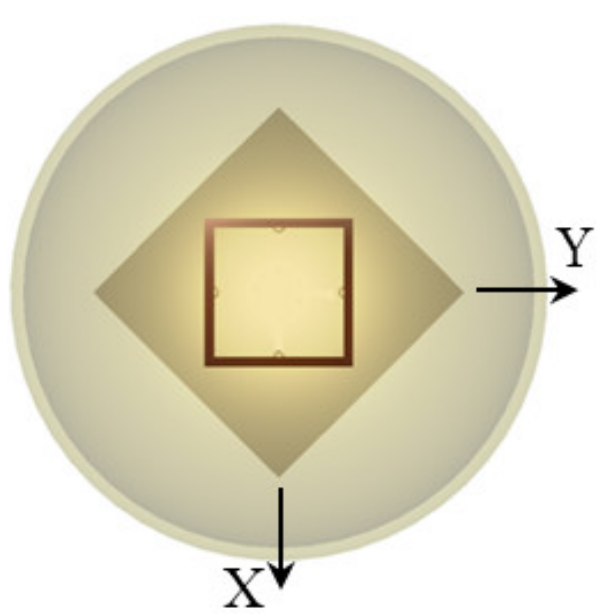

(a)

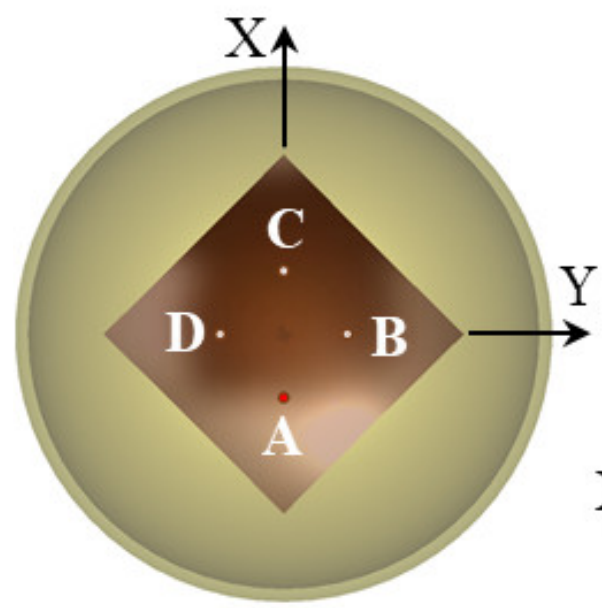

(c)

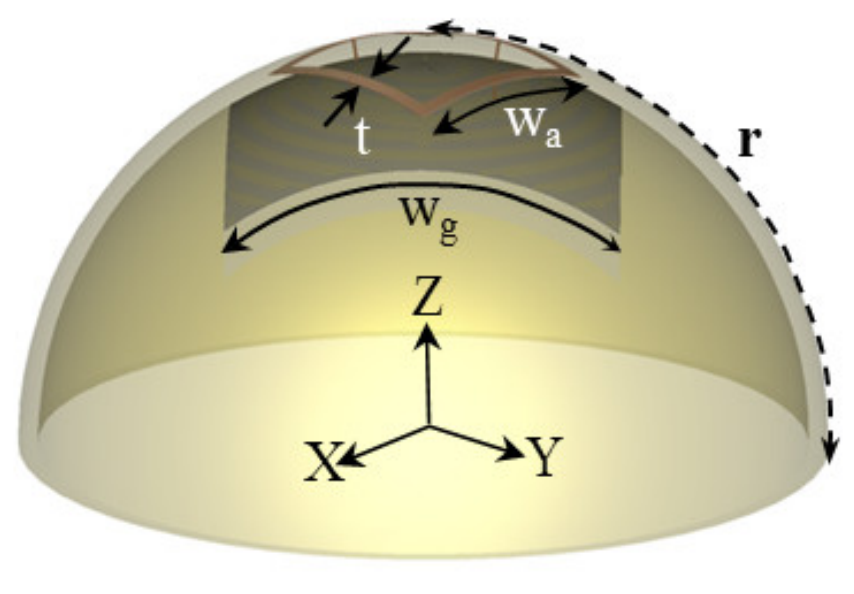

(b)

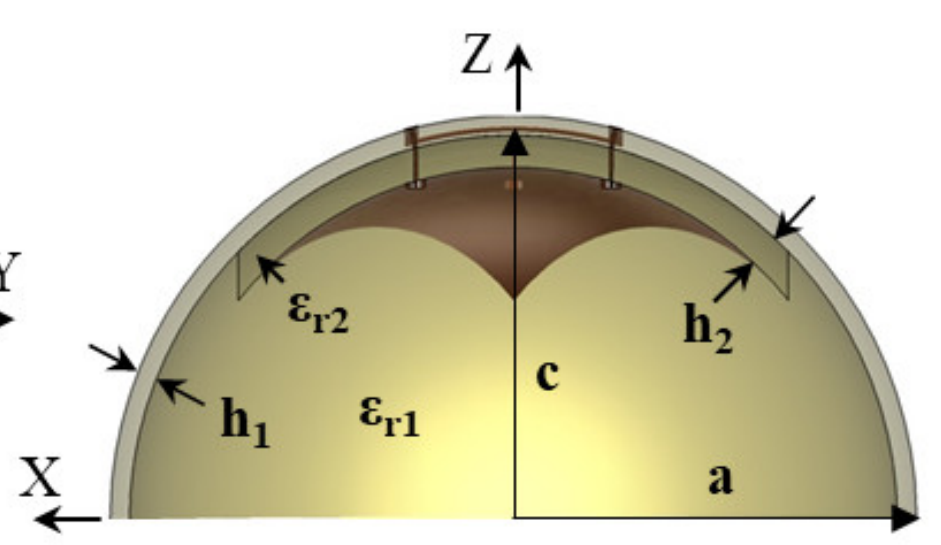

(d)

Fig. 4.2 The perspective, bottom and cress-sectional views of the HSLA 


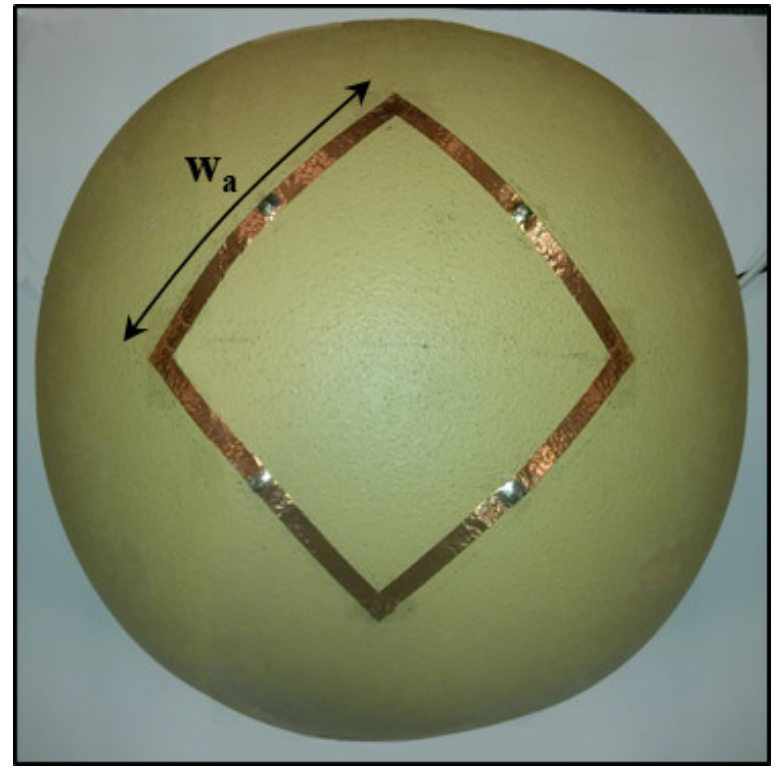

(a) Top view
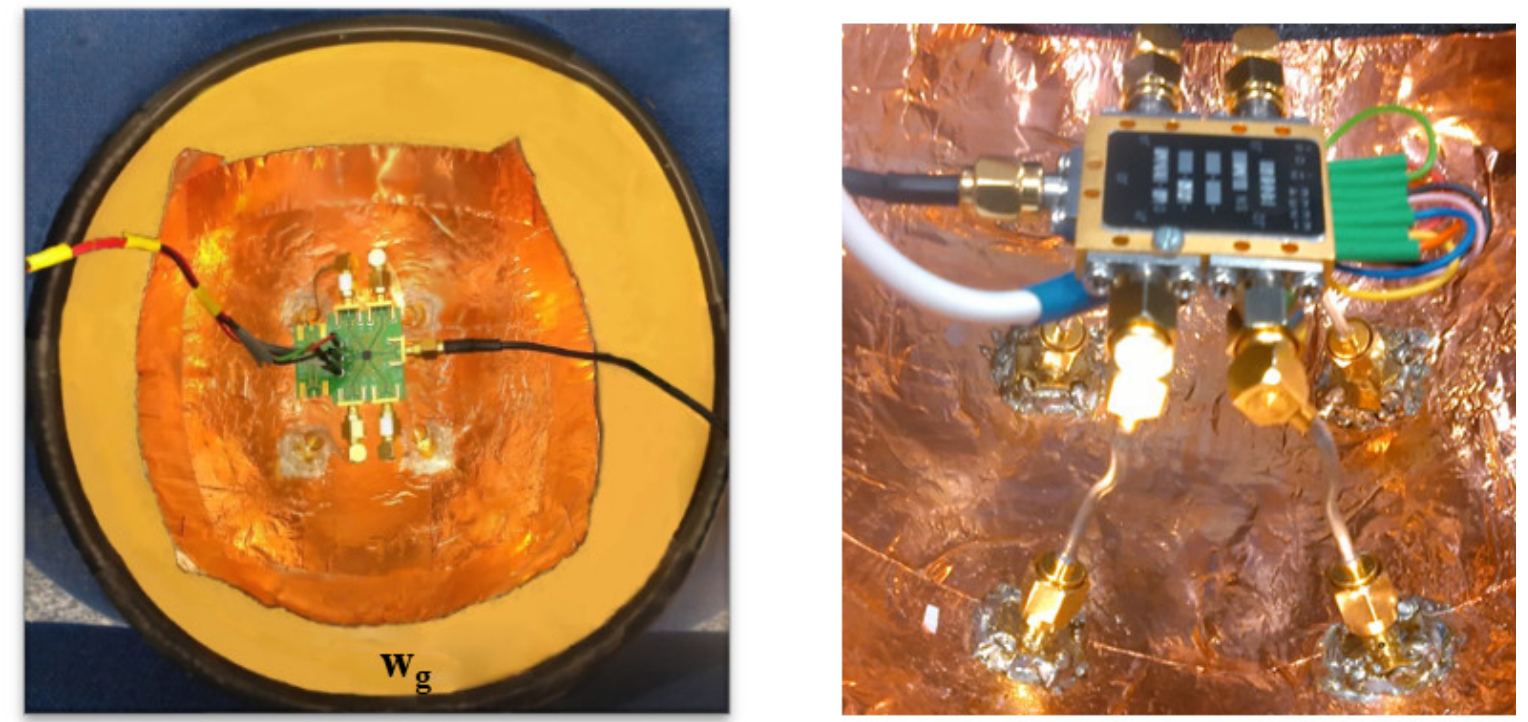

(b) Bottom view (Left) and the electronic switch (Right)

Fig. 4.3 Fabricated HSLA on a hemispherical shell (a) Top view (b) Bottom view and the electronic switch. 
(Deo et al., 2010; Mehta and Mirshekar-Syahkal, 2007; Pal et al., 2014c, 2008, 2012b), only one port is excited at a time. When one port is fed the remaining 3 ports are open circuited using a RF switch (Devices, 2009). The fabricated HSLA is shown in Fig. 4.3.

\subsubsection{Simulated and experimental results}

The plots of return loss coefficient $\left(\left|S_{11}\right|\right)$ (in $\mathrm{dB}$ ) against frequency for the computational and experimental models of the HSLA antenna are presented in Fig. 4.4a, indicating good agreement. However, $\left|S_{11}\right|$ at the frequency band of interest, $2.4-2.5 \mathrm{GHz}$, is well above -10 $\mathrm{dB}$. The evaluation of the input impedance of the antenna as shown in Fig. 4.4b, shows that at $2.45 \mathrm{GHz}$ it is about $16-j 56 \Omega$. Therefore, a matching circuit was developed to match the antenna to the standard $50 \Omega$ impedance.

The matching circuit is shown in Fig. 4.5a with its equivalent circuit Fig. 4.5a(Bottom). Fig. 4.5b shows the $\left|S_{11}\right|$ against the frequency before and after applying the impedance matching. As can be seen, an impedance bandwidth of $200 \mathrm{MHz}$ in the ISM band is achieved after the matching.

Similar to Mehta and Mirshekar-Syahkal (2007), Pal et al. (2008), Deo et al. (2010), Pal et al. (2014c), Pal et al. (2012b) and Deo et al. (2011b), it was found that the HSLA produces a titled beam $\left(\theta_{\max }=46^{\circ}\right)$ of Gain $8.94 \mathrm{dBi}$ when one of its ports is excited and other three ports are left open circuited. However, because of conformal base and larger perimeter the beam tilt was highest compared to earlier recorded work in Pal et al. (2008), Deo et al. (2010) and Pal et al. (2014c). The polar plots for the radiation pattern for port A feeding, are shown in Fig. 4.6. As shown in Mehta and Mirshekar-Syahkal (2007), Pal et al. (2008), Deo et al. (2010), Pal et al. (2014c), Pal et al. (2012b) and Deo et al. (2011b), by sequentially exciting ports A to D, the HSLA can steer the $8.94 \mathrm{dBi}$ beam in full azimuth space. This is shown in Fig. 4.7.

\subsection{Parameter study}

\subsubsection{Effects of variation of separation between ground plane and ra- diating strips on the radiation pattern}

The total profile $\left(h_{1}+h_{2}\right)$ of the conformal SLA with ground on the hemisphere in the test design was chosen to be at $22.5 \mathrm{~mm}$. I next studied the effects of varying this thickness or separation between HSLA radiation strips and ground plane on the radiation pattern. The 


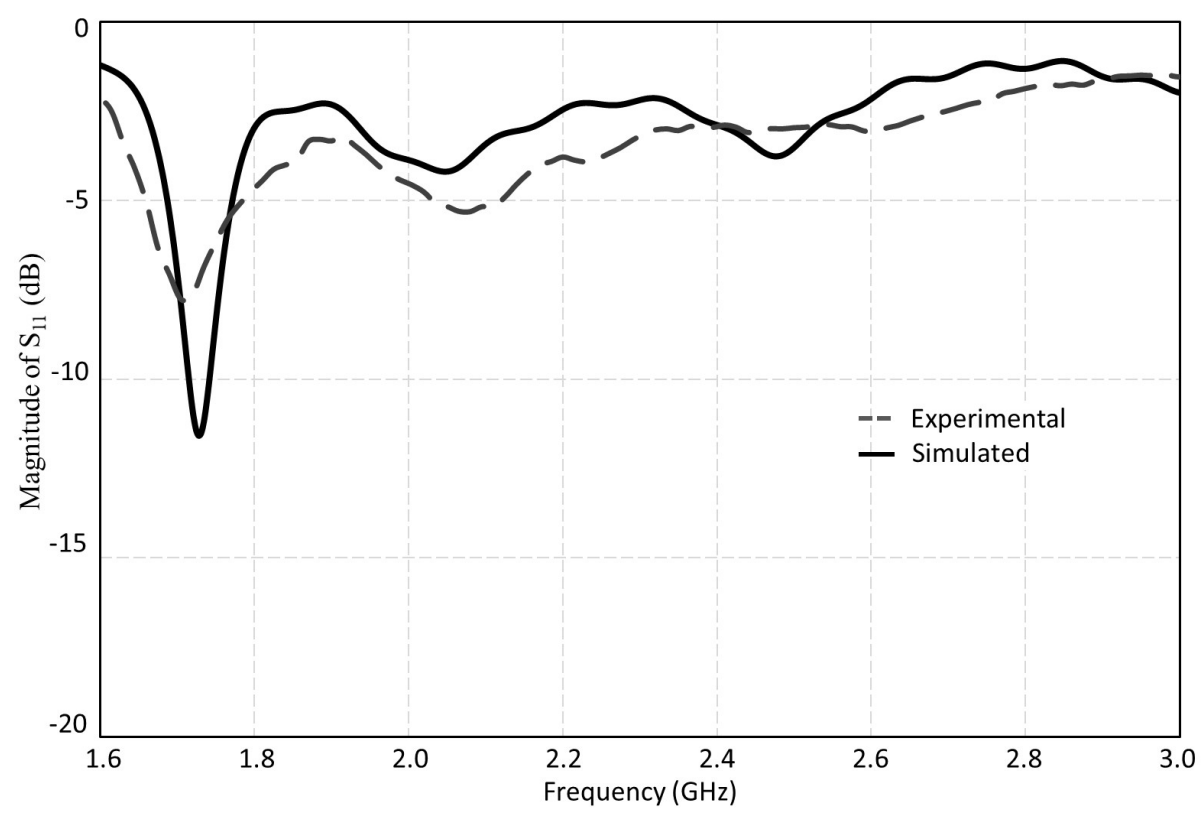

(a) Computational and experimental frequency response of reflection coefficient $\left(\left|S_{11}\right|\right)$ of HSLA

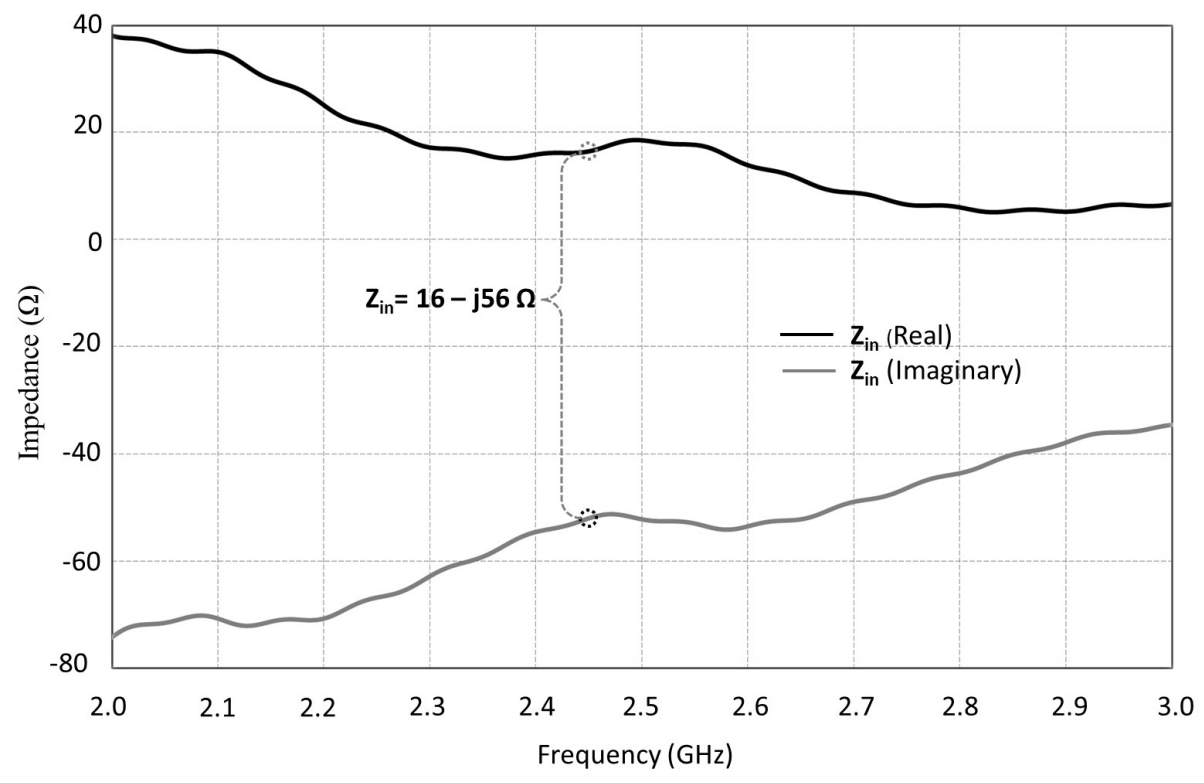

(b) Experimental frequency response of input impedance $Z_{\text {in }}(\Omega)$ of HSLA

Fig. 4.4 Frequency response of reflection coefficient $\left(\left|S_{11}\right|\right)$ of HSLA and its input impedance 

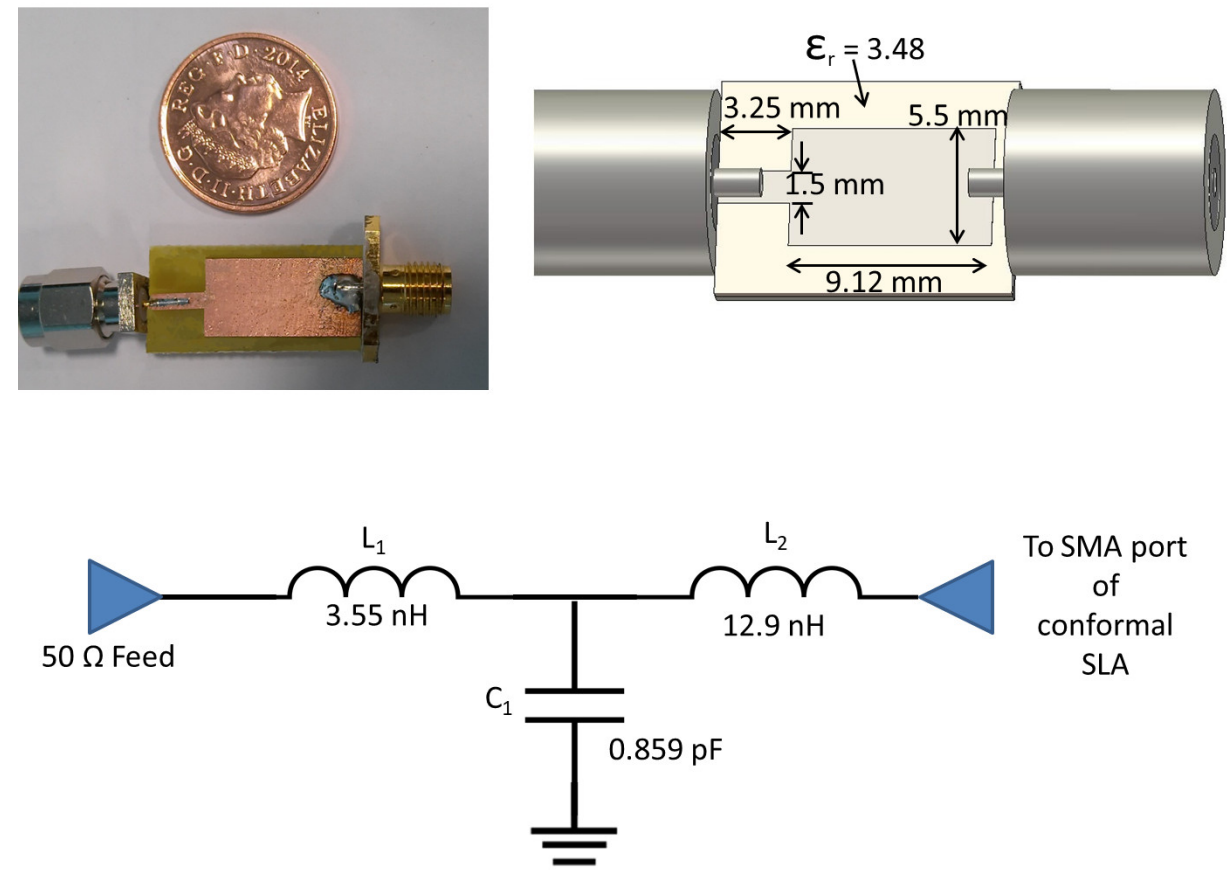

(a) Fabricated component (Top Left), simulated model of component (Top Right) for impedance matching, $\left(w_{1}=10.9 \mathrm{~mm}, w_{2}=1.75 \mathrm{~mm}, l_{1}=9.55 \mathrm{~mm}, l_{2}=1.38 \mathrm{~mm}, \varepsilon_{r}=4.5\right.$, height $\left.=1.6 \mathrm{~mm}\right)$ and its equivalent circuit (Bottom)

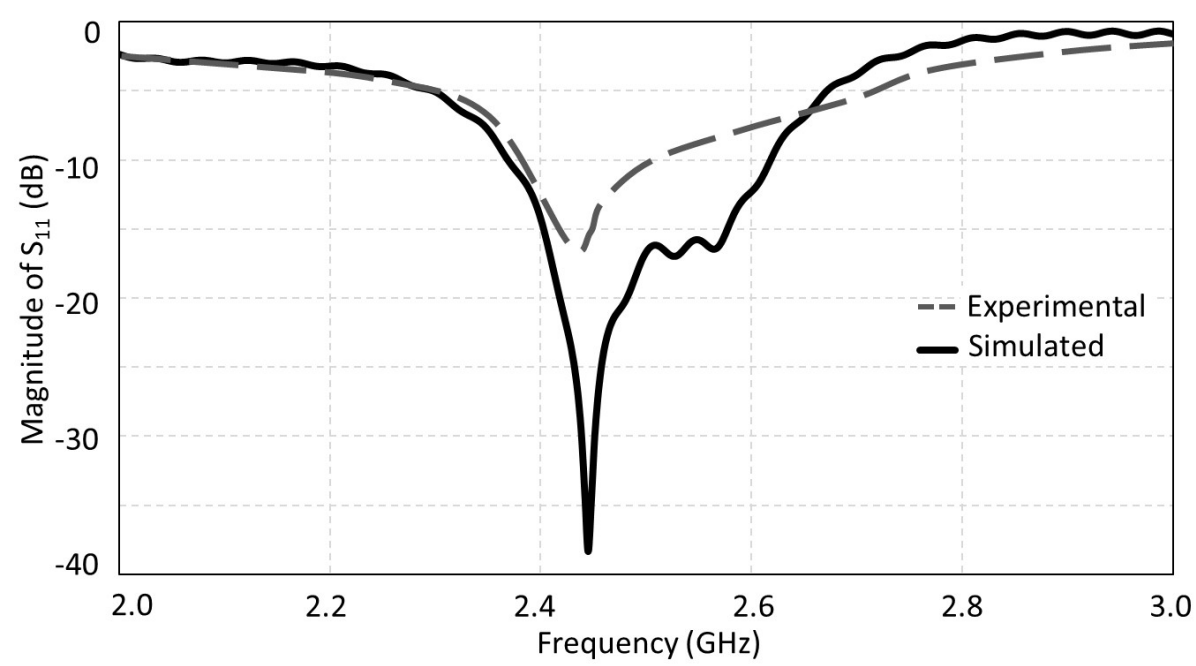

(b) $S_{11}$ after impedance matching using microstripline component

Fig. 4.5 (a)Fabricated and simulated models of component for impedance matching, its equivalent circuit and (b) $S_{11}$ of HSLA after impedance matching 


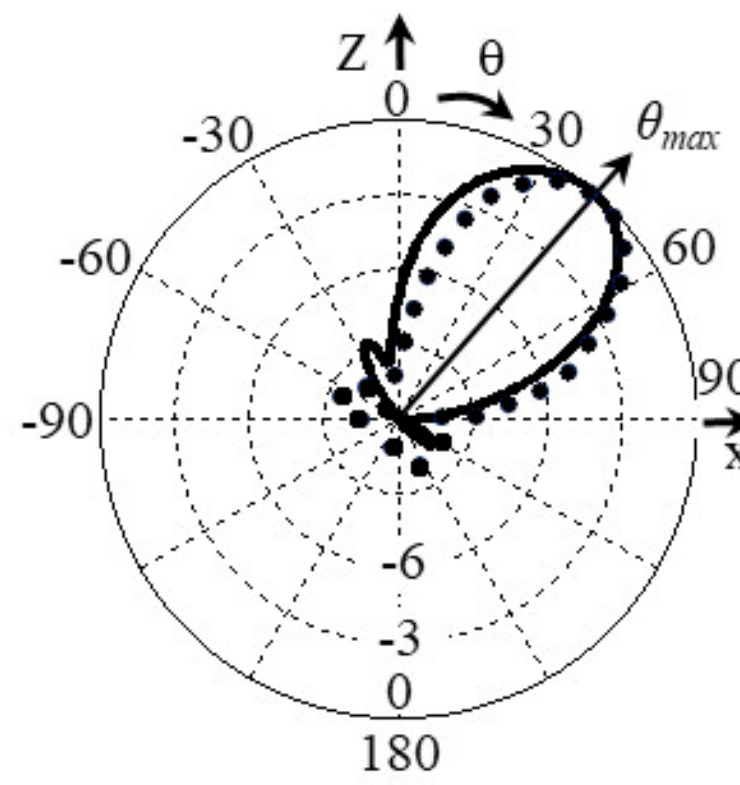

Elevation plane at $\phi_{\max }=0^{\circ}$

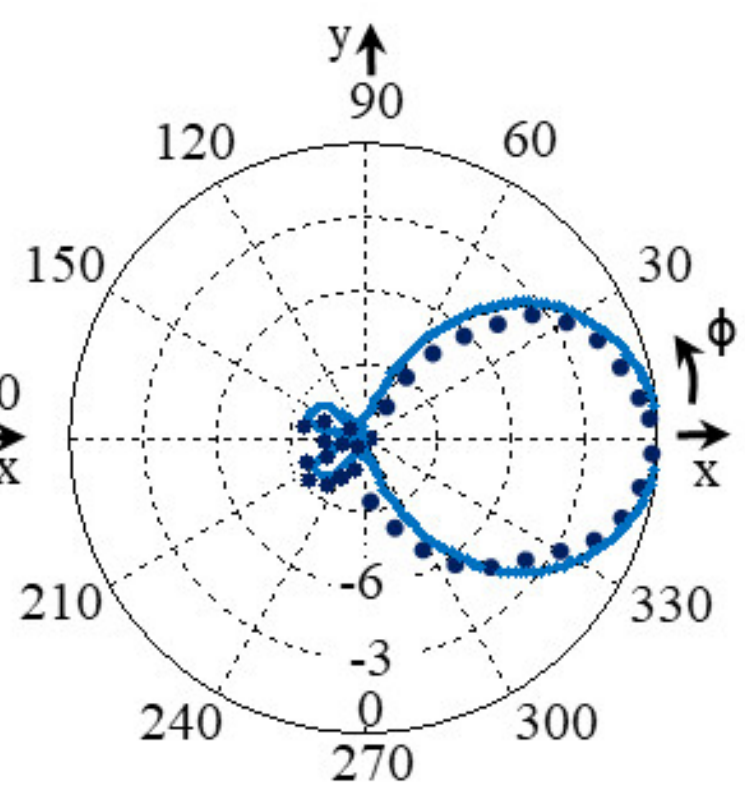

Azimuth plane at $\theta_{\max }=40^{\circ}$

$$
\begin{aligned}
& \mathrm{E}_{\theta}\{- \text { Simulated, } \cdots \text { Measured }\} \\
& \mathrm{E}_{\phi}\{- \text { Simulated, } \cdots \text { Measured }\}
\end{aligned}
$$

Fig. 4.6 Radiation pattern of HSLA: (Left) Elevation and (Right) Azimuth plane polar plots 


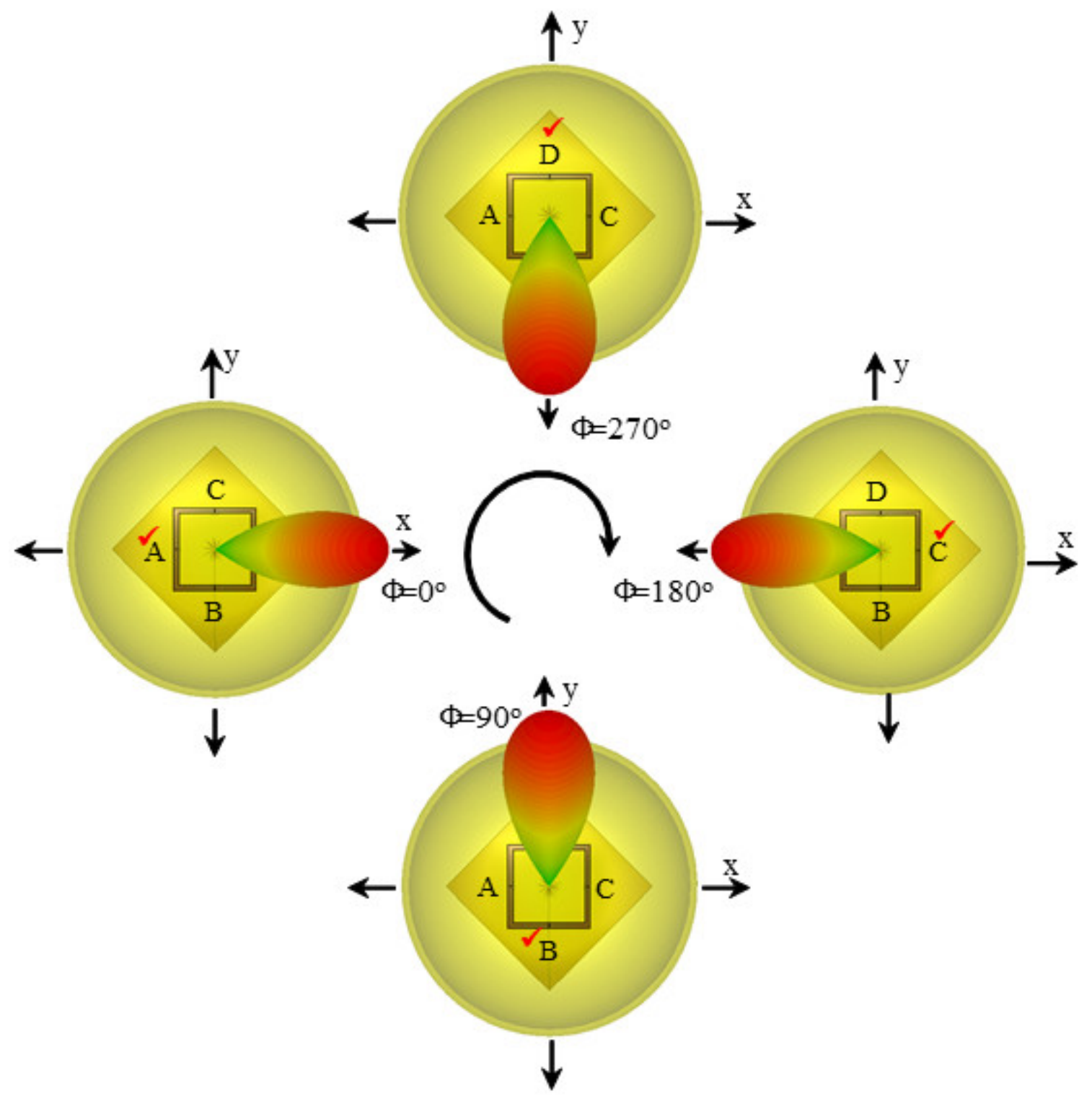

(b)

Fig. 4.7 Demonstration of beam steering using HSLA using ports A, B, C and D 

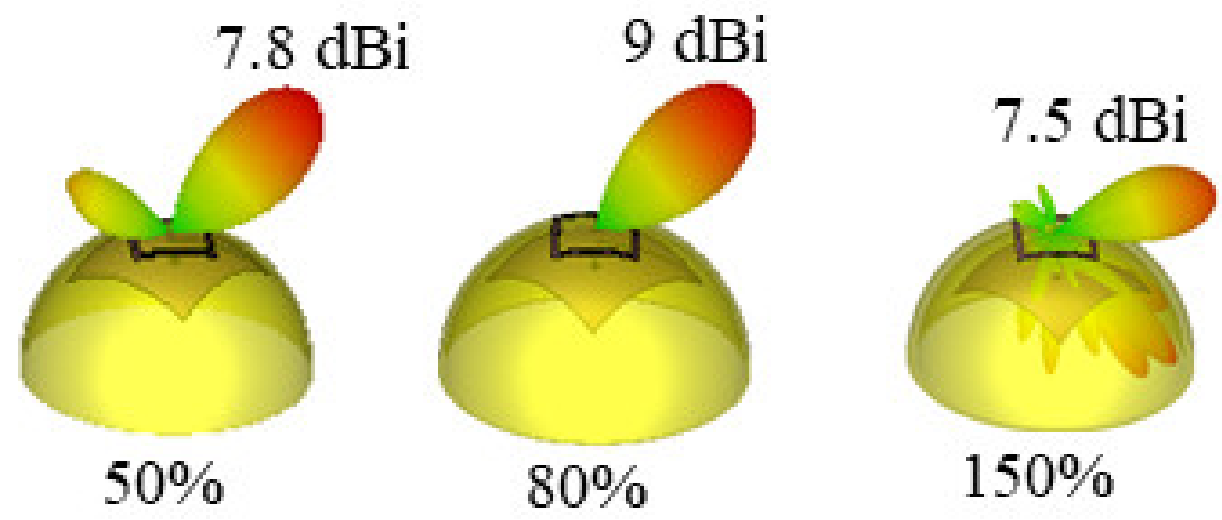

(a) The change in radiation pattern of HSLA when $h_{1}+h_{2}$ was varied from $50 \%(11.25 \mathrm{~mm})$ to $150 \%$ $(30.75 \mathrm{~mm})$

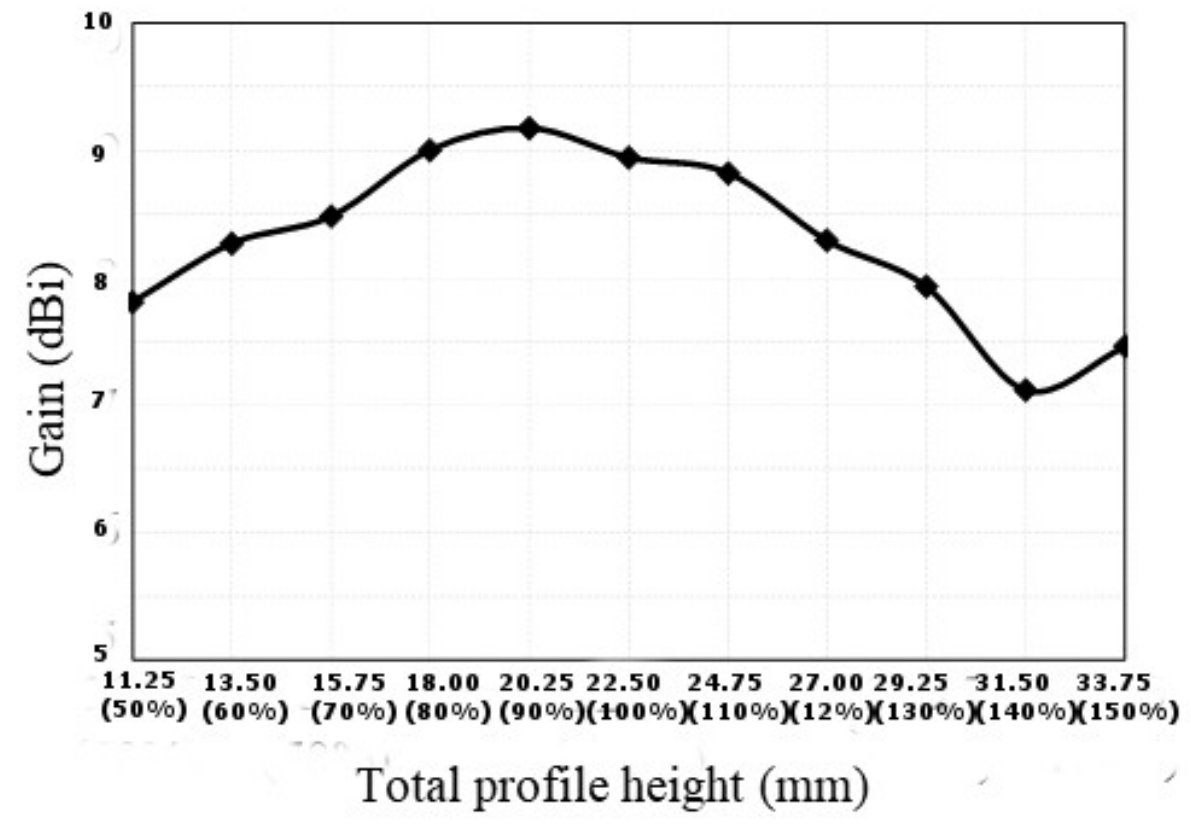

(b) The change in Gain of HSLA when $h_{1}+h_{2}$ was varied from $50 \%(11.25 \mathrm{~mm})$ to $150 \%(30.75 \mathrm{~mm})$

Fig. 4.8 The change in radiation pattern and Directivity when total profile height was varied. 


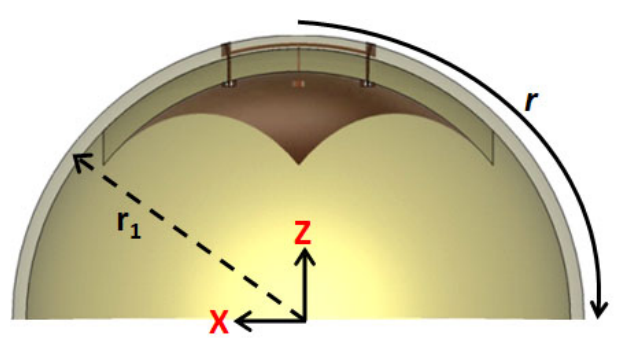

(a) The curvilinear distance $r$ is defined as the curved distance from the top to the rim.
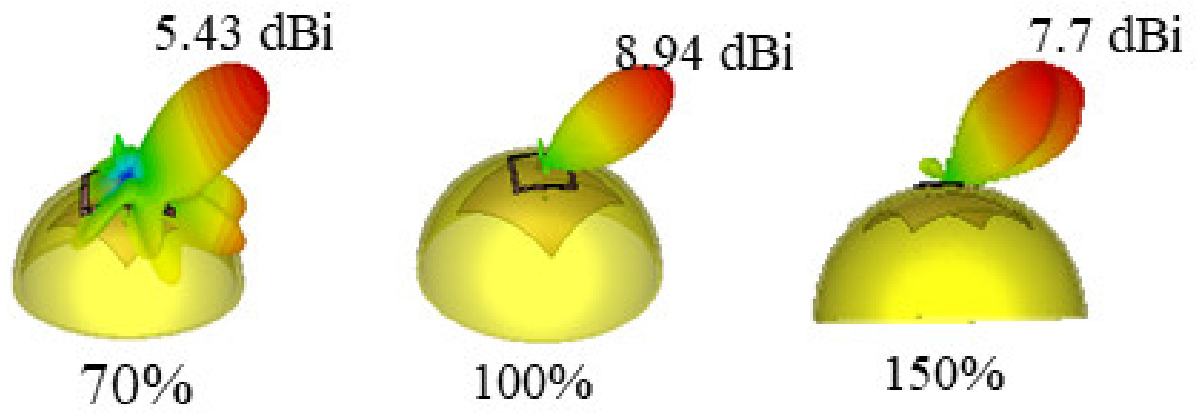

(b) The change in radiation pattern of HSLA when $r$ was varied from $70 \%(192.4 \mathrm{~mm})$ to $150 \%$ (412.3mm).

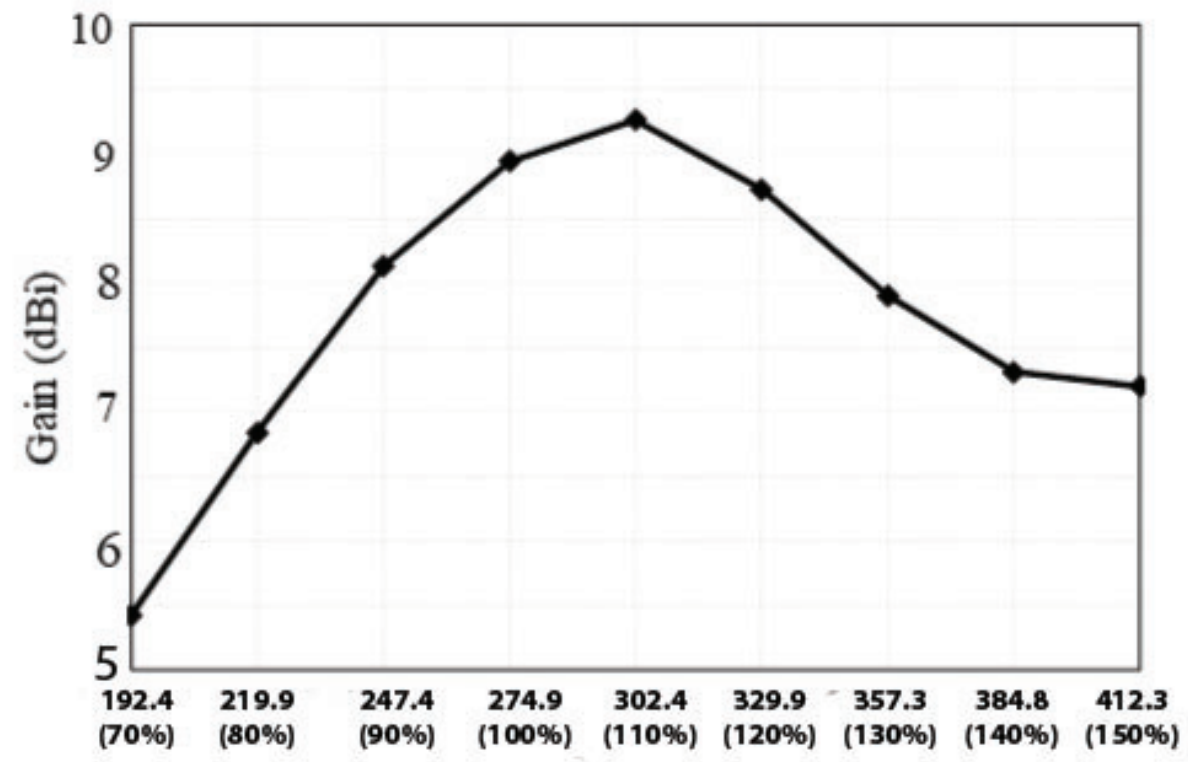

Substrate width $(\mathrm{mm})$

(c) The change in Gain of HSLA when $r$ was varied from $70 \%(192.4 \mathrm{~mm})$ to $150 \%$ (412.3mm).

Fig. 4.9 The change in radiation pattern and Gain of HSLA when curvilinear distance was varied 
thickness was varied from $50 \%(11.25 \mathrm{~mm})$ to $150 \%(33.75 \mathrm{~mm})$ while keeping the $\frac{h_{1}}{h_{2}}$ ratio the same. The change in the HSLA radiation pattern is shown in Fig. 4.8a while the change in Gain of the antenna is shown in Fig. 4.8b. The antenna provides Co-polarization Side Lobe Level (Co-pol SLL) of less than $7 \mathrm{~dB}$ for a substrate thickness between $16.43 \mathrm{~mm}$ $(73 \%)$ and $28.8 \mathrm{~mm}(128 \%)$.

When the radiation patterns were further examined, it was found that the HSLA produced the lowest level of side lobes at the original height of $22.5 \mathrm{~mm}$ (Fig 4.2). The radiation pattern bandwidth of HSLA for $7 \mathrm{~dB}$ SLL criteria was found to be from $2.3 \mathrm{GHz}$ to $2.5 \mathrm{GHz}$.

\subsubsection{Effects of substrate width on radiation pattern}

Furthermore, a study was undertaken to analyse the effects of varying top to rim curvilinear distance, $\boldsymbol{r}$ on the radiation pattern of the antenna. The curvilinear distance $\boldsymbol{r}$ is taken as the curved distance from top of the hemisphere to its rim, as shown in Fig. 4.9a. When the radius of the hemispherical shell, $r_{1}$ is varied the curvilinear distance, $r$ would also vary.

During this study, the antenna's substrate thickness $\left(h_{1}+h_{2}\right)$ was kept constant at 22.5 $\mathrm{mm}$. Curvilinear distance $\boldsymbol{r}$ was varied from $70 \%(192.4 \mathrm{~mm})$ to $150 \%(412.3 \mathrm{~mm})$ referenced to that of the substrate selected for the hemisphere $(r=274.5 \mathrm{~mm})$ accommodating the loop antenna. Tilted radiation patterns with (Co-pol SLL) of less than $7 \mathrm{~dB}$ were also observed for $\boldsymbol{r}$ between $261.2 \mathrm{~mm}-357.34 \mathrm{~mm}$. Outside this range the SLL became more dominant. This is shown in Fig. 4.9.

\subsubsection{Effects of substrate bending on radiation pattern}

The next study covers how the antenna radiation pattern changes with different substrate curvatures while the total surface area of the substrate is kept at same as of HSLA at 1924 $c m^{2}\left(\approx 2 \pi a^{2}\right)$. This in effect is a planar to hemi-prolate transformation. The thickness of the substrate, $h_{1}$ and the thickness of the foam spacer gap, $h_{2}$ were kept the same as before (at $8.5 \mathrm{~mm}$ and $14 \mathrm{~mm}$ respectively). The electrical lengths of the radiating arms of the HSLA were also kept fixed at $96 \mathrm{~mm}$. The HSLA and its ground plane were bent to conform to the surfaces of hemi-prolate shapes.

The $\boldsymbol{c}$ and $\boldsymbol{a}$ values of the 7 stages, of this planar to hemi-prolate transformation, achieved by varying the vertical and horizontal axis ratio, $\frac{\mathbf{c}}{\mathbf{a}}$ of the shell are shown in Table 4.1 along with the observed Gain of the HSLA at each stage.

The Gain of the radiation patterns for the conformal SLA antenna based on hemi-prolate shapes against $\frac{\mathbf{c}}{\mathbf{a}}$ (the vertical to horizontal axis ratio) is plotted in Fig. 4.10b. From Fig. 4.10 it can be observed that the conformal SLA provides SLL free patterns for a range of shell 


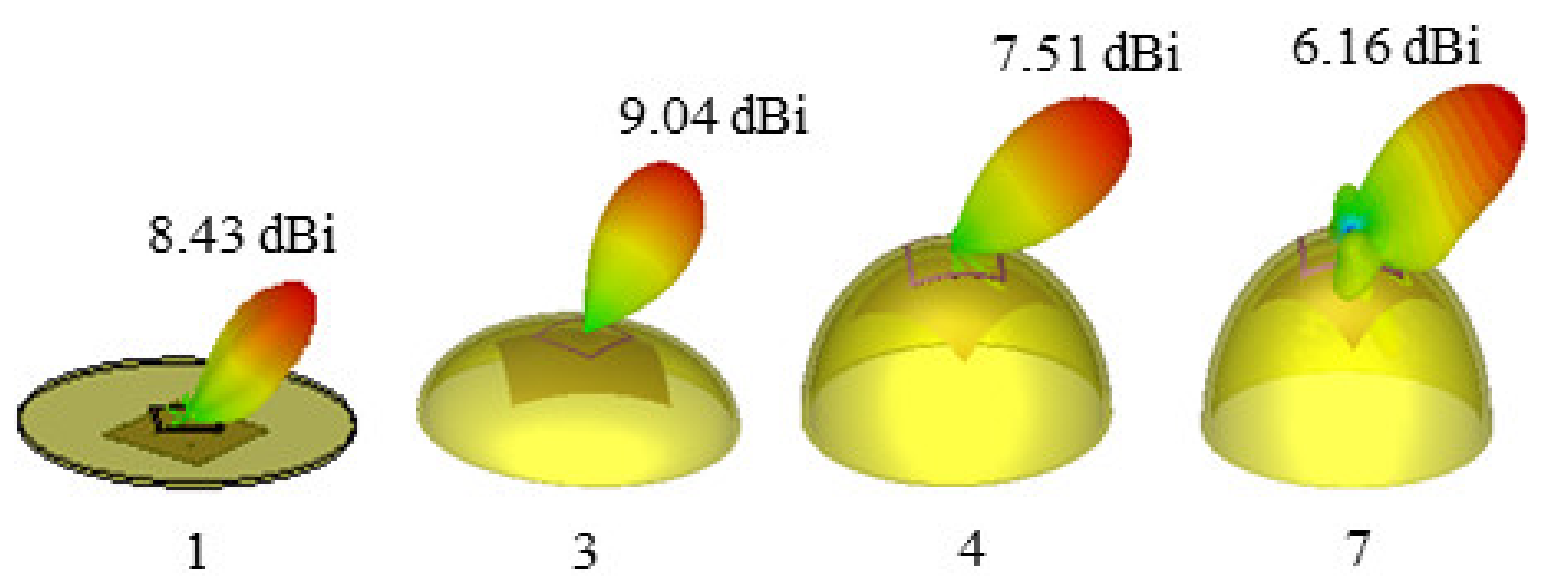

(a) The change in radiation pattern of conformal SLA (marker points 1, 3, 4 and 7) when $\frac{c}{a}$ was varied according to Table 4.1.

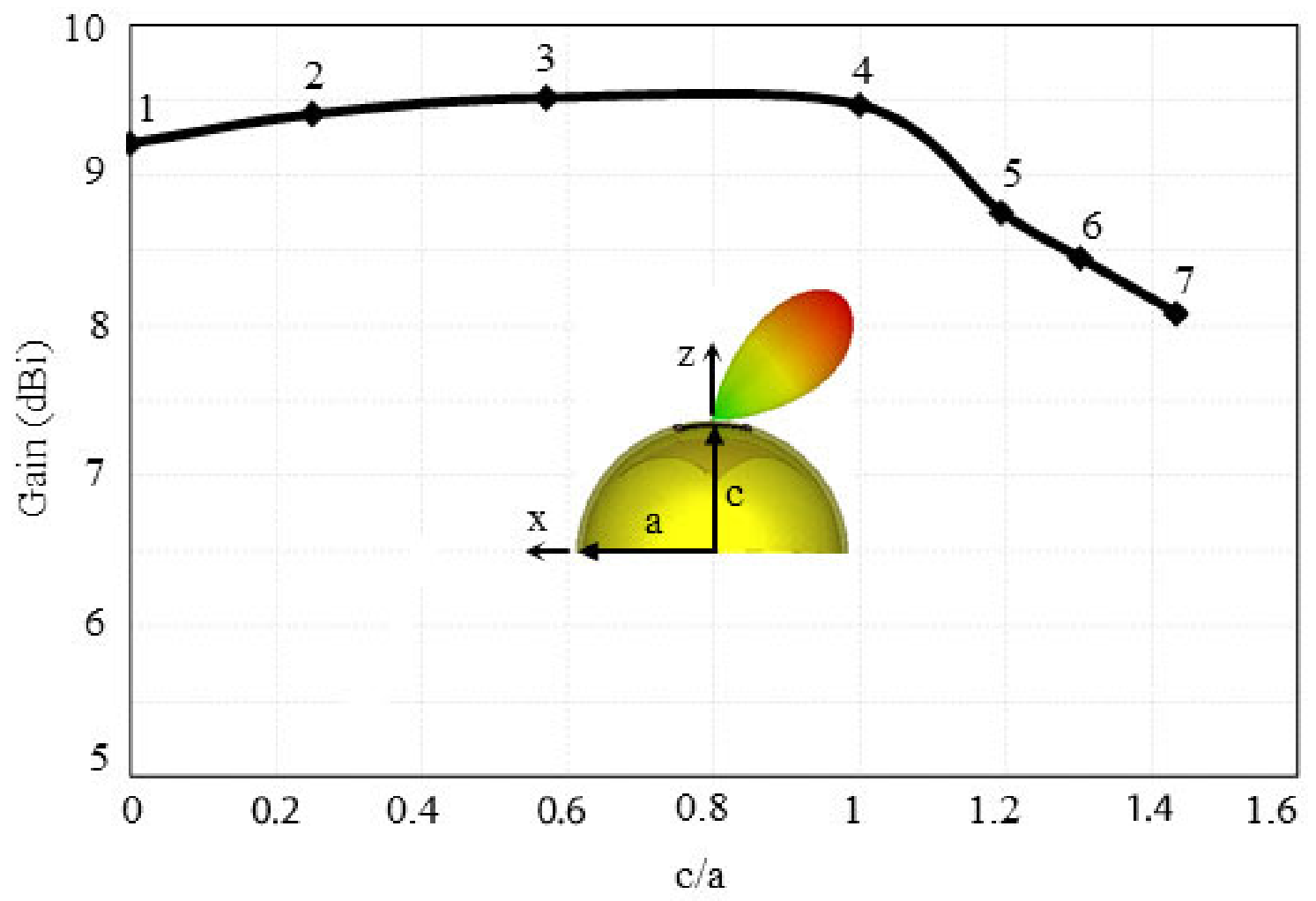

(b) The change in Gain of conformal SLA when $\frac{c}{a}$ was varied according to 4.1.

Fig. 4.10 The effects of substrate bending on conformal SLA 
Conformal 2.4 GHz beam steering antenna for high throughput applications in weak EM environments

\begin{tabular}{|c|c|c|c|c|c|c|c|}
\hline Marker & 1 & 2 & 3 & 4 & 5 & 6 & 7 \\
\hline $\mathrm{c}(\mathrm{mm})$ & 0 & 58 & 117 & 175 & 185 & 190 & 195 \\
\hline $\mathrm{a}(\mathrm{mm})$ & 247 & 232 & 205 & 175 & 155 & 146 & 136 \\
\hline Gain $(\mathrm{dBi})$ & 8.43 & 8.82 & 9.04 & 8.94 & 7.51 & 6.9 & 6.16 \\
\hline
\end{tabular}

Table 4.1 The variation of vertical axis $\boldsymbol{c}$ and horizontal axis $\boldsymbol{a}$ for the 7 shapes of the conformal SLA studied.

substrates with $\mathbf{a}>\mathbf{c}$. In the HSLA case (when $\frac{\mathbf{c}}{\mathbf{a}}=1$ ), the antenna provides amongst the highest reached Gain of $8.94 \mathrm{dBi}$, which is more useful for multipath mitigation and jamming avoidance.

\subsection{Integration of HSLA with $2.4 \mathrm{GHz}$ WiFi system}

\subsubsection{Experimental setup}

The conformal HSLA is integrated with a $2.4 \mathrm{GHz}$ WiFi transmitter system to analyze its beam switching capability in a mobile sensor network environment. In this arrangement the conventional monopole antenna in the WiFi router is replaced with the HSLA. A Hitite ${ }^{\circledR}$ SP4T switch (Devices, 2009) was connected between the 4 ports of the HSLA and the antenna port of the router to switch the beam electronically. A microcontroller based voltage driver circuit operated remotely via Bluetooth controls the RF switch, is shown in Fig 4.11a.

A mobile wireless device (a Windows laptop) is connected to the wireless network of the router. This laptop transceiver antenna for this link is the in-built patch antenna. The laptops using its inbuilt patch antenna reads the Received Signal Strength Indicator (RSSI) of the WiFi signals that are transmitted from the HSLA of the router (Fig. 4.11b). An intelligent beam switching algorithm written in $\mathrm{C \#}$ runs on the laptop (mobile device) which monitors the RSSI (Fig. 4.12). When the signals strength reduces by more than 50\% (i.e. RSSI drops by more than $3 \mathrm{~dB}$ ), it sends the control signals to the microcontroller circuit via Bluetooth to switch the SLA beam in the 4 quadrants in space by switching ON each port sequentially (Fig. 4.1).

During this scanning action, the algorithm reads and records the RSSI from the 4 ports and decides autonomously to switch to the port that provides the highest RSSI. This architecture in effect produced a router, which in real time tracked the mobile device by activating a port that resulted in highest RSSI at the laptop.

As shown in Fig. 4.11b, the mobile node was placed in the 4 quadrants (named A, B, C and D) at an equal distance $(106 \mathrm{~cm})$ away from the HSLA and at an equal height $(77 \mathrm{~cm})$ 


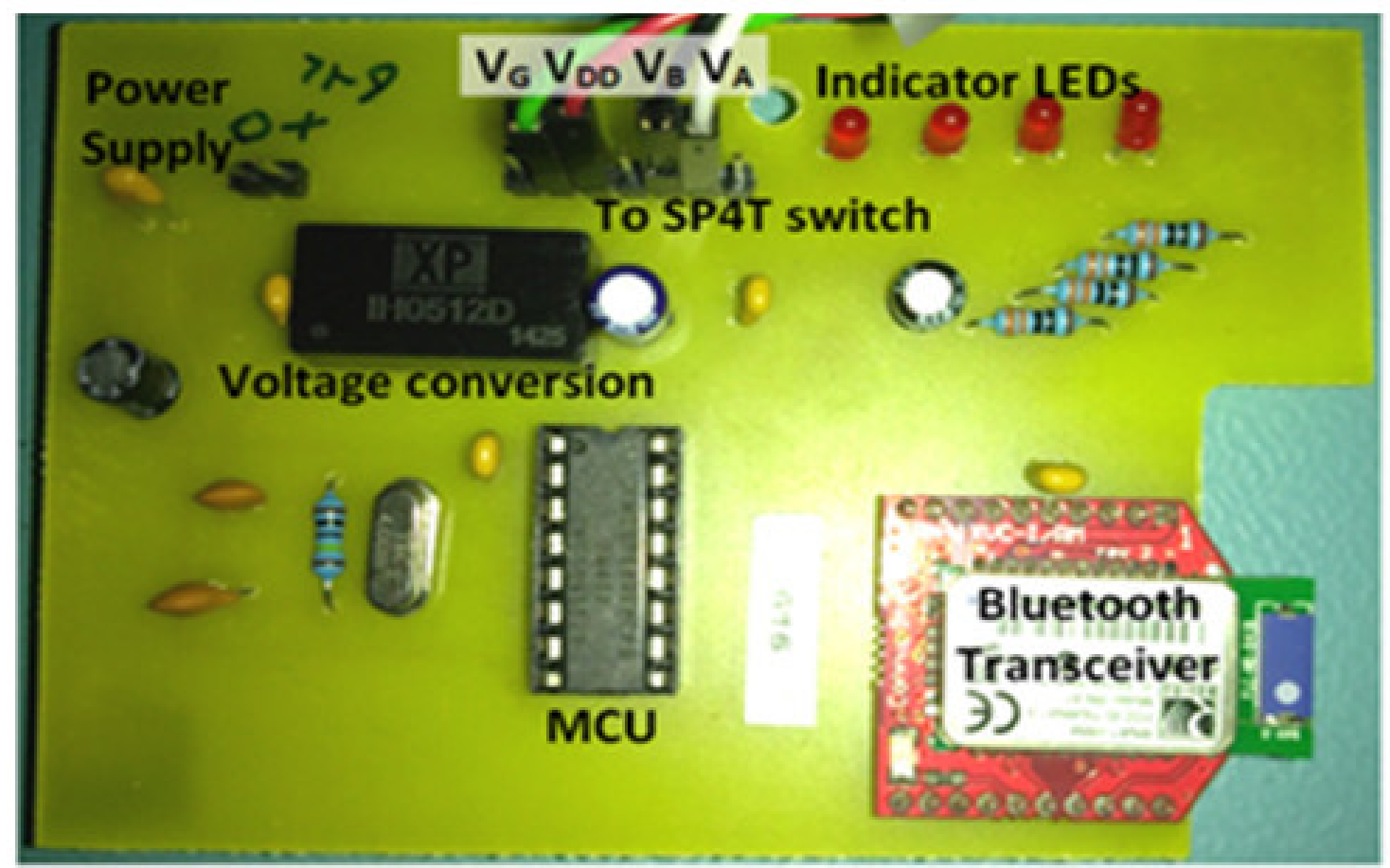

(a) The microcontroller circuit for controlling SP4T electronic switch.

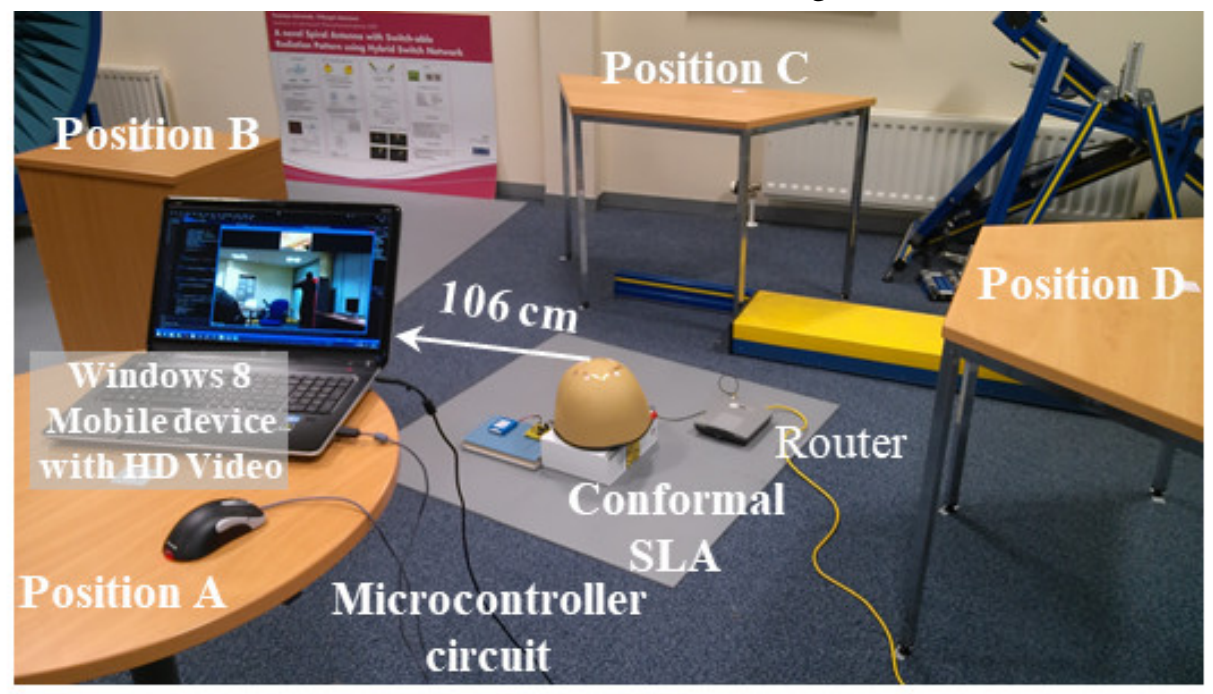

(b) The hardware setup for the experiment.

Fig. 4.11 The microcontroller circuit for controlling SP4T switch and the hardware setup for experiment 


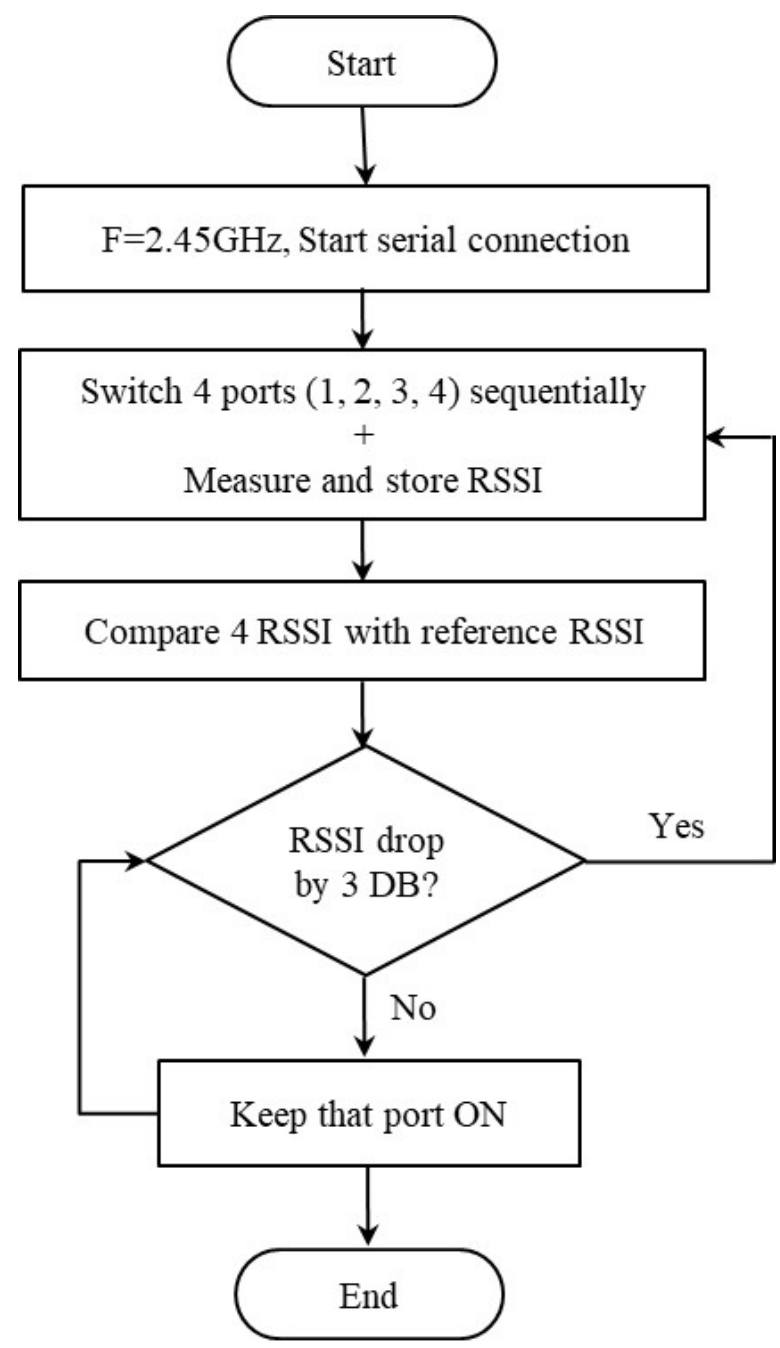

(c)

Fig. 4.12 The automatic beam switching algorithm flowchart 


\begin{tabular}{|l|c|}
\hline Quantity & Loss/Gain \\
\hline Router $T_{x}$ Power & $-27 \mathrm{dBm}$ \\
\hline HSLA antenna Gain & $8.94 \mathrm{dBi}$ \\
\hline$R_{x}$ antenna Gain (estimated) & $3 \mathrm{dBi}$ \\
\hline Loss in SP4T RF Switch & $1.2 \mathrm{~dB}$ \\
\hline Other receiver losses & $12 \mathrm{~dB}$ \\
\hline Expected (LOS) $R_{x}$ signal level & $-69 \mathrm{dBm}$ \\
\hline
\end{tabular}

Table 4.2 Link budget calculation for experimental setup

\begin{tabular}{|c|c|c|c|c|c|c|}
\hline \multirow{2}{*}{ Position } & \multirow{2}{*}{ Port Expected } & \multirow{2}{*}{ Port Selected } & \multicolumn{4}{|c|}{ RSSI at port (dBm) } \\
& & & 1 & 2 & 3 & 4 \\
\hline A & 1 & 1 & -69 & -81 & -81 & -81 \\
\hline B & 4 & 4 & -77 & -78 & -81 & -69 \\
\hline C & 3 & 3 & -76 & -75 & -69 & -77 \\
\hline D & 2 & 2 & -76 & -69 & -73 & -79 \\
\hline
\end{tabular}

Table 4.3 RSSI readings for the four ports at each location during a live HD video call

from ground. The HSLA (i.e. base station antenna) was placed at the centre while the mobile device was being moved from one quadrant to another with its beam switching code running. Due to the symmetric nature of the conformal HSLA, it was expected that at each of the 4 ports of the HSLA, it would produce an identical beam, when switched ON. Hence, this would result in the mobile device receiving the same RSSI at each location (A, B, C and D) if the correct port for that quadrant is switched ON using the algorithm.

Table 4.2 shows the link budget calculation for the $2.4 \mathrm{GHz}$ WiFi transmitter system used for the experiment. On purpose transmit power is kept at $-27 \mathrm{dBm}$, much lower than standard WIFI transmit power. This is done so as to create a weak electromagnetic environment within a small laboratory area. The beam switching algorithm under testing performed accurately as expected, selecting the port with the highest RSSI at each of the 4 locations.

In all 4 positions, the best port provided an RSSI of $-69 \mathrm{dBm}$ which demonstrated that the ports are identical and HSLA is symmetric. This is shown in Table 4.3, which shows autonomous appropriate port activation in HSLA based on the location of the mobile laptop.

The effects of beam switching using the automatic beam switching algorithm to track the user during a live HD video conferencing call is shown in Fig. 4.13. When the beam switching algorithm was switched off, as a controlled experiment, the latency of the received video stream was $92 \mathrm{~ms}$ and there was a packet loss of $85.5 \%$ and $66.7 \%$ from the sent and received video streams respectively. The maximum receive resolution that could be supported 


\begin{tabular}{|c|c|c|}
\hline \multicolumn{3}{|c|}{ Zoom Meeting ID: 185-961-757 Participant ID: 27} \\
\hline \multicolumn{2}{|c|}{ Settings } & \multirow[b]{2}{*}{ Screen Sharing } \\
\hline Overall & Audio ! & \\
\hline Item name & Send & Receive \\
\hline Latency & $101 \mathrm{~ms}$ & $92 \mathrm{~ms}$ \\
\hline Jitter & $4 \mathrm{~ms}$ & $4 \mathrm{~ms}$ \\
\hline Packet loss - Avg(Max) & $85.5 \%(96.3 \%)$ & $66.7 \%(98.0 \%)$ \\
\hline Resolution & $640 \times 360$ & $640 \times 360$ \\
\hline Frame Per Second & $24 \mathrm{fps}$ & $21 \mathrm{fps}$ \\
\hline
\end{tabular}

Poor Internet connection maybe affecting your meeting experience; please close other applications or check your network bandwidth.

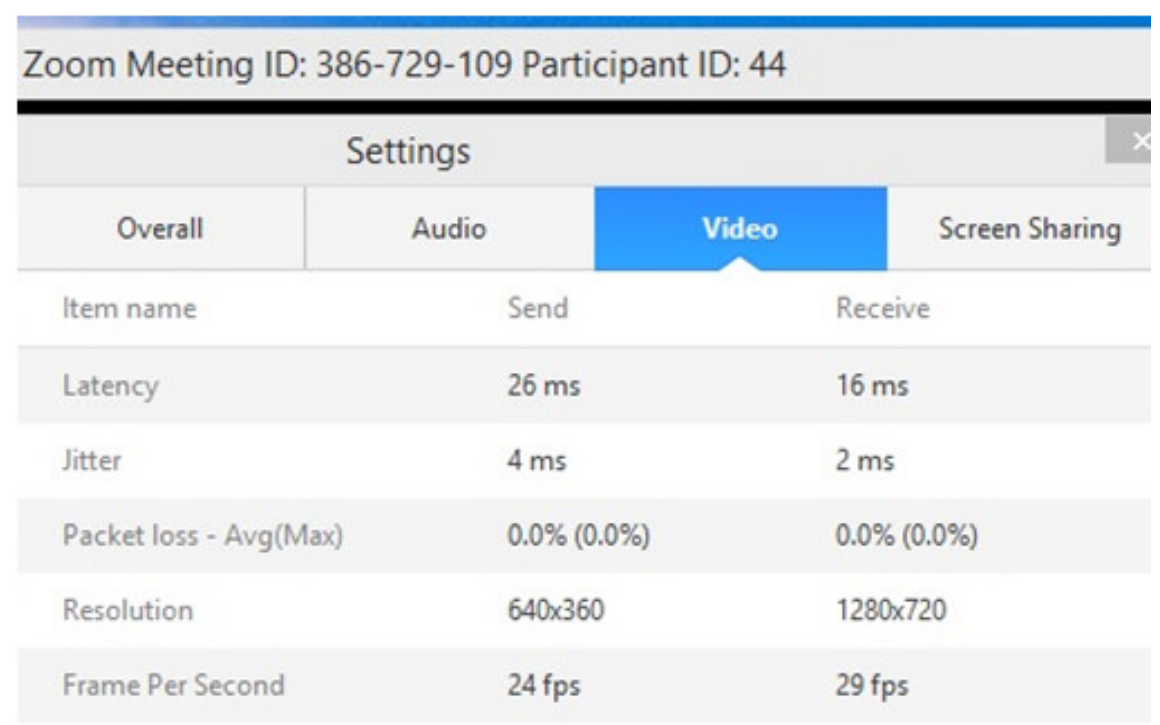

Fig. 4.13 Screenshots of video call statistics when (Top) automatic beam switching was not used, (Bottom) when automatic beam switching was used. 
was 640 pixels $\times 360$ pixels with a frame rate of $21 \mathrm{fps}$. There were visible distortion that appeared in the video stream when the mobile device was moved around.

When the automatic beam switching algorithm was switched $\mathrm{ON}$, the video statistics improved drastically. The latency decreased to $16 \mathrm{~ms}$ from $92 \mathrm{~ms}$ earlier while the packet loss was zero for both the sent and received video streams.

Under automatic beam switching, it ensured an uninterrupted video call, while the mobile device was being moved around from positions A to B to $\mathrm{C}$ to $\mathrm{D}$. The video call reception was able to sustain a resolution of 1280 pixels $\times 720$ pixels (compressed in transmission). In terms of throughput it was found that when beam steering algorithm was ON, it supported error free data rate of $7.5 \mathrm{Mbps}$. When the algorithm was OFF, the data rate went down to near 0.1 Mbps.

\subsection{Conclusion}

A novel switch beam conformal SLA for sensor networks is introduced for sustained high throughput applications. The SLA printed on a hemispherical shell (referred to as HSLA) gives a beam of $46^{\circ}$ tilt and of $8.94 \mathrm{dBi}$ directivity. The measured antenna beam and the return loss are in good agreement with the calculated model. The HSLA was integrated with a full $2.4 \mathrm{GHz}$ WiFi router transceiver system and was tested using a mobile laptop roaming device.

This device had an automatic beam switching algorithm that controlled and switched the router's radiated beam. The algorithm provided a seamless beam steering as the mobile device was moved around the router. It was found that beam steering enabled a sustainment of high throughput for a live HD video call. In a weak electromagnetic environment, it was found that beam steering enabled a throughput of 7.5 Mbps and when it was switched OFF the throughput dropped to $0.1 \mathrm{Mbps}$. 



\section{Chapter 5}

\section{HSLA Integrated with Raspberry Pi for a mobile user using high throughput applications}

\subsection{Introduction}

In this chapter, I present a complete system consisting of the beam steerable Hemispherical Square Loop Antenna (HSLA) presented in chapter 4, integrated with a Raspberry Pi2. The aim of this experimental setup is to optimize the spectrum efficiency in a rough EM environment.

It is demonstrated that in a weak EM environment (i.e received signal close to the noise floor), the HSLA enables a throughput increment by a factor over 7 (0 to $7 \mathrm{Mbps}$ ). In quality terms this means sustaining full HD communications. Effectively, it improves the system spectrum efficiency for the $2.4 \mathrm{GHz}(802.11 \mathrm{~b} / \mathrm{g} / \mathrm{n}) \mathrm{WiFi}$ band.

Automatic pattern adaptation is achieved using a smart 'EM sense, scan, analyse and lock' algorithm running on Raspberry Pi which uses a hybrid SC and SEC scheme described in Chapter 3. The Pi tracks the direction of signal arrival for the highest RSSI (Received Signal Strength Indicator). The Pi's algorithm controls the radiation from the HSLA in one of the four possible tilted beam directions by utilizing a RF SP4T switch.

The uniqueness about the system is that it only uses single antenna for both sensing and communication. The algorithm works at the application layer of the OSI 7 layer model (IEEE, 1999), that controls the RF switch and antenna patterns at the physical layer. Thus, the entire middle protocol layers are untouched. This system can easily be retrofitted to existing non-adaptive communication systems. 


\section{HSLA Integrated with Raspberry Pi for a mobile user using high throughput applications}

\subsection{Background}

With emerging Fifth generation $(5 \mathrm{G})$ mobile communication systems the growing demand for high-throughput wireless communications with ubiquitous coverage is enabling arrival of the new antenna technologies. A special aspect of future high speed mobile internet access will be to enable high quality video transmissions. Over the next three years $2 / 3^{\text {rd }}$ of the wireless traffic will be video (Aloman et al., 2015; VNI, 2017), such as High Definition (HD) video conferencing (Yaacoub et al., 2012), video on demand, MobiTv (TV, 2016), electronic classroom (Yaacoub et al., 2012) and WiFi telecast in smart homes (Home, 2016). With the new extended bandwidth allocation in 5G, a multi-gigabit / sec data speed will be realizable (Coomans et al., 2015; Wang et al., 2014).

However, those speeds will only work in areas with a high SIR (Signal to Interference Ratio). In situations where a device is operating in a weak EM environment, (e.g. away from the base stations, in a multipath rich environment or in a high interference zone) the data rates will drop sharply, in line with the Shannon capacity (Shannon, 1949). These low SIR operating conditions will degrade the user experience and expectations. One clever way to solve this degradation is to use high Gain beam-steering antennas. The high Gain will increase a wireless device received power. Beam-steering would enable the device to navigate away from interferences and noise. Both these factores together will push up the effective SIR and in turn, the spectrum capacity (Choudhury et al., 2006; Liu et al., 2009; Navda et al., 2007).

Using the system that I developed, I expect to demonstrate using a real world example with throughput numbers, how in a weak EM environment, a beam-steering antenna can make a significant difference. This improvement is in fact, by over a factor of 100. I achieve this by designing and practically implementing a low cost high Gain intelligent beam steering antenna system.

I have used off-the-shelf components to ensure compatibility with future consumer wireless systems. I chose $2.45 \mathrm{GHz}$ as the test frequency and OFDM WiFi as the test protocol. This choice was underpinned by the fact that WiFi is a mature technology widely used in consumer products worldwide, making it easier to test and integrate this product with many plug-and-play consumer wireless devices.

For the beam-steering antenna I selected single element four feed based Square Loop Antenna (SLA). It is similar to the antennas mentioned in Deo et al. (2010); Mehta and Mirshekar-Syahkal (2007); Mehta et al. (2006); Pal et al. (2014c, 2008, 2009), but is the first SLA developed conformal to a hemispherical platform. An important aspect of $5 \mathrm{G}$ would be high speed connected cars. I selected full hemispherical conformal shape to ensure that our system would easily adapt to various vehicular top and side platforms. In addition, 
the conformal shape is relevant for implementation in the future technologies (e.g. Internet of Things) in which wireless sensors platforms would be of random shapes. The antenna radiates four $9.2 \mathrm{dBi}$ Gain tilted beams in a $90^{\circ}$ azimuth space (single quadrant), one beam corresponding to each of the feeds.

Using a Single Pole Four Throw (SP4T) RF switch (Devices, 2009) the antenna is capable of switching feeds sequentially one at a time and it can steer a high Gain beam in the full $360^{\circ}$ azimuth space. Whilst I could have used phased arrays for beam-steering, that choice would have been expensive, lossy and importantly it would have required large space area for incorporating multiple antenna elements (Bai et al., 2011; Daly and Bernhard, 2010; Pal et al., 2015; Wu et al., 2010a,b).

The intelligent four beam steering was done with the help of Raspberry Pi2 model B (Foundation, 2015). The Pi provides control signals for controlling the RF switch. A smart 'Electromagnetic sense, Analyze and Lock' algorithm was developed using $\mathrm{C}$ language which sits on the Pi. The algorithm enables antenna to ensure that the communication link is always locked in the strongest SIR / RSSI direction.

Using a full High Definition (HD) video conference network, I aim to demonstrate the effectiveness of the pattern adaptive system in sustaining the uninterrupted HD quality video. Hence, I demonstrate that in that weak signal environment a high Gain beam-steering was the difference between full HD communication and no communication at all.

\subsection{Hemispherical Square Loop Antenna (HSLA)}

Figures 5.1 and 5.2 show the transition of Planar SLA (PSLA) to Hemispherical SLA (HSLA). Fig. 5.1 shows the top and side view of the PSLA on a circular substrate. The square loop is placed on top of a circular substrate having a radius of $247 \mathrm{~mm}$ (horizontal radius, $r_{h}$ ). The antenna substrate consists of a stack of two dielectric layers. The dielectric material Kevlar $\left(\varepsilon_{r}=3.4\right)$ is used as top layer which has a height of $h_{1}=8.45 \mathrm{~mm}$. The bottom layer has a height $h_{2}=14 \mathrm{~mm}$ and is made of Rohacell 51 foam $\left(\varepsilon_{r}=1.08\right)$. Hence, the total height of the antenna is $22.45 \mathrm{~mm}$. The radiating square loop is composed of four copper strips having a length $\boldsymbol{l}=96 \mathrm{~mm}$ and a track width $\boldsymbol{w}=5 \mathrm{~mm}$. The loop is excited at four middle points $(\mathbf{A}, \mathbf{B}, \mathbf{C}$ and $\mathbf{D})$ of the four arms by four vertical probes having a diameter of $1.3 \mathrm{~mm}$. The probes are connected to the four standard SMA (SubMiniature version A) ports $A_{0}, B_{0}, C_{0}$ and $D_{0}$, respectively at the bottom of ground plane. The square shaped metallic (Copper) ground plane has an area of $175 \mathrm{~mm} \times 175 \mathrm{~mm}$ and it is rotated by $45^{\circ}$ in the XY-plane with respect to the square loop. 


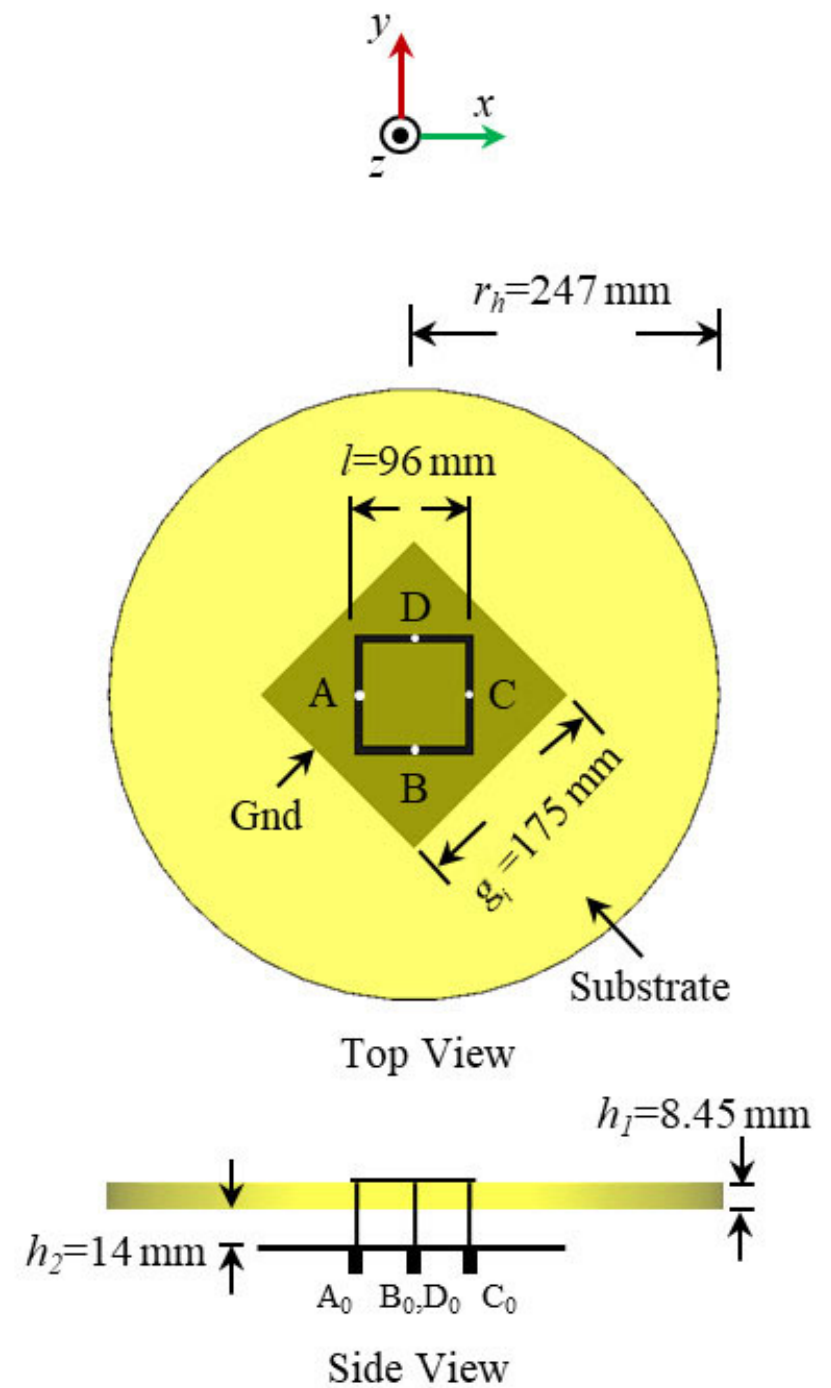

Fig. 5.1 Top view and side view of the planar SLA on a circular substrate. 

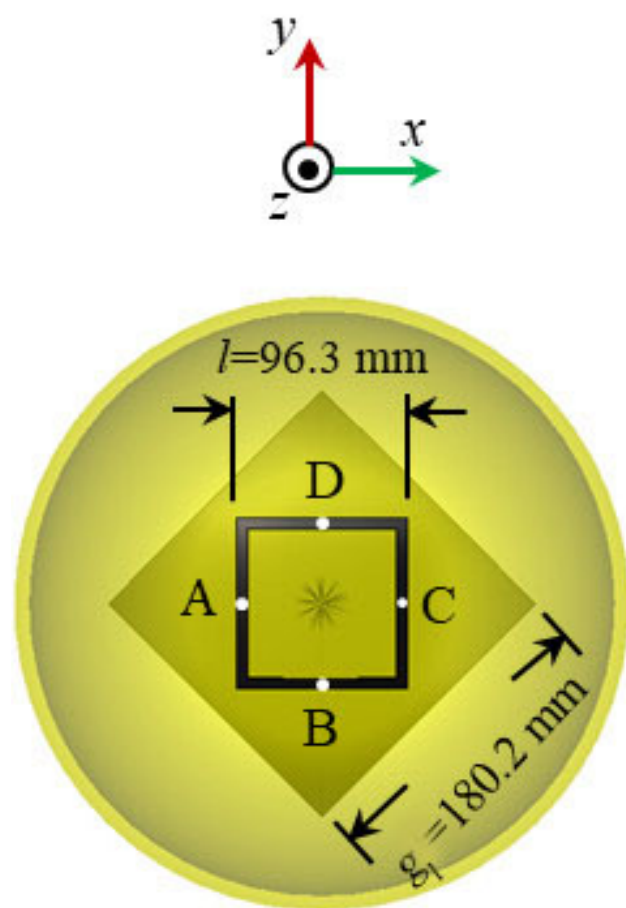

\section{Top View}

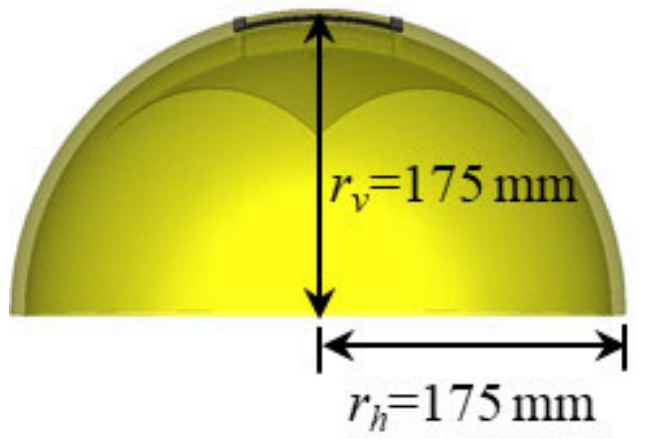

Side View

Fig. 5.2 Top view and side view of SLA on a hemispherical substrate. 
Fig. 5.2 shows the PSLA conformed to HSLA. This was achieved by bending the PSLA from its sides in which the middle portion of the ground plane along with substrate, feeds and square loop are raised. In order to keep the surface area constant, the radius was reduced to a horizontal radius of $r_{h}=175 \mathrm{~mm}$, and a vertical radius of $r_{v}=175 \mathrm{~mm}$. The ground plane was stretched to $180.2 \mathrm{~mm} \times 180.2 \mathrm{~mm}$. The length of each strip of square loop only increased by $0.3 \mathrm{~mm}$ in the PSLA to HSLA transition. 

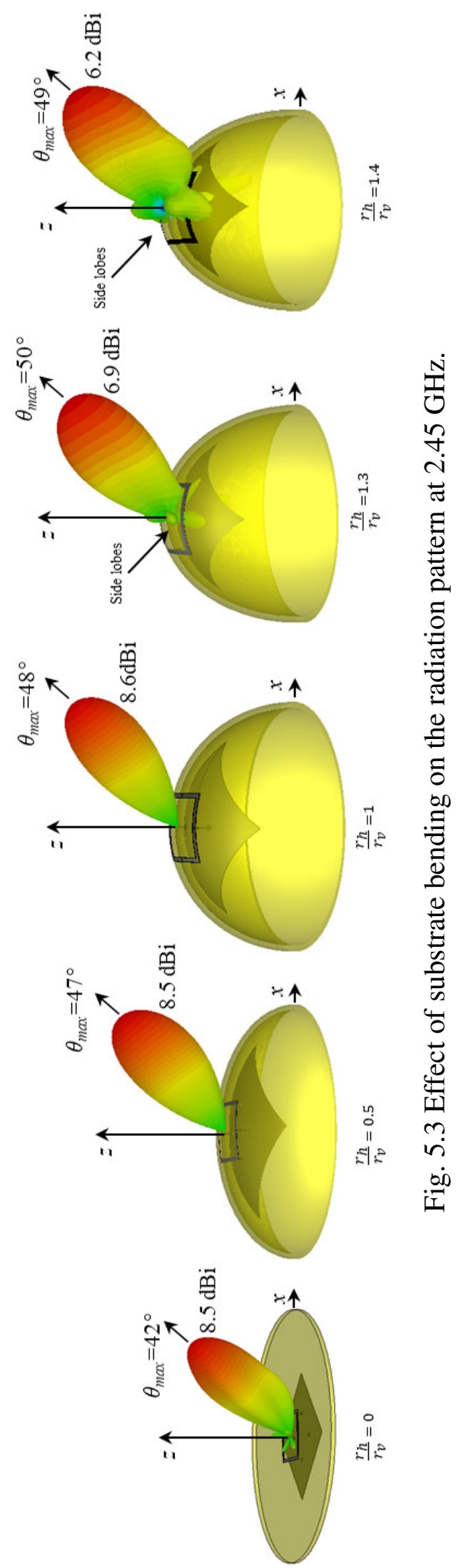

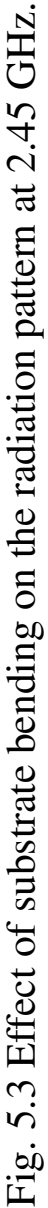




\section{HSLA Integrated with Raspberry Pi for a mobile user using high throughput applications}

Fig. 5.3 shows the effect of the substrate bending on the antenna radiation patterns at $2.45 \mathrm{GHz}$ when the ratio of $r_{h}$ to $r_{v}$ is varied. When only port $A_{0}$ is excited and the remaining ports $\left(B_{0}, C_{0}\right.$ and $\left.D_{0}\right)$ are open-circuited the antenna provides a linearly polarized tilted beam $\left(\theta_{\max }=48^{\circ}\right)$ directed away from the excited port $A_{0}$ (i.e. $\phi_{\max }=0^{\circ}$ ). It is observed that when $\frac{r_{h}}{r_{v}}$ is varied from 0 to 1 (Fig. 5.3), beam tilt angle varies from $\theta=42^{\circ}$ to $\theta=48^{\circ}$ and that the Gain stays nearly constant. It was observed that if the substrate is bent beyond $\frac{r_{h}}{r_{v}}>1.3$, magnitude of side lobe increases and pattern becomes distorted. The hemispherical configuration $\left(\frac{r_{h}}{r_{v}}=1\right)$ of the antenna has the highest tilt angle and Gain.
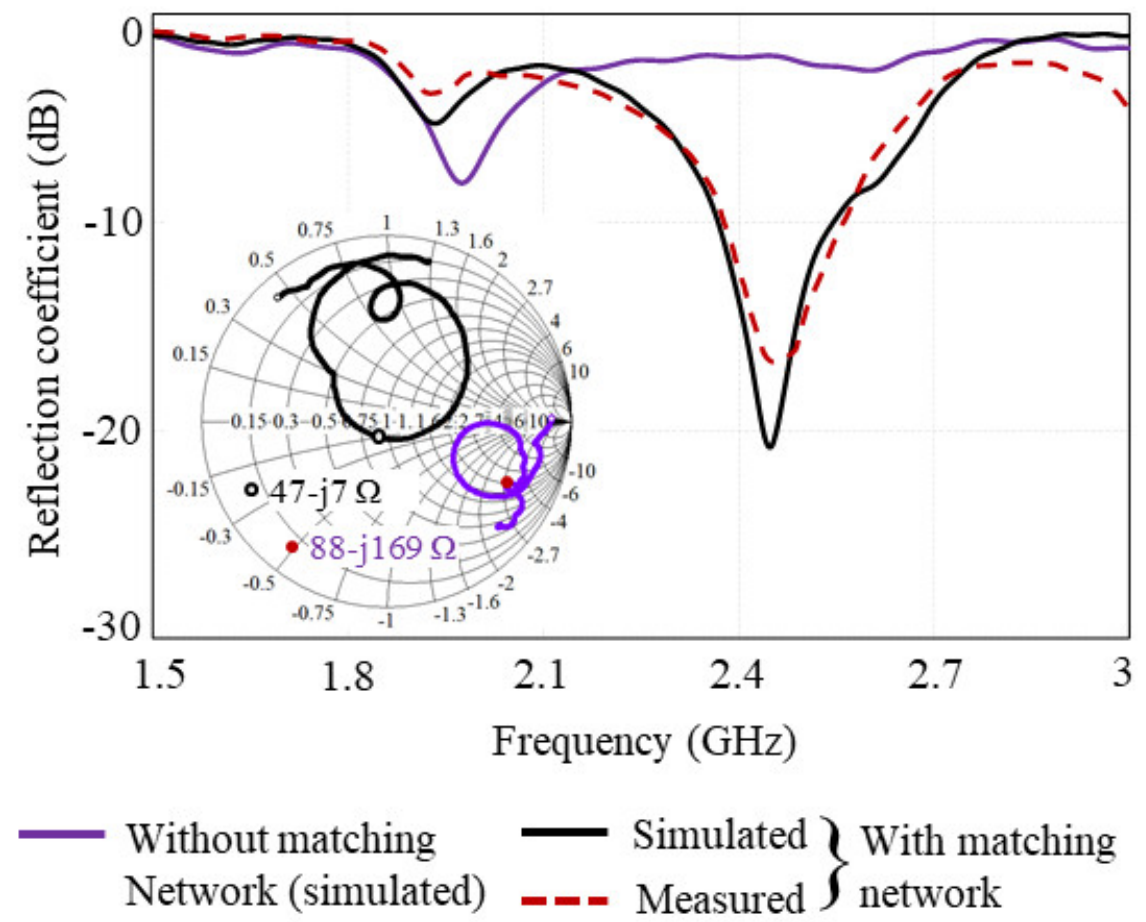

Fig. 5.4 Reflection coefficient and (Inset) the input impedance of the HSLA.

Fig. 5.4 shows the frequency response of the reflection coefficient $\left(\left|S_{11}\right|\right)$ and the input impedance of the HSLA. The antenna shows an input impedance of $88-j 169 \Omega$ at the $2.45 \mathrm{GHz}$. This causes an impedance mismatch with the standard $50 \Omega$ excitation source. Consequently, the antenna exhibits a poor reflection coefficient $\left(\left|S_{11}\right| \approx-3 \mathrm{~dB}\right)$ at the operating frequency. A two-section stepped coaxial transmission line matching circuits are employed to match the antenna to the switch.

Figures 5.5a and 5.5b show the simulated model and fabricated prototype of two-section stepped transmission line matching network. The matching network on the microstrip has two $9 \mathrm{~mm}$ long SMA standard connectors on its either sides. The matching network transforms 


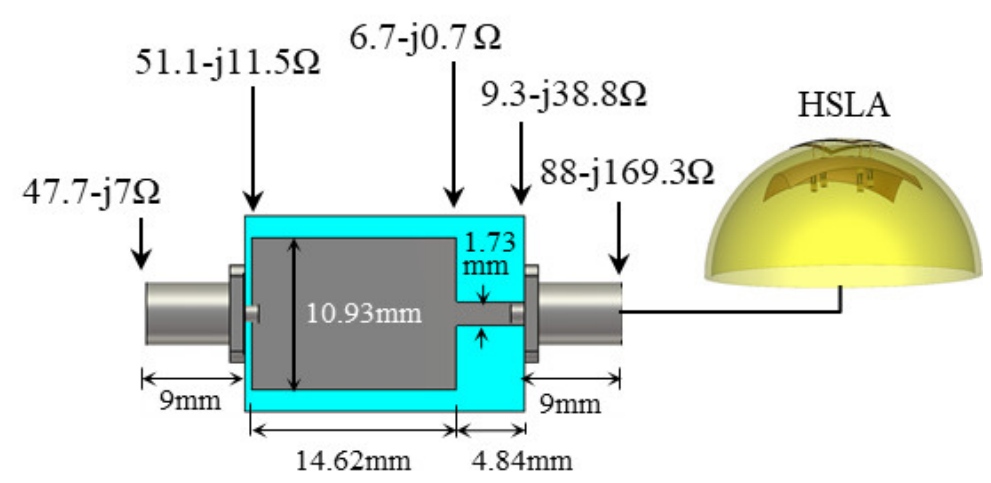

(a) Simulted model

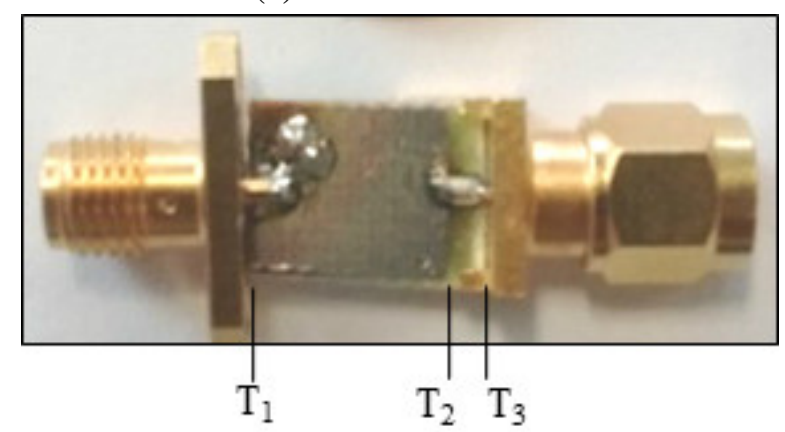

(b) Fabricated prototype

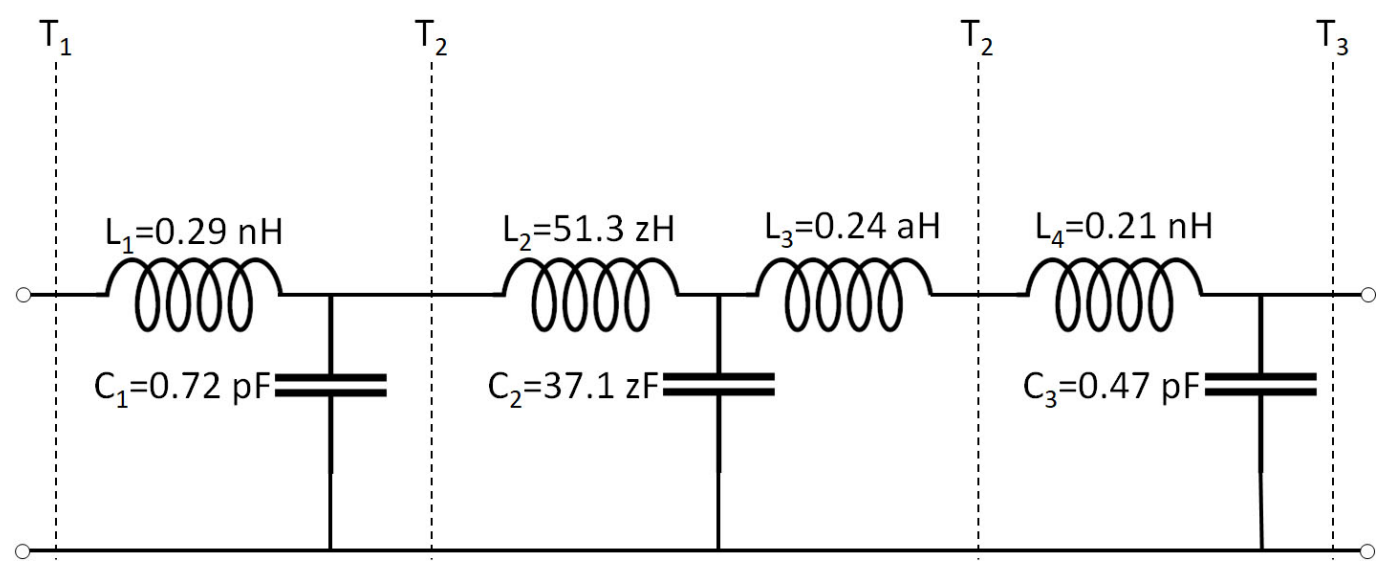

(c) Equivalent circuit

Fig. 5.5 The Microstripline component for matching the input impedance. 


\section{HSLA Integrated with Raspberry Pi for a mobile user using high throughput applications}

$88-j 169.3 \Omega$ from the input of HSLA to $50 \Omega$. The initial values of characteristic impedance and length of the microstrip line were obtained from the work presented by Rosloniec (1994) and subsequently, an optimizer of CST Microwave Studio (CST-MWS) (CST, 2013) was used for achieving the final values, shown in Fig. 5.5.

The matching network was developed using an FR4 substrate having permittivity of 4.8 and a height of $1.6 \mathrm{~mm}$. Fig. 5.5c shows the equivalent circuit of the matching network, which were determined from the network parameter extraction method of CST-MWS. Fig. 5.4 shows the reflection coefficient and input impedance of the HSLA when integrated with matching networks. With the matching network the HSLA operates efficiently $\left(\left|S_{11}\right|<-10\right.$ $\mathrm{dB})$ over $2.4 \mathrm{GHz}(802.11 \mathrm{~b} / \mathrm{g} / \mathrm{n})$ WiFi frequency band with an impedance bandwidth of 180 $\mathrm{MHz}(2.36 \mathrm{GHz}$ to $2.54 \mathrm{GHz})$.

When port $A_{0}$ is excited and remaining ports $\left(B_{0}, C_{0}\right.$ and $\left.D_{0}\right)$ are open circuited, the HSLA provides a linearly polarized beam, with a tilt of $\theta_{\max }=48^{\circ}$ from the zenith (Z-axis). The beam is pointed away from the excited port, i.e. in the direction of $\phi_{\max }=0^{\circ}$. With the matching network the HSLA provides a Gain of $9.2 \mathrm{dBi}$ in the direction of maximum radiation with a radiation efficiency of $87 \%$ over the impedance bandwidth. The main radiation beam is linearly polarized in the direction of maximum radiation. The magnitude of the cross-polarized $\left(E_{\Phi}\right)$ component is well below from that of the co-polarized component $\left(E_{\theta}\right)$, i.e. $\left|E_{\theta}\right|>\left|E_{\Phi}\right|$ by over $40 \mathrm{~dB}$.

It is shown in Fig. 5.6 that the SLA maintains its pattern shape over the radiation pattern bandwidth of $250 \mathrm{MHz}$ ( 2.35 to $2.6 \mathrm{GHz})$. A variation of $10^{\circ}\left(46^{\circ}\right.$ to $\left.56^{\circ}\right)$ in beam tilt angle and a variation $0.7 \mathrm{~dB}(8.5 \mathrm{dBi}$ to $9.2 \mathrm{dBi})$ in Gain is observed over the radiation pattern bandwidth. Outside the pattern bandwidth, the radiation patterns are distorted due to split beam.

Since, the square loop is symmetrical with respect to the centre point of the whole structure, the radiation patterns of other feeding ports $\left(B_{0}, C_{0}\right.$ and $\left.D_{0}\right)$ are similar to that of port $A_{0}$. Therefore, when any of the four feeding ports are excited one at a time, while remaining ports are open circuited, the HSLA provides a tilted beam of $\theta_{\max }=48^{\circ}$ in four different space quadrants of $\phi_{\max }=0^{\circ}, \phi_{\max }=90^{\circ}, \phi_{\max }=180^{\circ}$ and $\phi_{\max }=270^{\circ}$. Thus, by switching the RF input among the feeding ports the HSLA manoeuvres its radiation beam over the four quadrants to scan the entire $360^{\circ}$ space in front of the antenna. I achieved this beam steering by using an RF switch and an intelligent algorithm for spectrum capacity enhancements. It is described in the next section. 

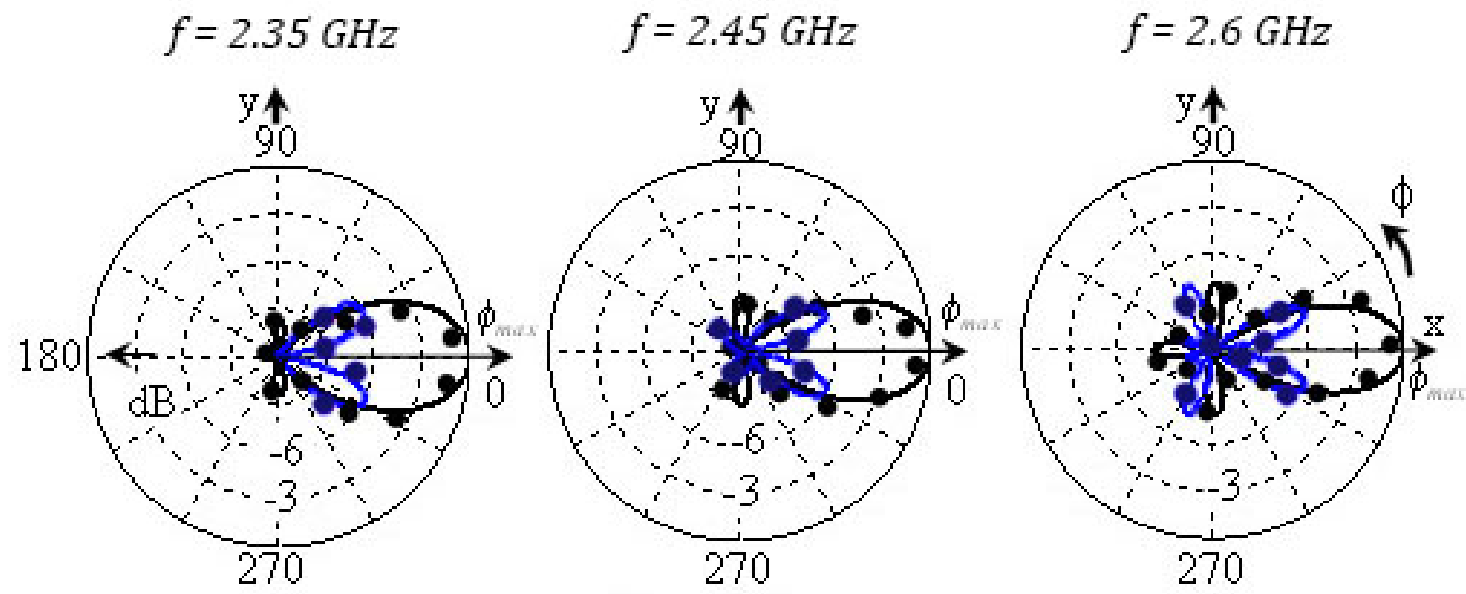

Azimuth plane

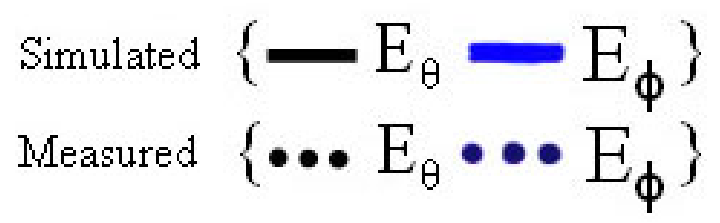

(a) The Polar Plots in the Azimuth Plane.

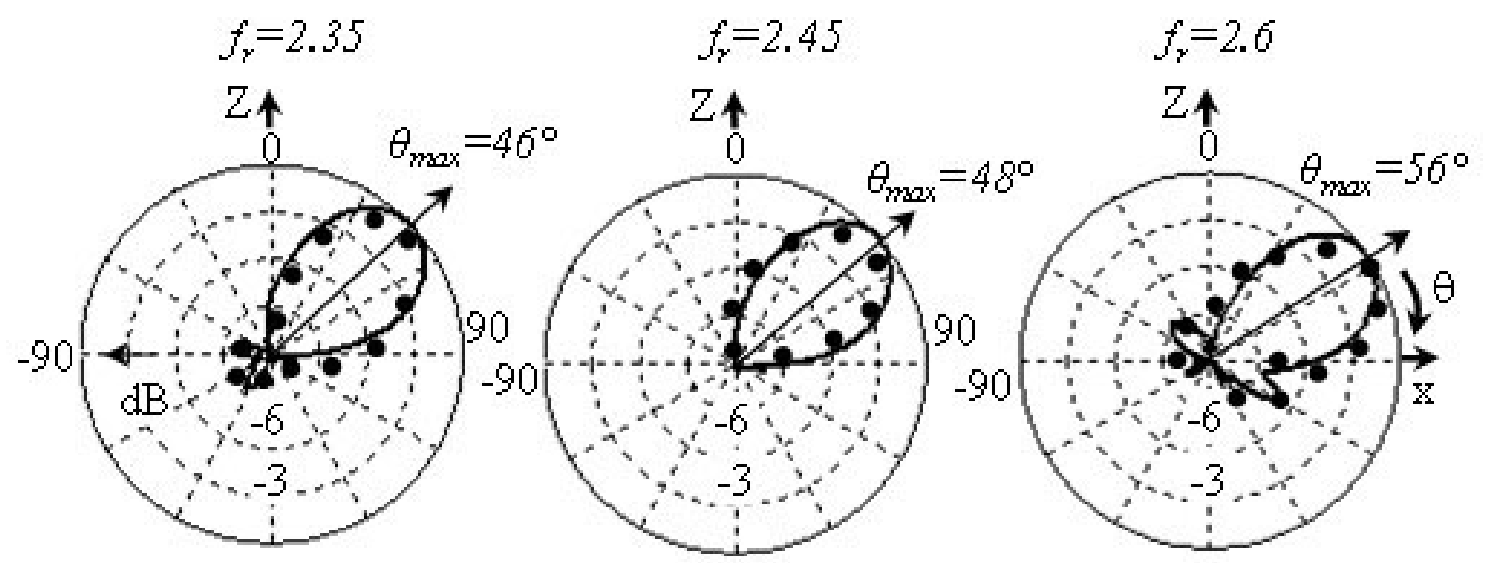

Elevation plane

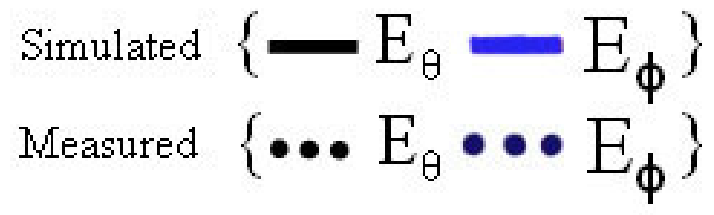

(b) The Polar Plots in the Elevation Plane.

Fig. 5.6 The Polar Plots of the HSLA in the 1Azimuth plane and the Elevation plane for 2.35 $\mathrm{GHz}, 2.45 \mathrm{GHz}$ and $2.6 \mathrm{GHz}$. 


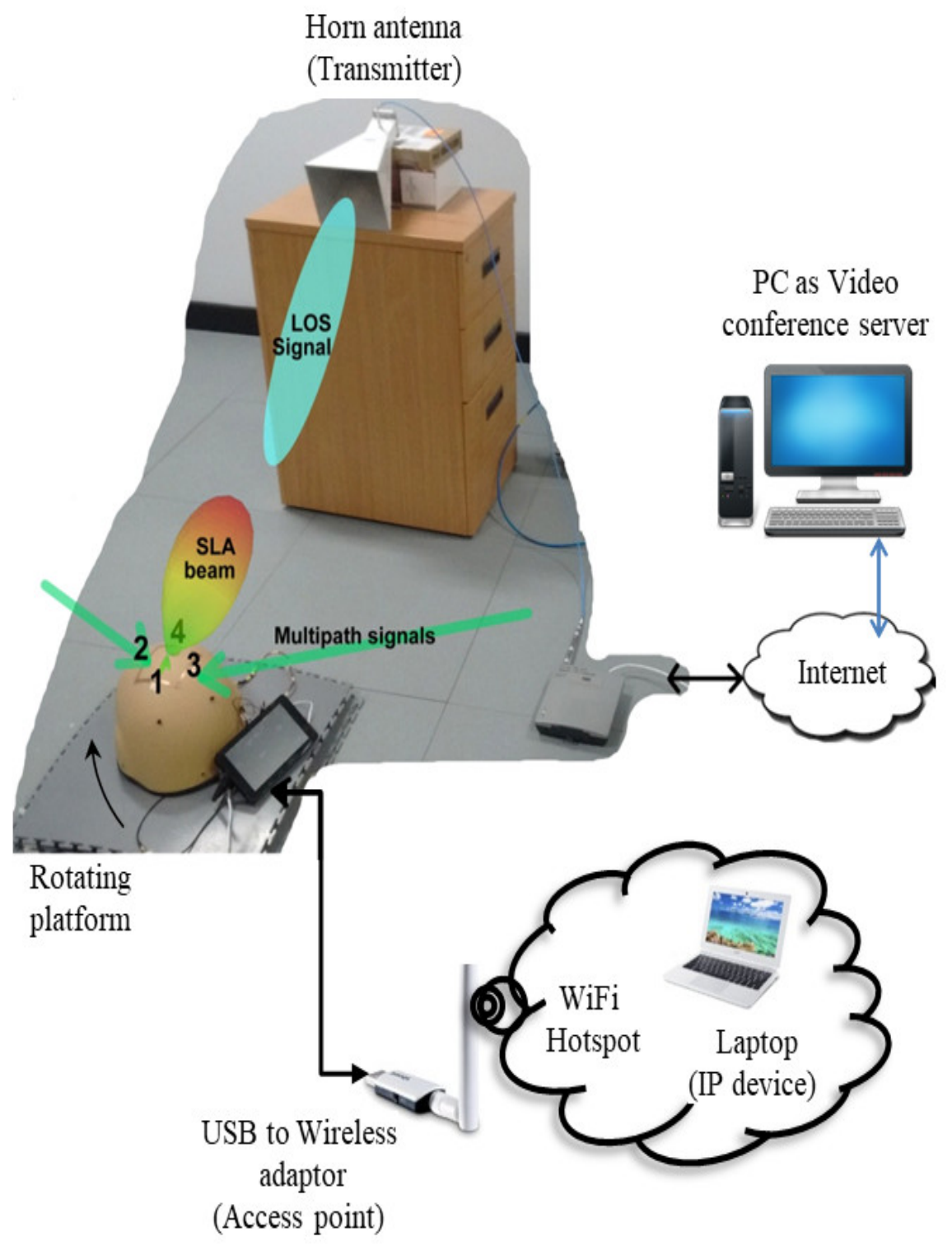

Fig. 5.7 Schematic of the beam-steering system. 


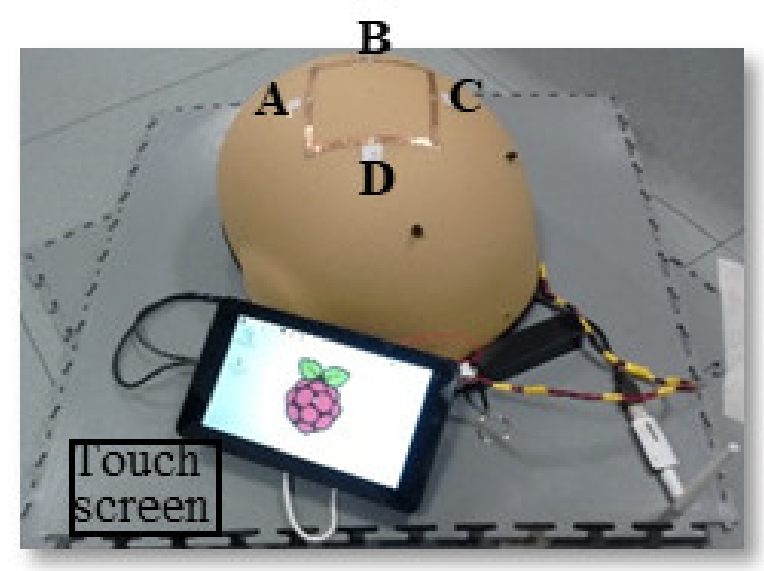

HSLA and Raspberry Pi
Switch control

voltages

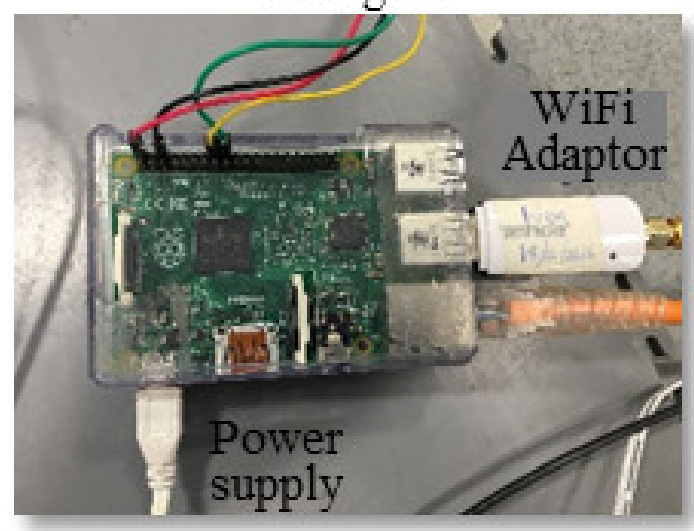

Raspberry Pi

(a) The HSLA connected to Raspberry Pi.

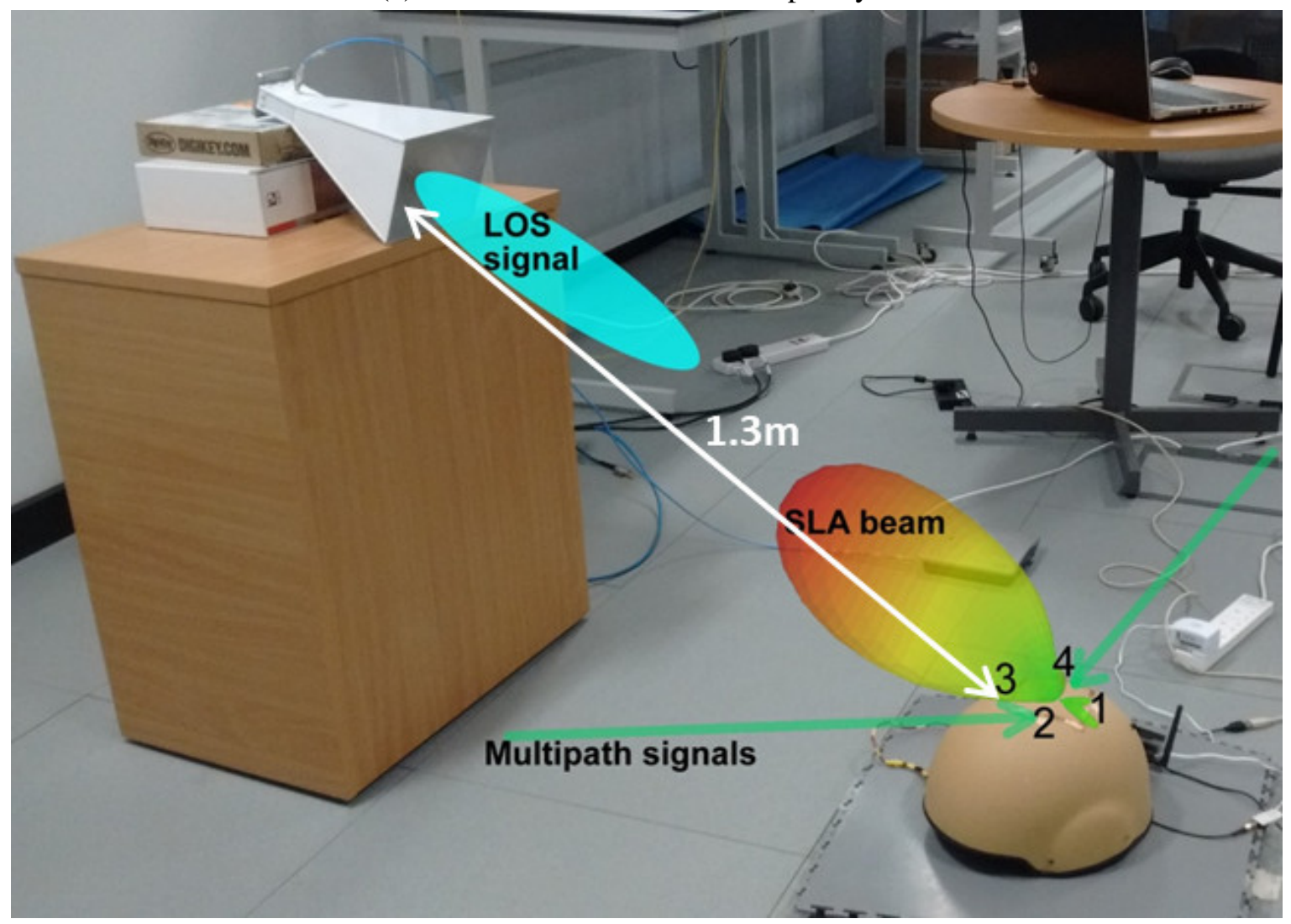

(b) The Raspberry Pi with SP4T switch controls and the WiFi Hotspot.

Fig. 5.8 The experimental setup. 


\subsection{Experimental setup}

Fig. 5.7 shows the complete configuration of the implemented beam-steering system. The transmitter is a horn antenna connected to the internet via a Cisco router (Cisco, 2006). The horn antenna provides a direct WiFi link to the receiver section and is placed on a wooden platform having a height of $70 \mathrm{~cm}$, as shown in Fig. 5.8b. The line of sight distance between HSLA and horn was 1.3 meters in the far field region.

The receiver section consists of a conformal beam-steering Hemispherical Square Loop Antenna (HSLA) integrated with a SP4T RF switch and a single board computer - Raspberry Pi. The Pi unit is attached behind a 7" touchscreen display which is powered by a USB (Universal Synchronous Bus) battery (Fig. 5.8a). The whole receiver section is placed on a turn table. For experimental purpose a near hemispherical Kevlar helmet was used as a substrate for the SLA.

The proposed system is designed to operate over $2.4 \mathrm{GHz}(802.11 \mathrm{~b} / \mathrm{g} / \mathrm{n}) \mathrm{WiFi}$ band. The center frequency of this band $(2.4 \mathrm{GHz}$ to $2.5 \mathrm{GHz})$ is $2.45 \mathrm{GHz}$ and it is selected as the test frequency throughout this chapter. The Pi was provided the WiFi functionality using a USB based WiFi transceiver (U1, 2004). The Omni-antenna for that transceiver was removed and the transceiver was directly connected to the SP4T RF switch. The switch then connects the transceiver to one of the four possible antenna ports. Based on which port was selected the antenna radiates a beam in one of the four possible spatial azimuth directions, as shown in Fig. 5.7.

\subsection{Throughput analysis}

The SP4T RF switch is connected to the HSLA using four semi-rigid coaxial cables, each having a length of $110 \mathrm{~mm}$. The main RF In/Out of the switch is connected to the USB WiFi adapter (transceiver) which is interfaced with Raspberry Pi where throughput is measured and examined. The switching control pins of the RF switch are connected to the GPIO pins of the Raspberry Pi that provide the necessary required biasing and switching control voltages. Raspberry Pi runs an intelligent beam-steering algorithm written using $\mathrm{C}$ called as the 'Electromagnetic Sense, Analyze and Lock' algorithm. It enables HSLA to lock always to a direction with which the Pi receives the highest RSSI signal value. This algorithm is shown in Fig. 5.9.

In the laboratory (Fig. 5.8), upon powering of the system the RF switch undertakes a fast sequential switch / scan (in $100 \mathrm{~ms}$ ) of the environment. This provides the four RSSI values for the signal received at the four HSLA ports from the WiFi channel being transmitted from 


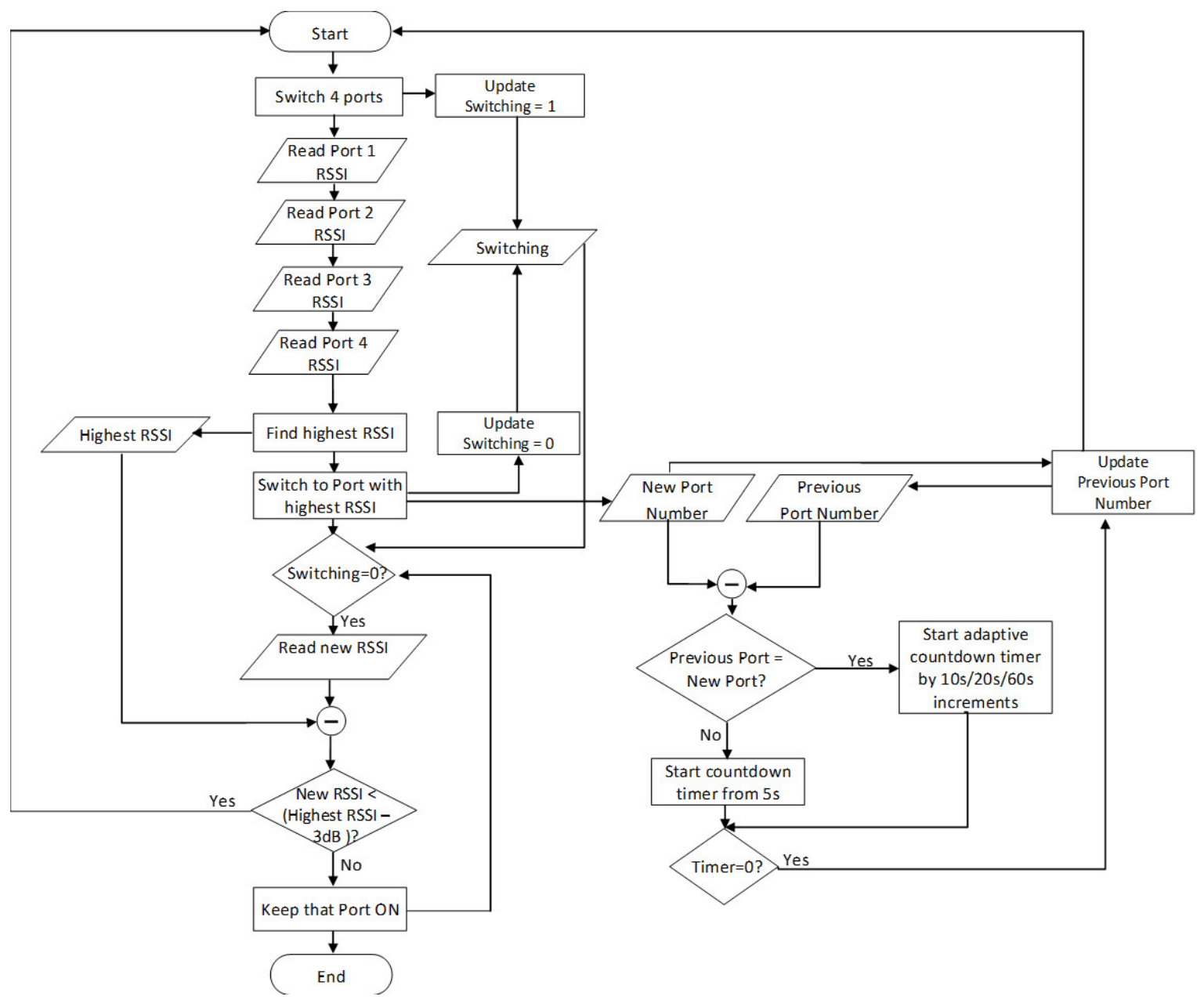

Fig. 5.9 The enhanced beam switching algorithm. 

applications

the horn antenna. The horn antenna is set to transmit a vertically polarized $\left(E_{\theta}\right) \mathrm{WiFi}$ signals at SSID (Service Set Identifier) '5G test' which is synchronized with the WiFi transceiver chip. The Pi compares the four RSSI values and locks the system to the port with highest value. 


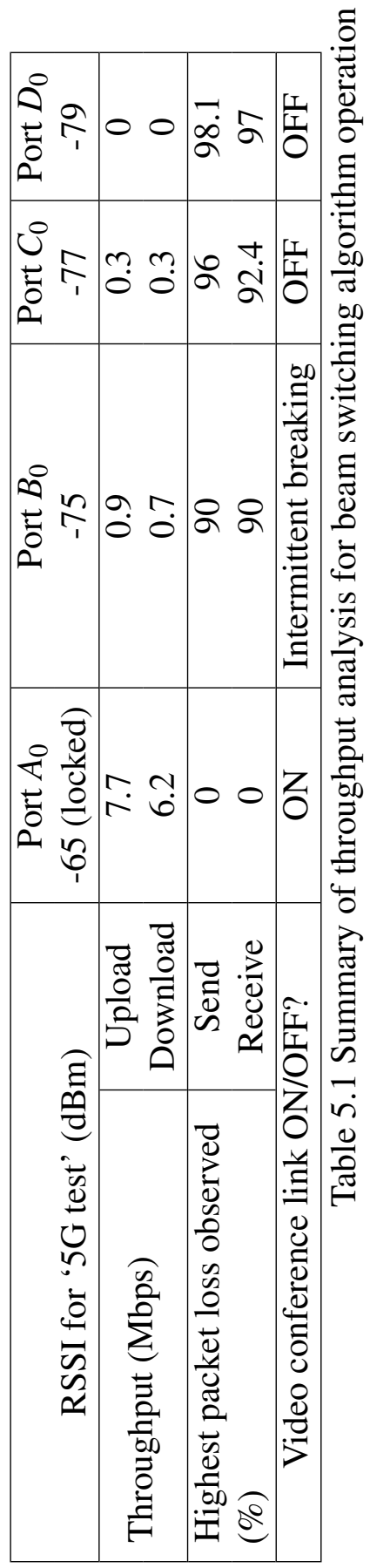




\section{HSLA Integrated with Raspberry Pi for a mobile user using high throughput applications}

Table 5.1 shows the four received RSSIs for the four ports and that the system is auto locked to the strongest RSSI port $A_{0}(-65 \mathrm{dBm})$. Thereafter, the system keeps monitoring the link. I set a dynamic setting to ensure that whenever a signal drops below a threshold the system undertakes a fresh scan.

For this work, I had set a setting such that 'if signal drops $3 \mathrm{~dB}$ below the current RSSI (in this instance $-68 \mathrm{dBm}$ ), it undertakes a new scan. The threshold to re-scan was dynamic and by using a slider on Graphic User Interface on the display it could have been changed easily for any desired value. Both transmitter and HSLA were moved around the laboratory and it was found that in all instances the algorithm always locked to the strongest RSSI signal direction.

I tested the system internet throughput on all four ports and found that there was a significant difference between the strongest and weakest signal ports. For instance, the strongest port $A_{0}$ provided an RSSI of $-65 \mathrm{dBm}$, a downlink of $7.7 \mathrm{Mbps}$ and an upload speed of $6.2 \mathrm{Mbps}$. This meant that port $A_{0}$ can fully support full HD duplex video link. In contrast, internet throughput measurements on other weaker ports $\left(B_{0}, C_{0}\right.$ and $\left.D_{0}\right)$ which were operating close to system noise floor of $-81 \mathrm{dBm}$ provided extremely weak data rates, even down to zero kbps for both upload and download speeds. For instance port $D_{0}$ RSSI was just $2 \mathrm{~dB}$ above noise floor and provided zero data speeds. Therefore, the beam steering enhanced the spectrum efficiency and its capacity for the fixed available WiFi bandwidth. These tests were done on a commercial throughput-measuring website.

In addition, the internet horn antenna link was not shared with any other user and was totally dedicated to this experiment. This test quantified the beam steering advantage in a real world of OFDM communications (which will also be the basis of 5G systems). It showed that a high Gain beam steering antenna enabled a device to lock to the direct / strongest signal, and that the device could sustain high definition video in an otherwise weak EM environment. Had the beam steering not been present in the same environment, in a worst case scenario the same device would have absolutely zero throughput. This advantage was further evident from the video test that was undertaken. It showed that a full $\mathrm{HD}$ was sustained by port $A_{0}$ with zero packet and frame loss. The other ports offered video conferencing with a weak intermittent service with $90 \%$ packet loss or absolutely no service at all. Thus, for a given weak EM environment beam steering was the difference between a good HD communication versus a very weak or no communication.

As would have been apparent from the system design, I only used a single antenna for both sensing and communication. Typically, an auxiliary sensing antenna is needed for electromagnetic environment sensing. In this case, I exploited the built in buffers and latency mitigation mechanisms of the TCP / IP WiFi network to ensure that no data is lost 
in the $100 \mathrm{~ms}$ scan duration. Note, while the switches can operate in nano seconds, a milli seconds scan duration was selected due to relatively slow time response of the USB WiFi adapter. Finally, as demonstrated in this work the algorithm works at the application layer that controls the RF switch and antenna patterns at physical layer. Therefore, in this work the entire middle protocol layers are untouched. Hence, the system can easily be retrofitted to existing non-adaptive communication systems to make them smarter and faster in weak EM environments.

\subsection{Conclusion}

A pattern adaptive Hemispherical Square Loop Antenna (HSLA) is integrated to Raspberry Pi single board computer. It is demonstrated that in a weak EM environment the adaptive antenna is capable of providing a throughput 7.7 Mbps and can sustain uninterrupted HD video conference. It was demonstrated that in a worst case scenario without having beam steering capability there would have been absolutely no communication ( 0 bps). The beam steering mechanism is implemented using a smart 'EM Sense, scan, analyze and lock' algorithm running on $\mathrm{Pi}$ in $\mathrm{C}$. The algorithm controls the beam direction of the SLA by using an RF SP4T switch linked to the SLA. The proposed system architecture used only a single antenna for both sensing and communications. Finally, the system had the application layer controlling the physical layer for a maximum possible RSSI. Thus, the entire middle protocol layers were untouched. 



\section{Chapter 6}

\section{Future Work}

\subsection{Introduction}

In this chapter I look at the work to be done in future for the beam steerable HSLA concept presented in Chapters 4 and 5. In the next section I study the adaptability of the HSLA for higher frequency bands (Ku band, K band and Ka band). I scale the 2.45 GHz HSLA antenna presented in Chapters 4 and 5 using its perimeter length and frequency as a guide to design a new planar SLA for these frequencies. The simulated planar SLA designs for the Ku band and the $\mathrm{K}$ band are presented next. Their 3D Gain patterns and the frequency response of their reflection coefficient is presented next. I also briefly discuss what feeding connectors and switching mechanisms, can be used at the higher frequencies for which the new planar SLAs are designed for.

Finally, I look at different applications for the beam switching algorithm discussed in Chapter 5. I next discuss how the use of the DoA estimation metric and algorithm mentioned in Chapter 3, can be applied for the case of a 4 beam switching HSLA. I then discuss how the switching metric of the algorithm presented in Chapter 5 can be replaced with an alternative, more accurate metric such as SNR in order to improve the BER performance of multimedia or high bandwidth applications. I then look at the usefulness of calculating and implementing the optimal switching thresholds for the SSC and SEC switching scheme algorithms, as presented in the Literature Review Section (Chapter 3), for the case of the HSLA presented in Chapters 4 and 5.

This chapter concludes by reviewing the main ideas discussed in the previous sections. It also reviews the preliminary work done for implementing the HSLA for higher frequency bands, as discussed in the earlier sections. 


\subsection{Planar SLA for Ku band}

The conformal HSLA presented in Chapter 4 for sensor node applications, had a perimeter length of $\mathrm{C}=384 \mathrm{~mm}$ (i.e $3.1 \lambda_{0}$ or $4.7 \lambda_{g}$ at $2.45 \mathrm{GHz}$ ) where $\lambda_{0}$ is the free space wavelength and $\lambda_{g}$ is the guide wavelength respectively. The guide wavelength $\lambda_{g}$ is calculated as follows:

$$
\lambda_{g}=\frac{\lambda_{0}}{\sqrt{\frac{\varepsilon_{r}+1}{2}}}
$$

The previous work done for the SLA carried perimeter lengths of $\mathrm{C}=120 \mathrm{~mm}\left(2.67 \lambda_{g}\right)$ at $4.7 \mathrm{GHz}$ (Deo et al., 2010) and $2.68 \lambda_{g}$ at $4.5 \mathrm{GHz}$ (Pal et al., 2008). The DBCCSLA proposed by Pal et al. (2014a,b) have perimeter lengths of $3.55 \lambda_{g}$ (at $3.1 \mathrm{GHz}$ ) and $3.63 \lambda_{g}$ (at $3.63 \mathrm{GHz}$ ) for the two frequency bands due to the dual loops. The capacitively fed single SLA reported by Pal et al. (2012b) carried a loop perimeter of $3.47 \lambda_{g}$ at $5.2 \mathrm{GHz}$.

Using the above loop perimeter lengths $\left(C_{2.45 \mathrm{GHz}}=384 \mathrm{~mm}\right)$ and guide wavelengths $\left(4.65 \lambda_{g}\right)$ as a guide, the feasibility of scaling the SLA prototype built in Chapter 4, to design a new SLA for the $\mathrm{Ku}$ band ( $12 \mathrm{GHz}$ to $18 \mathrm{GHz}$ ) was explored. For this preliminary study, a planar SLA (not HSLA) for $12 \mathrm{GHz}$ operation was designed. Its perimeter $C_{12 \mathrm{GHz}}$, was calculated as:

$$
C_{12 \mathrm{GHz}}=4.65 \lambda_{g}=4.65 \times 19.37 \mathrm{~mm}=90.09 \mathrm{~mm}
$$

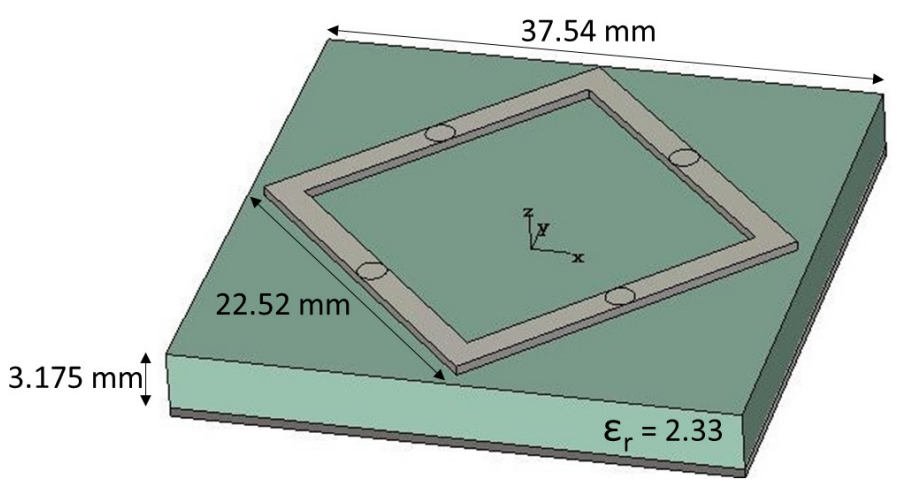

Fig. 6.1 Design of $4.65 \lambda_{g}$ SLA.

The design of the SLA is shown in Fig. 6.1. Its substrate is made of Rogers RT5870 duroid material $\left(\varepsilon_{r}=2.33\right)$. It has substrate thickness of $3.175 \mathrm{~mm}$ and substrate width of $37.54 \mathrm{~mm}$. The SLA has width of $22.52 \mathrm{~mm}$ in each side. 


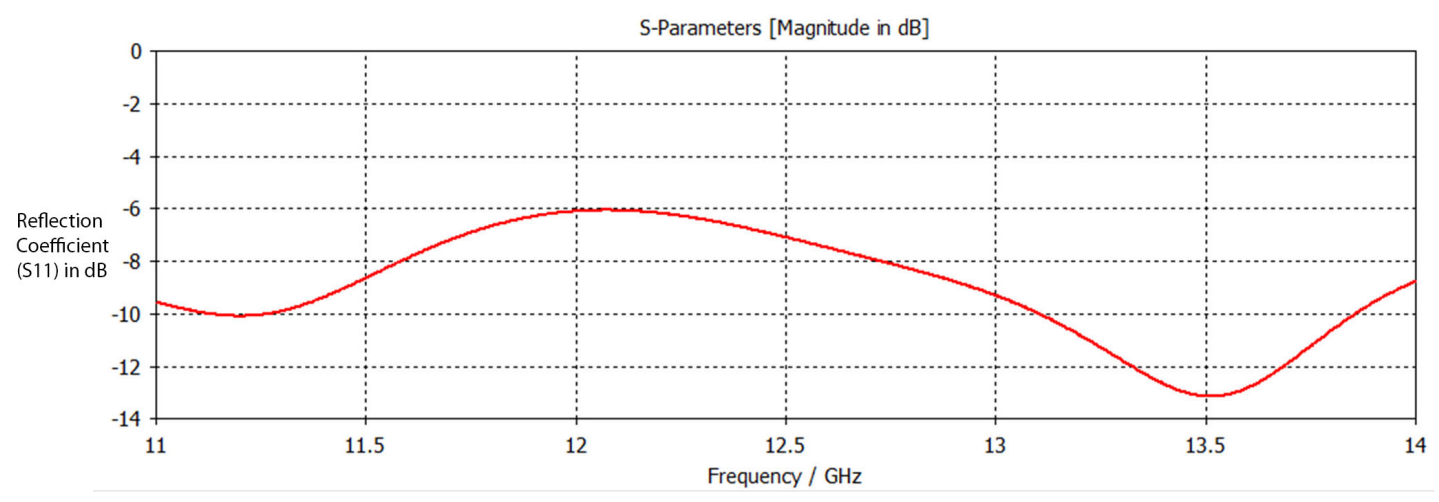

(a) Frequency response of Return Loss Co-efficient.
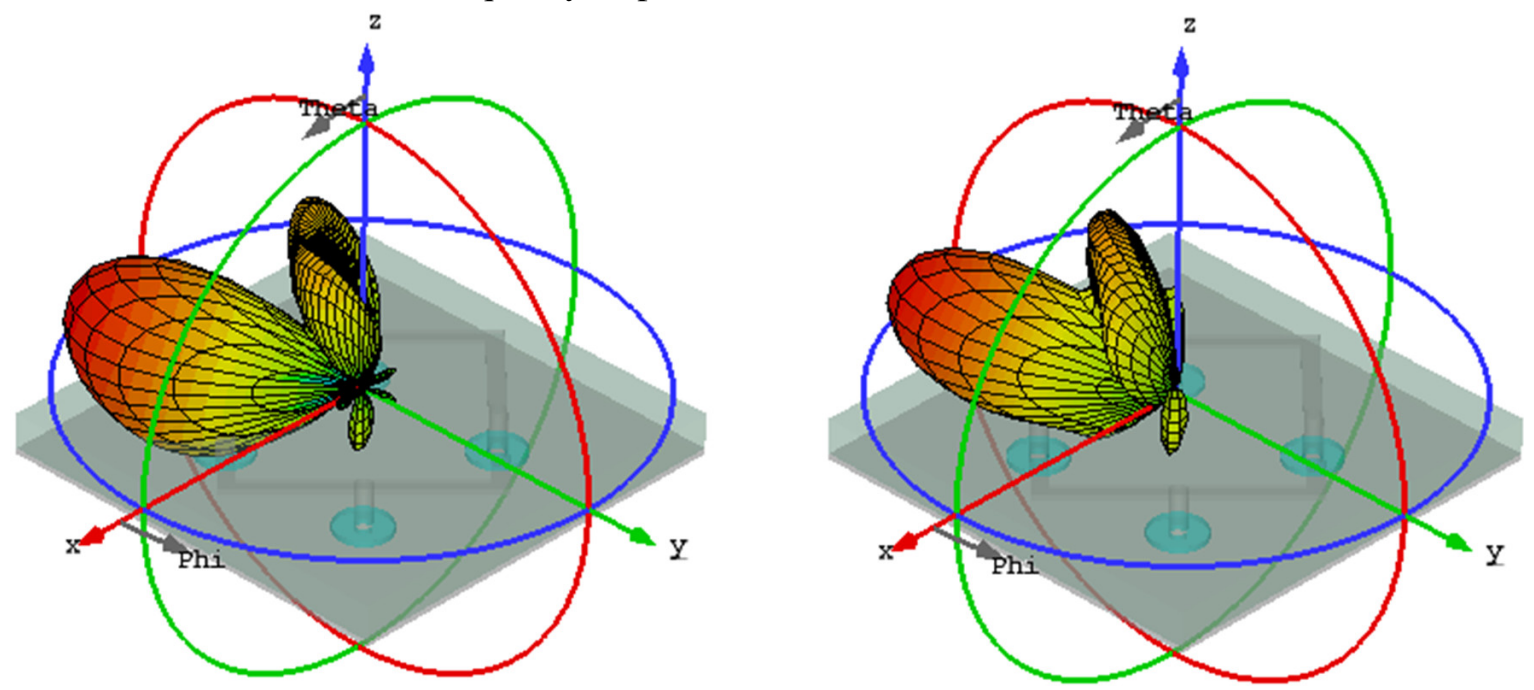

(b) $3 \mathrm{D}$ radiation pattern of $4.65 \lambda_{g}$ SLA at $12 \mathrm{GHz}$ (Left) and $13 \mathrm{GHz}$ (Right).

Fig. 6.2 Simulated results of the $4.65 \lambda_{g}$ SLA designed for $12 \mathrm{GHz}$. 


\section{Future Work}

The simulated results are shown in Fig. 6.2. Fig. 6.2a shows the frequency response of its return loss coefficient ( $S_{11}$ graph). Its reflection coefficient is less than $-10 \mathrm{~dB}$ from $13.1 \mathrm{GHz}$ to $13.85 \mathrm{GHz}$ (750 MHz impedance bandwidth). However, its radiation patterns shown in Fig. $6.2 \mathrm{~b}$ (Left) contain a strong lobe in the $+\mathrm{Z}$ axial direction. Therefore, this compromises the SLA's multipath mitigation characteristics that were discussed in Chapters 3, 4 and 5. Fig. $6.2 \mathrm{~b}$ (Right) shows its $3 \mathrm{D}$ radiation pattern at $13 \mathrm{GHz}$, in which the lobe in the $+\mathrm{Z}$ axis direction is reduced and its directivity of the main lobe (at $\phi=315^{\circ}$ direction) is $9.1 \mathrm{~dB}$.

These preliminary simulated results are promising and indicates that this SLA can be scaled for any frequency by carefully choosing the circumference C. More work is needed to optimize the substrate width and the width of the SLA's loop, in order to create a highly directive tilted beam pattern, similar to that of the HSLA presented in Chapters 4 and 5.

For this design a loop perimeter of $C=4.65 \lambda_{g}$ was chosen. This was the highest perimeter length achieved so far (HSLA in Chapter 4) for highly directive radiation pattern generation, among all the SLA designs studied upto now. However, according to the preliminary results observed for this planar SLA at $12 \mathrm{GHz}$, a compromise needs to be made between increasing the loop perimeter in terms of $\lambda_{g}$ and achieving satisfactory radiation patterns as in previously studied SLAs. These aspects need to be further studied.

\subsection{Planar SLA with HHIS for K band}

I next designed a planar SLA on a HHIS for $25 \mathrm{GHz}$ operation. The design of the antenna is similar to the one reported in Deo et al. (2010). It has been scaled down in size to suit $25 \mathrm{GHz}$ operation by proportionally adjusting its dimensions by a scaling factor of 5.32 $\left(\frac{25 \mathrm{GHz}}{4.7 \mathrm{GHz}}\right)$. This method of scaling is somewhat different to the criteria used in the previous section (equation 6.2).

The designed planar SLA's loop width is $6.3 \mathrm{~mm}$ and it has thickness of $1.27 \mathrm{~mm}$. A Rogers RO4350B duriod dielectric material was chosen as its substrate. A HHIS consisting of a $6 \times 6$ array of conducting square patches of width $1.65 \mathrm{~mm}$ with gaps of $0.282 \mathrm{~mm}$ between them, was embedded in the substrate. The patches on the outer perimeter have vias at the center connecting them to the ground plane at the bottom of the substrate similar to the HHIS design in Deo et al. (2010). The substrate thickness has height $3.18 \mathrm{~mm}$ above the HIS and $1.52 \mathrm{~mm}$ below the HIS surface. This design is shown in Fig. 6.3.

The preliminary results of the frequency response of the reflection coefficient and the simulated 3D radiation pattern are shown in Fig. 6.4. From the reflection coefficient seen in Fig. 6.2a there is very close impedance matching to $50 \Omega\left(S_{11}<-10 \mathrm{~dB}\right.$ criteria) at 23.7 $\mathrm{GHz}$ and $25.8 \mathrm{GHz}$. From the 3D directivity pattern presented in Fig. $6.4 \mathrm{~b}$ it can be seen that 

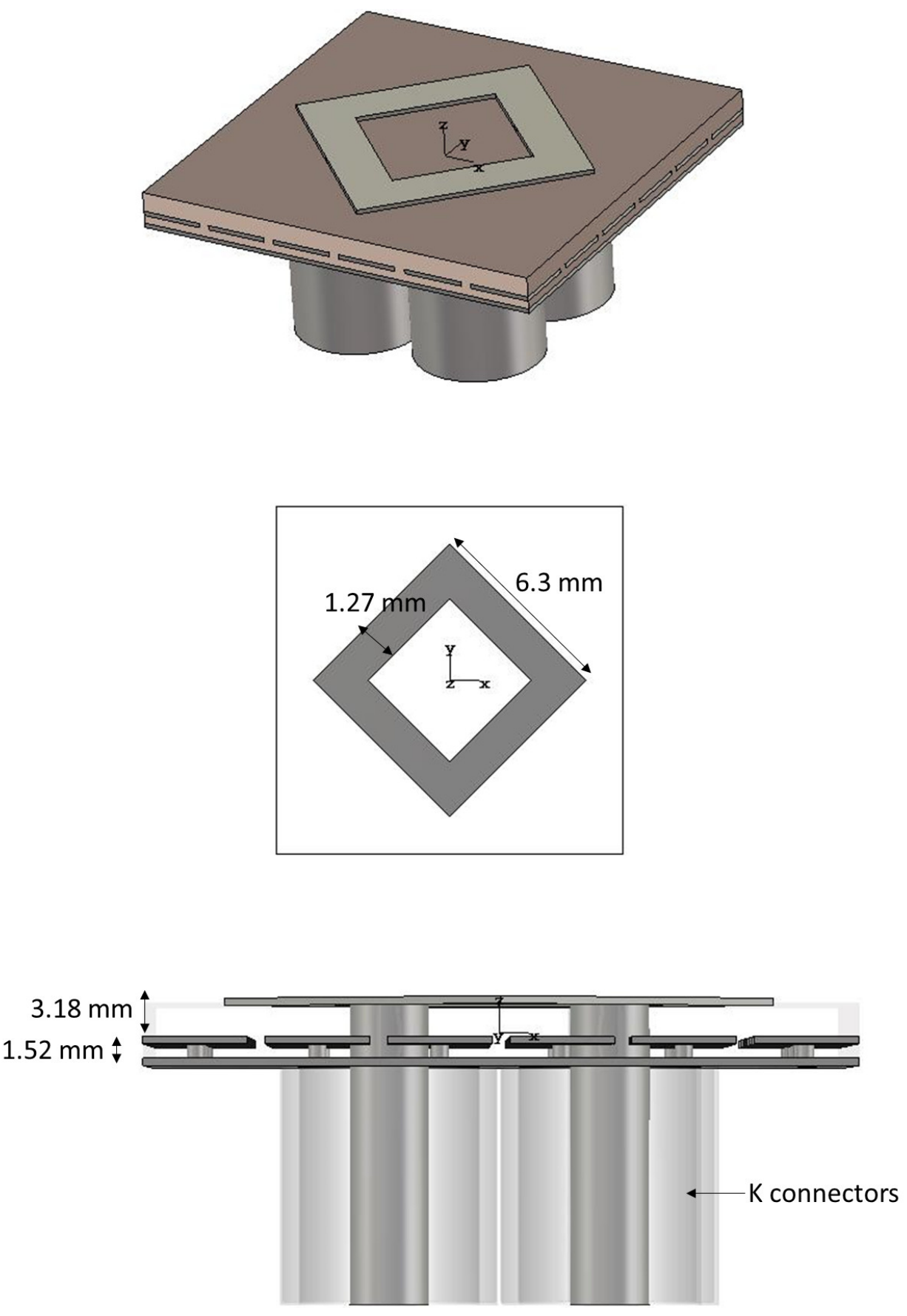

Fig. 6.3 Design of scaled SLA with HHIS for 25 GHz. Perspective view (Top), Top view (Middle) and Side view showing the HHIS (Bottom). 


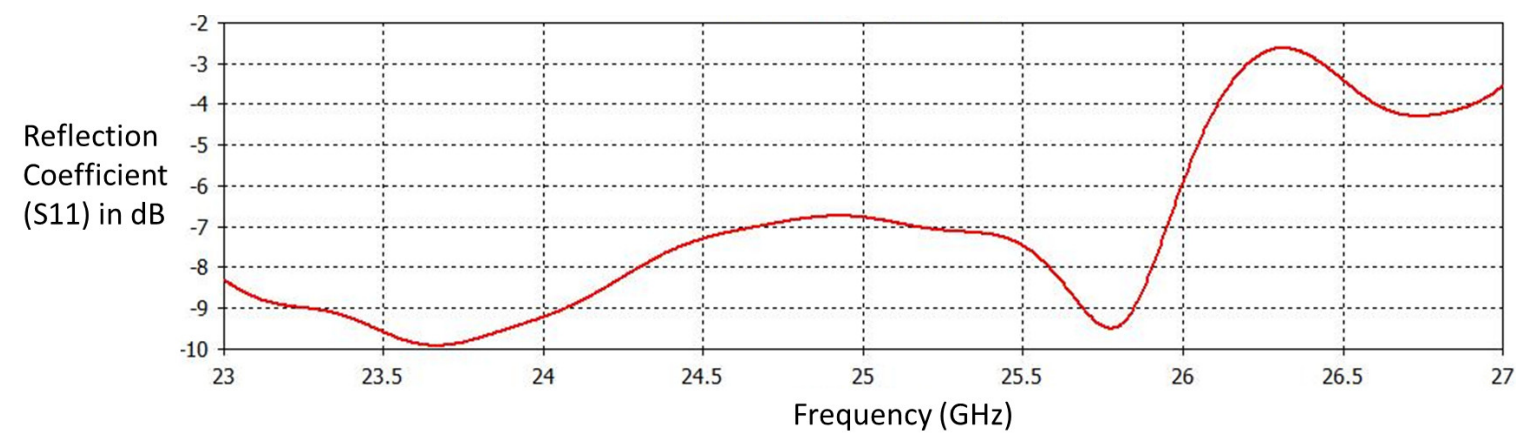

(a) Frequency response of Return Loss Co-efficient.
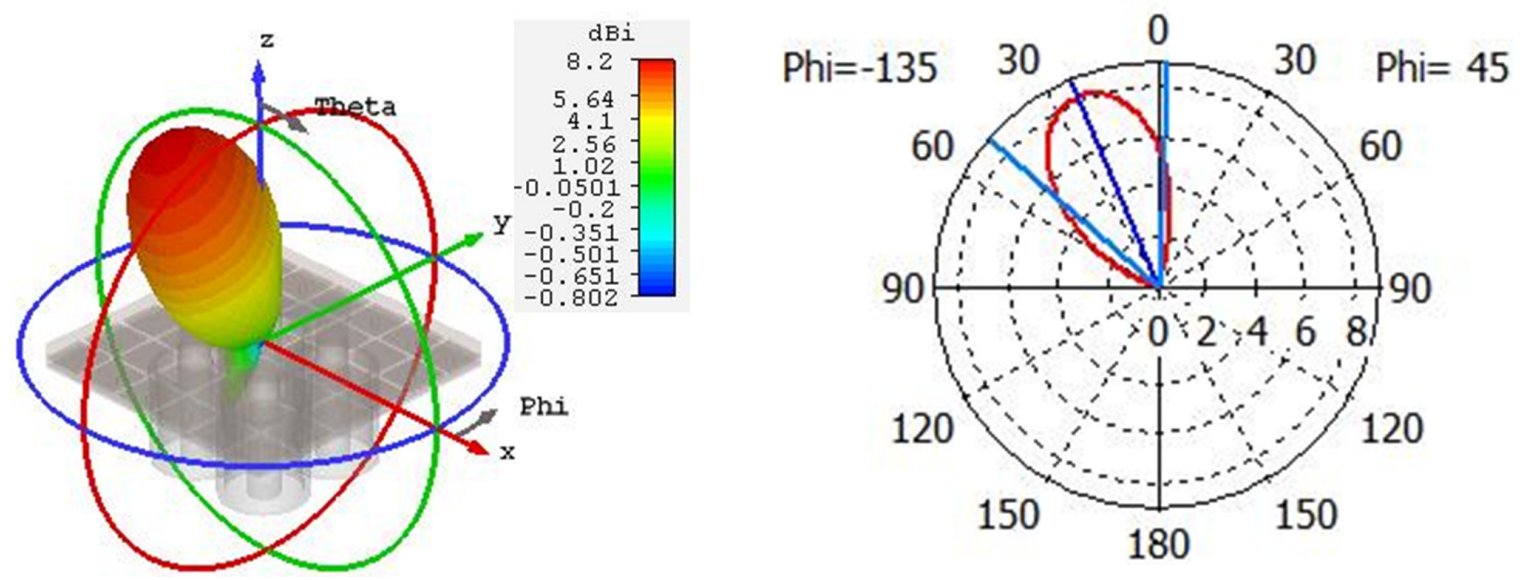

(b) 3D Directivity pattern of SLA with HHIS at $25 \mathrm{GHz}$ (Left) and its vertical cut of $E_{\theta}$ at $\phi=$ $45^{\circ}$ (Right).

Fig. 6.4 Simulated results of the SLA with HHIS for $25 \mathrm{GHz}$. 


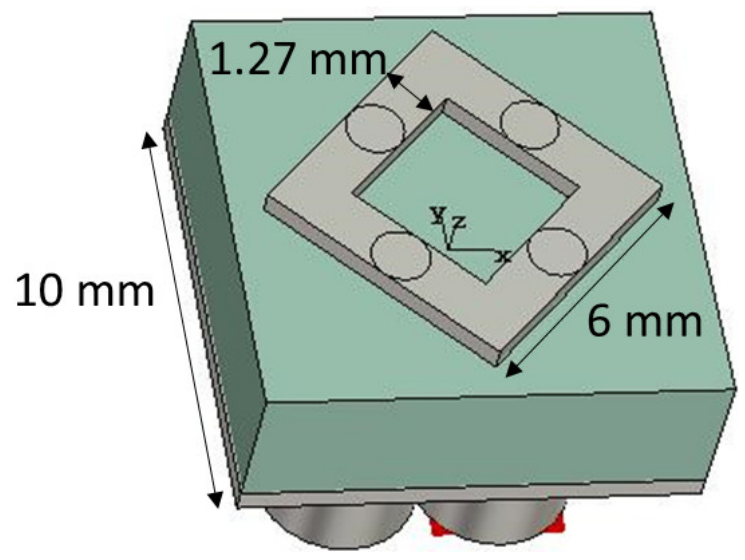

Fig. 6.5 Perspective view of the designed SLA scaled for $30 \mathrm{GHz}$.

this SLA with HHIS creates a $8.2 \mathrm{~dB}$ directive pattern at $\phi=315^{\circ}$ and $\theta=23^{\circ}$. This beam tilt is significantly less than the tilt of the previously studied SLA with HHIS $\left(\theta=45^{\circ}\right)$ at 4.7 GHz (Deo et al., 2010).

Therefor,e a careful study of this preliminary design needs to be conducted in order to optimize its dimensions to suit its new frequency of operation at $25 \mathrm{GHz}$ to create a more tilted beam. Furthermore, a study needs to be conducted on the feasibility of building a conformal HHIS for this frequency band, in order to mount this antenna on conformal land or aerial vehicular platforms.

\subsection{Planar SLA for Ka band}

After the encouraging results shown by the SLA with HHIS designed for $25 \mathrm{GHz}$ in the previous section, I next explored the possibility of designing such a beam steerable SLA for higher frequency bands such as the Ka band $(26 \mathrm{GHz}-40 \mathrm{GHz})$. Having such high frequency antennas would be useful for testing them with high-resolution close-range targeting radars for military applications.

The antenna's dimensions presented by Pal et al. (2008) were used as a guide in designing a new antenna for $30 \mathrm{GHz}$ (Ka band) operation. The dimensions from the previous $4.5 \mathrm{GHz}$ antenna were scaled while keeping the ratio of the SLA's diagonal and the substrate width, the same. This ratio of $30 \sqrt{2}: 50$ was fixed for the new design. Therefore, a new SLA was designed whose square loop arms has $6 \mathrm{~mm}$ width and $1.27 \mathrm{~mm}$ thickness. Its substrate has width $10 \mathrm{~mm}$. This design shown in Fig. 6.5 does not have a HIS as in the $25 \mathrm{GHz}$ (K band) SLA (Fig. 6.3) described in the previous section. 


\section{Future Work}

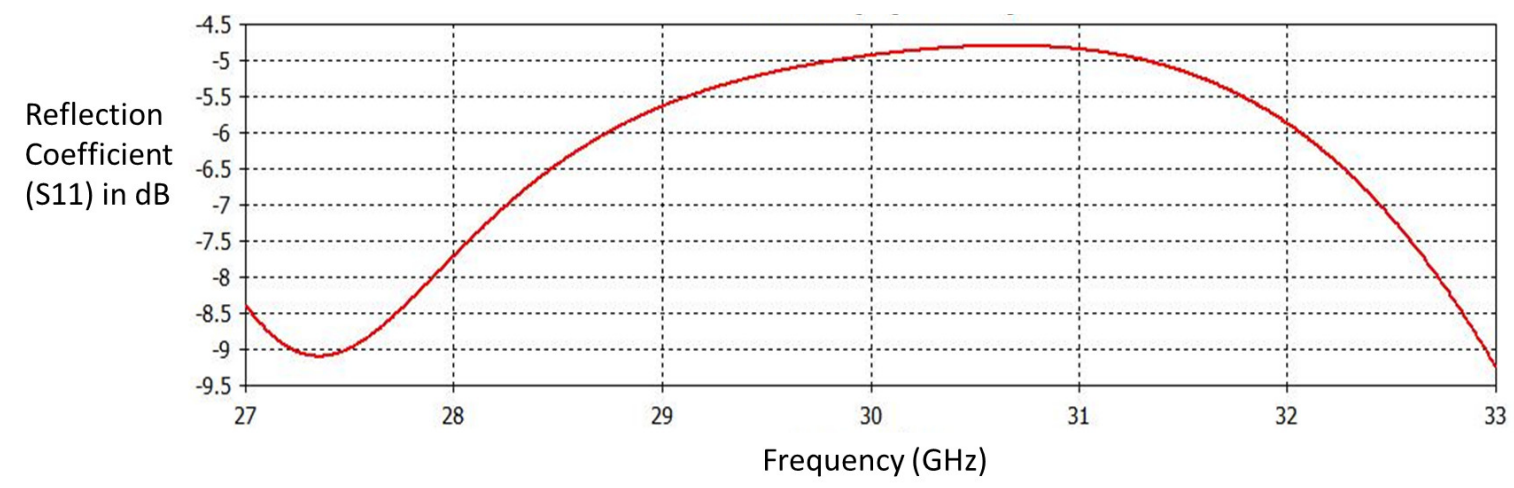

(a) Frequency response of Return Loss Co-efficient.
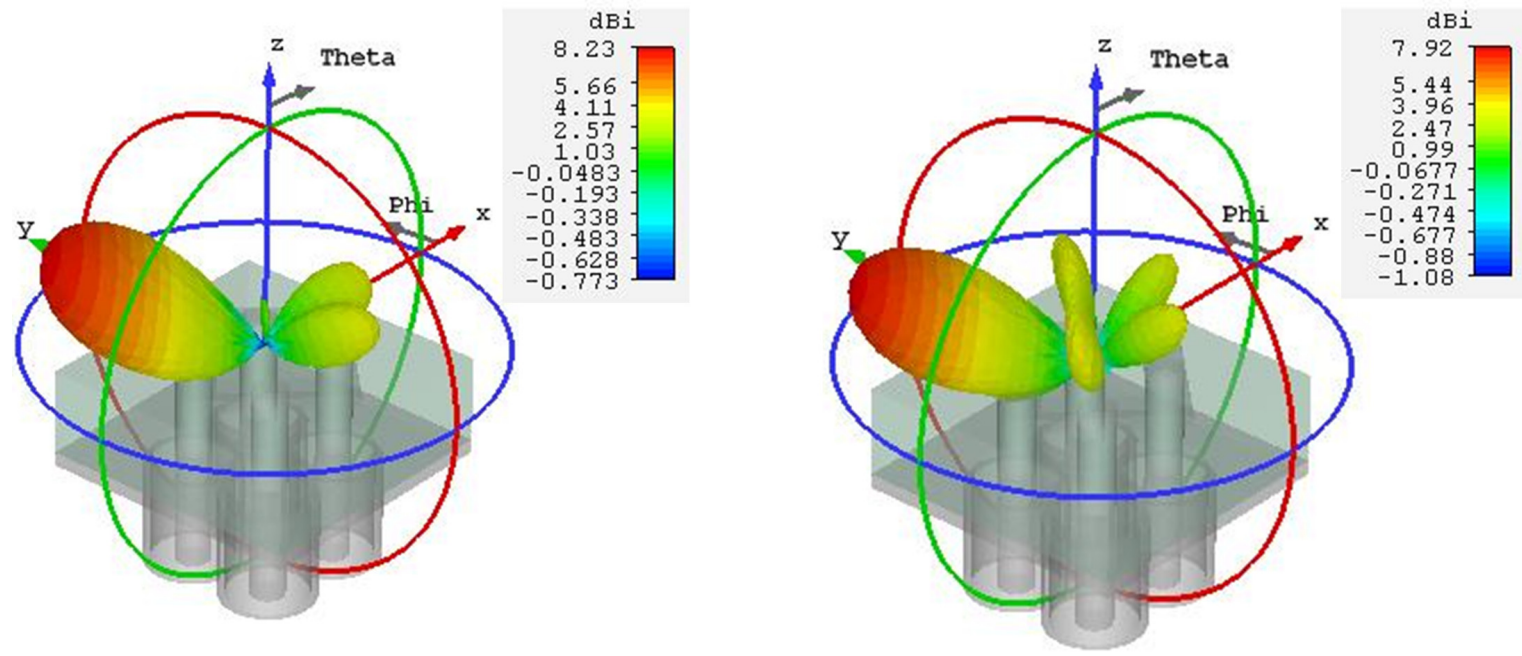

(b) 3D directivity pattern of SLA with HHIS at $30 \mathrm{GHz}$ (Left) and at $32 \mathrm{GHz}$ (Right).

Fig. 6.6 Simulated results of the SLA for $30 \mathrm{GHz}$. 
The simulated results for the SLA are shown in Fig. 6.6. The frequency response of the return loss coefficient $\left(S_{11}\right)$ shown in Fig. 6.6a shows good impedance matching $\left(S_{11}<\right.$ $-9 \mathrm{~dB}$ ) at around $27.5 \mathrm{GHz}$ and beyond $33 \mathrm{GHz}$. However, the impedance matching at the designed frequency of $30 \mathrm{GHz}$ is poor $\left(S_{11} \approx-5 \mathrm{~dB}\right)$.

The 3D directivity pattern of the designed SLA is shown in Fig. 6.6b. The pattern at 30 $\mathrm{GHz}$ (Fig. 6.6b(Left)) shows a highly directive main beam in the $\phi=135^{\circ}$ azimuth direction which is tilted in the elevation plane. However, the pattern also contains a minor lobe in the opposite azimuth direction $\left(\phi=-45^{\circ}\right)$. There is also a minor lobe along the $+\mathrm{Z}$ axis. However, this is approximately $-8 \mathrm{~dB}$ lower than the main lobe direction. The $3 \mathrm{D}$ radiation pattern for $32 \mathrm{GHz}$ shown in Fig. 6.6b(Right) shows that the pattern deteriorates further at higher frequencies although its impedance matching improves beyond $33 \mathrm{GHz}$.

Therefore, by looking at the simulated results of the $30 \mathrm{GHz}$ SLA for Ka band shown in Fig. 6.6, I can conclude that more work needs to be done to design this antenna for higher frequency bands such as the Ka band. In particular, its input impedance bandwidth needs to be matched closer to $50 \Omega$ at the desired frequency. Furthermore, its dimensions need to be optimized to achieve more directive patterns without side lobes and minor lobes in the opposite direction, in order to have more efficient multipath mitigation, avoid signal jamming and interference from other directions and achieve longer communication range in its main lobe's direction. The effects of making this antenna conformal for vehicular platforms or satellites, need to be studied as well.

\subsection{DoA estimation using 4 beam SLA}

The DoA estimation algorithm by Sanchis et al. (2002) was briefly introduced in the Literature Review under Section 3.11. This algorithm uses a 3-beam antenna switching scheme to estimate a switching estimator, $d$. Equation 3.4 describes the estimation criteria for $d$ which compares the power between two adjacent antenna beam positions.

The HSLA introduced in Chapter 4 and Chapter 5 have 4 switched beam positions. I therefore, can apply the DoA estimation algorithm presented by Sanchis et al. (2002) for this HSLA. The HSLA presented in this thesis has the advantage of covering all 4 spatial quadrants in the $0^{\circ}<\phi<360^{\circ}$ azimuth range. This is especially useful if the base station and the receiver are both moving at a fast pace, as in the case of the air-to-ground (or vice versa) or air-air military communication. In such a scenario, the receiver's position could change in a fast enough manner such that, all $360^{\circ}$ azimuth range needs to be scanned to search the new location of the user. The HSLA is thus a good candidate for employing a 


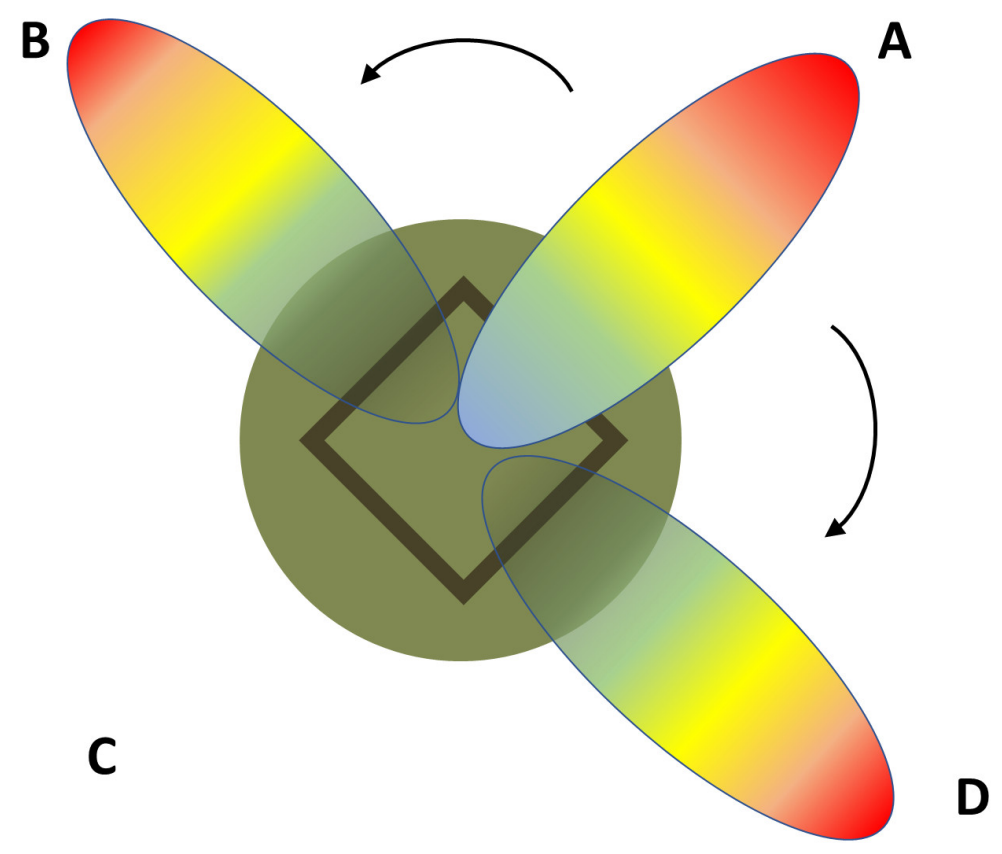

Fig. 6.7 Illustration of beam switching sequence of HSLA for DoA estimation algorithm.

new DoA estimation algorithm if the receiver or the transmitter move quickly in the azimuth direction.

The proposed beam scanning that can be employed in such an algorithm is shown in Fig. 6.7. It scans the two adjacent positions as well as the current position to measure the received signal power. It then computes the switching estimator-d based on the 3 signal powers for the current position as well as the previous and the next adjacent positions. For example, in Fig. 6.7, the current position is A and if its previous position is B, then its next adjacent position will be $\mathrm{D}$. Then the switching estimator $\boldsymbol{d}$ can be computed according to equation 3.4 presented earlier, after the scanning is completed as follows:

$$
d=\frac{\left(P_{D}-P_{B}\right)}{P_{A}}
$$

The zero crossing of the moving estimator $\boldsymbol{d}$ is then considered to gauge the movement of the mobile subscriber as explained in Section. 3.11. If $\mathbf{d}=0$, it is assumed that the user has remained in the sector covered by beam position A. If $\boldsymbol{d}$ has crossed from negative to positive it is assumed that the mobile subscriber has moved to position D from A. If $\boldsymbol{d}$ has crossed from positive to negative, it is assumed that the mobile subscriber has moved from sector covered by beam position A to sector B.

However, since the HSLA has 4 beam positions, there is more than 2 two neighbours for each positions as it is possible for the mobile subscriber to move to the positiona directly 
opposite the current antenna beam position (i.e from A to C) during communication in a fast moving scenario. Therefore, the method mentioned by Sanchis et al. (2002) will need to be modified to include power obtained from all 3 neighbouring positions ( $B, C$ and $D)$. This would potentially include extra beam switching time for a third position (C) in Fig. 6.7. In addition, the overhead added to the communication in transmitting the preamble will need to increase by a factor of $50 \%$ due to this extra beam switching position.

Therefore, for future work I propose studying the effects of having 3 neighbouring positions in the DoA estimation algorithm proposed by Sanchis et al. (2002) while modifying the equation 6.3 to compute the switching estimator $\boldsymbol{d}$ in order to take it into account.

\subsection{SSC and SEC schemes using SNR for HSLA}

The beam switching algorithm used for the HSLA in Chapter 4 and 5 follows a hybrid of the SC and SEC schemes. It first scans all 4 ports of the HSLA and switches to the port with the highest RSSI (SC scheme). It then continues to monitor the switching criteria (RSSI) against a pre-set threshold (SEC scheme) and when the switching criteria is met, conducts another scan of the 4 ports and switches to the port with the highest RSSI (SC scheme again).

The SC, SSC and SEC switching schemes were introduced earlier in Chapter 3. In view of the algorithm developed, I propose to use a different switching metric such as the SNR in order to conduct the switching. Several work has previously been reported that investigated the usefulness of using SNR as a switching metric to improve the BER performance (Moradi et al., 2012; Zhao et al., 2015).

In order to read and monitor a switching metric such as the SNR, a different approach than the monitoring of the RSSI described in Chapter 4 and 5 is necessary. The SNR can be computed by employing signal processing at the receiver level. This will require the use of a custom made RF receiver circuit with DSP modules for the required frequency. Another approach is to use a combination of a physical RF circuit along with a software defined radio (SDR) such as the GNU radio (GNU Radio Website, 2016) in order to obtain this information. Finally, a packet sniffing method such as libtins (Libtins Library, 2015) can be used if the data packets can be accessed from the device in which the algorithm is run. 


\subsection{Implementation of an adaptive optimal switching thresh- old for HSLA}

I next propose to conduct a further study to implement a moving switching threshold for HSLA. As mentioned in Chapter 3, the rate of switching between the antenna elements is a crucial factor in reaching the optimal BER performance of a SEC scheme (Jataprolu et al., 2011). Frequent switching will cause problems at the receiver end when switching is sometimes done before the channel estimation is completed for a particular antenna. This would lead to channel estimation errors. On the other hand, setting the switching threshold too low would mean a less than ideal antenna beam position would be selected and persisted with, despite having better options available. This would lead to non-optimal use of the communication channel bandwidth as BER will rise due to high noise in the chosen channel. Therefore, carefully selecting an optimal switching threshold that takes into account the channel's SNR is necessary.

The beam switching algorithm implemented for the HSLA in Chapter 5 is very similar to the distributed switch-and-examine combining with threshold-based relaying (DSECT) proposed by Herath et al. (2012), although it was proposed for a amplify-and-forward (AF) switch combining scheme. In this distributed SEC scheme, The relay antenna which maximizes the SNR at the receiver is selected and then its SNR is continuously monitored and compared against a pre-set threshold. Swaminathan et al. (2016) observes through simulated numerical experiments that, as the average value of the SNR increases, the optimal threshold that minimises the BER also increases. Hence, it is necessary to have a moving threshold that take into account the improvement in the SNR as well.

Therefore, I propose to explore the possibility of implementing a moving threshold that takes into account the changes in the average SNR of a channel, observed over a period of time.

\subsection{Conclusion}

In this chapter I first looked at ways of scaling the HSLA built in Chapter 4 and 5 for higher frequency bands such as the Ku band, $\mathrm{K}$ band and the Ka band. The HSLA in Chapter 4 and 5 had perimeter lengths of $384 \mathrm{~mm}$ (i.e $4.65 \lambda_{g}$ ). A new SLA with perimeter length $4.65 \lambda_{g}$ of for the $\mathrm{Ku}$ band $(12 \mathrm{GHz})$ was designed in Section 6.2. The simulated SLA has fairly good impedance matching for the simulated frequency bandwidth $\left(S_{11}<-6 \mathrm{~dB}\right)$. However, the radiation patterns generated consisted of an axial lobe in the $+\mathrm{Z}$ direction. Therefore more work is need to be done to optimise this preliminary design was identified. 
In the next section, I designed a $\mathrm{K}$ band antenna for $25 \mathrm{GHz}$. The SLA with HHIS presented by Deo et al. (2010) was used as a guide. Its dimensions, including the HHIS parameters were scaled down by a factor of $5.32\left(\frac{25 \mathrm{GHz}}{4.7 \mathrm{GHz}}=5.32\right)$. This newly designed SLA with HHIS at $25 \mathrm{GHz}$ had a reasonably good impedance bandwidth from $23.4 \mathrm{GHz}-26 \mathrm{GHz}$ $\left(S_{11}<-6 \mathrm{~dB}\right)$. The generated radiation pattern at $25 \mathrm{GHz}$ consisted of a directive main lobe with no significant side lobes in other directions. However, its beam tilt was not satisfactory. Therefore, work for the future was identified in optimising this SLA with HHIS to produce a more tilted beam.

The Ka band (30 GHz) SLA antenna designed in the next section showed that it had good impedance matching at around $27.4 \mathrm{GHz}$ and beyond $33 \mathrm{GHz}$ (not simulated). Its 3D directivity pattern showed a main lobe with a good tilt in addition to fairly significant minor lobes in the opposite azimuth direction. Therefore, the dimensions of this antenna needs to be optimized to produce better radiation patterns as well as produce a good impedance bandwidth around the designed frequency of $30 \mathrm{GHz}$.

I next considered how to implement the DoA estimation algorithm proposed by Sanchis et al. (2002) for this 4 beam HSLA. An advantage of using this HSLA is that it can provide DoA estimation in a complete $360^{\circ}$ azimuth range. It was identified that the algorithm previously discussed by Sanchis et al. (2002) needs to be modified in order to consider 3 adjacent beam positions instead of the 2 adjacent beam positions discussed in literature.

The penultimate section of this Chapter discussed the feasibility of implementing an SNR based SSC and SEC schemes instead of the currently used RSSI based SEC scheme. The final section discussed the implementation of a moving-optimal threshold for the SEC scheme employed in Chapter 4 and 5. 



\section{Chapter 7}

\section{Conclusion}

\subsection{Introduction}

In this chapter I conclude the main body of this thesis by summarising the main research ideas and findings presented in each of the previous chapters in this thesis. The following sections of this chapter are organised as follows:

The next section of this chapter discusses how the research idea was introduced in Chapter 1: Introduction. Then it highlights the important theoretical aspects and physical phenomena relevant to the research problem outlined in Chapter 2: Background. It also summarises the problem statement and the knowledge gap identified in Chapter 2. It then summarises the main findings presented in Chapter 3: Literature Review.

The next two sections of this chapter summarise the main analytical (simulated) and experimental research work done in designing a HSLA and its beam steering algorithm run on two hardware platforms, as a solution to the problem statement highlighted in Chapter 2. It first describes how the HSLA was designed after a study conducted on making a planar SLA conformal. Then it summarises how the prototype of the HSLA was used as a body wearable antenna running a beam switching algorithm in a multipath environment. It then discusses how its communication performance in a high throughput application was improved using the hybrid beam switching scheme employed by the algorithm, running on a portable hardware platform.

The final section of this chapter summarises areas for further development identified for this antenna in Chapter 6: Future Work. It also summarises how the HSLA's beam switching algorithm can be improved by applying other techniques. It then concludes by reviewing the contribution to knowledge contained within this thesis and how the solution to the problem statement was achieved. 


\section{Conclusion}

\subsection{Thesis summary}

In the first chapter I outlined the motivation for the research study conducted and presented in this thesis. I then discussed and identified certain research areas that would have an impact on the future of mobile and defense communication. In particular, I looked at the usefulness of implementing beam steering and user tracking algorithms in a much more robust way than is currently used, in order to provide a continuous high throughput data link for a fast moving user and/or fast moving BS scenario.

Chapter 1 also briefly discusses the hardware requirements and the characteristics of the antenna required for realising a communication link running such an algorithm. It then discusses the potential advantages over conventional antennas in implementing such a system. The advantages of using a highly directive antenna beam pointed towards the moving BS or the MS were identified. It was stated that such an antenna system would have several additional advantages in a defense scenario such as range extension, more efficient use of battery power, more clear communication leading to better battlefield decision etc. Finally, in Chapter 1 I provided an overview of the organisation of this thesis. It provided a brief introduction to the knowledge and findings presented in each of the subsequent chapters.

In Chapter 2 I first discussed several physical phenomena that occur during wireless communication, that give rise to several problems in signal communication. Multipath mitigation, signal jamming and interference caused by omni-directional antennas and communication range extension and efficient use of battery power when using directive antennas were discussed in detail.

First, multipath propagation was defined and described briefly. Then I looked at the effects of multipath in wireless communication such as ISI, DoA estimation errors, partial/total cancellation, errors in time delay estimate in DLL and reduced effective bandwidth. I then discussed in detail several known methods used in multipath mitigation in order to overcome these problems. They were broadly categorised into statistical filtering methods (MAP, MMSE, MLE and JMS), correlation and deconvolution methods using DLLs, signal coding methods and use of high Gain antennas.

I then studied the important aspects of high gain antennas. I defined Gain, Directivity and antenna efficiency which are important aspects used to describe the performance of a directive antenna. I also briefly looked at the theory behind using antenna arrays to improve Gain, conduct beam scanning using phase shifting and increasing signal reach.

I next looked at the impact on battery power of portable radio units carried by mobile infantrymen for example, when using directive antennas. I also briefly discussed how signal jamming can be avoided in one direction by moving the main signal beam away from the jamming source. Hence, it was noted that the use of directive antenna beam patterns held 
several advantages over a omni-directional antennas in multipath or hostile environments. Finally, in Chapter 2, I defined the problem statement and identified the knowledge gap that I aimed to bridge with the research work presented in this thesis.

In Chapter 3, I reviewed the previous work done in the areas of reconfigurable antennas, conformal body wearable antennas and beam steering algorithms. I first reviewed the development of antenna arrays as pattern reconfigurable antennas used for satellite communication. I then looked at how mobile BS antennas used arrays for pattern diversity within cellular cells. It was observed that in the last two decades an increased focus on reconfigurable antennas resulted in the development of single unit pattern reconfigurable antennas for mobile communication devices.

I then looked at the state of the art of pattern reconfigurable antennas. In majority of the single unit pattern reconfigurable antennas studied, the use of $\mathrm{p}-\mathrm{i}-\mathrm{n}$ diodes, RF switches, FET switches and varactor diodes were used to switch the current between different radiating elements of the antenna, thereby creating a switchable pattern in them based on the ON/OFF statuses of the switches used. The use of FSS in antennas were discussed next. FSS consist of a wall consisting of vertical columns of reconfigurable unit cells surrounding a central antenna. The antenna in the middle radiates an omni pattern. But by reconfiguring columns of unit cells, they can be made transparent to or opaque to the EM waves incident on them. Therefore the beam generated by the antenna will be directed in a certain azimuth direction. The columns in the FSS can be reconfigured to steer this beam giving it $360^{\circ}$ coverage.

I next looked at reconfigurable antennas built using HIS. A HIS consists of an array of conducting patches embedded in the antenna substrate. In some of the designs studied, some or all the patches in the HIS were connected to the ground using vias. HIS, although not directly aiding in providing pattern reconfigurability to antennas, help to reduce the antenna profile height (thickness), which assist in making unobtrusive antennas conformal to some platforms. Some other pattern reconfigurable antennas studied consisted of non-planar structures. These antennas although having pattern reconfigurability, were identified as having structural designs that are not ideal for conformal antenna applications.

I then studied some of the previous work done in the development of multi-port pattern reconfigurable antennas with beam steerable capability. The 4-port planar SLA was considered as a good solution for a conformal body-wearable beam steerable antenna as it had a $0<\phi<360^{\circ}$ azimuth beam steering capability. Its switching mechanism using a SP4T RF switch was easy to implement. Its tilted beam pattern consisted of a tilted beam $\left(\theta=43^{\circ}\right)$ covering one spatial quadrant in the azimuth direction. I then briefly studied some of the previously published work on flexible and conformal pattern reconfigurable antennas. Previous work on helmet antennas were also looked at. After studying the literature, it was 


\section{Conclusion}

noted that there was a knowledge gap in the area of development of single unit conformal body wearable pattern reconfigurable antennas for mobile and infantry communication.

Finally, in Chapter 3, I studied ways of implementing a DoA algorithm for the antenna to be built. The different switching schemes such as SC, SSC and SEC previously mentioned in literature were also studied, with a view of implementing a robust beam switching algorithm for the antenna.

In Chapter 4, I designed and built a prototype of a conformal antenna on a hemispherical substrate. I first studied the adaptability of the planar SLA by Mehta and Mirshekar-Syahkal (2007) in making it conformal to doubly curved surfaces.

When a hemispherical substrate's thickness was varied from $11.25 \mathrm{~mm}$ to $33.75 \mathrm{~mm}$, it was observed that the HSLA's directivity varied from $7.5 \mathrm{~dB}$ to $9 \mathrm{~dB}$, with its highest directivity at $20.25 \mathrm{~mm}$ substrate thickness. When the hemispherical shell substrate's radius was varied from $192.4 \mathrm{~mm}$ to $412.3 \mathrm{~mm}$, its directivity varied from $5.43 \mathrm{~dB}$ to $9.4 \mathrm{~dB}$ with the highest directivity occurring at $302.4 \mathrm{~mm}$. However, its best radiation pattern occurred at a radius of $274.9 \mathrm{~mm}$. The third analytical study conducted was on transforming the SLA on a planar circular disc substrate to a conformal SLA on a hemi-prolate substrate shape, while keeping its surface area fixed. It was observed that when the hemi-prolate shape's vertical axis $(\boldsymbol{c})$ to horizontal axis $(\boldsymbol{a})$ ratio $\left(\frac{\boldsymbol{c}}{\boldsymbol{a}}\right)$ was varied, its directivity steadily increased from 8.43 $\mathrm{dB}$ to $9.04 \mathrm{~dB}$ and then it fell sharply for hemi-prolate shapes with $\frac{c}{a}>1$ due to radiation pattern shape deterioration.

Therefore, after studying these results, I designed and fabricated a prototype of a HSLA whose radius was $274.5 \mathrm{~mm}$ and substrate thickness was $22.5 \mathrm{~mm}$, using a Kevlar $\left(\varepsilon_{r}=3.4\right)$ substrate and a foam padding $\left(\varepsilon_{r} \approx 1.0\right)$ underneath as a second substrate. The conformal HSLA prototype built was then used in an experiment to observe how its beam steerable capability could be used for performance enhancement in a noisy EM and multipath environment.

A beam switching algorithm that used a hybrid SC and SEC switching method was used on a mobile Windows®device. The HSLA with a SP4T switch and a switching control circuitry that received switching decision feedback from the mobile device, acted as the BS antenna connected to a router. When the RSSI at the mobile device dropped by $50 \%$ (i.e RSSI drops by $3 \mathrm{~dB}$ ), it sends the signal to the HSLA at the BS to switch to the best port that gives the highest RSSI.

This experimental setup was used in a HD video conferencing call scenario and I was able to demonstrate that with the beam switching algorithm running with the HSLA, I was able to continue with the HD call while moving the mobile device around in a $0<\phi<360^{\circ}$ azimuth range, achieving high bit rate and low packet loss and frame errors. 
In Chapter 5, I conducted further experiments using HSLA as a body wearable pattern reconfigurable antenna in a high throughput HD video call application. The algorithm used in the previous chapter for Windows was modified to suit a mobile hardware platform using a Linux based SBC, connected to the HSLA. The new algorithm running on the SBC, contained not only the hybrid SC and SEC schemes mentioned earlier but also a periodic SC scheme which combined to provide a robust beam switching capability for the HSLA to track the direction of arrival of the BS signal.

In this experiment, the HSLA was placed on a rotating platform (i.e mobile MS scenario) and the BS antenna which used a highly directive Horn antenna was fixed. The SBC acted as a WiFi hotspot to which another device was connected. A HD video call was setup between this device and an external device, and the video call statistics were observed while rotating the HSLA in the azimuth direction. It was observed that compared to the case of not running the beam switching algorithm for the HSLA, when the algorithm was used communication was possible using the HD video conferencing call while maintaining a high throughput. The difference in the available bandwidth in a high noise and multipath environment when using the algorithm was a gain of $7 \mathrm{Mbps}$ in the bandwidth compared to the scenario where no communication was possible (0 Mbps bandwidth and $98 \%$ packet loss) without it, in the same noisy environment.

The experiment conducted thus proved the robust beam steering capability of this software controlled body wearable conformal pattern reconfigurable SLA.

In the penultimate chapter of this thesis, I presented preliminary studies on 3 different antennas at higher frequency bands ( $\mathrm{Ku}$ band, $\mathrm{K}$ band and $\mathrm{Ka}$ band). I first scaled the SLA presented by Mehta and Mirshekar-Syahkal (2007) in order to design a planar SLA with $4.65 \lambda_{g}$ circumference. The preliminary results observed indicated fairly good impedance matching at $12 \mathrm{GHz}$ but patterns with undesirable minor lobes in other directions were observed. Next, I studied a planar SLA for the K band $(25 \mathrm{GHz})$. The SLA with HHIS by Deo et al. (2010) was scaled down from $4.7 \mathrm{GHz}$ to $25 \mathrm{GHz}$ by using the ratio of 5.32 as a scaling factor. The preliminary simulated results for this antenna were very encouraging as it produced a high directive pattern with low minor lobes. It was observed that the generated antenna beam had less beam tilt in the elevation plane than what was observed in the antenna by Deo et al. (2010). The final design I studied was the $30 \mathrm{GHz}$ planar SLA. The simulated results for this antenna indicated unsatisfactory impedance matching at the desired frequency of $30 \mathrm{GHz}$. The generated radiation patterns were also not ideal, with minor lobes in the reverse azimuth direction. 


\section{Conclusion}

Hence, for all 3 antennas studied, more work was necessary to optimise their physical parameters in order to produce the desired tilted beam patterns and impedance matching bandwidth at the respective frequencies.

I then looked at ways of improving the current beam switching algorithm used for the HSLA. It was noted that use of SNR as a switching metric in the SC and SEC combining schemes instead of the currently used RSSI would produce a more accurate BER performance in the presence of jamming or interference signals. In addition, I proposed implementing a DoA algorithm which takes into account the power of all 3 adjacent beam positions in the HSLA, in order to compute the estimator $\boldsymbol{d}$, as explained in Sanchis et al. (2002). Finally, I proposed to study further the implementation of a moving optimal switching threshold for the beam switching algorithm presented in this thesis.

\subsection{Contribution to knowledge}

The findings reported in this thesis make two new important contributions to existing knowledge on beam steerable pattern reconfigurable antennas.

First, a conformal single unit pattern reconfigurable antenna with 4 identical beams which can be switched in the 4 quadrants of the space was designed and built. Even though numerous pattern reconfigurable antennas were reported in literature, many such work involved the use of antenna arrays with phase shifting to perform beam scanning. The single unit pattern reconfigurable antennas reported in literature mostly involved diodes and RF switches on the surface of the antenna element that switched the current to different radiating elements of the antenna. These antennas often created dissimilar radiation Gain patterns in different directions. Some reported work demonstrated generating identical beam patterns in different directions. However, these antennas too suffered from the problem of having diodes or other switching elements placed on the surface of the antenna to control the switching between different radiating elements of the antenna. Although work on 4-beam, switched port SLA have been reported in the past, these involved a finite substrate cut to the shape of the ground. In addition, they were all planar structures.

The work I have presented consists of a conformal single unit pattern reconfigurable antenna (i.e HSLA) which generates 4 identical beam patterns in the 4 spatial quadrants in the space. The beam switching of this antenna is done using a SP4T RF switch which feeds the 4 ports of the conformal SLA. The SP4T switch is electronically controlled using control voltages fed to it using a hardware platform. Therefore, this is the first instance of using such a pattern reconfigurable SLA conformal to an existing platform. 
The second important contribution of the work reported in this thesis is the beam switching algorithm which runs on the hardware platform that controls the SP4T switch. The algorithm used has been developed for Windows and then modified to suit a SBC on a Linux platform. It uses the RSSI as a switching metric and conducts the beam switching between the 4 ports using a hybrid SC and SEC scheme, as explained earlier. The robustness and the usefulness of this algorithm has been demonstrated in a high multipath and high noise EM environment where it was able to sustain a high throughput communication link.

Therefore, the work presented in this theses presents a novel, software controlled conformal beam steerable antenna, which conducts the beam switching to track the signal of interest to maintain high throughput, even in a high noise high multipath environment.

This concludes the main body of this thesis. 



\section{References}

Aboufoul, T., Alomainy, A., and Parini, C. (2012). Reconfiguring uwb monopole antenna for cognitive radio applications using gaas fet switches. IEEE Antennas and Wireless Propagation Letters, 11:392-394.

Aboufoul, T., Parini, C., Chen, X., and Alomainy, A. (2013). Pattern-reconfigurable planar circular ultra-wideband monopole antenna. IEEE Transactions on Antennas and Propagation, 61(10):4973-4980.

Abutarboush, H. F., Nilavalan, R., Cheung, S. W., Nasr, K. M., Peter, T., Budimir, D., and Al-Raweshidy, H. (2012). A reconfigurable wideband and multiband antenna using dual-patch elements for compact wireless devices. IEEE Transactions on Antennas and Propagation, 60(1):36-43.

Alakija, C. and Stapleton, S. P. (1992). A mobile base station phased array antenna. In 1992 IEEE International Conference on Selected Topics in Wireless Communications, pages $118-121$.

Alexandru, R.-C. and Elena-Simona, L. (2015). Comparison of detection techniques for multipath propagation of pseudolite signals used in dense industrial environments. Procedia Engineering, 100:1294 - 1300.

Alexeff, I., Anderson, T., Parameswaran, S., Pradeep, E. P., Hulloli, J., and Hulloli, P. (2006). Experimental and theoretical results with plasma antennas. IEEE Transactions on Plasma Science, 34(2):166-172.

Ali, T. and Biradar, R. C. (2016). Design of three metasurface acting as left-handed material for reconfigurable antenna. In 2016 IEEE International Conference on Computational Intelligence and Computing Research (ICCIC), pages 1-6.

Alley, G. D. (1970). Interdigital capacitors and their application to lumped-element microwave integrated circuits. IEEE Transactions on Microwave Theory and Techniques, 18(12):1028-1033.

Aloman, A., Ispas, A. I., Ciotirnae, P., Sanchez-Iborra, R., and Cano, M. D. (2015). Performance evaluation of video streaming using mpeg dash, rtsp, and rtmp in mobile networks. In 2015 8th IFIP Wireless and Mobile Networking Conference (WMNC), pages 144-151. 
Angelucci, A., Audagnotto, P., Corda, P., Obino, P., Piarulli, F., and Piovano, B. (1994). High performance microstrip networks for multibeam and reconfigurable operation in mobile-radio systems. In 1994 IEEE GLOBECOM. Communications: The Global Bridge, volume 3, pages 1717-1721 vol.3.

Armogida, A. and Peixeiro, C. (1997). Microstrip patch antenna array for a mobile communication system base station. In IEEE Antennas and Propagation Society International Symposium 1997. Digest, volume 1, pages 430-433 vol.1.

Autodesk (2013).3ds max@ONLINE.

Bahng, K., Jung, C. W., and Kim, K. (2009). Design and measurement of beam reconfigurable antenna based on folded dipole structure. Electronics Letters, 45(3):138-139.

Bai, Y. Y., Xiao, S., Tang, M. C., Ding, Z. F., and Wang, B. Z. (2011). Wide-angle scanning phased array with pattern reconfigurable elements. IEEE Transactions on Antennas and Propagation, 59(11):4071-4076.

Barro, O. A., Himdi, M., and Lafond, O. (2017). Performances of monopole plasma antenna. In 2017 11th European Conference on Antennas and Propagation (EUCAP), pages 35683571 .

Beaulieu, N. C. (2008). Switching rates of dual selection diversity and dual switch-and-stay diversity. IEEE Transactions on Communications, 56(9):1409-1413.

Bernhard, J. T. (2007). Reconfigurable Antennas, volume 4 of Synthesis Lectures on Antennas, pages 1-66. Morgan and Claypool.

Besoli, A. G. and Flaviis, F. D. (2011). A multifunctional reconfigurable pixeled antenna using mems technology on printed circuit board. IEEE Transactions on Antennas and Propagation, 59(12):4413-4424.

Boisbouvier, N., Bolzer, F. L., and Louzir, A. (2002). A compact radiation pattern diversity antenna for wlan applications. In IEEE Antennas and Propagation Society International Symposium (IEEE Cat. No.02CH37313), volume 4, pages 64-67 vol.4.

Cai, X.-T., guo Wang, A., Ma, N., and Leng, W. (2012). Novel radiation pattern reconfigurable antenna with six beam choices. The Journal of China Universities of Posts and Telecommunications, 19(2):123 - 128.

Cao, W., Zhang, B., Liu, A., Yu, T., Guo, D., and Pan, K. (2012). A reconfigurable microstrip antenna with radiation pattern selectivity and polarization diversity. IEEE Antennas and Wireless Propagation Letters, 11:453-456.

Chang, B. C. C., Qian, Y., and Itoh, T. (1999). A reconfigurable leaky mode/patch antenna controlled by pin diode switches. In IEEE Antennas and Propagation Society International Symposium. 1999 Digest. Held in conjunction with: USNC/URSI National Radio Science Meeting (Cat. No.99CH37010), volume 4, pages 2694-2697 vol.4. 
Chau, Y. A. and Chen, Y. H. (2010). Multibranch switch-and-stay diversity with a new switch statistic on wireless fading channels. In TENCON 2010 - 2010 IEEE Region 10 Conference, pages 2367-2371.

Chen, B., Leng, W., Wang, A. G., and Zhao, G. H. (2012). Compact ultra-wideband antenna with reconfigurable notched bands. Electronics Letters, 48(19):1175-1176.

Chen, D., Liu, Z., Wang, L., Dou, M., Chen, J., and Li, H. (2013). Natural disaster monitoring with wireless sensor networks: A case study of data-intensive applications upon low-cost scalable systems. Mob. Netw. Appl., 18(5):651-663.

Choudhury, R. R., Yang, X., Ramanathan, R., and Vaidya, N. H. (2006). On designing mac protocols for wireless networks using directional antennas. IEEE Transactions on Mobile Computing, 5(5):477-491.

Cisco (2006). Cisco aironet 1200 series access point data sheet@ONLINE.

Clarricoats, P. J. B., Hai, Z., Brown, R. C., poulton, G. T., and Crone, G. A. E. (1988). A reconfigurable satellite reflector antenna. In 1988 18th European Microwave Conference, pages $482-487$.

Comp, C. J. and Axelrad, P. (1998). Adaptive snr-based carrier phase multipath mitigation technique. IEEE Transactions on Aerospace and Electronic Systems, 34(1):264-276.

Coomans, W., Moraes, R. B., Hooghe, K., Duque, A., Galaro, J., Timmers, M., van Wijngaarden, A. J., Guenach, M., and Maes, J. (2015). Xg-fast: the 5th generation broadband. IEEE Communications Magazine, 53(12):83-88.

Cooper, H. and McComas, H. (1960). Synthesis of csc2 type antenna patterns using twodimensional surface wave arrays. In 1958 IRE International Convention Record, volume 8, pages $24-34$.

CST (2013). Cst microwave studio@ONLINE.

Cupo, R. L., Golden, G. D., Martin, C. C., Sherman, K. L., Sollenberger, N. R., Winters, J. H., and Wolniansky, P. W. (1997). A four-element adaptive antenna array for is-136 pcs base stations. In 1997 IEEE 47th Vehicular Technology Conference. Technology in Motion, volume 3, pages $1577-1581$ vol.3.

Dagang, A. N., Ramli, N. F., Alias, N. S., and Ali, M. T. (2016a). Effect of plasma parameters on monopole plasma antenna performance. In 2016 International Conference on Computer and Communication Engineering (ICCCE), pages 434-438.

Dagang, A. N., Ramli, N. F., Alias, N. S., and Ali, M. T. (2016b). Effect of plasma parameters on monopole plasma antenna performance. In 2016 International Conference on Computer and Communication Engineering (ICCCE), pages 434-438. 
Daly, M. P. and Bernhard, J. T. (2010). Beamsteering in pattern reconfigurable arrays using directional modulation. IEEE Transactions on Antennas and Propagation, 58(7):22592265 .

Damis, H. A., Mirzavand, R., Chung, H. J., and Mousavi, P. (2016). Flexible printed square loop antennas for wearable applications. In 2016 17th International Symposium on Antenna Technology and Applied Electromagnetics (ANTEM), pages 1-2.

Deo, P., Mehta, A., Mirshekar-Syahkal, D., Massey, P. J., and Nakano, H. (2009a). A beam switched loop antenna for pattern reconfigurable applications. In 2009 Loughborough Antennas Propagation Conference, pages 157-160.

Deo, P., Mehta, A., Mirshekar-Syahkal, D., Massey, P. J., and Nakano, H. (2010). Thickness reduction and performance enhancement of steerable square loop antenna using hybrid high impedance surface. IEEE Transactions on Antennas and Propagation, 58(5):1477-1485.

Deo, P., Mehta, A., Mirshekar-Syahkal, D., and Nakano, H. (2009b). An his-based spiral antenna for pattern reconfigurable applications. IEEE Antennas and Wireless Propagation Letters, 8:196-199.

Deo, P., Mirshekar-Syahkal, D., Pant, M., Mehta, A., and Nakano, H. (2011a). Single pole four throw (sp4t) switch integration with a steerable square loop antenna. In 2011 IEEE International Symposium on Antennas and Propagation (APSURSI), pages 2043-2046.

Deo, P., Pant, M., Mehta, A., Mirshekar-Syahkal, D., and Nakano, H. (2011b). Implementation and simulation of commercial rf switch integration with steerable square loop antenna. Electronics Letters, 47(12):686-687.

Devices, A. (2009). Hmc241lp3/241lp3e gaas mmic sp4t non-reflective switch, dc - 4 ghz.

Ding, T. N., Sillard, P., and Ho, P. T. (1995). A simple reconfigurable antenna. In IEEE/LEOS 1995 Digest of the LEOS Summer Topical Meetings. Flat Panel Display Technology, pages 63-64.

Dobias, F. and Gunther, J. (1995). Reconfigurable array antennas with phase-only control of quantized phase shifters. In 1995 IEEE 45th Vehicular Technology Conference. Countdown to the Wireless Twenty-First Century, volume 1, pages 35-39 vol.1.

Dragūnas, K. and Borre, K. (2010). Indoor multipath mitigation. In 2010 Ubiquitous Positioning Indoor Navigation and Location Based Service, pages 1-8.

Duraisamy, P., Jang, W. M., Nguyen, L., and Steiner, A. (2014). Multipath error mitigation using self-encoded spread spectrum for binary-offset-carrier signals. IET Communications, 8(7):1048-1055.

Ebine, Y. and Ito, M. (1997). Design of a dual-beam antenna used for base station of cellular mobile radios. Electronics and Communications in Japan (Part I: Communications), 80(12):38-46. 
Edalati, A. and Denidni, T. A. (2011). High-gain reconfigurable sectoral antenna using an active cylindrical fss structure. IEEE Transactions on Antennas and Propagation, 59(7):2464-2472.

Edalati, A. and Denidni, T. A. (2013). Frequency selective surfaces for beam-switching applications. IEEE Transactions on Antennas and Propagation, 61(1):195-200.

Engelbrecht, J. M., Weber, R., and Michler, O. (2016). Reduction of multipath propagation influences at poa positioning using uniform circular array antennas analyses based on measurements in vehicular scenarios. In 2016 13th Workshop on Positioning, Navigation and Communications (WPNC), pages 1-5.

Feresidis, A. P., Vardaxoglou, J. C., and Palikaras, G. K. (2011). Advances in low-profile fabry-perot cavity base station antennas using passive periodic surfaces. In 2011 IEEE-APS Topical Conference on Antennas and Propagation in Wireless Communications, pages 894-897.

Fetterolf, P. C. and Price, K. M. (1985). A computer-controlled adaptive antenna system. In MILCOM 1985 - IEEE Military Communications Conference, volume 2, pages 530-533.

Fielitz, H. and ter Haseborg, J. L. (2014). Reliable wi-fi communication in emc critical multipath propagation environment using phased array antennas. In 2014 IEEE International Symposium on Electromagnetic Compatibility (EMC), pages 588-593.

Fortinberry, J., Hood, Z., and Topsakal, E. (2005). A helmet mounted gps antenna. In 2005 IEEE Antennas and Propagation Society International Symposium, volume 3A, pages 471-474 vol. 3 A.

Foundation, R. P. (2015). Raspberry pi b@ONLINE.

Freeman, J. L., Lamberty, B. J., and Andrews, G. S. (1992). Optoelectronically reconfigurable monopole antenna. Electronics Letters, 28(16):1502-1503.

Gafarov, E. R., Stankovsky, A. V., and Salomatov, Y. P. (2016). A gnss quadrupole antenna with a spatial polarizer for the suppression of low-angle multipath. In 2016 International Siberian Conference on Control and Communications (SIBCON), pages 1-4.

Geier, J. (2015). Designing and Deploying 802.11 Wireless Networks: A Practical Guide to Implementing 802.1 In and 802.1 1 ac Wireless Networks For Enterprise-Based Applications (Networking Technology). Cisco Press.

Geomagic (2013). Geomagic studio@ ONLINE.

Ghaderi, M., Moradi, G., and Mousavi, P. (2017). Numerical study on a wideband plasma folded-dipole antenna. IEEE Antennas and Wireless Propagation Letters, 16:1253-1256.

Ghose, R. (1964). Electronically adaptive antenna systems. IEEE Transactions on Antennas and Propagation, 12(2):161-169. 
Giri, S. and Wall, R. W. (2008). A safety critical network for distributed smart traffic signals. IEEE Instrumentation Measurement Magazine, 11(6):10-16.

GNU Radio Website (accessed May 2016).

Gong, L., Chan, K. Y., and Ramer, R. (2013). A reconfigurable spiral antenna with wide beam coverage. In 2013 IEEE Antennas and Propagation Society International Symposium (APSURSI), pages 206-207.

Ha, S. J. and Jung, C. W. (2011). Reconfigurable beam steering using a microstrip patch antenna with a u-slot for wearable fabric applications. IEEE Antennas and Wireless Propagation Letters, 10:1228-1231.

Halili, N. A., Ali, M. T., Zali, H. M., Ja' afar, H., Pasya, I., and Dagang, A. N. (2013). Effects of coupling sleeve designs on an rf charged plasma monopole antenna. In 2013 IEEE International RF and Microwave Conference (RFM), pages 467-470.

Han, T. Y. and Huang, C. T. (2010). Reconfigurable monopolar patch antenna. Electronics Letters, 46(3):199-200.

Hargrave, F. (2001). Hargrave's communications dictionary. IEEE Press, New York.

Haupt, R. L. (2010). Antenna Array Basics, pages 1-44. Wiley-IEEE Press.

Herath, P., Gunawardana, U., and Liyanapathirana, R. (2012). Distributed switch-andexamine combining with threshold-based relaying. In 2012 Australian Communications Theory Workshop (AusCTW), pages 13-18.

Herold, D., Griffiths, L., and Fung, T. Y. (2007). Lightweight, high-bandwidth conformal antenna system for ballistic helmets. In MILCOM 2007 - IEEE Military Communications Conference, pages 1-6.

Hirokawa, J., Kaneta, K., Ardo, M., and Goto, N. (1992). An array antenna of slotted cylinder for land mobile base station. In IEEE Antennas and Propagation Society International Symposium 1992 Digest, pages 1061-1064 vol.2.

Home, S. (2016). Smarthome-home automation systems products kits hubs and ideas@ONLINE.

Huang, Y., Wu, L. S., Tang, M., and Mao, J. (2012). Design of a beam reconfigurable thz antenna with graphene-based switchable high-impedance surface. IEEE Transactions on Nanotechnology, 11(4):836-842.

Huff, G. H., Feng, J., Zhang, S., and Bernhard, J. T. (2003). A novel radiation pattern and frequency reconfigurable single turn square spiral microstrip antenna. IEEE Microwave and Wireless Components Letters, 13(2):57-59.

IEEE (1999). Ieee standard for message sets for vehicle/roadside communications. IEEE Std 1455-1999, pages 90-91. 
IEEE (2014). Ieee standard for definitions of terms for antennas. IEEE Std 145-2013 (Revision of IEEE Std 145-1993), pages 1-50.

Jaafar, H., Omar, S., Shafie, R., Abdullah, R., Ismail, N., and Dagang, A. N. (2017). Simulation study of monopole plasma antenna for $2.4 \mathrm{ghz}$ application. In TENCON 2017 2017 IEEE Region 10 Conference, pages 2927-2930.

Jais, M. I., Jamlos, M. F., Jusoh, M., Sabapathy, T., and Kamarudin, M. R. (2013). 2.45 ghz beam-steering textile antenna for wban application. In 2013 IEEE Antennas and Propagation Society International Symposium (APSURSI), pages 200-201.

Jataprolu, M. K., Michalopoulos, D. S., and Schober, R. (2011). Optimal switch and stay combining (ssc) under switching rate constraints. In 2011 IEEE Wireless Communications and Networking Conference, pages 1676-1681.

Javed, A., Bhellar, B., and Tahir, F. A. (2015). Reconfigurable body worn antenna for bluetooth and wimax. In 2015 12th International Bhurban Conference on Applied Sciences and Technology (IBCAST), pages 571-573.

Jazi, M. N. and Denidni, T. A. (2010a). Agile radiation-pattern antenna based on active cylindrical frequency selective surfaces. IEEE Antennas and Wireless Propagation Letters, 9:387-388.

Jazi, M. N. and Denidni, T. A. (2010b). Frequency selective surfaces and their applications for nimble-radiation pattern antennas. IEEE Transactions on Antennas and Propagation, 58(7):2227-2237.

Jiang, H., Patterson, M., Brown, D., Zhang, C., Pan, K., Subramanyam, G., Kuhl, D., Leedy, K., and Cerny, C. (2012). Miniaturized and reconfigurable cpw square-ring slot antenna loaded with ferroelectric bst thin film varactors. IEEE Transactions on Antennas and Propagation, 60(7):3111-3119.

Jnana, N. J. and Sheeba, O. (2015). Performance analysis of polarization reconfigurable antenna using metasurface. In 2015 International Conference on Control Communication Computing India (ICCC), pages 418-423.

Johannisson, B. (1998). Adaptive base station antennas for mobile communication systems. In 1998 IEEE-APS Conference on Antennas and Propagation for Wireless Communications (Cat. No.98EX184), pages 49-52.

Jung, C. W., Lee, M.-J., Li, G. P., and Flaviis, F. D. (2006). Reconfigurable scan-beam singlearm spiral antenna integrated with rf-mems switches. IEEE Transactions on Antennas and Propagation, 54(2):455-463.

Kang, S. and Jung, C. W. (2015). Wearable fabric reconfigurable beam steering antenna for on/off-body communication system. In 2015 IEEE International Symposium on Antennas and Propagation USNC/URSI National Radio Science Meeting, pages 1211-1212. 
Kang, W., Lee, S., and Kim, K. (2010). Design of symmetric beam pattern reconfigurable antenna. Electronics Letters, 46(23):1536-1537.

Khosravi, F., Moghadas, H., and Mousavi, P. (2015). A gnss antenna with a polarization selective surface for the mitigation of low-angle multipath interference. IEEE Transactions on Antennas and Propagation, 63(12):5287-5295.

Kim, I. and Rahmat-Samii, Y. (2011). Rf mems switchable slot patch antenna integrated with bias network. IEEE Transactions on Antennas and Propagation, 59(12):4811-4815.

Kim, J. Y., Jung, C. W., and Lee, B. (2011a). Reconfigurable beam steering antenna using double loops. In 2011 IEEE International Symposium on Antennas and Propagation (APSURSI), pages 1570-1572.

Kim, J. Y., Lee, B., and Jung, C. W. (2011b). Reconfigurable beam-steering antenna using double loops. Electronics Letters, 47(7):430-431.

Ko, C. H., Tarn, I. Y., and Chung, S. J. (2013). A compact dual-band pattern diversity antenna by dual-band reconfigurable frequency-selective reflectors with a minimum number of switches. IEEE Transactions on Antennas and Propagation, 61(2):646-654.

Lai, M. I., Wu, T. Y., Hsieh, J. C., Wang, C. H., and Jeng, S. K. (2009). Design of reconfigurable antennas based on an 1-shaped slot and pin diodes for compact wireless devices. IET Microwaves, Antennas Propagation, 3(1):47-54.

Lebaric, J. and Tan, A.-T. (2000a). Ultra-wideband conformal helmet antenna. In 2000 Asia-Pacific Microwave Conference. Proceedings (Cat. No.00TH8522), pages 1477-1481.

Lebaric, J. and Tan, A.-T. (2000b). Ultra-wideband rf helmet antenna. In MILCOM 2000 Proceedings. 21st Century Military Communications. Architectures and Technologies for Information Superiority (Cat. No.00CH37155), volume 1, pages 591-594 vol.2.

Lee, C., Kim, Y., and Jung, C. W. (2013). 3-d beam steering antenna for beam - reconfigurable system. In 2013 IEEE Antennas and Propagation Society International Symposium (APSURSI), pages 1438-1439.

Lee, K. W., Rojas, R. G., and Surittikul, N. (2006). A pattern-reconfigurable microstrip antenna element. Microwave and Optical Technology Letters, 48(6):1117-1119.

Li, M., Xiao, S. Q., and Wang, B. Z. (2013). Pattern-reconfigurable antenna for on-body communication. In 2013 IEEE MTT-S International Microwave Workshop Series on RF and Wireless Technologies for Biomedical and Healthcare Applications (IMWS-BIO), pages $1-3$.

Li, Y., Zhang, Z., Chen, W., Feng, Z., and Iskander, M. F. (2009). A quadband antenna with reconfigurable feedings. IEEE Antennas and Wireless Propagation Letters, 8:1069-1071. 
Li, Y., Zhang, Z., Zheng, J., and Feng, Z. (2011a). Compact heptaband reconfigurable loop antenna for mobile handset. IEEE Antennas and Wireless Propagation Letters, 10:1162-1165.

Li, Y., Zhang, Z., Zheng, J., Feng, Z., and Iskander, M. F. (2011b). Experimental analysis of a wideband pattern diversity antenna with compact reconfigurable cpw-to-slotline transition feed. IEEE Transactions on Antennas and Propagation, 59(11):4222-4228.

Libtins Library (accessed Dec 2015).

Lim, I. and Lim, S. (2013). Monopole-like and boresight pattern reconfigurable antenna. IEEE Transactions on Antennas and Propagation, 61(12):5854-5859.

Lim, S. and Ling, H. (2007). Design of electrically small, pattern reconfigurable yagi antenna. Electronics Letters, 43(24):1326-1327.

Linden, D. S. (2001). In-situ evolution of a reconfigurable antenna. In 2001 IEEE Aerospace Conference Proceedings (Cat. No.01TH8542), volume 5, pages 2333-2338 vol.5.

Liu, J. (2016). Wireless multipath fading channels modeling and simulation based on sumof-sinusoids. In 2016 First IEEE International Conference on Computer Communication and the Internet (ICCCI), pages 165-168.

Liu, X., Sheth, A., Kaminsky, M., Papagiannaki, K., Seshan, S., and Steenkiste, P. (2009). Dirc: Increasing indoor wireless capacity using directional antennas. SIGCOMM Comput. Commun. Rev., 39(4):171-182.

Mansutti, G., Melazzi, D., and Capobianco, A. D. (2016). Hybrid metal-plasma yagi-uda antenna for microwave applications. In 2016 46th European Microwave Conference (EuMC), pages 727-730.

Marais, J., Duflos, E., Viandier, N., Nahimana, D. F., and Rabaoui, A. (2010). Advanced signal processing techniques for multipath mitigation in land transportation environment. In 13th International IEEE Conference on Intelligent Transportation Systems, pages 1480-1485.

Mehta, A. and Mirshekar-Syahkal, D. (2007). Pattern steerable square loop antenna. Electronics Letters, 43(9):491-493.

Mehta, A., Mirshekar-Syahkal, D., and Nakano, H. (2006). Beam adaptive single arm rectangular spiral antenna with switches. IEE Proceedings - Microwaves, Antennas and Propagation, 153(1):13-18.

Melazzi, D., Carlo, P. D., Trezzolani, F., Lancellotti, V., Manente, M., Pavarin, D., Rigobello, F., and Capobianco, A. D. (2017). First experimental characterization of a gaseous plasma antenna in the uhf band. In 2017 11th European Conference on Antennas and Propagation (EUCAP), pages 3213-3217. 
Mikenas, V., Rassweiler, G., Lehman, D., Bustelo, R., Payne, L., and Diemond, G. (1975). An adaptive null-steering phased array. In 1975 Antennas and Propagation Society International Symposium, volume 13, pages 315-318.

Milligan, T. A. (2005). Modern Antenna Design, chapter 1, pages 1-41. Wiley-IEEE Press.

Moradi, H., Refai, H. H., and Lopresti, P. G. (2012). Switch-and-stay and switch-and-examine dual diversity for high-speed free-space optics links. IET Optoelectronics, 6(1):34-42.

MVG (2014). Satimo starlab@ONLINE.

Nair, S. V. S. and Ammann, M. J. (2010). Reconfigurable antenna with elevation and azimuth beam switching. IEEE Antennas and Wireless Propagation Letters, 9:367-370.

Navda, V., Subramanian, A. P., Dhanasekaran, K., Timm-Giel, A., and Das, S. (2007). Mobisteer: Using steerable beam directional antenna for vehicular network access. In ACM MobiSys (Best Paper Award), Puerto Rico. Association for Computing Machinery, Inc.

Nicopolitidis, P., Obaidat, M., and Papadimitriou, G. I. (2003). Wireless Networks. John Wiley \& Sons.

Niroo-Jazi, M. and Denidni, T. A. (2013). Electronically sweeping-beam antenna using a new cylindrical frequency-selective surface. IEEE Transactions on Antennas and Propagation, 61(2):666-676.

Ogawa, Y., Ohmiya, M., and Itoh, K. (1983). An analog open-loop adaptive-array antenna system. IEEE Transactions on Aerospace and Electronic Systems, AES-19(1):89-102.

Ong, L. T. and Tan, P. K. (2015). Multipath mitigation techniques based on spatial smoothing linear antenna arrays. In 2015 IEEE-APS Topical Conference on Antennas and Propagation in Wireless Communications (APWC), pages 246-249.

Pal, A., Mehta, A., Lewis, R., and Clow, N. (2015). Reconfigurable phased array antenna enabling a high gain wide angle beam scanning. In 2015 IEEE International Symposium on Antennas and Propagation USNC/URSI National Radio Science Meeting, pages 22072208.

Pal, A., Mehta, A., and Marhic, M. E. (2013a). Generating a pure circularly polarised axial beam from a pattern reconfigurable square loop antenna. IET Microwaves, Antennas Propagation, 7(3):208-213.

Pal, A., Mehta, A., and Mirshekar-Syahkal, D. (2014a). Dual band low-profile switched beam square loop antenna with capacitively coupled feeds. In The 8th European Conference on Antennas and Propagation (EuCAP 2014), pages 2578-2582.

Pal, A., Mehta, A., Mirshekar-Syahkal, D., Deo, P., and Nakano, H. (2014b). Dual-band low-profile capacitively coupled beam-steerable square-loop antenna. IEEE Transactions on Antennas and Propagation, 62(3):1204-1211. 
Pal, A., Mehta, A., Mirshekar-Syahkal, D., Deo, P., and Nakano, H. (2014c). Dual-band low-profile capacitively coupled beam-steerable square-loop antenna. IEEE Transactions on Antennas and Propagation, 62(3):1204-1211.

Pal, A., Mehta, A., Mirshekar-Syahkal, D., and Massey, P. J. (2008). Short-circuited feed terminations on beam steering square loop antennas. Electronics Letters, 44(24):13891390 .

Pal, A., Mehta, A., Mirshekar-Syahkal, D., and Massey, P. J. (2009). Doughnut and tilted beam generation using a single printed star antenna. IEEE Transactions on Antennas and Propagation, 57(10):3413-3418.

Pal, A., Mehta, A., Mirshekar-Syahkal, D., and Nakano, H. (2011). A square-loop antenna with 4-port feeding network generating semi-doughnut pattern for vehicular and wireless applications. IEEE Antennas and Wireless Propagation Letters, 10:338-341.

Pal, A., Mehta, A., Mirshekar-Syahkal, D., and Nakano, H. (2012a). Low-profile capacitively fed steerable square loop antenna. In Proceedings of the 2012 IEEE International Symposium on Antennas and Propagation, pages 1-2.

Pal, A., Mehta, A., Mirshekar-Syahkal, D., and Nakano, H. (2012b). Low-profile steerable loop antenna with capacitively coupled feeds. IEEE Antennas and Wireless Propagation Letters, 11:873-876.

Pal, A., Mehta, A., Mirshekar-Syahkal, D., and Nakano, H. (2013b). A low-profile switchedbeam dual-band capacitively coupled square loop antenna. In 2013 Loughborough Antennas Propagation Conference (LAPC), pages 563-566.

Pal, A., Mehta, A., Mirshekar-Syahkal, D., and Nakano, H. (2017). 2times2 phased array consisting of square loop antennas for high gain wide angle scanning with low grating lobes. IEEE Transactions on Antennas and Propagation, 65(2):576-583.

Palikaras, G. K., Feresidis, A. P., and Parini, C. G. (2011). Advances in conformal metamaterial antennas using high impedance (his) and electromagnetic bandgap (ebg) surfaces. In Proceedings of the 5th European Conference on Antennas and Propagation (EUCAP), pages 3466-3469.

Park, J.-Y., Ryu, H.-K., and Woo, J.-M. (2007). Helmet installed antenna using a halfwavelength circular loop antenna. In 2007 IEEE Antennas and Propagation Society International Symposium, pages 4176-4179.

Patron, D., Piazza, D., and Dandekar, K. R. (2013). Wideband planar antenna with reconfigurable omnidirectional and directional radiation patterns. Electronics Letters, 49(8):516518.

Qian, Y., Chang, B. C. C., Chang, M. F., and Itoh, T. (1999). Reconfigurable leakymode/multifunction patch antenna structure. Electronics Letters, 35(2):104-105. 
Qin, P. Y., Guo, Y. J., Weily, A. R., and Liang, C. H. (2012). A pattern reconfigurable u-slot antenna and its applications in mimo systems. IEEE Transactions on Antennas and Propagation, 60(2):516-528.

Rlinami, M., Morikawa, H., and Aoyama, T. (2000). An adaptive multipath mitigation technique for gps signal reception. In VTC2000-Spring. 2000 IEEE 51st Vehicular Technology Conference Proceedings (Cat. No.00CH37026), volume 2, pages 1625-1629 vol.2.

Rodrigo, D. and Jofre, L. (2012). Frequency and radiation pattern reconfigurability of a multi-size pixel antenna. IEEE Transactions on Antennas and Propagation, 60(5):22192225 .

Rosloniec, S. (1994). Design of stepped transmission line matching circuits by optimization methods. IEEE Transactions on Microwave Theory and Techniques, 42(12):2255-2260.

Sanchis, P., Martinez, J. M., Herrera, J., Polo, V., Corral, J. L., and Marti, J. (2002). A novel simultaneous tracking and direction of arrival estimation algorithm for beam-switched base station antennas in millimeter-wave wireless broadband access networks. In IEEE Antennas and Propagation Society International Symposium (IEEE Cat. No.02CH37313), volume 1, pages 594-597 vol.1.

Sanford, G. (1978). Conformal microstrip phased array for aircraft tests with ats-6. IEEE Transactions on Antennas and Propagation, 26(5):642-646.

Sarrazin, J., Mahe, Y., Avrillon, S., and Toutain, S. (2009). Pattern reconfigurable cubic antenna. IEEE Transactions on Antennas and Propagation, 57(2):310-317.

Sevgi, L. (2003). Antenna Design and Array Processing, pages 127-178. Wiley-IEEE Press.

Shannon, C. E. (1949). Communication in the presence of noise. Proceedings of the IRE, 37(1):10-21.

Shi, Z., Zheng, R., Ding, J., and Guo, C. (2012). A novel pattern-reconfigurable antenna using switched printed elements. IEEE Antennas and Wireless Propagation Letters, 11:1100-1103.

Sievenpiper, D., Zhang, L., Broas, R. F. J., Alexopolous, N. G., and Yablonovitch, E. (1999). High-impedance electromagnetic surfaces with a forbidden frequency band. IEEE Transactions on Microwave Theory and Techniques, 47(11):2059-2074.

Solidworks (2013). Solidworks 3d cad@ ONLINE.

Song, H., Lee, S. H., Lee, S., and Lee, H. S. (2009). 6lowpan-based tactical wireless sensor network architecture for remote large-scale random deployment scenarios. In MILCOM 2009 - 2009 IEEE Military Communications Conference, pages 1-7.

Sun, J., Sun, H., Xu, Y., and Huo, W. (2016). Study on plasma reconfigurable antenna array prototype. In 2016 IEEE International Conference on Ubiquitous Wireless Broadband (ICUWB), pages $1-4$. 
Swaminathan, R., Karagiannidis, G. K., and Roy, R. (2016). Joint antenna and relay selection strategies for decode-and-forward relay networks. IEEE Transactions on Vehicular Technology, 65(11):9041-9056.

Toole, R. and Fok, M. P. (2016). A photonic rf jamming avoidance response system bio-inspired by eigenmannia. In 2016 Optical Fiber Communications Conference and Exhibition (OFC), pages 1-3.

TV, M. (2016). Mobitv@ONLINE.

U1, T. (2004). 300 mbps utral-fast wireless usb adapter@ONLINE.

Valizade, A., Ghobadi, C., Nourinia, J., and Ojaroudi, M. (2012). A novel design of reconfigurable slot antenna with switchable band notch and multiresonance functions for uwb applications. IEEE Antennas and Wireless Propagation Letters, 11:1166-1169.

VNI, C. M. (2017). Cisco visual networking index: Global mobile data traffic forecast update, 2016-2021.

Wahid, P. F., Ali, M. A., and DeLoach, B. C. (2003). A reconfigurable yagi antenna for wireless communications. Microwave and Optical Technology Letters, 38(2):140-141.

Wang, C. X., Haider, F., Gao, X., You, X. H., Yang, Y., Yuan, D., Aggoune, H. M., Haas, H., Fletcher, S., and Hepsaydir, E. (2014). Cellular architecture and key technologies for $5 \mathrm{~g}$ wireless communication networks. IEEE Communications Magazine, 52(2):122-130.

Wang, J. J. H. (2006). Broadband omnidirectional helmet antennas. In 2006 IEEE Antennas and Propagation Society International Symposium, pages 2129-2132.

Wang, J. J. H. and Tillery, J. K. (2000). Broadband miniaturised slow-wave antenna.

Wang, J. J. H. and Triplett, D. J. (2007). Multioctave broadband body-wearable helmet and vest antennas. In 2007 IEEE Antennas and Propagation Society International Symposium, pages $4172-4175$.

Wang, J. J. H. and Tripp, V. K. (1991). Design of multioctave spiral-mode microstrip antennas. IEEE Transactions on Antennas and Propagation, 39(3):332-335.

Wang, L., Jun, O. Y., and Yang, F. (2012). Reconfigurable sectoral antenna using an cylindrical frequency selective surface. In Proceedings of the 2012 IEEE International Symposium on Antennas and Propagation, pages 1-2.

Wu, J., Chang, C., Chin, T., Huang, S., and Chang, S. (2010a). Sidelobe level reduction in wide-angle scanning array system using pattern-reconfigurable antennas. In 2010 IEEE MTT-S International Microwave Symposium, pages 1-1.

Wu, J. C., Chang, C. C., Chin, T. Y., Huang, S. Y., and Chang, S. F. (2010b). Sidelobe level reduction in wide-angle scanning array system using pattern-reconfigurable antennas. In 2010 IEEE MTT-S International Microwave Symposium, pages 1274-1277. 
Wu, Z., Liu, H., and Li, L. (2016). Polarization reconfigurable metasurface superstrate antenna with low profile. In 2016 10th European Conference on Antennas and Propagation $(E u C A P)$, pages $1-2$.

Xiao, S., Wang, B.-Z., and Yang, X.-S. (2003). A novel frequency-reconfigurable patch antenna. Microwave and Optical Technology Letters, 36(4):295-297.

Yaacoub, E., Dawy, Z., and Abu-Dayya, A. (2012). On real-time video streaming over lte networks with mobile-to-mobile cooperation. In 2012 19th International Conference on Telecommunications (ICT), pages 1-6.

Yang, F. and Rahmat-Samii, Y. (2002). A reconfigurable patch antenna using switchable slots for circular polarization diversity. IEEE Microwave and Wireless Components Letters, 12(3):96-98.

Yang, P., Yan, F., Zhou, L., Gao, M., and Yang, F. (2016). A small low-multipath gnss antenna using annular slot loaded ground plane. In 2016 IEEE 5th Asia-Pacific Conference on Antennas and Propagation (APCAP), pages 349-350.

Yuan, J., Liu, S., Kong, X., and Yang, H. (2013). A reconfigurable frequency selective surface for tuning multi-band frequency response separately. In 2013 Proceedings of the International Symposium on Antennas Propagation, volume 02, pages 1288-1290.

Zali, H. M., Ali, M. T., Pasya, I., Halili, N. A., Ja'afar, H., and Hilmi, M. (2013). Design of a cylindrical parabolic reflector on monopole plasma antenna. In 2013 IEEE International $R F$ and Microwave Conference (RFM), pages 344-348.

Zhang, G. M., Hong, J. S., Song, G., and Wang, B. Z. (2012). Design and analysis of a compact wideband pattern-reconfigurable antenna with alternate reflector and radiator. IET Microwaves, Antennas Propagation, 6(15):1629-1635.

Zhang, L., Wu, Q., and Denidni, T. A. (2013). Electronically radiation pattern steerable antennas using active frequency selective surfaces. IEEE Transactions on Antennas and Propagation, 61(12):6000-6007.

Zhang, Y., meng Yu, X., yu Kong, L., peng Zhang, L., and lei Qin, H. (2016). Anti-multipath dual-band gnss antenna design with peano fractal ebg structure. In 2016 Asia-Pacific International Symposium on Electromagnetic Compatibility (APEMC), volume 01, pages $523-525$.

Zhao, J., Fan, P., Beaulieu, N. C., and Lei, X. (2015). Switching rates of selection diversity and switch-and-stay diversity on mixed high-speed train channels. In 2015 International Workshop on High Mobility Wireless Communications (HMWC), pages 51-55.

Zhu, H. L., Cheung, S. W., Liu, X. H., Cao, Y. F., and Yuk, T. I. (2014a). Frequency reconfigurable slot antenna using metasurface. In The 8th European Conference on Antennas and Propagation (EuCAP 2014), pages 2575-2577. 
Zhu, H. L., Cheung, S. W., Liu, X. H., and Yuk, T. I. (2014b). Design of polarization reconfigurable antenna using metasurface. IEEE Transactions on Antennas and Propagation, 62(6):2891-2898.

Zohur, A., Mopidevi, H., Rodrigo, D., Unlu, M., Jofre, L., and Cetiner, B. A. (2013). Rf mems reconfigurable two-band antenna. IEEE Antennas and Wireless Propagation Letters, $12: 72-75$. 



\section{Appendix A}

\section{C code use for beam steering algorithm}

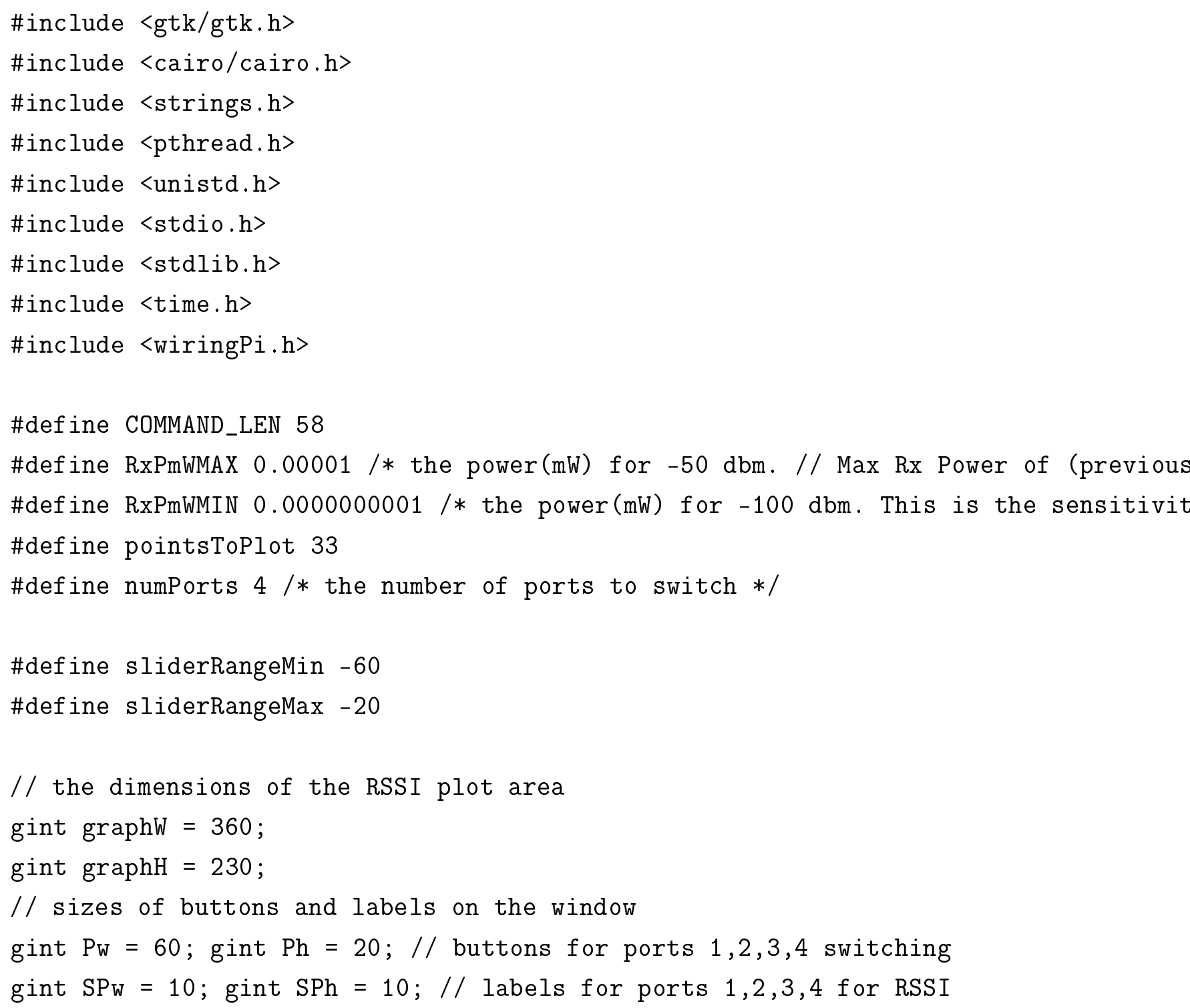


double rssiNew, rssiold; /* the RSSI value to be updated by the thread and the refere GtkTextBuffer *rssi, *refSVal; /* the text buffer to display the current RSSI value a GtkTextBuffer *P1RSSI, *P2RSSI, *P3RSSI, *P4RSSI; // the text buffers to display RSSI GtkTextBuffer *Port; // the text buffer to display the selected port

gboolean rssiStop, switchNow, manualon; // boolean flags to stop threads gboolean startTimer; // boolean value to start rescan rounds when RSSI < $-74 \mathrm{dBm}$ static guint timer1, timer2, timer3; // the timers for refreshing data on window int plotRefreshRate $=1 ; / /$ the refresh rate (in $\mathrm{ms}$ ) for the RSSI plot

char port [2], rssin[7], rssi1[7], rssi2[7], rssi3[7], rssi4[7], rssi0[7]; /* strings int rssiPlot[pointsToPlot]; // the RSSI values stored in an array for the graph int PortNow, oldPort; // the current port number and the old port number before switc int switchingTime $=250 ; / /$ the switching time for each port double portsRSSI[numPorts]; // array to store the rssi samples when each port is swit int reScanSecs, reScanRoundNo;

int $\mathrm{Va}=21 ; / /$ Wiring $\mathrm{Pi}$ pin number for $\mathrm{Va}$ (BCM pin 5, Physical Pin 29)

int $\mathrm{Vb}=22$; //Wiring $\mathrm{Pi}$ pin number for $\mathrm{Vb}$ (BCM pin 6, Physical $\mathrm{Pin}$ 31)

GtkWidget *thrshldSlider; // the slider (scale) to select the RSSI threshold for re-s pthread_t rssiThread, portSwitchThread; // the thread to update rssi from wlan0

/* Surface to draw rssi plot */

static cairo_surface_t $*$ surface $=$ NULL;

void manualPort1() \{

manualOn = TRUE; / $*$ activate manual analyze mode to prevent auto analyse from overrid startTimer $=$ FALSE;

// now switch on the appropritate combination of Va \& Vb corresponding to port 1 digitalWrite(Va, LOW); // switch off GPIO pin for Va 


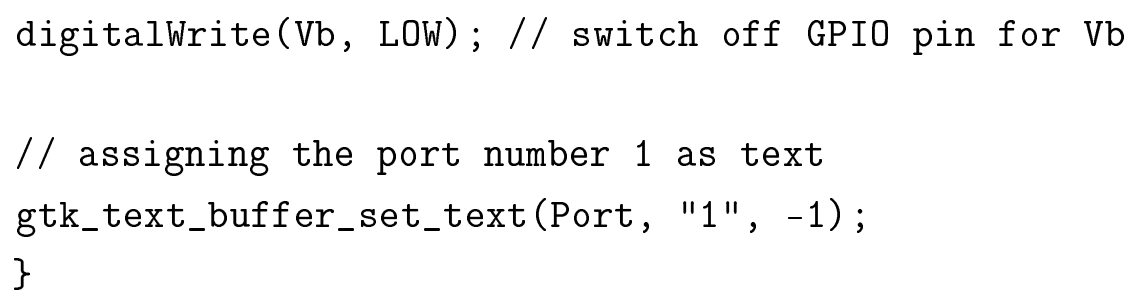

// now switch on the appropritate combination of Va \& Vb corresponding to port 4 digitalWrite(Va, HIGH); // switch off GPIO pin for Va 


\section{C code use for beam steering algorithm}

digitalWrite(Vb, HIGH); // switch on GPIO pin for Vb

// assigning the port number 4 as text

gtk_text_buffer_set_text(Port, "4", -1);

\}

void autoAnalyse() \{

switchNow = TRUE;

manualOn = FALSE;

startTimer = FALSE;

\}

void manualAnalyse() \{

switchNow = TRUE;

manualOn = TRUE;

startTimer = FALSE;

\}

void *portSwitch ()\{

int i;

double max;

do \{

if $($ switchNow $==1)\{$

/* scan all ports and find the port with lowest RSSI */

for $(i=0 ; i<$ numPorts; $i++)\{$

$/ /$ start switching

switch (i) \{

/* change the following lines for the appropriate GPIO pin number if numPorts $>4 * /$ case 0 :

gtk_text_buffer_set_text(Port, "1", -1);

delay(switchingTime / 2); // wait for duration indicated by variable switchingTime/2 digitalWrite(Va, LOW); // switch off GPIO pin for Va before scanning digitalWrite(Vb, LOW); // switch off GPIO pin for Vb before scanning delay(switchingTime / 2); // wait for duration indicated by variable switchingTime/2 
portsRSSI[0] = rssiNew; // store RSSI for the port in an array

sprintf(rssi1, $" \% .0 f \mathrm{dbm} "$, portsRSSI[0]);

gtk_text_buffer_set_text(P1RSSI, rssi1, -1);

break;

case 1:

gtk_text_buffer_set_text(Port, "2", -1);

delay (switchingTime / 2); // wait for duration indicated by variable switchingTime/

digitalWrite(Va, HIGH); // switch off GPIO pin for Va before scanning

digitalWrite(Vb, LOW); // switch off GPIO pin for Vb before scanning

delay (switchingTime / 2); // wait for duration indicated by variable switchingTime/

portsRSSI[1] = rssiNew; // store RSSI for the port in an array

sprintf(rssi2, "\%.Of dbm", portsRSSI[1]);

gtk_text_buffer_set_text(P2RSSI, rssi2, -1);

break;

case 2:

gtk_text_buffer_set_text(Port, "3", -1);

delay (switchingTime / 2); // wait for duration indicated by variable switchingTime/

digitalWrite(Va, LOW); // switch off GPIO pin for Va before scanning

digitalWrite(Vb, HIGH); // switch off GPIO pin for Vb before scanning

delay (switchingTime / 2); // wait for duration indicated by variable switchingTime/

portsRSSI[2] = rssiNew; // store RSSI for the port in an array

sprintf(rssi3, "\%.0f dbm", portsRSSI [2]);

gtk_text_buffer_set_text(P3RSSI, rssi3, -1);

break;

case 3:

gtk_text_buffer_set_text(Port, "4", -1);

delay (switchingTime / 2); // wait for duration indicated by variable switchingTime/ digitalWrite(Va, HIGH); // switch off GPIO pin for Va before scanning digitalWrite(Vb, HIGH); // switch off GPIO pin for Vb before scanning delay (switchingTime / 2); // wait for duration indicated by variable switchingTime/ portsRSSI[3] = rssiNew; // store RSSI for the port in an array

sprintf(rssi4, "\%.Of dbm", portsRSSI[3]);

gtk_text_buffer_set_text(P4RSSI, rssi4, -1);

break;

default:

break; 


\section{C code use for beam steering algorithm}

\}

\}

// find the port with the highest RSSI

$\max =-200$;

for $(i=0 ; i<$ numPorts; $i++)\{$

if (portsRSSI [i] $>\max )\{$

PortNow $=i ; / /$ set the port to switch to to the port with the highest RSSI $\max =$ portsRSSI[i]; // store the highest RSSI as a reference

\}

\}

/* switch on GPIO pin Va \& Vb corresponding to the selected port number */ switch (PortNow) \{

case 0 :

digitalWrite(Va, LOW); // switch off GPIO pin for Va before scanning digitalWrite(Vb, LOW); // switch off GPIO pin for Vb before scanning break;

case 1:

digitalWrite(Va, HIGH); // switch off GPIO pin for Va before scanning digitalWrite(Vb, LOW); // switch off GPIO pin for Vb before scanning break;

case 2:

digitalWrite(Va, LOW); // switch off GPIO pin for Va before scanning digitalWrite(Vb, HIGH); // switch off GPIO pin for Vb before scanning break;

case 3 :

digitalWrite(Va, HIGH); // switch off GPIO pin for Va before scanning digitalWrite(Vb, HIGH); // switch off GPIO pin for Vb before scanning break;

default:

break;

\}

// assigning the Selected port number as text sprintf(port, "\%d", PortNow +1 ); 


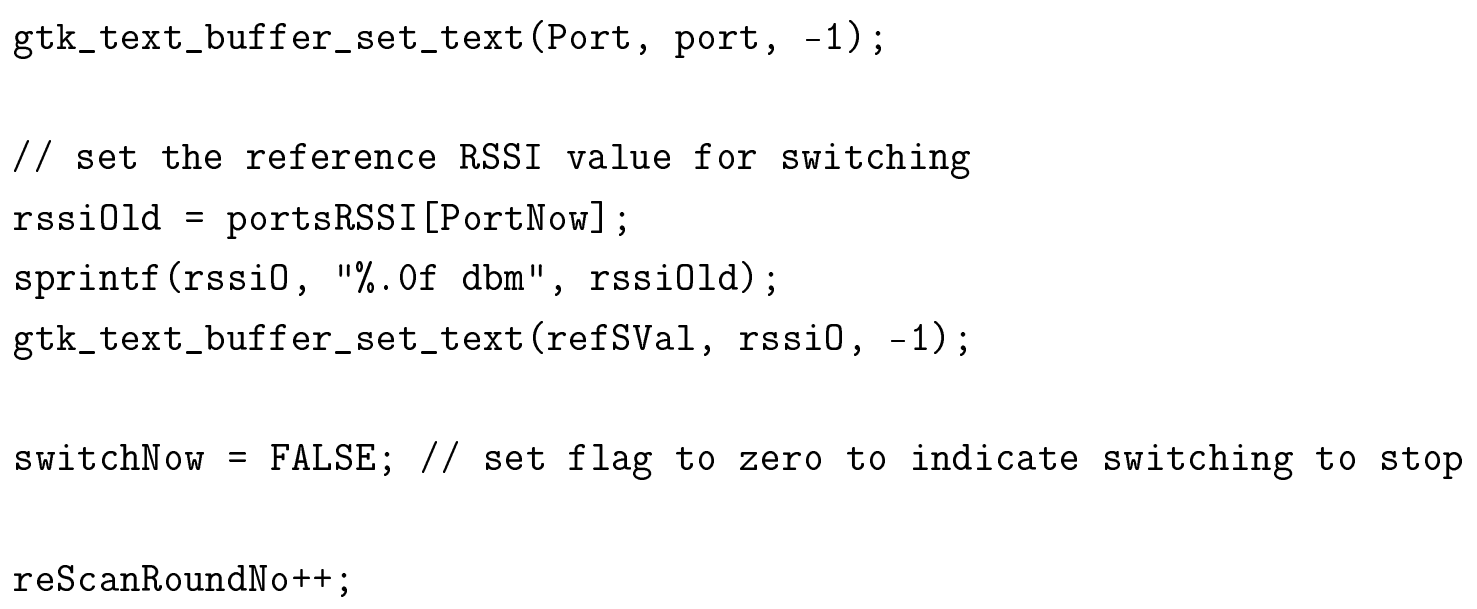




\section{C code use for beam steering algorithm}

int $j$;

do \{

// Execute a process listing

sprintf(command, "iwconfig wlanolgrep Signal|cut $-d \backslash " \wedge "-f 2 \mid c u t-d \backslash "=\backslash "-f 2 ")$;

pf $=$ popen (command, "r");

if $(! p f)\{$

fprintf(stderr, "Could not open pipe for output. $\backslash n$ ");

return NULL;

\}

else\{

// Grab data from process execution

fgets (data, 4, pf);

newRsireading $=$ atof $($ data) ;

if (newRssireading < 0$)\{/ /$ if rssi is in $\mathrm{dbm}$

rssiNew = newRssireading;

/* if new RSSI has dropped below the threshold then start scanning */

if $(($ rssiNew < gtk_range_get_value (GTK_RANGE $($ thrshldSlider $))$ ) \&\& (manualOn $==0)$ \&\& startTimer $=$ TRUE;

\}

else if ((rssiNew >= gtk_range_get_value (GTK_RANGE $($ thrshldSlider $))$ ) \&\& (manualOn $==0$ startTimer $=$ FALSE;

\}

// move data in array back by one slot

for $(j=$ (pointsToPlot -1$) ; j>0 ; j--)\{$

$\operatorname{rssiPlot}[j]=\operatorname{rssiPlot}[j-1]$;

\}

// copy new rssi value to beginning of array

rssiPlot $[0]=$ rssiNew;

\}

\}

if ( $\mathrm{pclose}(\mathrm{pf}) \quad !=0)$

fprintf(stderr, "Error: Failed to close command stream $\backslash n$ ");

\} while (!rssiStop); 


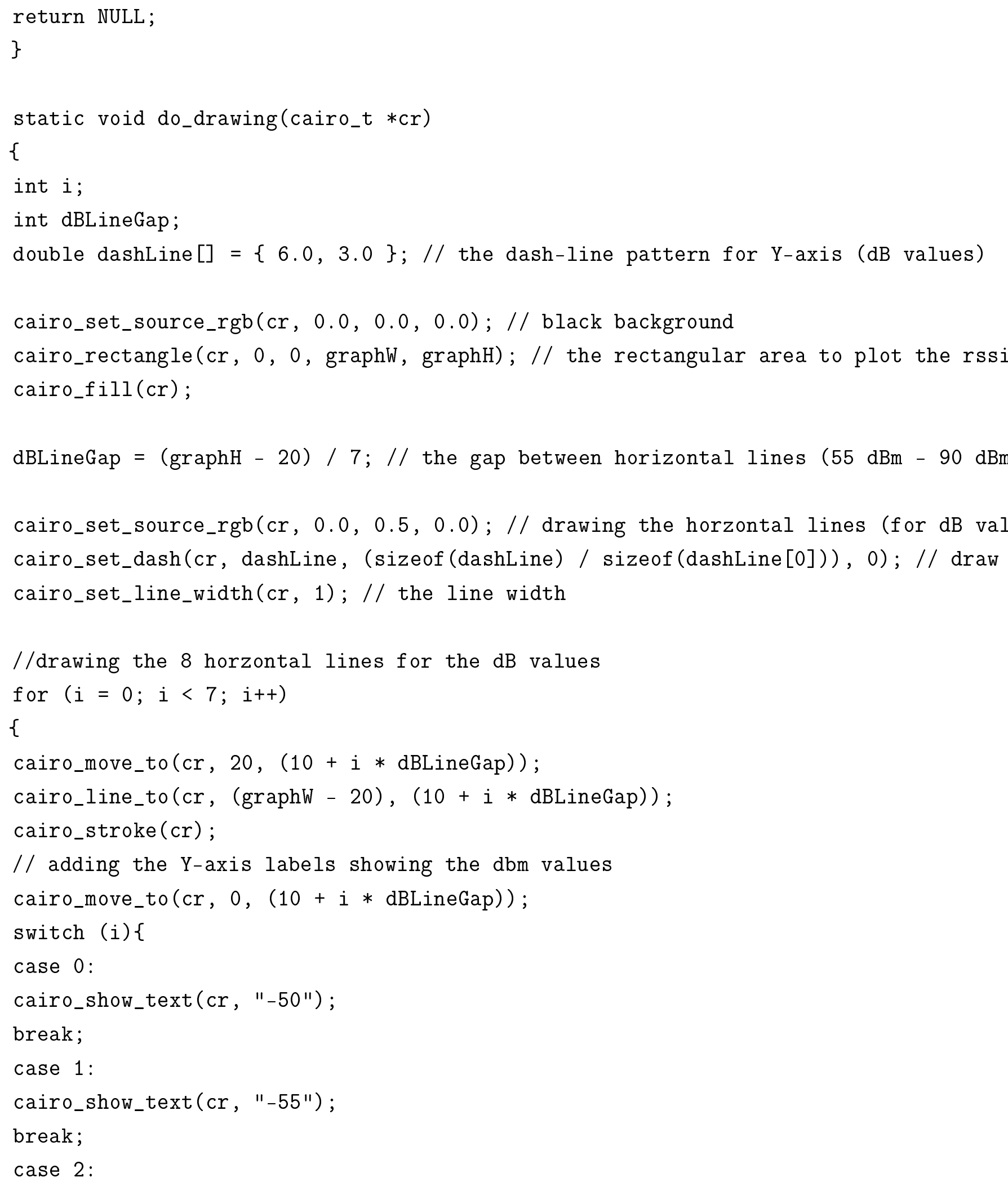




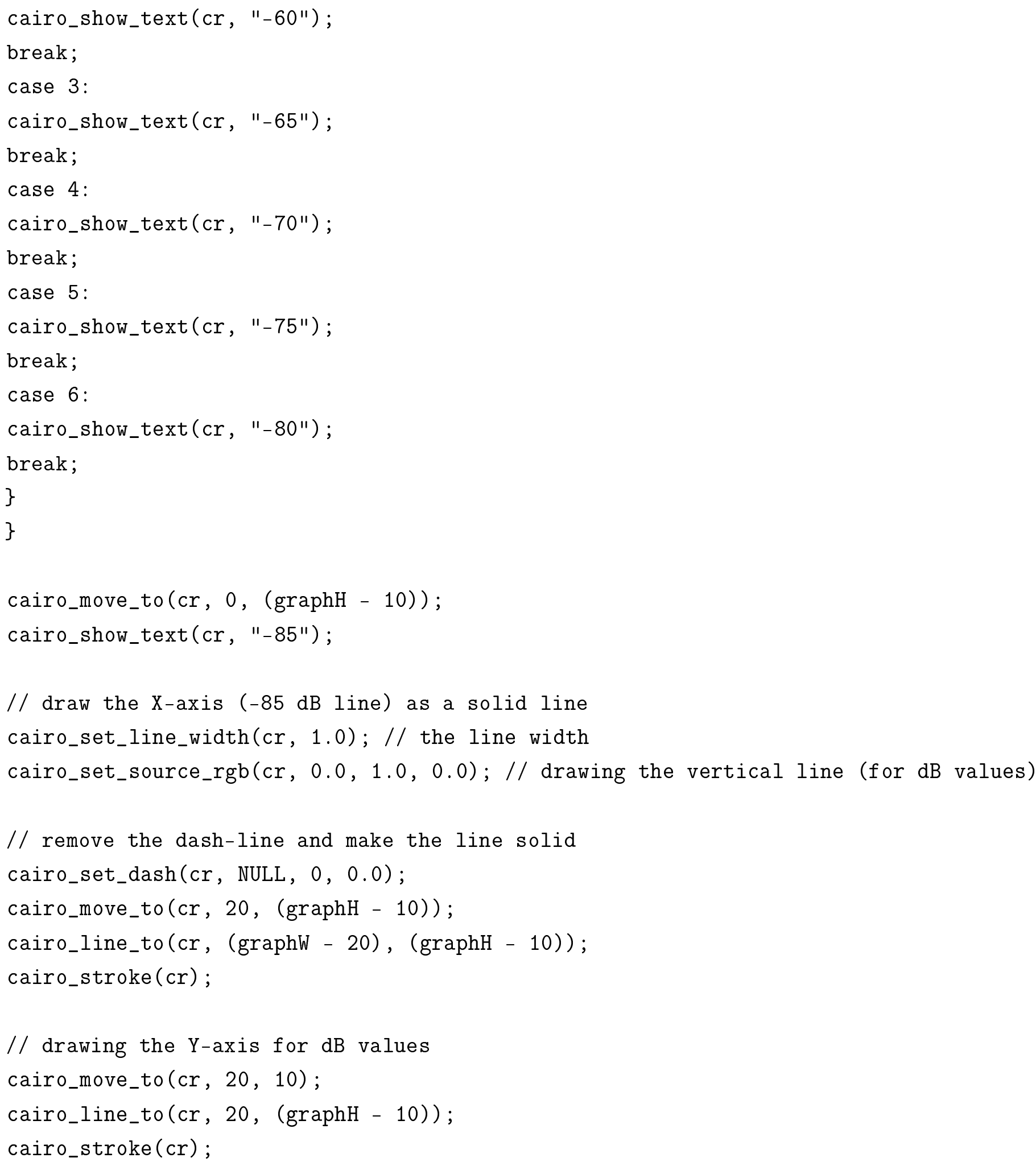


// drawing the real-time RSSI plot

for $(i=0 ; i<($ pointsToPlot -1$) ; i++)\{$

cairo_move_to $(\mathrm{cr},($ graphw $-20-(i * 10)),(10+(($ graphH -20$) / 35) *(-50-\operatorname{rssiF}$

cairo_line_to $(\mathrm{cr},(\mathrm{graph}-20-((i+1) * 10)),(10+((\mathrm{graphH}-20) / 35) *(-50$

cairo_stroke(cr);

\}

\}

static void clear_surface(void)

\{

cairo_t $*$ cr;

$c r=$ cairo_create $($ surface $) ;$

cairo_set_source_rgb(cr, 0, 0,0);

cairo_paint(cr);

cairo_destroy (cr);

\}

static gboolean on_draw_event(GtkWidget $*$ widget, cairo_t $* c r$, gpointer user_data)

\{

do_drawing $(c r)$;

return TRUE;

\}

static gboolean real_time_plot_draw(GtkWidget *widget, cairo_t $* c r$, gpointer user_c \{

clear_surface(); /* call function to clear the drawing surface used to plot the RSS

// draw the plot again again

gtk_widget_queue_draw(widget);

do_drawing $(c r)$; 
return TRUE;

\}

static gboolean updateRSSI()

\{

// converting real-time RSSI into a string with the term "dbm"

sprintf(rssiN, "\%.Of dbm", rssiNew);

// updating the RSSI and the progress bar

gtk_text_buffer_set_text(rssi, rssin, -1);

return TRUE;

\}

static updateRSCountdown(gpointer RSC)

\{

gchar *seconds;

if $($ startTimer $==1)\{$

if (reScanSecs $==0)\{$

switchNow = TRUE; // start re-scan when timer $=0$

seconds = g_strdup_printf("Re-scanning now");

\}

else\{

seconds = g_strdup_printf("Re-scan in $\% d "$, reScanSecs) $; / /$ convert seconds to str reScanSecs--;

\}

\}

else

\{

reScanSecs = 0 ;

reScanRoundNo = 0 ;

if (manualon $==1$ ) \{

seconds = g_strdup_printf("Manual Mode ON"); // No rescan when manual switching mode \}

else\{

seconds $=$ g_strdup_printf $(" R S S I>\% .0 f$ dBm", gtk_range_get_value(GTK_RANGE(thrshldSli 


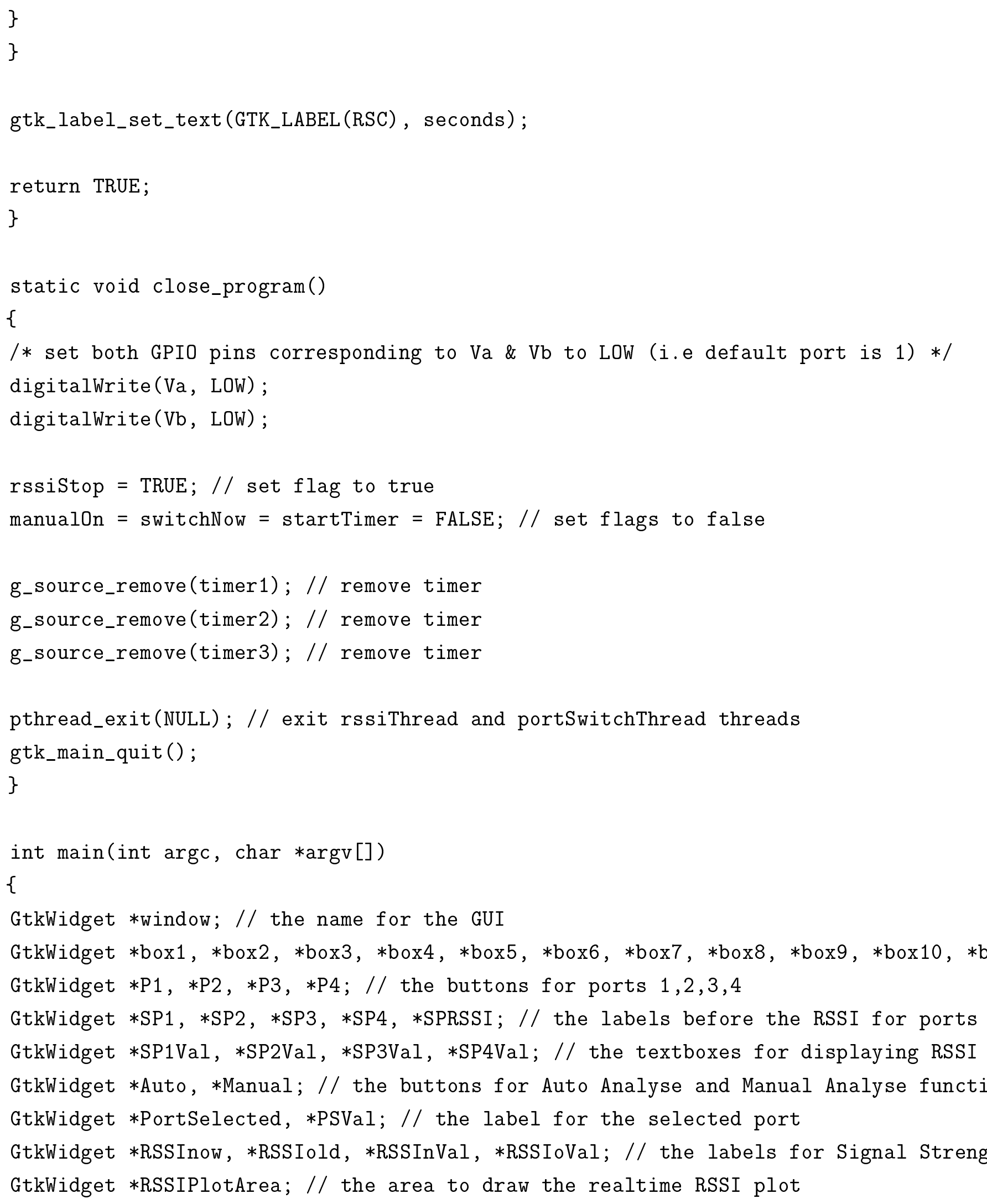


GtkWidget $*$ RSC; // the label for the countdown timer for periodic re-scan gtk_init(\&argc, \&argv);

rssiStop $=$ manualOn $=0 ; / /$ set flags to false

reScanSecs $=2$;

reScanRoundNo = 0 ;

gint RSSIValLen $=50 ; / /$ width of text boxes for displaying RSSI for ports $1,2,3,4$ gint TextBoxH $=15 ; / /$ height of text boxes for displaying RSSI for ports $1,2,3,4$

/* Initialising the GPIO pins and the flags for port switching */

wiringPiSetup(); // initialize the wiringPi library to use the GPIO pins

// The GPIO Pins to be used for sending voltage signals

pinMode(Va, OUTPUT); // setup wiringPi pin 21 (BCM pin 5/Physical pin 29) as an outpi pinMode (Vb, OUTPUT); // setup wiringPi pin 22 (BCM pin 6/Physical pin 31)as an outpit

//creating the window

window $=$ gtk_window_new(GTK_WINDOW_TOPLEVEL);

gtk_window_set_title(GTK_WINDOW(window), "SLA Beam Steering Application");

gtk_window_set_position(GTK_WINDOW (window), GTK_WIN_POS_CENTER);

// close the program upon closing the window

g_signal_connect(window, "destroy", G_CALLBACK(close_program), NULL);

// create the boxes to be put inside window

box1 = gtk_box_new(GTK_ORIENTATION_VERTICAL, 10);

box2 = gtk_box_new(GTK_ORIENTATION_HORIZONTAL, 10);

box3 = gtk_box_new(GTK_ORIENTATION_VERTICAL, 10);

box4 = gtk_box_new(GTK_ORIENTATION_VERTICAL, 10);

box5 = gtk_box_new(GTK_ORIENTATION_HORIZONTAL, 10);

box6 = gtk_box_new (GTK_ORIENTATION_HORIZONTAL, 10);

box7 = gtk_box_new(GTK_ORIENTATION_VERTICAL, 10);

box8 = gtk_box_new(GTK_ORIENTATION_HORIZONTAL, 10);

box9 = gtk_box_new(GTK_ORIENTATION_HORIZONTAL, 10);

box10 = gtk_box_new(GTK_ORIENTATION_VERTICAL, 10); 


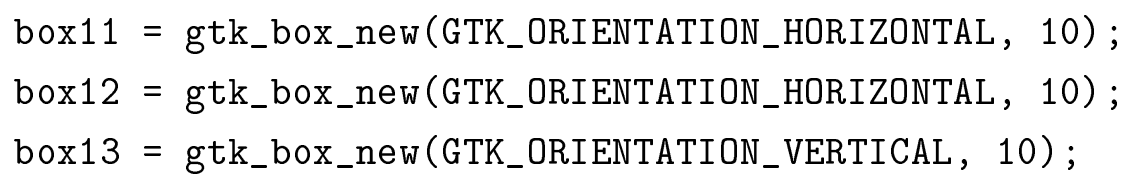

// adding the labels for displaying RSSI levels for ports 1,2,3,4

SPRSSI = gtk_label_new("RSSI during scan"); // The text field "RSSI" on top $\mathrm{SP} 1$ = gtk_label_new ("Port 1");

$\mathrm{SP} 2$ = gtk_label_new("Port 2");

$\mathrm{SP} 3=$ gtk_label_new ("Port $3 ")$;

SP4 = gtk_label_new("Port 4");

// defining the size of those labels

gtk_widget_set_size_request(SPRSSI, SPw, SPh);

gtk_widget_set_size_request (SP1, SPw, SPh);

gtk_widget_set_size_request (SP2, SPw, SPh);

gtk_widget_set_size_request (SP3, SPw, SPh);

gtk_widget_set_size_request (SP4, SPw, SPh);

// defining the textboxes for RSSI values

SP1Val = gtk_text_view_new();

SP2Val = gtk_text_view_new();

SP3Val $=$ gtk_text_view_new();

SP4Val = gtk_text_view_new();

$/ /$ set text boxes to non-editable and hide the cursor

gtk_text_view_set_editable(GTK_TEXT_VIEW(SP1Val), FALSE);

gtk_text_view_set_editable(GTK_TEXT_VIEW(SP2Val), FALSE);

gtk_text_view_set_editable(GTK_TEXT_VIEW(SP3Val), FALSE);

gtk_text_view_set_editable(GTK_TEXT_VIEW(SP4Val), FALSE);

gtk_text_view_set_cursor_visible(GTK_TEXT_VIEW(SP1Val), FALSE);

gtk_text_view_set_cursor_visible(GTK_TEXT_VIEW(SP2Val), FALSE);

gtk_text_view_set_cursor_visible(GTK_TEXT_VIEW(SP3Val), FALSE);

gtk_text_view_set_cursor_visible(GTK_TEXT_VIEW(SP4Val), FALSE);

// defining the text buffers to hold the RSSI values for text boxes

P1RSSI = gtk_text_view_get_buffer (GTK_TEXT_VIEW(SP1Val));

P2RSSI = gtk_text_view_get_buffer (GTK_TEXT_VIEW(SP2Val));

P3RSSI = gtk_text_view_get_buffer (GTK_TEXT_VIEW(SP3Val));

P4RSSI = gtk_text_view_get_buffer $($ GTK_TEXT_VIEW (SP4Val)); 
// defining the size of the text boxes gtk_widget_set_size_request(SP1Val, RSSIValLen, TextBoxH); gtk_widget_set_size_request(SP2Val, RSSIValLen, TextBoxH); gtk_widget_set_size_request(SP3Val, RSSIValLen, TextBoxH); gtk_widget_set_size_request(SP4Val, RSSIValLen, TextBoxH);

// defining the positioning of those labels gtk_box_pack_start(GTK_BOX(box1), SPRSSI, FALSE, TRUE, 0); gtk_box_pack_start(GTK_BOX(box3), SP1, FALSE, TRUE, 0); gtk_box_pack_start(GTK_BOX (box3), SP2, FALSE, TRUE, 0); gtk_box_pack_start(GTK_BOX(box3), SP3, FALSE, TRUE, 0); gtk_box_pack_start(GTK_BOX(box3), SP4, FALSE, TRUE, 0);

gtk_box_pack_start(GTK_BOX (box4), SP1Val, FALSE, TRUE, 0); gtk_box_pack_start(GTK_BOX(box4), SP2Val, FALSE, TRUE, 0); gtk_box_pack_start(GTK_BOX (box4), SP3Val, FALSE, TRUE, 0); gtk_box_pack_start(GTK_BOX (box4), SP4Val, FALSE, TRUE, 0);

gtk_box_pack_start(GTK_BOX(box2), box3, FALSE, TRUE, 10); gtk_box_pack_start(GTK_BOX(box2), box4, FALSE, TRUE, 0);

gtk_box_pack_start(GTK_BOX(box1), box2, FALSE, TRUE, 0);

// adding the buttons for manual switching for ports 1,2,3,4 P1 = gtk_button_new_with_label("1");

P2 = gtk_button_new_with_label("2");

P3 = gtk_button_new_with_label("3");

P4 = gtk_button_new_with_label("4");

// defining the size of those buttons gtk_widget_set_size_request ( $\mathrm{P} 1, \mathrm{Pw}, \mathrm{Ph})$; gtk_widget_set_size_request (P2, $\mathrm{Pw}, \mathrm{Ph})$; gtk_widget_set_size_request (P3, Pw, Ph); gtk_widget_set_size_request( $\mathrm{P} 4, \mathrm{Pw}, \mathrm{Ph})$;

// the functions to call when the buttons are pressed g_signal_connect(G_OBJECT (P1), "clicked", G_CALLBACK (manualPort1), NULL); g_signal_connect(G_OBJECT (P2), "clicked", G_CALLBACK (manualPort2), NULL); 
g_signal_connect (G_OBJECT (P3), "clicked", G_CALLBACK (manualPort3), NULL);

g_signal_connect(G_OBJECT (P4), "clicked", G_CALLBACK (manualPort4), NULL);

// defining the positioning of those buttons

gtk_box_pack_start(GTK_BOX (box5), P1, FALSE, TRUE, 0);

gtk_box_pack_start(GTK_BOX (box5), P2, FALSE, TRUE, 0);

gtk_box_pack_start(GTK_BOX(box6), P3, FALSE, TRUE, 0);

gtk_box_pack_start(GTK_BOX (box6), P4, FALSE, TRUE, 0);

gtk_box_pack_start(GTK_BOX(box1), box5, FALSE, TRUE, 0);

gtk_box_pack_start(GTK_BOX(box1), box6, FALSE, TRUE, 0);

// To show the real-time RSSI

RSSInow = gtk_label_new("RSSI now: ");

// defining the textbox to display the RSSI

RSSInVal $=$ gtk_text_view_new ();

// defining the size of the textbox

gtk_widget_set_size_request(RSSInVal, RSSIValLen, TextBoxH);

// set text box to non-editable and hide the cursor

gtk_text_view_set_editable(GTK_TEXT_VIEW(RSSInVal), FALSE) ;

gtk_text_view_set_cursor_visible(GTK_TEXT_VIEW(RSSInVal), FALSE);

// defining the text buffer to hold the port number

rssi $=$ gtk_text_view_get_buffer(GTK_TEXT_VIEW(RSSInVal));

// defining the position of the textbox

gtk_box_pack_start(GTK_BOX(box9), RSSInow, FALSE, TRUE, 0);

gtk_box_pack_start(GTK_BOX (box9), RSSInVal, FALSE, TRUE, 0);

// The Re-scan threshold RSSI value label

RSSIold = gtk_label_new("Re-scan Threshold (dBm): ");

// the slider (scale) to select the RSSI threshold

thrshldSlider = gtk_scale_new_with_range(GTK_ORIENTATION_HORIZONTAL, sliderRangeMin gtk_range_set_value (GTK_RANGE(thrshldSlider), -30);

gtk_box_pack_start(GTK_BOX (box10), RSSIold, FALSE, FALSE, 0);

gtk_box_pack_start(GTK_BOX(box10), thrshldSlider, FALSE, FALSE, 0);

// The 'Auto Analayse' and 'Manual Analyse' buttons 
Auto = gtk_button_new_with_label("Auto Analyse");

Manual = gtk_button_new_with_label("Manual Analyse");

// the functions to call when these buttons are pressed

g_signal_connect(G_OBJECT (Auto), "clicked", G_CALLBACK (autoAnalyse), NULL);

g_signal_connect (G_OBJECT (Manual), "clicked", G_CALLBACK (manualAnalyse), NULL);

// defining the positioning of those buttons

gtk_box_pack_start(GTK_BOX(box11), Auto, FALSE, TRUE, 0);

gtk_box_pack_start(GTK_BOX(box11), Manual, FALSE, TRUE, 0);

// displaying the text for selected port number

PortSelected = gtk_label_new ("Port selected: ");

// defining the textboxes for selected port number

PSVal $=$ gtk_text_view_new () ;

// set text box to non-editable and hide the cursor

gtk_text_view_set_editable(GTK_TEXT_VIEW(PSVal), FALSE);

gtk_text_view_set_cursor_visible(GTK_TEXT_VIEW(PSVal), FALSE);

$/ /$ defining the text buffer to hold the port number

Port $=$ gtk_text_view_get_buffer $($ GTK_TEXT_VIEW(PSVal));

$/ /$ assigning the Selected port number as text

sprintf(port, "\%d", PortNow);

gtk_text_buffer_set_text(Port, port, -1);

// defining the size and position of the textbox and label

gtk_widget_set_size_request(PSVal, (RSSIValLen / 3), TextBoxH);

gtk_box_pack_start(GTK_BOX(box12), PortSelected, FALSE, TRUE, 0);

gtk_box_pack_start(GTK_BOX(box12), PSVal, FALSE, TRUE, 0);

// defining text label for the periodic scan's countdown timer

RSC = gtk_label_new ("Autoscan mode ON");

gtk_widget_set_size_request (RSC, $(2.5 * \mathrm{Pw}), \mathrm{Ph})$;

gtk_box_pack_start(GTK_BOX(box7), box9, FALSE, TRUE, 0);

gtk_box_pack_start(GTK_BOX (box7), box10, FALSE, TRUE, 0);

gtk_box_pack_start(GTK_BOX (box7), box11, FALSE, TRUE, 20);

gtk_box_pack_start(GTK_BOX (box7), box12, FALSE, TRUE, 0);

gtk_box_pack_start(GTK_BOX(box7), RSC, FALSE, TRUE, 0); 
// the area for drawing the RSSi plot

RSSIPlotArea $=$ gtk_drawing_area_new () ;

gtk_widget_set_size_request(RSSIPlotArea, graphW, graphH) ;

gtk_box_pack_start(GTK_BOX(box13), RSSIPlotArea, FALSE, TRUE, 50);

g_signal_connect (G_OBJECT (RSSIPlotArea), "draw", G_CALLBACK (on_draw_event), NULL);

gtk_box_pack_start(GTK_BOX (box8), box1, FALSE, TRUE, 20);

gtk_box_pack_start(GTK_BOX (box8), box7, FALSE, TRUE, 20);

gtk_box_pack_start(GTK_BOX(box8), box13, FALSE, TRUE, 20);

gtk_container_add(GTK_CONTAINER(window), box8);

// new threads for reading RSSI and updating graph

pthread_create(\&portSwitchThread, NULL, \&portSwitch, NULL);

pthread_create(\&rssiThread, NULL, \&rssiRead, NULL);

gtk_widget_show_all(window);

SwitchNow = TRUE;

timer1 = g_timeout_add (plotRefreshRate, updateRSSI, NULL);

timer2 = g_timeout_add (plotRefreshRate, (GSourceFunc)real_time_plot_draw, (gpointe

timer3 = g_timeout_add (1000, (GSourceFunc) updateRSCountdown, RSC);

gtk_main ();

return 0 ;

\} 
